



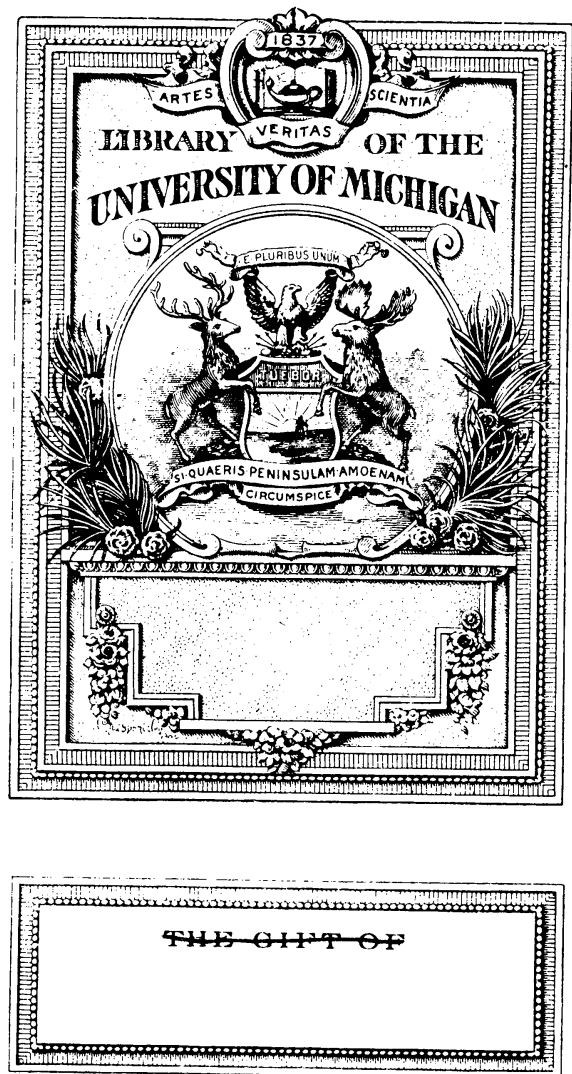
Chemical Libreny

$Q \square$

443

. 98 
at

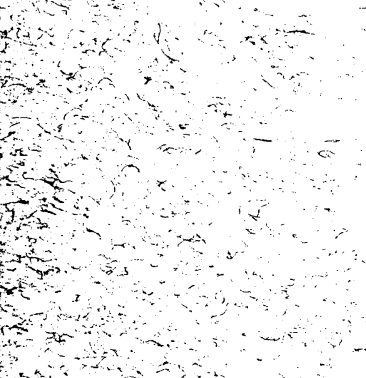

$x$ 


\section{ON THE WATER RELATIONS OF THE COCONUT PALM (COCOS NUCIFERA) - ON THE OIL PRODUUCED FROM THE NUTS-THE FACTORS ENTERING INTO THE RANCIDITY OF THE OIL, AND THE INSECTS ATTACKING THE TREES.}

Introduction by Paul C. Freer.

Investigations on the subject of the coconut palm (Cocos nucifera) have been carried on in the Bureau of Government Laboratories for the past eighteen months. The work has been divided into three parts and brought to its present state by coöperation between several divisions of the institution. It will be published in serial form in the Journat. The first portion covers the water relations of the tree from the standpoint of its physiology, by Dr. Edwin Bingham Copeland, who spent several months on a plantation studying this question from an experimental standpoint. The second paper covers the coconut in its relation to the cultivation of the tree and the production of coconut oil, and includes a study of the deterioration both of the copra and the oil by reason of rancidity caused by molds and bacterial growth, by Herbert S. Walker; and in conclusion there is added a study of the insects which attack the plant, together with suggestions as to the best means of combating their depredations, by Charles S. Banks and William Schultze.

By this union of the laboratory work, the study of this most important tropical tree has been carricd to an extent which not only will enable the conclusions to be of great value to planters but which will also have a scientific interest for those who are not immediately interested in coconut production. One topic which is of especial importance is still under investigation and not ready for publication. This is the study of the germinating nut together with the transformation which the oil undergoes during the growth of the embryo. This topic offers an opportunity for the study of the enzymes in a germinating plant which is unsurpassed, as the size of the seed of the coconut and the ease with which it is separated into its various constituent parts brings a certainty of results not to be encountered in other instances. This portion of the investigation is now being followed in the chemical laboratory. When the serial on the subjects mentioned above has been completed it will be published as a separate reprint. 
San Ramon Government Farm, where most of these investigations were carried on, lies on the west coast of Mindanao 10 miles north of the town of Zamboanga. It extends for about 2 miles along the seacoast and toward the interior for 3 or 4 miles, to the base of a small range of densely wooded mountains, which forms an admirable watershed.

Four small streams run through San Ramon from the mountains to the sea. It is very probable that there is considerable underground drainage as well, for fresh water may be obtained at a depth of 5 or 6 feet almost anywhere along the shore, even at the elge of the beach. At present copra and hemp are the principal products of the farm, together with a little cacao.

At the time of writing all the coconut trees used for making copra at San Ramon were planted by the Spanish, but large numbers of new ones are being set every year from selected seed, for which only the largest and best nuts are taken. They are laid out on the ground in a sheltered place and a small section of husk is cut from the top of each to afford a more easy egress for the sprout. At the end of about six months' time, when the sprout is from 2 to 3 feet high and the nut has just begun to take root in the ground, it is ready for planting. For this purpose a hole about 2 feet deep is prepared and the young plant is firmly packed with the soil, so that the sprout stands erect and the top of the nut is 6 to 10 inches below the surface. As a protection against wild hogs it has of late been the custom to dig a pit 4 or 5 feet deep and to plant the nuts at the bottom of this. The seedlings are set out in straight rows, allowing a space of about 10 meters between each plant.

After planting, the young coconut requires very little care, except to keep it free from weeds and the attacks of animals and insects, until it reaches maturity. The average time before a tree begins to give a good yield of fruit may be set at ten years. Instances have been known when bearing commenced as early as the fifth year, but these are of rare occurrences and under exceptionally favorable circumstances.

The process in use for preparing copra is very simple. The nuts are gathered by natives, who climb the trees, cut off the ripe or nearly ripe fruit, and let it fall to the ground. No especial care is taken to prevent damage by falling. The nuts are then piled in a heap and allowed to stand for a few weeks before being opened. To remove the outer, fibrous husk the natives make use of a heavy spearhead firmly sunk in the ground. They force the nut down on the sharp point until it penetrates to the shell, then, by a peculiar twist, strip off the husk, a portion at a time. One man can husk, on an average, 1,000 nuts per day.

After being thus prepared the coconuts are split in halves by a couple of sharp blows from the back of a bolo. 'The milk is allowed to go to waste on the ground.

Drying.-The simplest method of drying the meat is to spread out the halves of the coconut on large wooden trays, face up, in the sun. At 
night and in case of rain the trays are piled under a shed. After standing in the sun for two or three days the meat becomes partially dry and has shrunken sufficiently to permit its removal from the shell. It is then put back on the trays and again exposed for a few days until it is thoroughly desiceated.

The other method of preparing copra in use at San Ramon is to pile the coconut halves, face downward, on a bamboo grating over a slow fire of husks which is burning in a thick-walled brick kiln about 6 feet high, the whole being inclosed in a large shed. By this arrangement it is sufficient to dry the nuts over night before removing the shells.

$\Lambda$ fter heating the meat in the same manner during four or five hours on the next day, it is ready to store for the market. "Grill-dried" copra prepared in this way is not quite so liable to be attacked by insects and molds, but on account of its dark color and slightly smoky flavor it is considered inferior in quality to the sun-dried article. 


\title{
ON THE WATER RELATIONS OF THE COCONUT PALM (COCOS NUCIFERA).
}

\author{
By Edwin Bingham Copeland.
}

(From the Botanical Section of the Biological Laboratory, Bureau of Science.)

The work on Cocos nucifera (coconut), the result of which is reported below, was performed at the Government farm at San Ramon, near Zamboanga. Its purpose was to acquire as thorough a knowledge of the physiology of this palm as the field conditions would permit, with the especial hope that the results would be available for improving existing methods of the plant's cultivation.

Because of the remoteness of the place of work from any library or base of supplies, the simplicity of apparatus which for the greater part is used in investigating all phases of a plant's transpiration, the writer's familiarity with this particular field, obtained in the preparation of earlier papers, and because of the very great practical importance of understanding this phase of the physiology of any plant important in agriculture, the work was principally focused on the water relations of the coconut. At the same time other phases of the tree's activity were not neglected; and, in cases where it seemed worth while, notes not bearing on the main subject are included in this paper. The value of artificial or natural fertilizers was not considered, because this question is more in the domain of the agriculturalist.

The divisions of the main subject are treated in the following order: The root-its structure and growth, and the absorption of water; the leaf-its structure, the activity of the stomata, and the transpiration; with final conclusions as to the fitness of the plant for its characteristic habitat and suggestions as to its most advantageous cultivation.

\section{THE ROOT.}

The roots of Cocos nucifera have the two typical root functions-the anchoring of the tree and the absorption of the water and mineral food necessary for its maintenance and growth. In the absence of a taproot, or of any great roots the hold of which in the ground can maintain the rigidity of the trunk, the mechanical problem of the firm anchorage of the latter finds a solution essentially different from that which we are accustomed to encounter in the casc of dicotyledonous trees. The base of 
the trunk is convex or obconical, and is usually buried for a depth of hardly more than jo entimeters. Its surface underground is almost entirely covered with the bases of the roots. 'The latter are remarkably milorm, about 1 aentimeter in diameter, radiating from the tree on all sides, each without much variation in its direction, and, so far as my observations justify a general conclusion, for a normal distance of about 5 meters in firm soil and 7 meters in sand. 'The lateral branches, whatever direction they may take with regard to the action of gravity, leave the main roots with surprisingly uniform exactness at right angles and are likewise on the whole straight, though less so in detail than the main roots.

'The old main roots are notable for the combination of elasticity ${ }^{1}$ and tensile strength shown by their powerful central steles, the cylinder of xylem inclosing a "pith" with thick, lignified walls. 'The most conspicuous feature of the branches is their stiffness, for which the stele is not more responsible than the hypodermis. I have never before, in any plant, seen a rigidity on the part of the fine, absorbing roots which will compare with that possessed by those of the coconut. The intimate contact between the hard, firm roots and the soil is responsible for the rigidity of the Cocos, as of other trees, but while in most, this contact is centered about the base of the trunk, the Cocos has it disseminated equally through the ground to a radius of 5 meters or more. The main roots act as so many taut strands between the base of the trunk and the multitude of fine points of attachment. 'The effectiveness of the coconut's system of anchorage is perfect. 'The tree's favorite habitat is the seashore, where it receives the unbroken force of the fiercest storms. Because of its elasticity, the trunk very rarely breaks, and I have never seen one instance of an uprooted coconut, the roots of which had not either previously been killed or undermined by waves.

Eighty centimeters is not a very exceptional diameter for a well-grown bole, though a majority fall below this size. The buried part of a stem of this thickness will afford attachment for nearly 8,000 bases of roots 1 centimeter in diameter. Some of the main roots bear few or no branches at all like themselves; others have 10 to 20 , which rarely reach a length of 1 meter or a diameter of 4 millimeters. The main roots and these major branches bear numerous fine ones, 1 to 2 millimeters in diameter, springing forth at right angles and having a rigidity which has already been noted. These may be the ultimate divisions; or they in turn may bear finer branches, at most a very few centimeters long, about 0.5 millimeter in diameter; the life of the latter is transitory like that of root hairs. A. less ample system of branches is formed in sand than in firmer ground.

Dead, distal parts of roots are replaced from the bases of the same roots

${ }^{1}$ Pfeffer, Pflanzenphysiologie, II, page 60, cites Sonntag, Landw. Jahrb. (1892), 21, 839 as authority for a stretching of 20 per cent by Cocos fibers, without exceeding their limit of elasticity. 
in such a way that the new takes the place of the old, not only as an alsorling organ but in the mechanical system as well. At the point where dying back ceases, a root, or frequently two roots, spring from the end of the part which is still living. 'Their origin apparently is internal, and, as is to be expected, from the outer limit of the stele; the hypodermis of these older parts of old roots is so strong that the young ones are rarely able to break through it, with the result that they grow onward within the shell, sometimes for 30 centimeters or more, before the hypodermis is sufficiently decomposed to permit their escape. The direction of growth is then well fixed. From an observation of exceptional cases in which the young root succeeded in rupturing the hypodermis at its origin, and in which it then grew along or near it, it appears that as a phenomenon of "correlation" the young root has the same orientation-reaction as the one it replaces. 'The old hypodermal shell is a most effective aid in this reaction.

My observations on the rapidity of the growth of roots have been unsatisfactory. Many times I have marked off zones on apparently healthy roots only to discover that they showed no subsequent growth. Some, for a time, have elongated little or not at all, then for a few days have grown vigorously, then stopped, without any apparent reason for the irregularity :

The most rapid growth I have measured was 3.5 millimeters per diem. In a month three roots grew more than 4 centimeters, but none as much as 5 centimeters. Sometimes, under favorable conditions, there may be a much more rapid growth than I have been able to observe; 3.5 millimeters per diem is hardly more than 1 meter per annum, a rate too slow to be accepted without more evidence. A part of the roots I examined grew in water and a part in air surrounded by soil. Those which elongated considerably in water at the same time became more slender.

In large and rapidly growing roots a little elongation occurs in a zone 10 to 15 millimeters from the tip (not from the growing point), but in most cases it is confined to the apical 10 millimeters. The root whose growth was most rapid was 9 millimeters in diameter and had a cap 10 to 11 millimeters long. In two days the latter grew 0.5 millimeter, 5 millimeters of root grew out of it, and the zone immediately outside grew 1.5 millimeters. The length of the cap is somewhat greater than the diameter of the root, which is usually about equal to the length of the growing zone when measured from the outside tip; therefore all growth is generally within the cap. In this case the cap grew one-tenth as rapidly as the root, and this seems to be about the usual ratio. In the ground the resistance to the passage of the moving tip results in a continual tearing off of the outer layers of the cap, these layers usually persist in the form of collars around the root, and each is about as long as the cap; altogether they not infrequently form a sheath along the whole younger part of the root. It is possible that these collars or sheaths facilitate the absorption of water. When the root grows without friction, in water, the whole outer portion of the cap, while retaining its form, is occasionally sloughed off.

No response to any other directive agent is so conspicuous as the autotropism of the coconut roots, of whatever order. The general level of the main roots is maintained by a combination of hydrotropism and aërotropism, which I have not 
lwen able to analyze. A reoent paper by bemett" shows that many cases, at. least, of apparent aibotropisill alre really hydrotropie, and the same is probubly true with the cocos. The roots maintain a derper level in sand than in heavy soil. When the other stimuli are removed, a variable geotropism shows itself; some, in water, grow straight ahead in as nearly horizontal a direction as it was convenient to arrange them in the bottle; but the majority show a feeble positive geotropism, the most rapidly executed curve being $40^{\circ}$ in two days. The secondary roots are usually controlled by their autotropism alone. In heavy soil they are sometimes more numerous on the upper sides of the main roots, probably because of an induced geoanxesis, since the structure of the roots precludes the pobability of any direct locative influence of moisture on their origin, and the pnemmathode roots appear on all sides. In nature, no roots will grow to any distance into water, nor into a level of the soil where water stancls; and a rise in the water level ultimately kills the submerged ones.

Root structures.-'The stelar tissues of the coconut root offer very little that needs description. The number of xylem rays is usually 40 or more in the larger, 10 to 15 in the branch roots, 1 to 1.5 millimeters in diameter, and fewer in the finer ones. In the young parts of the main ones the pith is parenchymatous, with very thin walls. The latter begin to thicken at a distance from the apex at which both hypodermis and endodermis have reached their permanent state. They then become very thick throughout, and are the chief source of the root's great tensile strength.

In cross sections, a very few cells behind the growing point, the pericycle is distinguishable by the regularity and the large size of its cells. 'The latter eventually become somewhat flattened tangentially, but they still form a conspicuous layer in sections of old roots, as their walls remain thin and colorless. The cross partitions are reticulate-punctured.

In very young parts of the root the endodermis can be identified only by reference to the pericycle (fig. 2). Its cells begin to thicken at about the same point as do those of the hypodermis, where the latter begins to interfere with the absorption of water. The thickening takes place cell by cell, rather abruptly in the individual cells, but without any uniformity throughout the layer, so that in some sections a few will be found well thickened, all the others still thin; while a little farther back most of them will be found to be thick. Counting all the endodermal cells in a section, an undue proportion of those which thicken late is directly outside the xylem rays, where passage cells would be expected. However, cells in this position are not infrequently among the first to thicken, whereas scattered ones found elsewhere are often among the last. Considering the zone with reference to the hypodermis at which the thickening of the endodermis begins, it is evident that it is only as the water travels obliquely up the root to the stele, and not directly inward, that any of the cells remaining thin have occasion to serve in its passage. The appearance of the old endodermis is shown in the accompanying figure (fig. 1).

${ }^{2}$ Bot. Gaz. (1904), 37, 241. 
'The outer part of the cortex, immediately underlying the epidermis to a depth of three to six layers, is composed of cells smaller than the deeper-lying ones. 'I'he walls of these, while they are in young and antive parts of the roots, are very thin, with a notably dense protoplasm. Subsequently the walls thicken, those of the outermost cells first, until the lumen is almost obliterated; they acquire a stony hardness and dark color (figs. 3 and 4), thus forming a closed shell around the root, protecting it against animals or fungi and having a mechanical value already mentioned. The imperviousness of this shell to water is shown by its effect on the epidermis and on the formation of pneumathodes. 'I'he zone in which the hypodermis forms is that at which the root ceases to absorb water from the soil.

The larger the root, the farther from the tip is this likely to be. In very active ones the distance is as much as 5 centimeters; in those less active, but by no means inert, having a diameter of 7.5 millimeters, it is found to be 2 centimeters from the tip, while during drought it advances to a position well within the firmly adherent part of the cap.

Between the hypodermis and endodermis the cortex is composed of rather large cells, isodiametric or somewhat elongate longitudinally, with thin, colorless walls, watery contents, and numerous intercellular spaces (fig. 5). After the layers bounding it reach their final state, parts of the interlying cortex become unequally thick walled and lignified. At the basal end of old roots this internediate cortex breaks, probably as a result of tension between the elastic stele and nonelastic shell, leaving the former loose inside of the latter.

The dermatogen is questionably distinguishable around the growing point, even in most favorable sections. The epidermis is a transitory tissue, dying when its connection with the inner part of the root is interrupted by the development of the hypodermis. Its most conspicuous feature is that the least diameter of its cells is the longitudinal (figs. 6-8). No root hairs are ever formed, but the superficial area is slightly increased by the breaking apart of the outer ends of the cells-a process which is most evident in longitudinal sections. In a soil where the supply of water is even moderately constant and ample the coconut root, with its short absorbing zone and absence of hairs, would be regarded as but a poor water gatherer, but when water is abundant, hairs are not needed; and in a dry time their sacrifice is spared to the coconut. A tree whose normal economy is planned on the absence of root hairs is comparatively well able to survive periods of abnormal difficulty in obtaining water.

Pneumathodes (figs. 9-14).-The development of the hypodermal shell so completely cuts off the interior of the root from all communication with the outside that it can not carry on the limited exchange of gases necessary for its respiration, and therefore it is obliged to develop special breathing organs, or "pneumathodes." These are specialized roots which quickly grow to a length of from 3 to 6 millimeters and then abruptly 
stop. 'The cells of the cortex then enlarge; at first they keep their form, but alterwards they become spherical and finally put out processes each of which keeps in contact with a corresponding one from an adjacent coll. 'I'his enlargement of the cortex ruptures the epidermis and the growth of the inner layers separates the outer ones, so that the epidermis and outer layers flare back from both ends of the swollen zone; its surface is then mealy in appearance and white because of the contained air. As the pneumathode ages, the cap and all the outer tissues beyond the open zone slough off ; the strongly lignified stele gives it stability and its sharp point will protect it against mechanical injury, if protection is needed. 'The cells of the open tissue neeessarily promptly die, but their walls remain firm, their surfaces become granular, and in this eondition they can not be wetted, so that the large amount of air contained between them can not be displaced. The cells next to the stele, and those at the base of the pneumathode-that is, those toward the parent root-enlarge moderately and become spherical, and thus form intercellular spaces of some size; their surfaces also become granular and their walls very thick, thus insuring the permanency of open aërial communication through the pneumathode to the tissue of the parent root, which has the most abundant system of intercellular spaces-that is, the cortical parenchyma.

Roots which have suffered metamorphosis to serve as pneumathodes have been encountered in many plants, and have been most thoroughly studied in this part of the world, ${ }^{3}$ but in all previously known cases they are formed as a response to the wetness of the environment. In many plants which grow in wet places, either frequently or invariably, pneumathodes have become normal structures; in many others, whose roots only exceptionally find themselves where the supply of air is cut off by water, pneumathode-like structures form as abnormalities. ${ }^{4}$ In plants whose habitat is such that pneumathodes have become a normal structure, the roots which serve this purpose have usually acquired a negative geotropism, adapting themselves to the direction in which the air is to be found. 'This is true of Phoenix, whose pneumathodes, as figured by Tischler, ${ }^{\mathbf{5}}$ are very similar to those of Cocos.

In distinction to all other known pneumathodes, those of Cocos are demanded by the structure of the plant without regard to what its environment may be. They form on roots in water, in firm ground, in loose sand, and in the air. In soil containing free air, where the roots normally grow and the formation of the pneumathodes is under the control of

\footnotetext{
${ }^{3}$ Karsten: Ueber die Mangrove-Vegetation im malayischen Archipel. Bibliotheca botanica (1891), Heft 22.

4 The same is true of other parts of the plant as well. See Sorauer: "Ueber Intumescenzen." Ber. bot. Gesell. (1891), 17, 456, and my note on Haberlandt's new organ on Conocephalus, Bot. Gaz. (1902), 33, 300.

5 Tischler, G.: Ueber das Vorkommen von Statolithen bei wenig oder gar nicht geotropischen Wurzeln. Flora (1905), 94, 35.
} 
natural selection, it is of course a matter of indifference whether they are above or below the parent roots; and no factor of the enviromment has the least influence in determining the place of their origin or the direction of their growth. 'They spring out at right angles, in all directions, and are straight. In water and in air they behave in exactly the same 'way. Exceptional length and negative geotropism would be appropriate reactions on the part of the pneumathodes emerging under water, but since the roots will not grow into water nor into soil without free air, their formation in this situation must be too abnormal and too rare a mischance for natural selection to have evolved any adaptation to it.

Absorption.-The same forces operate to draw water into the roots of plants which afterwards cause its movement to the leaves. There are-

(1) Suction exerted by the tissues surrounding the xylem ends in the leaves, and ultimately due to evaporation from the leaves under the influence of the sun's radiated energy.

(2) 'The osmotic activity of the cells in the roots through which the water passes. 'The former is the major factor, and its dominance is more extreme in the coconut than in most plants. This is clearly shown by two facts, the first one being that dead tips of roots for some time continue to absorb water without any measurable decrease in the rate as compared with that which was present while they were alive, and the second one is that if the tips of active, growing roots are cut off and immersed in water with not more than 5 millimeters of the cut end emerging into a saturated atmosphere, drops of water are not exuded from the cut surface; it merely remains damp. When roots are cut or broken in the ground, a gummy substance with a characteristic odor sometimes exudes, but there is neither bleeding of water nor of a dilute solution.

However, water entering the roots through the living epidermis and passing through living cells of the cortex to the stele must move under the immediate influence of the osmotic activity of these cells; a movement of the water under natural conditions is thus effected because it is constantly withdrawn from the inmost layers by suction. In this way the turgor of the roots is a factor in the acquisition of water, even in those which never bleed. The absence of bleeding only demonstrates that the living cells of the root will not pass a part of their osmotically active substance along with the water to the xylem; high turgor in the roots and abundant water in the soil will not necessarily result in root pressure.

The turgor in the pith, and in all except the fine outer cells of the cortex of the absorbing zone of the roots, equals 0.25 to 0.3 normal potassium nitrate solution. The walls are so thin that they wrinkle everywhere when plasmolysis is extreme (fig. 5). In the fine cells, which later become the hypodermis, plasmolysis is not visible in a less concentration than 0.5 normal; it is possible that the denseness of the protoplasm, together with the osmotic pressure caused by the cell sap, is responsible for this rather high figure. Plasmolysis is hard to detect in the epidermis. The turgor usually, but not always, seems to be a shade higher 
than it is in the most of the cortex-about 0.3 normal. In the roots of most plants there is a slight but not appreciably interrupted incrense in the turgor, from the epidermis inward: but this increase is no necessary condition for the ready movement of water, and in the cores we find in practien the lower turgor to be internal.

In the youngest cells of the embryonal tissue which. can be plasmolysed the limit is 0.5 normal. In the cap the turgor is for the most part 0.25 normal; and in outside cells, as long as they are alive, it is no less. All these determinations were made on roots which were apparently healthy and active.

In all my experiments on absorption by the roots homeopathic vials were used, of such a size that when filled to the proper point with water the weight was $40-45$ grams. In the cork of each was cut a hole fitting the individual root to be used. 'The latter was freed from the ground, with the least possible damage, to such an extent as to permit the necessary downward inclination of the tip. It was then washed, and all loose remains of the cap were carefully removed from the part which was to be within the bottle. 'To insure the absence of any open wounds the whole exposed part of the root, except that which was to be within the bottle, was smeared with vaseline. Water enough was used to immerse more than the absorbing region of the root, and the bottom of the bottle was kept low enough to prevent the water from touching the cork. The root, with its bottle, was laid in half of a split joint of bamboo, to which the appropriate slope was given, and the other half of the joint closed over it, thus insuring cleanliness. 'The hole in the ground was covered with abacá leaves to prevent unnatural warmth. All roots were left in this condition for one or more days before determinations of weight began.

After this time, when any initial disturbance in the rate of absorption was assumed to have passed, the hole and bamboo were opened, the bottle carefully removed, the root being touched by the bottle once to remove any free drops, and then a weighed bottle of water was substituted, the cork always remaining with the root. When all necessary care was taken to prevent wetting the cork, neither bottle needed to be open for more than five seconds, and the exposure of the root was even for a shorter time. The chief error in this method of experimentation is probably to be found in the variable amount of water adhering to the root, but experience shows that the results are reliable to a limit of 1 centigram.

The chief facts I endeavored to ascertain with regard to the absorption by the roots were the rate at which it normally takes place and the regular diurnal variation, if any, which may be found in this rate. I have also made some experiments on the absorption of solutions of potassium nitrate.

With regard to the usual rate of absorption, as has been seen to obtain for the growth, the first preliminary series of experiments demonstrated that roots which to the eye appeared to be similar behaved very differently. Nor was there correlation between vigorous growth and rapid absorption. 
I made four sets of experiments, with essentially the same results; here it will suffice to give one of these.

'I'his set was begun on January 11 and weighing commenced on January 13 ; but this beginning was made abortive by a rain which flooded the whole site. A new start was made on January $1 \%$. 'The weights given here are in centigrams, and are the average absorption for each day comprised in the interval ending at the date at the top of the column:

\begin{tabular}{|c|c|c|c|c|c|c|c|}
\hline Root No. & Jan. 20. & Jan. 23. & Jan. 27. & Jan. 31. & Feb. 3. & Feb. 10. & Mar. 1. \\
\hline I & i & ; & 6 & 7 & 7 & 3 & 7 \\
\hline II & 15 & i & 15 & 19 & 19 & 14 & 12 \\
\hline III & 19 & 8 & $a_{6}$ & 11 & 14 & ${ }^{\circ} 22$ & 28 \\
\hline IV & 8 & 8 & 2 & 3 & 9 & 8 & 7 \\
\hline V & a 16 & 18 & 28 & 35 & 43 & 43 & (3) \\
\hline VI & 7 & 11 & 10 & ${ }^{b} 3$ & 3 & 3 & .6 \\
\hline VII & a 48 & c 47 & 52 & 53 & 40 & "39 & 39 \\
\hline VIII & 6 & 7 & 8 & 9 & d 5 & 7 & 8 \\
\hline IX & 3 & 2 & 6 & 6 & $\cdot 5$ & 7 & - - - - - \\
\hline $\mathbf{X}$ & 41 & 33 & с 39 & 39 & 49 & 36 & 17 \\
\hline & $\begin{array}{l}\text { a Pneum } \\
\text { b Growtl } \\
\text { c Appare } \\
\text { a Cap slo }\end{array}$ & $\begin{array}{l}\text { thodes } \\
\text { conspic } \\
\text { tly dea }\end{array}$ & $\begin{array}{l}\text { peared. } \\
\text { ously ra } \\
\text { or dying } \\
\text { sorption }\end{array}$ & renter & $f$ & show & \\
\hline
\end{tabular}

The root $\mathrm{V}$ was injured February 10, and was then cut with a sharp knife without exposing the surface to the air, and the cut surface was then immersed just as the uninjured tip had previously been; the total subsequent absorption was only 63 centigrams. I had already satisfied myself that practically no water can be absorbed by cut leaves, and the same disadvantage from the experimenter's standpoint is presented by the roots. It is of interest to note that while an open wound is very promptly plugged, dead tips maintain their full absorbing activity for a considerable length of time.

From these results I do not believe accurate conclusions can be drawn as to the total absorption by an entire tree. The very great diversity in the rapidity of absorption by the roots is but one of the reasons for this. From a considerable number of measurements on different roots I can say that, as a general average, the end of a main root, which, on anatomical grounds, appears to be in a condition to absorb water, has about onesixth of the total surface possessed by all the root tips tributary to it. If absorption were proportional to the exposure of living epidermis, then the most rapid rate exhibited by any of these roots would indicate a total daily absorption by a large tree of only about 24 liters. But there is no such correlation between living epidermis and absorption, as is shown by the behavior of dead roots and by the two mentioned in the preceding table, the growth of which was temporarily most conspicuously rapid. The immediate result of the rapid growth was a long zone of young tissue, but in one of these cases the ensuing absorption was remarkably slow. 
The tips of the fine ultimate branches do not individually absorb with sufficient rapidity to give me trustworthy differences in weight, and they are too far apart to permit the use of several at once without a disproportionate increase in the water to be weighed. In a single instance I was able to include three of them in one bottle of the usual size, and then the observed absorption per unit of area was about three times as great as I ever found it with the tips of the main roots. No far-reaching conclusions are to be based on one fortunate observation; but it does show, as we must also conclude from the experiments to be described on transpiration, that the total absorption can be much greater than measurements made even on many tips of main roots would indicate. In one experiment, the tip of a small main root 5.5 millimeters in diameter showed a maximum rate for the time covered by eight weighings of 2 centigrams per diem.

Because of the -slight difference in weight to be determined, it was useless, in undertaking experiments to show the relative absorption during different parts of the day, to work with roots which had not already shown themselves to be among the most active. In two sets of experiments I have used such roots for this purpose. The result has always been that the greatest relative absorption was observed during the afternoon, and, so far as any conclusion could be drawn in such detail, during the latter part of the afternoon. This difference, at different hours, is usually less marked than it appears to be from the following table, which shows the results for one day with the four most active roots represented in the preceding table. The roots bear the same numbers. This experiment began at 6.15 a. m. February 1 . The figures are centigrams of water absorbed during the preceding interval:

\begin{tabular}{|c|r|r|r|}
\hline & \multicolumn{2}{|c|}{ Feb. 1. } & \multicolumn{1}{|c|}{$\begin{array}{c}\text { Feb. 2, } \\
6.15\end{array}$} \\
\cline { 2 - 4 } Root No. & 12.15 p. m. & 6.15 p. m. & \begin{tabular}{c} 
Q. m. \\
\hline II
\end{tabular} \\
V & 5 & 11 & 5 \\
VII & 14 & 21 & 16 \\
X & 10 & 19 & 10 \\
\hline
\end{tabular}

From the fact that decidedly the most rapid absorption is during and closely following the hours of most rapid transpiration, it is a reasonable conclusion that the tree contains practically no store of water on which it can easily and safely draw. However, no conclusion is justified as to the total water actually contained in the path of the transpiration stream, and therefore none as to the rapidity with which the water moves. The water may rise slowly but the demand still be propagated rapidly.

My experiments on the absorption of potassium nitrate are open to the same criticism as pertains to all of my other absorption experiments, 
namely, they had to be made on the tips of the main roots, which are not the places where the process is most active. In working with these solutions, trouble with red ants, which only exceptionally interfered with experiments with pure water, became serious; as a consequence I was finally obliged to seal all the tops of the bottle deeply with vaseline, thus completely cutting off the access of air to the water in the bottle; controls with pure water showed that during the time of these experiments very little if any interference with the absorption resulted. The investigations were made with the same roots which furnished the material for the preceding tables, and they immediately followed the conclusion of the period already reported. These results were scattered through too many days to make a tabulated report feasible. In each case the absorption of the solution is compared with that of water during the preceding period, which usually was of one day.

A solution of 0.1 normal reduced the rate of absorption for root VII from 40 centigrams (for the preceding twenty days) to 35 centigrams, which is within the limits of daily fluctuation. It was likewise questionable, in the case of main roots, whether there was any reduction by a 0.2 normal solution; for instance, with root II the rate actually increased from 14 centigrams to 15 centigrams. However, in the case of the three lateral roots, the rate fell from 51 centigrams to 16 centigrams, and after two days they were evidently unsound.

The results obtained with 0.5 normal solutions were various. With root III the decrease in absorption was only from 18 centigrams to 8 centigrams; tested again with water, the rate rose to only 10 centigrams; another application of the solution reduced it to 7 centigrams; and in water it again rose to 10 centigrams. With root $\mathrm{I}$, the previous rate having been 7 centigrams, successive determinations were 1 centigram, 1 centigram, and 2 centigrams; in water the rate returned to 8 centigrams. With other roots the half-normal solution was found to be sufficient to reverse the movement. Thus root VI, which had been very regularly absorbing about 1 centigram, lost 2 centigrams, 3 centigrams, and 2 centigrams. Root VII lost 1 centigram at one time and the three fine roots lost at the same rate.

Immediately after losing at the rate of 1 centigram for four days, root VII was put into a normal solution, and it then gained 8 centigrams in one day. This result, which at first sight was surprising, is easily explained. Water moves through the root in the direction in which it is driven by the greatest pressure. Under ordinary circumstances this direction is inward because of the influence of the atmospheric pressure, the pressure within being.less than that without. This may be expressed by stating that there is a "suction" from the inside. In using the more dilute solutions other agents must have acted together with the atmospheric pressure-agents which perhaps were put in operation by the solutions themselves; in this way the fact that the solution is absorbed will account for the result. With the half-normal solution the osmotic pressure was superior to the sum of the forces tending to make the water enter; as a result, it moved outward. Other roots may have absorbed this 0.5 normal 
solution more readily, and so have been able to keep up a slow, inward flow, for in proportion as it is absorbed it exerts no pressure. But the normal solution was sufficiently concentrated to plasmolyse all the living cells, after which it was possible for the solution to travel from outside of the root into the xylem without being compelled to pass through any of these. When this condition results there is no semipermeable membranc in its way, and, concentrated as it is, it can exert no osmotic pressure. If the half-normal solution were to cause general plasmolysis then it also would enter freely and for the same reason.

The turgor of root VI was tested. This root had lost water to the half-normal solution. A few cells in its cortical parenchyma were found to plasmolyse in this solution, but the turgor of most of them was decidedly higher-about 0.7 normal. Some cells which did not plasmolyse even in such a solution did so in a normal one. There was no active epidermis, for the hypodermis had developed so as to be only 1.4 millimeters behind the growing point, well within the adherent part of the cap. The turgor of the cap was rather below 0.5 normal. In the meristem the limit was slightly higher, but the regulation had not kept pace with that in the cortex; and in the latter it was not what might have been expected from the observations of Stange ${ }^{b}$ on the roots of various European plants.

My experiments on the absorption of potassium nitrate conspicuously show that the absorbing activity of the coconut roots is little interfered with by a moderate concentration of the surrounding solution (up to at least 0.2 normal). This obviously fits it for life in its typical habitat; for, while the water in the soil near the sea, and even in the beach itself, is not usually saline, because its mass movement is seaward, yet strand plants are subject to inundation during storms, which sometimes bring an amount of sea water about their roots which would be fatal if they were more sensitive.

\section{THE COCONUT LEAF.}

Gross morphology and growth.-Aside from the cotyledon, which is a very short sheath at one end with an enormous absorbing structure at the other, the first leaves of the coconut are mere sheaths, resembling the bases of later leaves, but entirely destitute of any lamina. These sheaths are usually 4 to 6 in number, each being longer and less scale like than its predecessor. In vigorous seedlings they sometimes appear at intervals of less than one week, but as a rule the succession is slower. Their most rapid measurable growth is immediately after they emerge from the nut. The transition from sheaths to leaves may be abrupt; or there may be one or two, the upper part of which, after splitting, bends outward, like the rachis of a leaf, but develops no blade.

The succeeding leaves, 2 to 6 in number, do not become pinnate, but develop a lamina, which splits down the median line, sometimes merely forming a notch, but usually extending more than half of the length of the

' Stange, B.: Beziehungen zwischen Substrat-concentration, Turgor, und Wachsthum bei einigen phanerogamen Pflanzen. Bot. Zeit. (1892), 50, 253, etc. 
blade. The most rapid growth of the later of these leaves occurs at a period which is a week or more after their emergence. They are plicate in vernation, but the folds are shallow and are almost or entirely smoothed out when the leaf is fully expanded. The result of this is an increase in the area of the leaf, without a corresponding growth of its margin; and this, in turn, causes it to become convex on the upper surface and to curve outward, whereby its exposure to light is materially increased, and the stomata-bearing nether surface is protected against wetting by rain. The first ones of these split leaves are apparently sessile, with blades about 20 centimeters long; the later ones are short stalked, and the length of the blades may exceed 70 centimeters.

The transition from split to pinnate leaves is a gradual one. At first only a few of the lowest folds separate, the appearance of the greater part of the lamina remaining like that of one of the merely split leaves immediately below it; in succeeding ones the pinnate lower part increases at the expense of the compact upper part until the latter ultimately disappears. The number of leaves sharing in this transition varies considerably, 6 being a common one. In length they may be from less than 1 meter to a size considerably larger. The earlier leaves are all short lived, and, as each succeeding one is larger than the preceding, their dimensions on a young tree are constantly increasing. In cultivation the nuts are germinated collectively and the seedlings set out in their permanent places during the split-leaf stage. The increase in diameter of the mass of the bases of the petioles is constant, and as the leaves have sheathing bases, as the tree grows, the latter rise into the air as a false stem, resembling that of the banana or abacá; this false stem reaches a height of about 150 centimeters before the real stem or trunk is visible. For several years after the appearance of the trunk, the leaves continue slowly to increase in number and in length. When the first nuts appear, at an age of from five to nine years, the tree is bearing at least twenty leaves. Even after this time there is usually some increase in their size; in vigorous old trees the number is 25 to 30 or even 35 ; each of these leaves is from 5 to 8 meters in length, with about 80 pairs of pinnæ, large and small.

The following table shows the rate of growth of the scales and split leaves of a number of seedlings. The measurements are from a mark on the lowest visible sheath, the husk not being dissected away; therefore there may have been some of the oldest sheaths invisible and not represented; and the growth being basal, the mark on the lowest visible sheath can record no growth. The entire elongating region is always within the protecting sheaths of the lower leaves, so that zones marked on any visible part of the leaf retain their exact intervals. Leaf No. I is the one which was marked, the others being successively younger. The numbers in parentheses represent total length; the others, the growth during the time 
clapsing since the preceding measurement. Blanks indicate no growth. All measurements are in millimeters. " $G$ " is the girth :

\begin{tabular}{|c|c|c|c|c|c|c|c|c|}
\hline Leaf No. & & Dec. 15. & Dec. 22 . & Dec. 29 . & Jan. 5. & Jan. 12. & Jan. 19. & Jan. 26. \\
\hline & 1 & $(9.5)$ & & & & & & \\
\hline \multirow{3}{*}{ I } & 2 & & (11) & 10 & 5 & 3 & & \\
\hline & 3 & 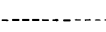 & --.---.. & (22) & 20 & 17 & 10 & -- \\
\hline & 4 & -..... & - & $\ldots$ & - & (65) & 28 & $\ldots$ \\
\hline & 1 & (11) & --.- & $\cdots$ & $\cdots$ & $\cdots$ & $\cdots$ & \\
\hline \multirow{4}{*}{11} & 2 & & $-\cdots$ & (7) & 2.5 & 1 & 1 & $\cdots$ \\
\hline & 3 & 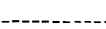 & (12) & 14 & 11 & 11 & 9 & 8 \\
\hline & 4 & 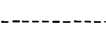 & $-\cdots$ & --------- & --- & --- & (18) & 8 \\
\hline & 1 & (11) & - & -..- & ...... & - & ....... & $\cdots$ \\
\hline \multirow{4}{*}{ III } & 2 & (37) & 10 & 4 & 2 & 2 & 2 & 1 \\
\hline & 3 & -_-_-_. & (66) & 23 & 14 & 11 & 7 & 4 \\
\hline & 4 & - - & (67) & 48 & 44 & 46 & 46 & 51 \\
\hline & 5 & & -- & --- & $\cdots$ & & $\cdots$ & (180) \\
\hline \multirow{4}{*}{ IV } & 1 & (20) & - & .... & -- & & $\cdots$ & $-\cdots$ \\
\hline & 2 & (40) & 3 & 2 & 1 & & $\cdots$ & 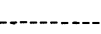 \\
\hline & 1 & (29) & --- & - & $\ldots$ &.-- & $\ldots$ & $\ldots$ \\
\hline & 2 & $(56)$ & 7 & 2 & 2 & $\ldots$ & 1 & 2 \\
\hline \multirow{7}{*}{ V } & 3 & (103) & 38 & 23 & 20 & 13 & 8 & 3 \\
\hline & 4 & $(67)$ & 39 & 36 & 41 & 53 & 38 & 29 \\
\hline & 5 & --.- & $\cdots$ & --- & (89) & 52 & 43 & 54 \\
\hline & G & (28) & 3 & 2 & 4 & 1 & 3 & 4 \\
\hline & 1 & (16) & --. & $\cdots$ & & & & \\
\hline & 2 & (46) & --- & $\cdots$ & -..- & & $\ldots$ & $\cdots$ \\
\hline & 3 & $(80)$ & $\cdots$ & $\cdots$ & ---- & -- & $\ldots$ & 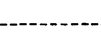 \\
\hline \multirow[t]{8}{*}{ VI } & 4 & (109) & 16 & 18 & 14 & 5 & 2 & 2 \\
\hline & $\tilde{5}$ & (120) & 51 & 53 & 53 & 48 & 51 & 33 \\
\hline & 6 & 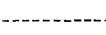 & --.- & $\cdots$ & (142) & 49 & 56 & 65 \\
\hline & $G$ & (28) & 6 & 1 & 5 & 2.5 & 4.5 & 3 \\
\hline & 1 & (5) & & & & & & \\
\hline & 2 & $(46)$ & & & -1 & & & \\
\hline & 3 & $(103.5)$ & &.- & --- & ---- & --- & \\
\hline & 4 & (204) & $\ldots$ & - &.- & & & \\
\hline \multirow{6}{*}{ VII } & 5 & (469) & 7 & 1 & 1 & 3 & 3 & 1 \\
\hline & 6 & (586) & 109 & 28 & 33 & 4 & 1 & 8 \\
\hline & 7 & (399) & 71 & 81 & 73 & 85 & 91 & 91 \\
\hline & 8 & 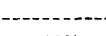 & $\cdots$ & - & & & $(352)$ & 92 \\
\hline & G & (60) & 5 & 2 & 1 & 2 & 5 & 4 \\
\hline & 1 & (38) & & & & & & \\
\hline • & 2 & $(126)$ & $\cdots$ & 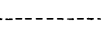 & & & & \\
\hline \multirow{4}{*}{ VIII } & 3 & (390) & - & $\cdots$ & --- & $\cdots$ & -..- & \\
\hline & 4 & $(633)$ & 25 & 6 & 11 & 3 & ---- & --- \\
\hline & 5 & $(342)^{\circ}$ & 69 & 64 & 21 & 18 & 46 & 36 \\
\hline & G & $(57.5)$ & 2.5 & 3 & & & 1 & \\
\hline
\end{tabular}

Under fair conditions each leaf of a young tree grows decidedly more rapidly than the next older one, and in seedlings which are of the size of the ones mentioned above, several leaves grow rapidly at the same time. While the plants represented in this table were under observation the growth of their roots was prevented by frequent moving. This injury was reflected by a slower development of the shoot before the measurements ceased. Each leaf had less than the normal advantage over its 
predecessor, and its period of rapid growth was abnormally short, so that in most cases only a single leaf was growing vigorously on each seedling.

Working as I did in the open and therefore largely depending on nature for modifications of the environment, it was very difficult to secure any reliable data on the influence of the individual factors of the environment on so slow a process as growth. Of the plants represented in the foregoing table, those with even numbers were watered twice daily during the first two weeks. $\Lambda$ s compared with the alternate ones, which were plared in an otherwise drier place and which received as much as 1 millimeter of rain but once in fourteen days, the growth of the watered plants wils much slower, but the relative rapidity of development was not affected by reversing the positions during the succeeding fortnight; from which it appears that the difference was inherent in the individuality of the plants, and that it is a matter of practical indifference to seedlings of the ages of the ones which I was observing whether they be given much water or very little. Observation of a seed bed where more than 5,000 nuts were placed to germinate justifies this conclusion. Differences in the exposure of different parts of this bed were not reflected in the growth of the seedlings. Until the area of the leaves permits an appreciable transpiration, the nut must contain all the water the seedling normally demands for its growth. If the husk is entirely dry the roots do not emerge from it, but this may as well be due to the extreme toughness of the dry husk as to the abnormal loss of water from the roots and to any inability on their part to absorb water. After this time a removal of the roots or a prevention of their growth by frequent moving stunts the development of the seedlings, and no amount of water will altogether obviate this result, though, of course, the injury is fatal only when excessive dryness or some other cause prevents the development of new roots. Whether the injury to the growth of the shoot of well-watered plants is correlative ${ }^{7}$ or because enough water can not be absorbed is uncertain, but in either case the leaving of the seedlings in the germinating bed after the nut's supply of water ceases to satisfy all demands, will result in injury when they are transplanted, even under the most favorable conditions.

The available moisture determines the rate of growth of the leaves of older plants to the practical exclusion of the influence of all other factors. My work on these older plants began after the influence of the dry season was seriously felt. Drought interferes first with the growth of the youngest individuals, the larger one suffering less, in proportion to the depth and extent of their root systems. The following table shows the growth of one plant (A) the development of which had practically been arrested, and of another (C) which up to the time of observation had comparatively been but little affected. In each case a leaf tip barely protruded

${ }^{7}$ Kny: Correlation in the Growth of Roots and Shoots. Ann. of Bot. (1894), 8, 265. Townsend: The Correlation of Growth Under the Influence of Injuries, Ibid. (1897), 11, 509. 
from the mass of bases. I stout stake was driven into the ground until its top was exactly even with the tip of this leaf. All measurements given are from the top of this stake and are expressed in millimeters. Increments since the preceding measurements are indicated by boldface type. The heights of the stakes were, respectively, 500 and 1,320 millimeters. The experiments began on February 8 :

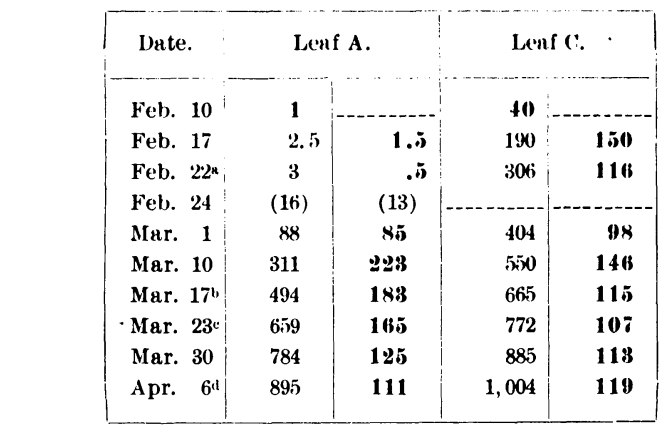

a Plunt watered. c Watered last, March 20.

b) Leaf begins to expand. d Marked part of both leaves expanded.

The following contains a more detailed tabulation of the growth of these two leaves for a portion of the time included in the preceding one and shows the relative growth by day and by night. All measurements given are the increments during the preceding periods:

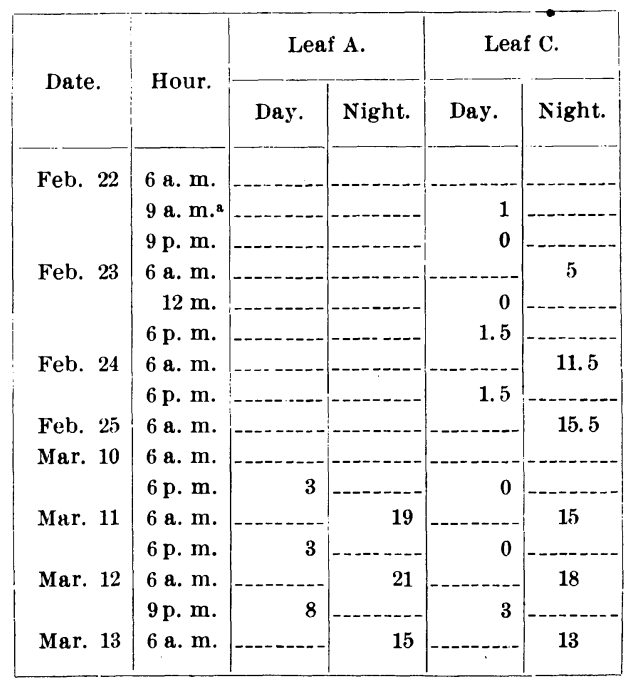

a This interval follows the first watering of leaf $\mathrm{A}$ too promptly for the growth to be at all normal.

A few measurements at other times agreed entirely with those given above in demonstrating that the measurable growth very largely took place at night, the diurnal growth of the plants which were seriously 
suffering from drought falling to nil. Indeed, a slight but unmistakable shortening occurred on certain of the days of observation. 'The reason for this strikingly unequal distribution of the growth is that the active transpiration during the day creates an internal scarcity of water and reduces the content of that liquid in the plant to such an extent that any considerable enlargement is impossible. A similar, but much less pronounced, daily periodicality of growth is reported for the bamboo, ${ }^{\mathbf{1 0}}$ correlated with the relative humidity. Every factor which contributes to the more active transpiration during the day is also in part responsible for the cessation of growth. ${ }^{10}$

It is a very common practice in Mindanao to plant coconuts and abacá together, in the expectation that the abacá will support the commercial undertaking until the coconuts mature. This may be expedient, from a business standpoint, where the cost of clearing is the chief item in the establishment of a plantation; and after the first two or three years the coconuts suffer less than the abacá in this competition; but the maturing of the former is delayed by probably two years, and the trees are never as robust as those which were better illuminated from the start. The ultimate diameter of the trunk of a palm is determined in its youth. ${ }^{\mathbf{1 2}}$

The heliotropism of the coconut is illustrated by the well-known disposition which trees along the beach have to bend toward the water (fantastically ascribed to the tree's love of the sea) and by the tendency of those around the outer edges of a grove to lean outward in every direction. This heliotropism is the more interesting because the actual growing region, where the curving takes place, is deeply seated below the visible tip and covered by the bases of many leaves.

The negative geotropism of the trunk causes a prostrate tree to turn upward with a curve the radius of which often does not exceed twice the ultimate diameter of the trunk. This abrupt curvature is rendered possible only by the harmonious reaction of many growing leaf bases, those beneath developing more and those above less rapidly than the ones in the middle. Each leaf base executes its own appropriate curve. These

${ }^{9}$ Lock: Annals Bot. Gard. Peradeniya (1904), 2, 211 . Not seen.

${ }^{10}$ Kraus (Das Längenwachsthuin der Bambusrohre, Ann. Jard. Bot. Buitenz., 1895, 12, 196), working at Buitenzorg, with almost daily rain, found the diurnal retardation of the growth of bamboo slight compared with that reported here for Cocos.

${ }^{11}$ At least the larger proportion of the experiments which are supposed to show that light exerts a direct retarding influence on the growth of stems and leaves are questionable because they do not exclude the possibility of the direct influence of the illumination on the transpiration and a consequent indirect retardation of growth. While the immediate effect of light is to retard growth, adequate illumination is of course eventually indispensable for the healty development of the plant.

${ }^{12}$ The nuts in a seed bed are usually placed horizontally because the trunks grown from such seeds are supposed to be stouter. Drude, in Natürlichen Pflanzenfamilien, 11, 3, 3, states that some palms, such as Sabal and Ceroxylon, normally develop stouter trunks if their earliest growth is horizontal. 
reacting bases are organically connected only by means of tissue which must completely have ceased to grow (it is not available for measurement), and the harmony of the entire reaction is no evidence of any 'ommunication between the units concerned in it. ${ }^{13}$

Anatomy of the leaf.-In describing the anatomy of the coconut leaf nothing need be said about the fibro-vascular tissue except that the finest longitudinal veinlets are hardly more than 0.1 millimeter apart, so that water in order to reach any cell of the parenchyma only needs to pass an exceedingly short distance by osmosis. The structure of the individual veins and veinlets offers no peculiarities.

The most striking structure in the leaf is what may be called the "hinge." Running ventrally for its entire length along each side of the midrib of the pinna is a narrow strip, sharply differentiated from any neighboring living tissue by its colorless contents. A crease along the middle of each of these strips makes the leaf thinner at this point than anywhere else, the colorless hinge tissue occupying more than half the thickness of the leaf but not entirely crowding out the green mesophyll. The epidermis of the hinge, as seen in transverse section, is remarkable for its exceedingly convex outer walls. The two accompanying figures (15 and 16) make this structure clear.

Because of the convexity of the outer walls of the individual cells, the wall of the epidermis, in this situation, as a whole is very much wrinkled; so that a bending or even a stretching can obviously be accomplished by a very slight and easy bending of walls at right angles, without giving rise to the uncompensated stretching of any one unit. Other parts of the leaf have the thick outer walls practically plane, and as any bending would involve the extension or direct compression of the whole of one of them these parts are practically rigid. Therefore, the crease mentioned above facilitates movement not only because it makes the leaf thinner at this point but also because it increases the convolution of the walls and reduces their resistance.

The active tissue concerned in the movements of the hinge is the colorless mesophyll. Its cells are large, and they have thin walls which are easily bent or even stretched. It is without intercellular space, so that the slightest alteration in the volume of the individual cells changes that of the entire tissue. The volume of the cells must obviously vary with their water content. When the leaf is well supplied with water the cells of the hinge are distended to their full capacity and it is open, thus holding the two sides of the pinna as far apart as possible. When the supply of water is insufficient the reverse takes place. By this means the exposure of the pinna to the rays from the sun or sky is lessened and a "dead air" space, though usually a very imperfect one, is formed under it. In both of these ways the further loss of water is checked.

When the pinna is losing water faster than it is being furnished from

${ }^{13}$ Cf. my paper, "The Geotropism of Split Stems," Bot. Gaz. (1900), 29, 189. 
below, the hinge responds before the cells of the green mesophyll begin to suffer. The explanation is as follows: All the cells hold their water through the osmotic activity of their contents; the turgor of the chlorophyll-bearing cells is such that plasmolysis begins in about 0.5 normal potassium nitrate, while the cells of the hinge begin to plasmolyse in less than 0.3 normal, as a consequence the latter will lose the greater amount of water in the shorter time, thus causing the hinge to close.

The actual behavior of the hinge is sufficiently illustrated by the following table, which gives the distance, in millimeters, between the edges of the two pinnæ, each measured 20 centimeters below the tip, at intervals, for two days :

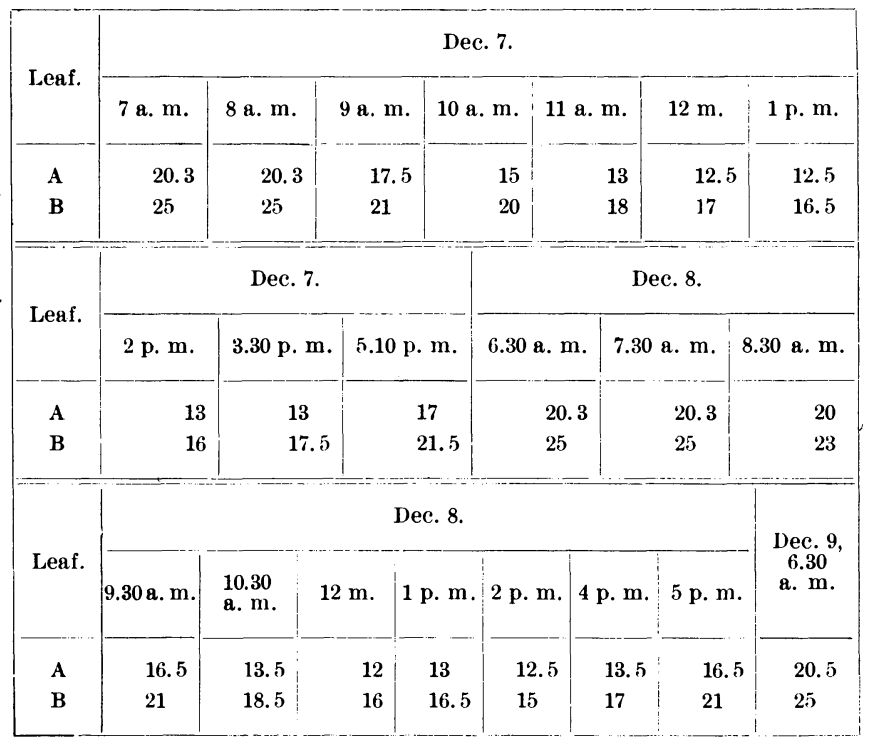

As the accompanying records show, ${ }^{14}$ the months between November and April were exceedingly dry at San Ramon. The influence of this climatic condition on the behavior of the hinge is shown by the following measurements, in millimeters, made on the same leaves at the same points one month later than the date of the preceding table:

\begin{tabular}{|c|c|c|c|c|}
\hline & \multicolumn{2}{|c|}{ Jan. 11. } & \\
Leaf. & \multicolumn{2}{|c|}{$\begin{array}{c}\text { Jan. 12, } \\
7 \text { a. m. }\end{array}$} & $\begin{array}{r}\text { Feb. 8, } \\
7 \text { a. m. }\end{array}$ \\
\cline { 2 - 5 } & 6.30 a. m. & 7.30 a. m. & & \\
\hline A & 18 & 18 & 18 & 17 \\
B & 23.5 & 23.5 & 23 & 21 \\
\hline
\end{tabular}

The surface of leaf B was moist on the morning of January 11, and this fact demonstrated that its failure to open as widely as it did a month

${ }^{14}$ See hygrometric readings appended. 
hefore had become chronic. 'This was due to the prolonged drought and not to the age of the leaf', for in seasons of fair precipitation the pinnw lose none of their original power of expansion, at least until they are much older than the ones measured in these experiments.

Aside from the hinge, the mesophyll is differentiated into the green assimilating, and what, in deference to custom, I will call a water-storing tissue. 'The latter is composed of two layers of cells, immediately beneath the upper epidermis. The upper of these layers has the cells elongated at right angles to the length of the pinnæ, as is shown by figs. 17 and 18, while those of the inner layers are considerably deeper and larger. Both are almost perfectly transparent and their contents consists almost entirely of water. They form no inconsiderable part of the volume of the leaf. 'Their walls are not sufficiently thin either to be collapsible or to stretch very easily, so that only an insignificant part of the water which they contain can ever be available to replace any loss on the part of other cells. They are primarily rather to be regarded as a screen, the function of which is to mitigate the injurious effects of too extreme insolation on the underlying green cells. The same is true of the thick-walled, socalled water-storing tissue of many other plants.

The green mesophyll is but feebly differentiated. There usually are two layers which may properly be termed palisade cells, and about four more, the cells of which are irregularly placed; but the leaf throughout is too compact for any tissue appropriately to be designated as spongy. 'The turgor of the assinilating tissue is equal to about 0.5 normal potassium nitrate.

The cells of the epidermal tissue are approximately isodiametric in surface view, their least diameter being the depth. They are devoid of chlorophyll, and hyaline. Their turgor is about that of the hinge cells, but their heavier walls prevent any considerable variation in their water content. The outer wall of the upper epidermis is 6 to $\% \mu$ thick; that of the nether, $5 \mu$. The surfaces are glabrescent.

The stomata of the coconut leaf are confined entirely to the nether surface, where they number about 145 per square millimeter. They average in size about 30 by $33 \mu$ with an area for the pores and for both guard cells of $740 \mu$ square.

As the accompanying drawing of the transverse section (fig. 19) shows, the stomatal apparatus is practically superficial, the back of the guard cell being sunk just enough to make room for a hinge in the wall outside it and to permit it to move without interference from the thick, outer wall of the epidermis. 'The mechanism is exactly that of Schwendener's type of Amaryllis. ${ }^{15} 10$ The back wall of the guard cell is so thin that it collapses and wrinkles in plasmolysis. The ventral half of each, namely, the one next to the pore, is strengthened by the powerful ridges of

\footnotetext{
${ }^{15}$ Schwendener: Ueber Bau und Mechanik der Spaltöffnungen, Monatsber. Akad. d. Wiss., Berlin, 1881, 833.

${ }^{16}$ Copeland: The Mechanism of Stomata, Ann. of Bot. (1902), 16, 339.
} 
entrance and exit and by the neighboring, comparatively heavy parts of the wall. Anticlinal walls never strike the guard cells midway. $\Lambda$ more detailed explanation of the mechanism of these stomata would be superfluous here. However, there are some interesting features about their turgor and their behavior under changes of illumination and with the gradual withdrawal of their water which render it worth while to introduce some measurements. The agent employed by me to withdraw the water was potassium nitrate. The solutions were 0.3 normal, 0.5 normal, and normal. Measurements are in microns:

\begin{tabular}{|l|r|c|c|c|}
\hline & $\begin{array}{c}\text { In pure } \\
\text { water. }\end{array}$ & $\begin{array}{c}0.3 \text { nor- } \\
\text { mal. }\end{array}$ & $\begin{array}{c}0.5 \text { nor- } \\
\text { mal. }\end{array}$ & $\begin{array}{c}1 \text { nor- } \\
\text { mal. }\end{array}$ \\
\hline Stoma: & & & & \\
Length & 34 & 35 & 35 & 34 \\
Width & 31 & 30 & 28 & 26 \\
Pore, width & 5 & 3 & 1.5 & 0 \\
Ridge of exit & 9 & 7.5 & 7 & 6.5 \\
\hline
\end{tabular}

The normal solution plasmolysed the guard cells and caused the contents of other epidermal cells to collapse until they occupied hardly half the previously visible area. Remaining ten minutes in this solution killed many of the former, and others opened the pore only after the solution was replaced by water and the slide exposed to the direct sunlight. The two stomata described below half opened in water and completely in the sunlight. Measurements are in microns:

\begin{tabular}{|c|c|c|c|c|c|c|}
\hline \multirow{2}{*}{ Leaf. } & & \multirow{2}{*}{$\begin{array}{l}\text { Direct } \\
\text { sun. }\end{array}$} & \multicolumn{2}{|c|}{ Obscure light. } & \multicolumn{2}{|c|}{ Microscope stage. } \\
\hline & & & $\begin{array}{l}15 \text { min- } \\
\text { utes. }\end{array}$ & $\begin{array}{l}75 \text { min- } \\
\text { utes. }\end{array}$ & $\begin{array}{c}10 \mathrm{~min}- \\
\text { utes. }\end{array}$ & $\begin{array}{c}15 \text { min- } \\
\text { utes. }\end{array}$ \\
\hline \multirow[t]{3}{*}{$\mathbf{A}$} & Width: & & & & & \\
\hline & Stoma & 32 & 31 & 29 & 30.5 & 31 \\
\hline & Pore & 5 & 4 & 2 & 3 & 4 \\
\hline \multirow[t]{3}{*}{ B } & Width: & & & & & \\
\hline & Stoma & 30.5 & 30 & 30 & 30.5 & 31 \\
\hline & Pore & 5 & 3 & 0.5 & 2 & 3 \\
\hline
\end{tabular}

It appears from these measurements that seventy-five minutes in quite diffuse light affects the degree of opening of the pore about as much as does immersion in 0.5 normal potassium nitrate solution. The recovery of turgescence with better illumination occurs with amazing promptness when one considers the great change in turgor which precedes and causes it. In this experiment, as is always necessary when stomata are under prolonged microscopic study undertaken with sufficient care to permit of accurate measurements, they are immersed in water. When they are in the natural condition on the living plant they respond much more quickly and thoroughly to the withdrawal of the light, as is shown by experiments to be reported below, in which the rapidity of transpiration is determined by the cobalt test. 
While all recent writers on the subject have assumed that changes in turgor are responsible for the changes in turgescence with variations in the illumination, it has never, so far as I am aware, been demonstrated that the turgor really does vary.'i I myself have tried to measure such a change with divers plants, but without success. However, with the roconut it is easy to determine that the turgor is much higher in light than in darkness, though the actual differences are rather inconstant. The turgor of a single pair of guard cells can be demonstrated to change during a prolonged experiment; but as this involves plasmolysing the pair at least twice, and, as a rule, subjecting it to several strong plasmolysing solutions, each of which must be given time to act, the cells are likely to suffer changes from this treatment. 'The evidence taken from the observation of many different stomata, in their natural condition, at different times of day, is more valuable.

'The turgor on sunny afternoons is usually about equal to normal potassium nitrate. Sometimes it exceeds even this high figure. Thus at $3.30 \mathrm{p}$. $\mathrm{m}$. November 25 these measurements, in microns, were made:

\begin{tabular}{|r|r|r|r|}
\hline & $\begin{array}{c}0.5 \text { nor- } \\
\text { mal. }\end{array}$ & $\begin{array}{c}0.7 \text { nor- } \\
\text { mal. }\end{array}$ & $\begin{array}{c}1 \text { nor- } \\
\text { mal. }\end{array}$ \\
\hline Pore, width & 2.5 & 1.5 & Closed. \\
\hline
\end{tabular}

While the particular stoma under observation was not measurably open, about one in five on the section was open to the extent of at least $1 \mu$, the plasmolysis of any guard cells being very doubtful; but when the normal potassium nitrate was replaced by glycerin, plasmolysis was evident everywhere, and all stomata were closed. If they were examined early in the morning the guard cells were usually found to have their turgor equal to somewhat less than 0.7 normal potassium nitrate but rarely below 0.6 normal. In direct sunlight the increase is an immediate one.

The action of prolonged darkness is very different from that of the mere nocturnal lack of light. A leaflet was kept in darkness, inside a wooden cylinder, for ten weeks, at the end of which time its turgor, as compared with that of a neighboring pinna under ordinary conditions, was :

\begin{tabular}{|c|r|c|}
\hline Name. & $\begin{array}{c}\text { From } \\
\text { darkness. }\end{array}$ & $\begin{array}{c}\text { From nor- } \\
\text { mal leaf- } \\
\text { let (in } \\
\text { morning) }\end{array}$ \\
\hline Epidermis & 0.5 & $0.25-0.3$ \\
Guard cells & .7 & $.6-.7$ \\
Parenchyma & $.7-.6$ \\
\hline
\end{tabular}

"The term "turgor" is used to express the osmotic pressures of the internal fluid of a cell. On the other hand, the expression "turgescence" applies to the strain resulting from the interaction of the force of the osmotic pressure (the diffusion tension of the solute) on the one hand and that of the resilience of the cellulose membrane on the other. Copeland, Ann. of Bot. (1902), 16, 330. 
In my paper, "Ueber den Einfluss von Licht und Temperatur auf den 'Turgor," 18 I showed that in leaves growing or grown in darkness the turgor is higher than in normal ones; but the pinna of the coconut which I had under observation lid not, at least in area, grow during the experiment, therefore the explanation in this case must be a different one.

'TRANSPIRA'TION.

Three general methods have been used in research on the transpiration of plants: First, measuring the water absorbed by the subject of the experiment; second, determining the loss in weight of the subject and its container; third, ascertaining the amount of transpired water after it leaves the plant. I have used all 'of these in my work on the coconut.

The first method is of the least value because it does not directly measure the transpiration, and because the amount absorbed and that given off in a given time are not necessarily equal. I employed it in some preliminary experiments only, when my equipment did not permit the use of either of the others. As the pinnæ which served as subjects always lost weight almost from the beginning of the experiments, absorption being less rapid than transpiration, the method is inapplicable when any measure of accuracy is desired. It will, of course, be understood that all ordinary means of keeping the absorption normal were employed.

In all experiments on the transpiration of this plant in which the subject is to be weighed the use of single pinnæ is practically compulsory, for even young seedlings are so heavy that the loss of water from the limited leaf area in such time intervals as one hour would escape notice. Entire leaves have the same disadvantages as do pinnæ, and besides they are most unwieldy. When it can be used at all, the determination of the loss of weight of subject and container is the most reliable method of ascertaining the transpiratory activity of any plant, and when, as in this case, the use of whole plants is impracticable, it is usually feasible, with proper care and precaution, to be sure that isolated parts of them behave, at least for some time, as they would in their natural positions. I have used this method in the larger part of my work, but, in contrast to experience with other plants, have found it quite impossible to make single, isolated pinnæ of the Cocos maintain the normal rate of transpiration for more than a very short time. Leaves cut under water, with the cut surface at all times protected from exposure to the air, approximated normal transpiration but little if at all more closely than those treated without this care. This has repeatedly been my experience. One rather extreme illustration will suffice to demonstrate it: The cut surface was never exposed to the air. The first weighing was immediately after cut-

${ }^{18}$ Dissertation, Halle, a. S., 1896. 
ting. 'The leaf was exposel to direct sunshine during the greater part of the time:

\begin{tabular}{|r|r|r|}
\hline Interval. & $\begin{array}{c}\text { Loss of } \\
\text { weight. }\end{array}$ & $\begin{array}{c}\text { Inss per } \\
\text { minute. }\end{array}$ \\
\hline Minutes. & Gram. & Gram. \\
6 & 0.15 & 0.025 \\
13 & .17 & .013 \\
50 & .26 & .005 \\
310 & .70 & .002 \\
\hline & &.
\end{tabular}

The average loss per minute during the last interval was only 8 per cent of that during the first. 'Transpiration does not usually cease so promptly, and the relative loss is less, the longer the first period is made. It is a general rule, in experiments of this kind, to permit the subject to stand for a time after cutting, and thus to become accustomed to its new conditions before observations really begin. If this is done with Cocos the rapid initial transpiration can not be observed, and thus the abnormality of the results obtained must escape suspicion. Many of my tables, which seemed satisfactory when made, are valueless on this account. When the transpiration of a leaf varies during a single half day by 92 per cent of its maximum activity, independently of any change in the environment, it is obvious that any modification of the latter must have results which are comparatively too insignificant to be studied with any confidence. Therefore, I was forced to seek a means of preventing the usual reaction to the cutting of the pinnæ.

The water in which cut pinnæ stand ceases to be clear, becoming a pale, often opalescent, brown. This is sometimes evident within half a day after cutting, but usually it is not seen until a day or more has elapsed. ${ }^{19}$

Suspecting that an exudation from the cut surface (though none was visible) might be preventing the absorption of water, I tried renewing the cut. It was doubtful if the transpiration was accelerated; certainly such acceleration was not enough to be applicable in drawing any conclusions.

It was shown by Janse ${ }^{20}$ that, while boiling a part of the path of the transpiration stream ultimately results in interference with the movement of water, this result is not immediate, and is due to changes in the part remaining alive, not in that killed. It occurred to me that boiling the bases of the pinnæ might prevent the checking of their absorption for at least a few days. As a matter of fact, the cessation was less immediate and less complete in pinnæ so treated than in others, but it was still so great that the results obtained by this method alone are far from satisfactory. They are shown in the next table.

${ }^{10}$ This is a conspicuous exception to Sachs's statement that nothing escapes from such cut surfaces into water.

${ }^{20}$ Janse, J. M.: Die Mitwirkung der Markstrahlen bei der Wasserbewegung im Holz, ,Jahrb. wiss. Bot. (1887), 8, 1 . 
The fact that neither renewing the cut nor killing the lower ends of the pinnæ prevented the practical cessation of the transpiration would suggest that this cessation is due to some reaction on the part of the stomata, but this can hardly be true, for, as alrealy noted, the cut pimma lose weight during the experiments. The only explanation I can suggest for the persistent refusal of the cut pinnæ to absorb water at least as readily as they normally secure it from the rachis of the leaf is that a pressure of less than one atmosphere within the tracheæ is a condition for the ready movement of water through them, and that offering water to the pinnæ at a higher pressure than the usual one, instead of making them absorb more, is in itself the cause of their absorbing less. I am not ready to support this suggestion here, and know that it is contrary to the generally accepted opinion that water travels through wood with equal readiness regardless of whether the motive force is applied as a pressure (above one atmosphere) or as a suction (less than one atmosphere). It seems to me that this may be true in some cases and not in others, depending, for one thing, on the amount of air in the conducting elements.

Determining the water given off by leaves by absorbing and weighing it is a method which has long been in use. A decade ago, in a paper not accessible to me at the time I carried out this work, Stahl introduced the use of anhydrous chloride of cobalt, the rapidity of transpiration being estimated by its change in color from blue to pink as the salt absorbs water, because cobalt salts are blue when anhydrous, red when hydrated. As standards I used pieces of absorbent paper saturated with cobaltchloride solution, one set not quite as blue as it would be if entirely anhydrous, the other not as red as if entirely hydrated; these sets were separately sealed in glass vials. While changing from the color of one of these to that of the other, a piece having an area of 100 square centimeters absorbed 0.46 gram of water. By the use of this cobalt paper the transpiration of pinnæ in their natural positions on the tree could be tested, the evil effects of cutting being entirely obviated. However, the method has compensating disadvantages.

The cobalt paper must be directly applied to the transpiring surface, and it must be protected against the possibility of absorbing water from the atmosphere. This is accomplished by holding it in place with glass (microscope slides serve the purpose well), the latter in turn being held by clamps. This method is likely to make the transpiration abnormal by interfering with the wind, by cutting off some of the illumination, and by placing a portion of the leaf, at least for a part of the time, in an abnormally dry atmosphere.

Transpiration is exceedingly sensitive to changes in the illumination, so much so that if a slide which is locally corroded be used over either surface, the paper under the etched spot will be noticeably slower to turn red; therefore clear and perfectly clean glass must be used. However, 
there is no avoiding interference on the part of the cobalt paper itself; but nearly all of the light to which the plant has access comes from above, and the disturbing effect of cutting off that from below is correspondingly moderate.

'The wind affects the transpiration in two ways-by constantly changing the air immediately outside the stomata and by agitating the leaves and thus causing a circulation within the intercellular spaces and an egress and ingress through the stomata. The disturbance of the first of these effects is inoperative in this case because of the drying action of the cobalt paper; and as the pinna as a whole remains fairly movable, only the small part between the slides being rigid, and the intercellular spaces are continuous, the interference with the circulation is at most but partial. The disturbance of the transpiration by cutting off the wind is therefore not a serious matter.

That the cobalt-chloride test of transpiration places the leaf in an abnormally dry atmosphere is a great and unavoidable objection. Even if the plant did not react to this condition other than as a surface of water would, namely, by more rapid evaporation, this error would be very difficult to control ; for while the blue paper must constantly surround itself with very dry air, this medium becomes damper as the paper turns red. ${ }^{21}$ In practice, the matter is far from being as simple as if we were studying evaporation from a water surface only. When the cobalt paper is applied to a surface with open stomata it suddenly makes an increased demand on the water vapor in the intercellular spaces which are in immediate contact with the open pores, and most particularly on the water in the guard cells themselves. An abnormally active escape results, this in turn causes the stomata to close, checking the loss, and this process presently brings the transpiration below the normal. Thus, the decreased illumination and abnormal dryness work together in reducing the transpiration, and their combined effect is to cause the paper to change color, at first more rapidly than the normal transpiration would make it do so, but afterwards much more slowly.

That the rapidity of reddening of the cobalt paper comes far from indicating the actual rapidity with which the plant loses water is clearly shown in the last preceding table, in which the time intervals are those required for the reddening to take place. As the first column shows, the initial reddening took place in one-fiftieth of the time consumed in the last interval. The second column shows the relation of the reddening to the actual, though abnormal, transpiration. The area of this pinna was 75.4 square centimeters. Of this, 19 square centimeters was under the slide, leaving 56.4 square centimeters free; 56.4 centimeters of cobalt paper would absorb 0.26 gram of water in changing color. Evidently

\footnotetext{
${ }^{21}$ Very soon after paper in contact with actively transpiring leaves is really red, water begins to precipitate on the glass.
} 
the cobalt paper withdrew water from the leaf during the first two periods more rapidly than it transpired from the free surface but much less rapidly during the last period. The change from an acceleration to a retardation occurred in about half an hour; it often appears more quickly. The measurement of transpiration by weighing cut pinnæ and their container was deemed unavailable when this experiment showed that the rate fell during a few hours to 8 per cent of the initial. As determined by the cobalt test, the rate fell during the same experiment to less than 2 per cent. This exhausts the really distinct methods of making continuous direct determinations of the transpiration of a single subject.

Neither of these usually reliable methods being alone available in working on the coconut, I next had recourse to combinations, attempting to check a continuous experiment with one method by applying frequent corrections obtained from observations by the other; thus at the same time having the advantage of accuracy in the weighing method, and that of working with uninjured pinnæ on the tree by the cobalt test. I first tried to reach these ends by determining at intervals of several days the loss of weight of pinnæ placed in bottles of water, and at the same time comparing the rate at which cobalt paper was turned red by these cut pinnæ with that at which it was altered when it was applied to pinnæ in situ on the tree. My most satisfactory experiments of this kind furnished material for the following table:

The pinnæ $\mathrm{A}, \mathrm{B}$, and $\mathrm{C}$ were cut on the afternoon of January 17 and the cut ends killed by insertion in nearly boiling water. The leaf $D$ was freshly cut at 2.30 p. m. January 18, and its ends not killed. All weights are in grams. The loss was determined regularly at one-hour intervals during the day. The bottles were hung in the tree, putting the pinnæ as nearly as was possible in natural conditions. The bottles themselves were shaded to prevent heating.

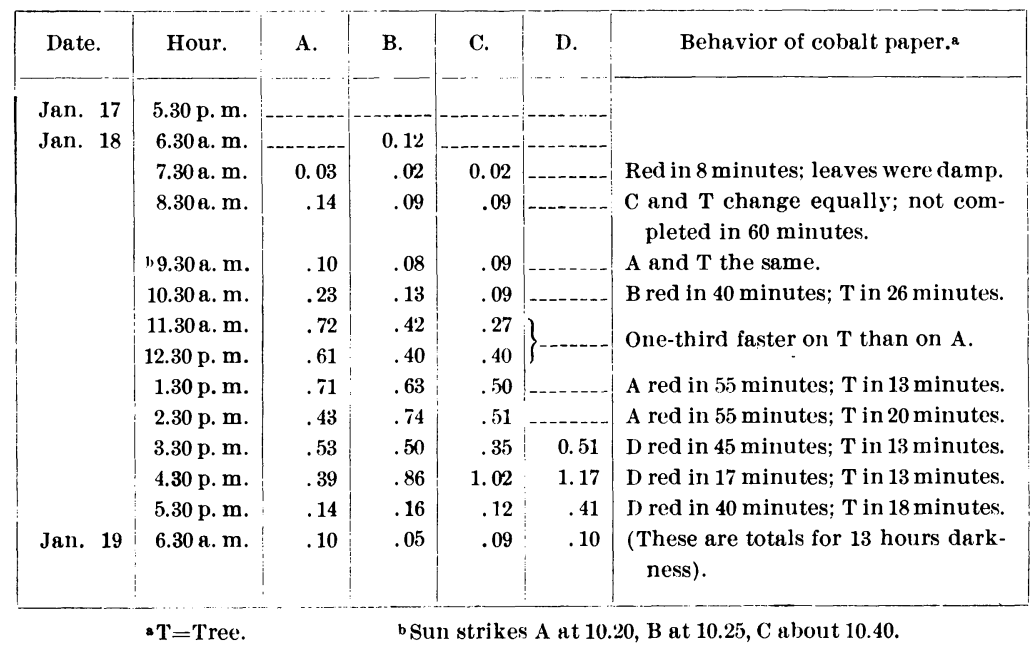


During the following day the transpiration was much slower and too alonormal to be worth reporting in detail. 'The totals for the twenty-four hours ending January 19 and January 20 were:

\begin{tabular}{|r|c|c|c|c|}
\hline Ending- & A. & B. & C. & D. \\
\hline Jan. 19 & 4.13 & 4.10 & 3.55 & \\
\hline Jan. 20 & 1.58 & 1.71 & 1.33 & 2.02 \\
\hline
\end{tabular}

If now the transpiration of a pinna in silu be computed from the loss of weight by pinne in water, and the relative rapidity with which these and the former turn cobalt paper red, the following result is obtained:

\begin{tabular}{|c|c|c|}
\hline Hour. & Grams. & Remarks. \\
\hline 7.30 a.m & 0.03 & Observed for A. \\
\hline 8.30 ค.m & .14 & Do. \\
\hline 9.30 я.m & .10 & Do. \\
\hline 10.30 ค.m & .23 & Do. \\
\hline 11.30 a.m & .96 & $4 / 3$ by 0.72 \\
\hline 12.30 p.m & .81 & $4 / 3$ by 0.61 . \\
\hline 1.30 p.m & 3.02 & 4.25 by 0.71 . \\
\hline 2.30 p.m & 1.19 & 2.75 by 0.43 . \\
\hline 3.30 p.m & 1.78 & 3.5 by 0.51 . \\
\hline $4.30 \mathrm{p} . \mathrm{m}$ & 1.53 & $17 / 13$ by 1.17 \\
\hline 5.30 p.m & .91 & $22 / 9$ by 0.41 \\
\hline Night_. & .10 & Observed for $\mathrm{A}$. \\
\hline Total & 10.30 & $\underset{\text { day. }}{\text { For one pinna and one }}$ \\
\hline
\end{tabular}

a The free area of leaves furnishing this figure averaged about 120 square centimeters, the rute therefore equaling 8.57 grams for 1 square decimeter. Haberlandt (Anatomisch-physiologische Untersuchungen iiber das tropische Laubblatt, Sitzber. Wiener Akad. (1892) I0I, 1; 804, 807) found a rate for Cocos at Buitenzorg of 0.89 gram per diem per square decimeter of surface.

Allowing 150 pinnæ to the leaf and 25 leaves to the tree, this indicates a total daily transpiration for the tree of 38,551 grams. My estimates made in this way have ranged between 28 and 45 liters. These calculations are based on determinations made on sunny days, and some of them are doubtless higher than the average transpiration of the tree for all days. On the other hand, it is to be observed that no allowance is made for the fact that not all parts of the pinnæ under experiment were free to transpire.

Another way of combining the cobalt test with the weighing method is to use fresh leaves at frequent intervals. This combination offers the advantage that the transpiration of the subjects weighed is never very much below the normal, but the disadvantage that it is difficult, with such a frequent change of subjects, to apply a control based on the continuous use of the same pinna. In practice, if the cobalt paper is always applied to a fresh part of the pinna, it will turn red once or twice after the pinna is cut, at practically the same rate as before. Under ideal conditions this method will furnish really accurate results, but the test of a method is 
its usefulness under unfavorable circumstances, and under them the lack of continuity becomes too great an objection; in addition, the observations demanded at short intervals on several pinnæ at once require a dangerous haste in manipulation. This method is serviceable where immediate results will answer, as, for example, in testing the effect of shading. Estimates of the total daily transpiration by this method, based on determinations made in the sun, run higher than those just given-sometimes as high as 75 liters per diem.

After this necessarily prolonged discussion of method, a brief consideration of the relative transpiration from the upper and nether surfaces of the leaves, the influence of their age on their transpiration, and the effect exerted from without by the illumination and the wind will be possible.

Almost the entire transpiration of the coconut is through the stomata of the nether surface of the leaf. In experimenting on the transpiration from the upper surface, and at no other time, have I found it necessary to seal the edges of the glass slides to prevent interference by the moisture of the atmosphere; of course, it was also necessary to guard against the passage of moisture from the nether surface to the upper. These precautions being taken, it requires at least six hours of continuous sunshine to enable the cobalt paper to change color. If the leaf is placed in the shade or in the dark, the hydration is somewhat slower. On January 21, a day when there were occasional clouds, the average time of reddening, when the paper was placed against the lower surface, was eleven minutes, but against the upper, seven hours; this interval, from 9 a. m. to 4 p. m., was required for the change; and paper blue at 11.25 was still of the same eolor at 5.30 , but red at 8 .

Experiments were made on the transpiration of leaves which were just full grown, those about six months older, and those a year beyond maturity. Two series of determinations were undertaken with the individuals of each age. These varied in detail, as is true with all of this work, but the relation was constant-the leaves six months beyond maturity transpired rather less than those which had just grown, while those a year old were decidedly the most active of all. For example, the total transpiration for seven hours, from 9.20 a. m. to 4.20 p. m., February 14, was-

\begin{tabular}{|c|c|}
\hline \multirow{2}{*}{ Mature leaf } & Grams. \\
\hline & \\
\hline (1) & 3.37 \\
\hline
\end{tabular}

The result for the leaf of mean age is too small; this is due to its being the first to become greatly abnormal. 'The totals for $4 \frac{1}{2}$ hours, from 1.50 to 5.20 p. m., February 15, were-

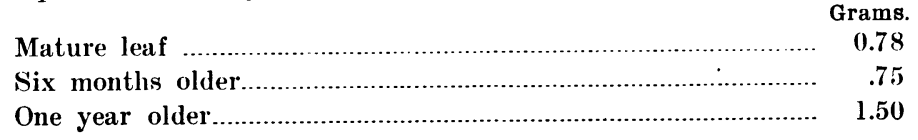


When I first observed this phenomenon I was surprised that the oldest leaves showed the most active transpiration, but a few weeks later a paper by Bergen ${ }^{22}$ was received showing that the coconut is not peculiar in this respect. The figures given above are not corrected for area; if this were done it would still further emphasize the difference, because in both cases the oldest leaves had the least exposed area. The relative activity of cut pinnæ and those in situ was at first exactly the same in all cases and so demanded no correction, but the oldest leaves were always the last to show an extreme depression. The transpiration from the upper surface was slightly more active in the latter, but it was not enough so to account for any great proportion of the extra quantity. A considerable part of the total area of the oldest leaves was occupied by small, scattered brawn spots, and the leaf was dead two months after these observations. The tree was a young one.

Thirty or more determinations of the transpiration during the night have all shown concordant results, the rate being about 1 per cent or even less of the greatest: during the day; the total transpiration for an entire night was about one-tenth of that during one hour of sunlight at midday. Three factors are responsible for this great nocturnal depression-the darkness, the lower temperature, and the higher relative humidity. The complete experimental analysis of these three factors was practically impossible, but the cobalt test, being independent of the moisture of the environment, is capable of showing the inflence of the illumination independently of the relative humidity.

By this test it has repeatedly been proven that a very slight shade will to a certain extent almost immediately depress the transpiration. Of course, actual darkness has a very much greater effect. In using the cobalt test I held the glass slides to the leaf with cork clamps, and therefore the spot immediately between these was in approximate darkness. When, in the first test, the paper reddened in about four minutes, the change was not appreciably hindered by the cork; but if this first test required more time, and always during subsequent tests, the darkened part of the paper was very evidently slower in turning. Beginning at 9.18 a. m. January 21 the intervals required for reddening were ten minutes and nine minutes; then, with a light haze over the sun, fifteen minutes; all these for the illuminated part of the paper. After the last determination, the paper was left until the part under the cork was reddened; in about forty minutes water was precipitating on the glass over the lighted leaf, but the darkened paper was still bluish, only becoming as red as the standard after ninety-five minutes - that is, it took more than six times as long to change as it did when a mere haze weakened the light.

${ }^{22}$ Bergen, J. Y.: Relative Transpiration of Old and New Leaves of the Myrtus Type, Bot. Gaz. (1904), 38, 446. "The leaves of six out of the eight species studied transpire more for equal areas when fifteen to eighteen months old than they do when they have just reached their maximum area (i. e., at three or four months)."

$36540-3$ 
In observing another leaf, the intervals at the same time were respectively fourteen and eighty-two minutes, the ratio being practically the same. The effect of the passing of a cloud before the sun was observed very many times; it naturally varied with the depth of the shading. In similar cases the test by weighing shows a depression in transpiration, but I could detect no additional one to be ascribed to the higher humidity.

It is clearly in large part because the direct sunshine heats the leaf above the temperature of the surrounding air that the transpiration is so much more rapid in it than in the brightest diffuse light. The following table shows the extent of this overheating. The temperature was determined by tying a leaf backward around the bulb of a thermometer:

\begin{tabular}{|c|c|c|c|}
\hline \multirow{2}{*}{ Hour. } & \multicolumn{3}{|c|}{ Temperature. } \\
\hline & In shade. & In sun. & In leaf. \\
\hline & $\circ$ & $\circ$ & $\circ$ \\
\hline 7 a. m. & 20.3 & 21.8 & 21.9 \\
\hline 8 a. m. & 24.3 & 25.2 & 27.4 \\
\hline 9 a. m. & 26 & 30.7 & 33.1 \\
\hline 10 н. $\mathrm{m}$. & 26.9 & 32 & 35.4 \\
\hline 11 a. $\mathrm{m}$. & 27.8 & 31.5 & a 34.7 \\
\hline $12 \mathrm{~m}$ & 28.3 & 34.7 & 37.7 \\
\hline 1 p. $\mathrm{m}$. & 28 & 30 & ${ }^{\mathrm{b}} 31.5$ \\
\hline 2 p. m. & 28.5 & 31.5 & 38 \\
\hline 3 p. m. & 28.8 & 31 & 36.7 \\
\hline 4 p. m. & 28.6 & 30.6 & 36.4 \\
\hline 5 p. m. & 27.7 & 30 & 34 \\
\hline 6 p. m. & 26.6 & 27.6 & 28.5 \\
\hline
\end{tabular}

How great a difference in evaporation, as a merely physical process, these differences in temperature will exert is shown by a consideration of the variation in the tension ${ }^{23}$ of water vapor with changes of temperature. Thus, at noon the temperature in the shade was $28 .^{\circ} 3$; at this point the tension of water vapor is 28.560 millimeters; at the temperature of the exposed leaf, $37 .^{\circ} 7$, the vapor tension is 48.463 millimeters; at 11.30 a. m., with a temperature $28 .^{\circ} 4$, the relative humidity was 66 . The tension of vapor in the air at that time was 18.89 millimeters, making a relative humidity for the temperature of the leaf of only 39 ; the unsatisfied possible tension of vapor in the air was 9.69 millimeters in the shade, while it was 29.583 millimeters for the leaf.

The actually observed excess of transpiration in strong, direct light over that in the shade was greater, as a rule, than that of evaporation from a water surface under the same temperature conditions; the change from a light haze, under which the leaf is already somewhat overheated, to full illumination, frequently multiplying the rate of transpiration by four. This extra effect may in part be due to the action of the stomata, and must in part be ascribed to the expansion of the gas in the intercellular spaces, with the consequent ejection, as the leaf is warmed, of a portion

${ }^{23}$ Tables of Landolt and Bernthsen. 
of this gas loaded with moisture. Of course, the opposite change in the volume of this included air would take place as the leaf cools.

It was impossible for me to make observations of any great value on the influence of the wind, because I could not regulate or measure its velocity. With a good subject, the concomitant use of the cobalt test and the weighing method should make it possible accurately to analyse the wind's influence, showing how much is due to mechanical agitation and how much to the constant change of the air outside. But no work on the coconut is sufficiently accurate and reliable for such an analysis. As was to be expected, the wind made a much greater difference in the transpiration of the leaves which were exposed to the greatest illumination than it did in that of the shaded ones. Thus, in one instance, the transpiration in direct sunshine was four times as great in a wind I estimated to be at 5 miles an hour as it was in a calm; but the increase was usually not more than 100 per cent. In the shade, a wind of this velocity added less than 50 per cent to the transpiration. . I was unable to cut off the wind from a shaded plant. without further interference with its illumination.

Any estimate of the total water transpired by entire trees can not be more than a rough approximation, because, aside from all possible inaccuracies in the observations on individual pinnæ, different days and seasons are unlike; and different neighboring trees, as well as different parts of the same individual, interfere with each other's transpiration. For these reasons any estimate based on observations made entirely in direct light must be too high. As already stated, some calculations obtained in this way are as high as 75 liters per diem. In the experiment from which the estimate of 28 liters was obtained the pinnæ were under as normal conditions as possible, taking their share of shading with the other pinnæ of the tree and being under check by observations on pinnæ in the natural position. The day was bright, but was not quite cloudless, and not especially warm.

At the rate of 28 liters per diem the annual transpiration is 10,220 liters. In this volume of water the plant takes up the mineral food to be used in its permanent growth and enough more to cover the annual loss in the nuts and cast leaves. The amount of mineral food permanently bound up in the growth of the stem and roots can not be very considerable, and that in the roots which die is already in a place to be absorbed again. The average dry weight of a fallen leaf may roughly be put at 3 kilograms, of which 8.5 per cent is ash and nitrogen. Allowing a fall of 16 leaves per annum, the loss of matter taken up in solution by the roots is 4,080 grams. In each nut the tree loses ash as follows:

In the husk.

In the shell..

In the copra

In the milk 
If the tree produces but 20 nuts per annum, which is more than the recent average at San Ramon, the loss of mineral matter in these is 1,140 grams and the total loss in leaves and nuts 5,220 grams. If this were absorbed in 10,220 liters of water the concentration would be 0.051 per cent. This is considerably above the average concentration to be found in ground water, as determined by analyses from water in wells and springs, but as a general proposition the water in intimate contact with the ground particles, and, when there is but little water in the soil, all of it, will be more concentrated than that which will run freely from wetter ground; and the valuable mineral food of plants is absorbed from such dilute solutions in greater proportion than is the water in which it is dissolved.

Effect of drought.-The season during which I carried on my work at San Ramon was characterized by extreme dryness, and this condition has interfered with my study of the plant's normal physiology, but at the same time it has given me an opportunity to observe the injury done by the abnormal conditions. The following table contains my measurements of the rainfall for this year and Mr. Havice's for the corresponding months a year ago:

\begin{tabular}{|c|r|r|r|}
\hline Month. & $1904-5$. & $1903-4$. & $\begin{array}{c}\text { Relative } \\
\text { humidity, } \\
1904-5 .\end{array}$ \\
\hline November & mm. & mm. & Per cent. \\
December - & $(15-30) 91.5$ & $(1-30) 208$ & 79.9 \\
January - & 2.5 & 260 & 79.2 \\
February - & 30 & 32 & 75.65 \\
March & 1 & 431 & 72.35 \\
April & 0 & 232 & 75.43 \\
\hline
\end{tabular}

The third column gives the average relative humidity at or near the beach at $11.30 \mathrm{a} . \mathrm{m}$. for November and at noon for the remaining months. Details as to the rainfall and humidity during my work are presented in the appendix to this paper. While the dryness of the air certainly has some direct effect on the coconut trees-for example, in influencing the movement of the hinge, without regard to how well the roots may have been supplied with water-I do not believe that serious damage is ever done to the tree except by the dryness of the ground. In other words, trees judiciously irrigated have nothing to fear from a drought, however severe.

The cultivated part of the San Ramon farm is well supplied with ground water, which, as a rule, finds the surface through a number of large springs. Two months after the drought began, some well-cultivated spots were still wet from below every morning. During November, December, and January, I frequently examined the young tips of roots, and through these months there was no important change in the condition of the ground and accordingly none in the roots. After the latter 
part of December there was a rapid drying lack of the streams running to the farm from the mountains, and the desiccation of the ground became rather abruptly evident a month later. 'Through January, surface cultivation kept all but the most porous ground in good condition, but after this time it was practically useless so far as the soil was concerned. By the middle of March the soil, where it was not sandy and ready to crumble, was as hard as if baked, and under the thoroughly cultivated surface it was full of fissures as much as a centimeter in breadth. 'The hardness was shown by the behavior of main roots nearly 1 centimeter in diameter, to whose disposition to grow in a straight line the tree owes its firmness. These, upon entering the cracks, turned almost at right angles and started to follow them.

In such a soil it is obvious that, in a short time, growth will be suspended. On March 21, I was unable to find any roots apparently normally active. The cessation of growth had been accomplished, or initiated, by the shortening of the growing region until the hardened hypodermis had advanced to within the root cap, obliterating the white absorbing surface. The disappearance of the absorbing region in the small branch roots, with short caps, was at first less complete, but by April 11 that portion remaining unlignified at the tip even of these was more or less flaccid, even in the early morning.

The turgor in the cortex of these roots equals nearly 0.4 normal potassium nitrate. Approaching the meristem it is higher, probably 0.5 normal. In the cap and epidermis I was unable to determine it. It will be noticed that the increase in turgor caused by desiccation and cessation of growth is more than half what it is when the cessation of growth and immersion in 0.5 normal potassium nitrate act together. This shows what proportion of the increase in the latter is directly due to the absorption of the salt. ${ }^{24}$

Of course, the roots of a tree do not all suffer alike, because different strata of the soil do not become equally dry. I tested the amount of moisture in the soil on April 11, at depths of 20,60, and 100 centimeters, determining the weight lost by drying at a temperature of $40.3^{\circ} \mathrm{C}$., the dew point being $25.5^{\circ} \mathrm{C}$. The loss was-

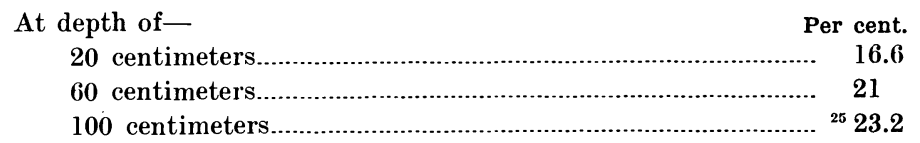

${ }^{24}$ For the influence of rather concentrated solutions on the turgor of immersed roots, see Stange, in Bot. Zeit, 1892. For the influence which the mechanical prevention of growth exerts upon the turgor, see especially Pfeffer, Ueber Druckund Arbeitsleistung, $\cdot 1893$. For a general treatment of the dependence of the turgor upon the rate of growth, see my paper, Ueber den Einfluss von Licht und Temperatur auf den Turgor, loc. cit. 1896.

${ }^{25}$ The difference in available water is much greater than these figures would indicate, for at 20 centimeters in depth the soil is the hard clay already mentioned, while at 100 centimeters it is a sandy loam, crumbling readily; at 60 centimeters it is intermediate in character. 
A very large part of the roots of the coconut grow in the stratum between 20 and 50 centimeters, and a tree to which water is available only at a greater depth must suffer. If conditions exist which permit roots to grow only at a greater depth, the obvious result will be a larger proportion in this deeper situation; in this way trees growing in dry places adapt themselves to their environment. However, trees which are compelled to adapt themselves to unfavorable conditions of any description can not be expected to be prolific. This is well illustrated by the dearth of nuts on the Cocos-clad hills about Romblon and Masbate. The water which can be drawn from a dry soil contains a greater proportion of mineral substances dissolved in it than that which is available when the soil is wet, so that the proportion between the quantity of available mineral food and the amount of water absorbed is not constant.

The shoot suffers from the inactivity of the roots. The influence of the drought on the growth of the leaves, and on the action of the hinge, has already been shown. The leaflets, which under these conditions are more folded, absorb less light, so that the leaf area which the tree has at its disposition is less efficient in photosynthesis. A normally active tree produces from twelve to twenty-four or more. leaves a year. After December, during this drought, no new leaves appeared on trees which were less than 2 years old, and not more than one on any tree less than 5 years old. As a general rule, the older the tree the later it begins severely to suffer, the probable cause being that its roots run deeper than do those of the younger ones; but the growth of the leaves of individuals of all ages was very evidently retarded during February. This, in itself, would result in a decrease in the number of leaves borne at one time; but another factor is at least equally efficient in bringing about this result. The old leaves of the coconut are cast in a succession which, in adult trees, normally keeps pace with the appearance of the young ones, so that the number present at any one time does not materially vary. The internal factors causing the fall of the leaves have never been investigated, but there is no doubt that dryness is one of them. The "physiological dryness" caused by the outside drought naturally finds expression in a more rapid aging and falling of the leaves. In fact, the first, and for a long time the only, noticeable symptom of dryness is the number of leaves pendant or falling. It has already been noted that trees without a rather indefinite minimum number (say, twenty) of leaves, have not the vitality which is necessary to ripen nuts. An individual with only approximately this number will naturally not bear many. A retardation in the production of leaves and an acceleration in their loss, when acting together, will rapidly bring even the strongest trees toward this limit.

The flowering branches are formed in the axils of the leaves, and the formation of fewer of the latter must in itself ultimately result in the growth of fewer of the former. However, in practice, the development of these branches themselves is dependent, like any other growth, on a 
sufficiency of water; it is arrested at the same time as is that of the younger leaves, long before the ones formed during the drought would bear flowers on their axillary branches, even in the most favorable weather. 'Thus the number of branches whose pistillate flowers were "opened" on a certain tree during the six months ending with April, 1905, was six, whereas during the preceding six months it had been nine. The flowers do not open until more than six months after the first appearance of the subtending leaf.

'The number of nuts which can be borne depends upon the number of fruiting branches, and on every branch there are more pistillate flowers than can possibly give rise to mature nuts. The number which develop is a matter of individual difference between the trees; some regularly bear as many as ten, others never more than three. My observation of mature trees has not shown that the drought exercises any influence on the number which blast. It has seemed to me that in a grove in which the trees are in the first year or two of bearing a somewhat larger proportion than usual was blasting during the drought, but then it is also true that a very large percentage always do blast on such trees (on the first branches no nuts mature), so that this effect is uncertain. Neither have I been able positively to determine that the drought exerted any influence on the rapidity of the ripening of the nuts. If there is such an influence it will be toward a more rapid ripening, the tree thus producing smaller nuts, with less store of food. 'The records of the San Ramon farm show the number of nuts cut and the number of nuts and amount of copra sold, but they do not show how many nuts have been picked up from the ground nor at what times nuts have been used for seed; and these items are so considerable that I can draw no sufficiently accurate conclusions as to the yield of copra per nut. ${ }^{26}$

The direct result of the checking of the growth of the young leaves and flowering branches will be a deficiency in the yield of nuts, beginning not less than nine months after the drought first makes itself felt (nine months being about the minimum time between pollination and maturity) and ending at least eighteen months after the drought is broken (that being the usual time elapsing between the appearance of a leaf and the maturing of the subtended nuts).

There are other considerations which make it necessary to extend this period of depression in both directions. When more than a minimum number of nuts are borne on a branch the latter itself is unable to sustain the weight, so that the additional support must be furnished by the petioles of the lower leaves. The untimely casting or depression of these leaves withdraws this support and leaves the branches carrying the greatest load in a condition in which breakage is likely to occur. The nuts

\footnotetext{
${ }^{28}$ Judging by the eye alone, I can say positively that the nuts cut during April, 1905 , averaged distinctly smaller than usual.
} 
are heaviest about three months before maturity, and the loss by premature falling becomes considerable within five months after a drought first becomes serious. At about the same time the drought makes itself felt in injury to the crop through another channel. At all times some nuts are destroyed by crows, but the loss is usually inconsiderable. However, in a period of drought, when other food is scarce and the water courses are dry, they concentrate their attention on the young coconuts and accomplish no little destruction.

The injury to the tree's vitality during a prolonged drought is so severe that the return of favorable weather conditions is but slowly followed by the resumption of the normal activity. When rains come, the roots must awaken from a state of defensive rest in which a prompt response can not be expected. The partly folded condition of the pinnæ, induced by the dryness, seems permanently to remain; at any rate, recovery from it is very slow. A tree which through unfavorable conditions has only twentyfive leaves remaining has not the strength, even under the best conditions, at once to return to the formation of new leaves at the rate which is necessary for the maintenance of a head of thirty. Recovery after a drought is a building-up process, and it must be a slow one. It can hardly be complete in two years, and the return to the normal crop of ripe nuts which can be produced during uninterrupted good seasons can only be well under way in this time.

There is no record of the rainfall at San Ramon prior to September, 1903. The beginning of that year was a period of drought, like the one which has characterized the early months of 1905, but the former can not have been as intense as the latter, for the springs did not so completely disappear. The following record of the number of nuts cut shows how gradually this drought made itself felt and how prolonged its effects have been. ${ }^{27}$

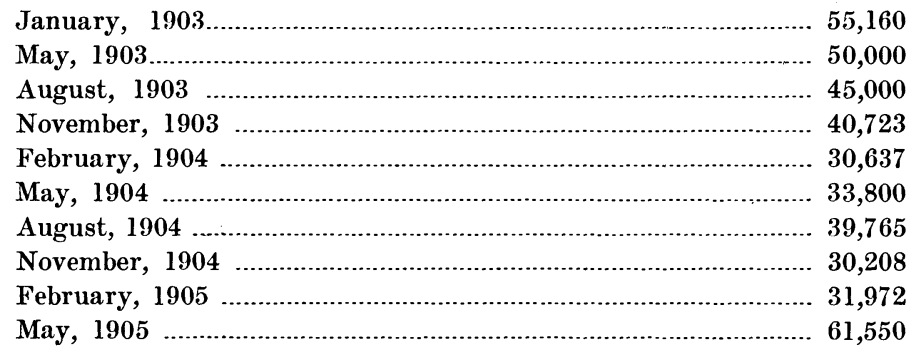

The report of the superintendent of the farm for June, 1902, states that there were at that time 5,722 coconut trees in bearing on the farm and that 1,809 trees should begin to bear within two years from that date.

\footnotetext{
${ }^{27}$ The nuts are cut every three months. The work is done by contract, at the rate of 2 pesos per 1,000. This record is made from the "general-expense vouchers" for the expense of cutting.
} 
With this increase in the number of trees, without any improvement in their yields, if there had been no drought, the total cut of nuts for 1904 should have been over 300,000 ; the actual number was 134,410 . 'The record just given shows that the period of depression which followed the former drought was identical in character with the one to be anticipated from the present condition of the trees. 'The first real step in return to a fit yield was the cutting of May, 1905, about two years after the former drought ended; the return can not go much farther before the effects of this drought will head it off.

It is then the experience at this farm that a "dry season" occurring only every other year will constantly keep the yield of nuts at considerably less than half of what it would be if the supply of water were always sufficient for the tree's needs. It is obvious that a coconut plantation will be a probable source of continual profit only in localities where dry seasons may never be expected or where it is feasible by irrigation always to keep the ground sufficiently moist to enable the roots to preserve their full, normal activity.

\section{CONCLUSION.}

We have just seen that a considerable supply of water must constantly be at the disposal of the coconut, or it will protect itself against injurious desiccation by a partial suspense of its vitality. The necessity of this water as the carrier, in solution, of the plant's mineral and nitrogenous law food has previously been touched upon. I made no direct experiments in the fertilization of the coconut, but it is the unanimous experience of those who are acquainted with the subject that an increase in some of the constituents of its mineral food has a very marked favorable effect on the production of the fruit. ${ }^{28}$ At San Ramon certain trees are pointed out as particularly productive because they have long received the waste from the kitchen. The quantity of mineral food which the tree takes is roughly proportional to the amount of water which it absorbs. ${ }^{29}$ Increasing the plant's transpiration has, then, the same effect

${ }^{28}$ Experiments with the object of determining whether the soil surrounding the coconut roots contains nitrifying organisms were undertaken by Dr. W. B. Wherry, of this Bureau. Unfortunately Dr. Wherry left Manila before the work could be completed. Indications of nitrification were not lacking in his work, which is sufficiently encouraging to be continued. The assistance of nitrifying organisms would be a material advantage to the coconut, although it has been shown above that the amount of water which the tree takes up and transpires would, even in such poor soil as that encountered along the beach, contain a sufficient quantity of inorganic constituents to allow the plant to thrive.-P. C. F.

${ }^{29}$ It is true that in a wet soil the food is in more dilute solution than in a dry one, but this is partially compensated for by the selective absorption of nutrient salts from very dilute solutions, the solution absorbed being more concentrated than that in the ground. The more dilute the solution the greater is this selective power. 
as applying a fertilizer to the ground. 'The amount of transpiration can be increased in two ways-by increasing the amount of water at the disposal of the roots and by improving the conditions for its evaporation from the leaves.

In seasons. of drought the first method does the plant a double service, for the water which is artificially furnished is not only valuable in itself but also because of the substances dissolved in it. However, during other seasons, irrigation may not merely be useless but even very injurious, for ground too wet does not favor the activity of coconut roots any more than that which is too dry.

We have seen that the transpiration of the coconut is somewhat accelerated by the wind, and greatly so by intense illumination. 'Therefore, so long as the roots are not in too dry a soil, it is in the plant's interest to be exposed as much as is normally possible to these two agents. On any considerable tract devoted to coconut culture this can be done in but one way-by not planting the trees too close together. I have never seen a grove in which the trees were sufficiently far apart so that, unless other conditions were very unfavorable, the trees around the outside were not much more productive than those in the interior. At San Ramon, a considerable proportion of the trees are planted in double rows, one row along each side of a narrow road. In such a row, which contained no nonbearing trees, I found the yield at one cutting to average 22 nuts to the tree. A row of trees along the well-drained bank of a slough yielded an average of 27 nuts, all trees producing. A single tree standing by itself in the open yielded $\tilde{5} 5$ nuts. In the interior of an old grove, the average for the producing trees was about 11, and in the same situation in a large one on the neighboring hacienda of Talisayan the average for bearing trees was only 8 ; the individuals in the area where this count was made were as a rule about 18 feet apart, their crowns interlaced freely, producing a rather dense shade, and many trees were without ripe nuts.

I have no doubt that up to a distance of at least 15 meters any increase in the intervals between trees will result in an appreciable advance in the average yield per tree, but by planting beyond the intervals at which the interlacing of roots and of leaves would bring the trees into keen competition for water and light, and would also largely break the wind passing through the crowns, the increase in the yield of nuts for the individual trees would not be commensurate with the area of land in use. In my opinion, the trees in a grove can usually best be placed at intervals of about 9 meters. In exposed rows they may well be closer together, and where intense cultivation is economically possible the distance between them may be a little less.

The natural habitat of the coconut is the strand. It is restricted to this because it bears fruit too large to be practically transportable by any 
other natural agent than the water; and it is adapted thereto by possessing superficial roots which are uninjured by temporary exposure to concentrated solutions, hy having a tough, very elastic trunk, and by producing leaves which are not merely tolerant of the most intense insolation and wind but which are unable to work to the best purpose without more light and wind than many plants can endure. As is true for every cultivated plant, it is possible to create for the coconut conditions altogether more favorable for its utmost thrift than are ever known to occur in nature. It naturally grows in a "poor" soil-that is, in one in which its mineral and nitrogenous raw food is present in very dilute solution. We can improve its environment in this respect, and can profitably carry this improvement much further than is the general practice at present. But the coconut must not be expected to thrive, even in the richest soil and with the best cultivation, if its supply of light is restricted by other trees or in any other way, or where the air is too still or an adequate supply of water is not always available near the surface of the ground.

There is another method of increasing the yield of coconuts, slower but more permanent than improved cultivation; this is by the selection of seed. I have done nothing with this subject, and only mention it because the results of selection can not appear for many years, and a mistaken method would be long in showing its uselessness. Nuts obviously should be selected for seed from trees conspicuous for the amount or quality of their yield. It is usually not a difficult matter to decide whether or not the tree's superior yield is due to its growing under exceptionally favorable conditions. If it is, it shows how other trees may be made to bear equally well, but there is no reason for selecting the nuts of such a tree for seed; its offspring can not be expected to bear more nuts under ordinary conditions than the parent would have done without its exceptional advantages. The environment is not hereditary. The tree the nuts of which should be used as seed is the one the production of which is great in proportion to its opportunity. A tree bearing regularly 12 nuts to the cutting under conditions which allow its neighbors but 8 should have its nuts saved for seed in preference to those of an individual having 30 nuts among equally productive neighbors. 
Hygrometer resulings, Sam Romon frorm.

NOVEMBER, 1904.

\begin{tabular}{|c|c|c|c|c|c|c|c|c|c|}
\hline \multirow{3}{*}{ Date. } & \multicolumn{8}{|c|}{ Berch. } & \multirow{3}{*}{$\begin{array}{l}\text { Rain- } \\
\text { fall to } 4 \\
\text { p. m. }\end{array}$} \\
\hline & \multicolumn{3}{|c|}{6 a.m. } & \multicolumn{2}{|c|}{$12 \mathrm{~m}$} & \multicolumn{3}{|c|}{4 p. $\mathrm{m}}$. & \\
\hline & Dry. & Wet. & $\begin{array}{c}\text { Relative } \\
\text { humid- } \\
\text { ity. }\end{array}$ & Dry. $\mathbf{W e}$ & $\begin{array}{c}\text { Relative } \\
\text { humid- } \\
\text { ity. }\end{array}$ & \begin{tabular}{l|l} 
tive \\
aid- \\
Dry.
\end{tabular} & Wet. & $\begin{array}{c}\text { Relative } \\
\text { humid- } \\
\text { ity. }\end{array}$ & \\
\hline $15 \ldots$ & $\mid \begin{array}{c}0 \\
-\end{array}$ & 0 & Per cent. & \begin{tabular}{c|c}
0 & 0 \\
\end{tabular} & Per cent. & \begin{tabular}{c|c} 
cent. & 0 \\
& 25
\end{tabular} & $\begin{array}{l}\circ \\
23.6\end{array}$ & $\begin{array}{c}\text { Per cent. } \\
89\end{array}$ & $m_{27}^{m m}$ \\
\hline $166_{-}$ & 23.6 & 23.1 & 95 & \begin{tabular}{l|l}
24.1 & 23
\end{tabular} & 91 & \begin{tabular}{l|l}
1 & 23.1
\end{tabular} & 22.9 & 98 & 16 \\
\hline $17 \ldots$ & 23.9 & 22.1 & $85+$ & 25.5 & 85 & 24.9 & 23.8 & 91 & 3.5 \\
\hline $18 \ldots$ & 24.5 & 23.7 & 93 & 26 & 88 & 26.3 & 24.6 & 87 & 29 \\
\hline 19 & 24.2 & 23.8 & 97 & 27.5 & 95 & 26.2 & 24.2 & 85 & 7 \\
\hline 20 & 25 & 23.8 & $91-$ & 29.1 & 75 & 29.1 & 26.4 & 80 & Trace. \\
\hline 21 & 24.8 & 23.2 & $91-$ & 29.8 & 82 & 28.5 & 26.3 & 84 & Trace. \\
\hline 22 & 24.5 & 23.4 & 91 & 28.9 & 80 & 28.6 & $2 \overline{7} .7$ & 79 & Trace. \\
\hline 23 & 27.9 & 25.4 & 81 & 28.6 & 4 & 24.3 & 23.4 & $93-$ & 9 \\
\hline $24 \ldots$ & 24.5 & 23.5 & $91-$ & 29.7 & 7 & 26 & 24.5 & 88 & 0 \\
\hline $25 \ldots$ & 24.6 & 21.6 & 78 & 23.6 & 6 & 28 & 24.6 & 76 & 0 \\
\hline 26 & 25 & 23.4 & 87 & 29 & 3 & 29.6 & 24.1 & 63 & 0 \\
\hline 27 & 23 & 21.2 & 84 & 年 & & 27.5 & 24.2 & 76 & 0 \\
\hline $2 s \ldots$ & 21.2 & 20.2 & 91 & ------ & & $-\ldots$ & $-\ldots$ & $--\cdots$ & - \\
\hline 30 & $-\cdots--$ & $-\cdots-$ & 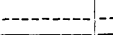 & $-\cdots--$ & & \begin{tabular}{l|l}
27.9 \\
-
\end{tabular} & 24.8 & 79 & 0 \\
\hline A verage .... & 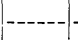 & $\mid-\cdots$ & 88.8 & $-\cdots--$ & & $\mid---$ & $-\cdots$ & 83.3 & 91.5 \\
\hline & & & & & Interi & & & & \\
\hline Date. & & 6 a. $\mathrm{m}$ & & & $12 \mathrm{~m}$. & & & 4 p. $\mathrm{m}$. & \\
\hline & Dry. & Wet. & $\begin{array}{c}\text { Relative } \\
\text { humid- } \\
\text { ity. }\end{array}$ & Dry. & Wet. & $\begin{array}{c}\text { Relative } \\
\text { humid- } \\
\text { ity. }\end{array}$ & Dry. & Wet. & $\begin{array}{l}\text { Relative } \\
\text { humid- } \\
\text { ity. }\end{array}$ \\
\hline 15 & $\begin{array}{c}0 \\
-\end{array}$ & $0^{\circ}$ & Per cent. & $\begin{array}{c}0 \\
-0\end{array}$ & $\begin{array}{c}0 \\
-\end{array}$ & Per cent. & $\begin{array}{l}\circ \\
24.5\end{array}$ & $\begin{array}{l}\circ \\
23.3\end{array}$ & $\begin{array}{c}\text { Per cent } \\
90+\end{array}$ \\
\hline 16 & 23 & 22.5 & $95+$ & 23.2 & 22.9 & 98 & 23 & 22.9 & 99 \\
\hline 17 - & 23.9 & 22.5 & 87 & 25.3 & 23.1 & 83 & 24.5 & 23.3 & $91-$ \\
\hline 18 & 23.5 & 23.2 & 98 & 24.5 & 23.5 & 92 & 25 & 24.2 & 93 \\
\hline 19 & 24 & 23.5 & 96 & 28.5 & 25 & 75 & 25.5 & 24.5 & 91 \\
\hline 20 _nn & 27.5 & 23.4 & $91-$ & 29.4 & 25.5 & 73 & 28 & 25.5 & 82 \\
\hline 21 - & 24.3 & 23 & 89 & 28.4 & 25.7 & 80 & 27.5 & 25.2 & 83 \\
\hline 22 - & 22.5 & 22 & 95 & 28.3 & 25.7 & 80 & 28.4 & 25.6 & 80 \\
\hline 23 & 26.9 & 24.5 & 81 & 29 & 26 & 78 & 23.5 & 22.8 & 94 \\
\hline 24 & 24.5 & 23.4 & 91 & 28.9 & 25.4 & 76 & 25.9 & 24.5 & 89 \\
\hline 25 & 23.9 & 22.6 & 89 & 29 & 24.5 & $69+$ & 26.2 & 24.4 & 85 \\
\hline 26 & 23.8 & 23 & 93 & 28.5 & 24 & 69 & 28 & 23.5 & 69 \\
\hline 27 - $27-1$ & 21.8 & 20.8 & 91 & 29.4 & 25 & - & 27.2 & 24.5 & 80 \\
\hline 28 & 21 & 20.2 & 93 & --n-- & $-\cdots-1-1$ & $-\cdots$ & ----- & ---- & ---- \\
\hline 30 & --- & & & $-\cdots-$ & & $-\cdots-1$ & 27.8 & 25.2 & 81 \\
\hline Average --. & & & 91.5 & ---- & ----- & 81.2 & --- & $-\cdots$ & 87.6 \\
\hline
\end{tabular}


H!ygrometer readings, sim Ramon furm-Continued.

NOVEMBER, 1904-Continued.

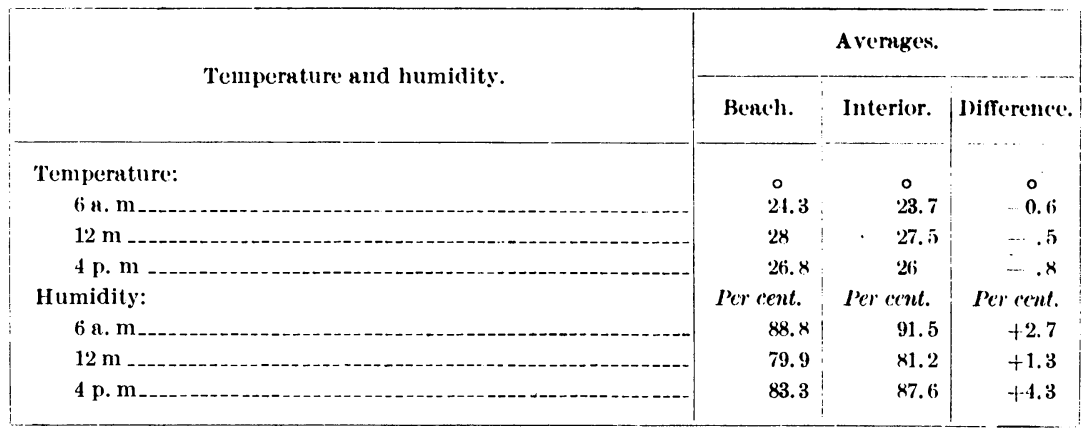

DECEMBER, 1904.

\begin{tabular}{|c|c|c|c|c|c|c|c|c|c|}
\hline \multirow{3}{*}{ Dute. } & \multicolumn{8}{|c|}{ Beach. } & \\
\hline & \multicolumn{3}{|c|}{7.30 a. $\mathrm{m}$. } & \multicolumn{3}{|c|}{11.30 a. m. } & \multicolumn{3}{|c|}{4 p. m. } \\
\hline & Dry. & Wet. & $\begin{array}{l}\text { Relative } \\
\text { humid- } \\
\text { ity. }\end{array}$ & Dry. & Wet. & $\begin{array}{c}\text { Relative } \\
\text { humid- } \\
\text { ity. }\end{array}$ & Dry. & Wet. & $\begin{array}{c}\text { Relative } \\
\text { humid- } \\
\text { ity. }\end{array}$ \\
\hline $1 \ldots$ & $\begin{array}{l}0 \\
22.6\end{array}$ & $\begin{array}{c}\circ \\
21.6\end{array}$ & $\underset{92}{\text { Per cent. }}$ & $\begin{array}{l}\circ \\
26.4\end{array}$ & $\begin{array}{l}\circ \\
24.5\end{array}$ & $\begin{array}{c}\text { Per cent. } \\
85\end{array}$ & $\begin{array}{l}\circ \\
27.9\end{array}$ & $\begin{array}{l}\circ \\
24.6\end{array}$ & $\underset{76}{\text { Per cent. }}$ \\
\hline 2 - $2-1-1-0-1$ & 25 & 22.3 & 79 & 29 & 24 & 66 & 26.2 & 23.9 & 83 \\
\hline 3 - & 27.2 & 23.6 & 74 & 28.5 & 24 & 69 & 27.6 & 23.5 & 72 \\
\hline $4 \ldots$ & 24.3 & 22.7 & 87 & ----- & - & ---- & 28 & 24.2 & 73 \\
\hline 5 & 25.5 & 22.6 & 78 & 28.8 & 23.7 & $65+$ & 29.1 & 24.5 & $6 x$ \\
\hline $6 \ldots$ & 27.4 & 24.1 & 76 & 27 & 24.3 & 80 & 28 & 25.1 & 79 \\
\hline 7 ---- & 26.4 & 24.4 & 85 & 28.3 & 25.5 & 80 & 27.2 & 25 & 84 \\
\hline $8-\ldots$ & 25.1 & 23.2 & 85 & 28 & 26 & 86 & 28.3 & 24.8 & 75 \\
\hline 9 & 25.3 & 24.1 & 91 & 28.9 & 25.9 & 78 & 27.8 & 25.5 & 83 \\
\hline 10 - & 26.9 & 24.5 & 81 & 28.5 & 26.3 & 84 & 27.6 & 25.5 & 85 \\
\hline 11 & ----- & ------ & 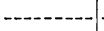 & ------ & $---\cdots$ & - - - & 27.3 & 25.3 & 85 \\
\hline $12 \ldots$ & 26.2 & 24 & 83 & 29 & 26 & 79 & 28.1 & 25.5 & 81 \\
\hline 13 & 26.4 & 23.7 & 79 & 27.7 & 24.8 & 79 & 28.5 & 25.5 & 78 \\
\hline 14 & 26.2 & 24.3 & 85 & $-\ldots$ & ----- & ------ & ---- & $---\cdots$ & --- \\
\hline 15 _ & 26.8 & 25.7 & 92 & 27.7 & 25.5 & 84 & 28 & 25.6 & 83 \\
\hline 16 & 26.5 & 24.7 & 86 & 28.1 & 26.1 & 82 & 29.5 & 27.2 & 84 \\
\hline $17 \ldots$ & 25.7 & 23.9 & 86 & 29.1 & 25.3 & 73 & 28.9 & 25.5 & 76 \\
\hline 18 & 26.5 & 23.9 & 81 & ----- & $-\ldots$ & $-\cdots$ & 28.2 & 26.3 & 87 \\
\hline 19 -- & 25 & 23.6 & 89 & 28.6 & 25.3 & 77 & 27.5 & 24.8 & 80 \\
\hline 20 ---- & 25.5 & 24.4 & 91 & 28.3 & 25.2 & 78 & 26.3 & 24 & 83 \\
\hline $21 \ldots$ & 24 & 22.7 & 89 & 27.5 & 25.4 & 85 & 27.2 & 25 & 84 \\
\hline $22--$ & 25.3 & 23.7 & 87 & 27.5 & 24.2 & 76 & 27.7 & 25 & 78 \\
\hline $23-\ldots$ & 25.9 & 23.7 & 83 & 28 & 25.5 & 82 & 28.5 & 25.9 & 81 \\
\hline $24 \ldots$ & 25.7 & 24 & 87 & 27.1 & 24.8 & 83 & 26.6 & 24.6 & 85 \\
\hline 25 & 25.6 & 24.2 & 89 & 27.7 & 24.8 & 79 & 26.9 & 24.7 & 83 \\
\hline $266^{\circ}$ & 24.4 & $2 \cdot 2$ & 80 & -------- & ----- & 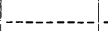 & ----- & ---- & $\cdots$ \\
\hline 27 - & 26.3 & 24.6 & 87 & 27.4 & 25.3 & 85 & 26.8 & 25.4 & 89 \\
\hline 28 & 26.7 & 24.3 & 82 & 27.3 & 25.1 & 84 & 27.6 & 25 & 81 \\
\hline 29 - & 25 & 22.5 & 80 & ------ & ---- & ------ & -n- & ---- & \\
\hline 30 - & 26.5 & 24 & 81 & 28.5 & 25.1 & 76 & 28.1 & 25 & 78 \\
\hline 31 - & 26.2 & 24 & 83 & 27.2 & 25.2 & 85 & - & & - \\
\hline A verage & $-\cdots$ & 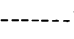 & 84.27 & & ----- & 79.2 & ---n- & $-\cdots$ & 80.52 \\
\hline
\end{tabular}


Hygrometer readings, San Ramon farm-Continued.

DECEM BER, 1904-Continued.

\begin{tabular}{|c|c|c|c|c|c|c|c|c|c|c|}
\hline \multirow{3}{*}{ Date. } & \multicolumn{9}{|c|}{ Interior. } & \multirow{3}{*}{$\begin{array}{l}\text { Rain- } \\
\text { fall. }\end{array}$} \\
\hline & \multicolumn{3}{|c|}{7.30 a. m. } & \multicolumn{3}{|c|}{$11.30 \mathrm{a} . \mathrm{m}$. } & \multicolumn{3}{|c|}{4 p. m. } & \\
\hline & Dry. & Wet. & $\begin{array}{c}\text { Relative } \\
\text { humid- } \\
\text { ity. }\end{array}$ & Dry. & Wet. & $\begin{array}{c}\text { Relative } \\
\text { humid- } \\
\text { ity. }\end{array}$ & Dry. & Wet. & $\begin{array}{c}\text { Relative } \\
\text { humid- } \\
\text { ity. }\end{array}$ & \\
\hline 1 & $\begin{array}{l}\circ \\
22.5\end{array}$ & $\begin{array}{l}\circ \\
21.9\end{array}$ & $\begin{array}{r}\text { Percent. } \\
95\end{array}$ & $\begin{array}{l}\circ \\
26.5\end{array}$ & $\begin{array}{l}\circ \\
24.6\end{array}$ & $\begin{array}{c}\text { Per cent. } \\
85\end{array}$ & $\begin{array}{l}\circ \\
26.4\end{array}$ & $\begin{array}{c}\circ \\
25\end{array}$ & $\begin{array}{c}\text { Per cent. } \\
89\end{array}$ & $\begin{array}{r}m m . \\
\text { Trace. }\end{array}$ \\
\hline 2 - & 25 & 22.8 & 83 & 28.7 & 25.4 & 77 & 26.5 & 24. 7 & 86 & 0 \\
\hline 3. & 28.3 & 25.2 & 77 & 28.7 & 25.6 & 78 & 27 & 23.5 & 74 & 0 \\
\hline 4.---------- & 24.3 & 23 & 89 & $-\ldots$ & --- & 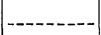 & 27.2 & 24.2 & 78 & 0 \\
\hline 5 & 25.5 & 22.5 & 77 & 28 & 24 & 72 & 28.1 & 24.1 & 72 & 0 \\
\hline 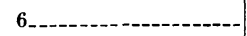 & 28 & 24.4 & 74 & 27.9 & 25 & 79 & 27.2 & 23.9 & 83 & Trace. \\
\hline 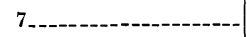 & 26.9 & 24.5 & 82 & 28 & 25.8 & 84 & 26.8 & 25 & 86 & Trace. \\
\hline 8-1 & 25.9 & 24.2 & 87 & 28.6 & 26.1 & 82 & 27.5 & 25.1 & 83 & 0 \\
\hline 9 & 24.9 & 24 & 93 & 30.6 & 27.5 & 78 & 27.5 & 25.7 & 87 & Truce. \\
\hline 10 & 26.3 & 24.5 & 86 & 27.9 & 25 & 86 & 27.5 & 25 & 82 & Trace. \\
\hline 11 & ----- & ---- & ------- & --- & ---- & $\mid-----\infty$ & 27 & 25 & 85 & 0 \\
\hline $12 \ldots$ & 26.1 & 24 & 84 & 27.9 & 25 & 79 & 27.6 & 25.4 & 84 & 0 \\
\hline 13 & 26.3 & 24 & 83 & 28 & 24.3 & 74 & 27.9 & 24.9 & 78 & 0 \\
\hline 14 & 26.5 & 24.6 & 85 & ----- & ---- & ----- & $-\cdots$ & ---- & ! & 0 \\
\hline 15 & 25.4 & 23.5 & 85 & 27.6 & 25.4 & 84 & 27.5 & 25 & 82 & 1 \\
\hline 16 & 28 & 25.6 & 83 & 27.6 & 25 & 81 & 28.3 & 25.7 & 81 & Truce. \\
\hline $17 \ldots$ & 25.3 & 23.9 & 89 & 27.8 & 24.5 & 76 & 28.1 & 25 & 78 & 0 \\
\hline 18 & 26.6 & 24.2 & 81 & ----- & $-\cdots$ & $\mid-\ldots-n$ & 27.6 & 25.5 & 87 & Trace. \\
\hline 19 & 25.8 & 24.2 & 87 & 27.6 & 25 & 81 & 27.5 & 24.3 & 77 & Truce. \\
\hline $20 \ldots$ & 25.5 & 24.6 & 93 & 28.5 & 25.6 & 79 & 26.2 & 24.3 & 85 & 1 \\
\hline $21 \ldots$ & 23.7 & 23 & 94 & 28.4 & 25.4 & 78 & 27.3 & 24.8 & 82 & Trace. \\
\hline $22 \ldots$ & 24.8 & 23.3 & 88 & 27.6 & 24.2 & 76 & 27.4 & 24.7 & 80 & Trace. \\
\hline $23_{-}$ & 26.5 & 24 & 81 & 27.5 & 25.2 & 84 & 27.4 & 24.8 & 81 & 0 \\
\hline 24 & 25.1 & 23.3 & 86 & 27.2 & 24.2 & 78 & 26.2 & 24.4 & 86 & 0.5 \\
\hline $25 \ldots$ & 25.1 & 24 & 91 & 27.5 & 24.5 & 78 & 26.5 & 24.5 & 85 & 0 \\
\hline 26 & 24.1 & 23.1 & 91 & ---- & --- & $-\ldots$ & $-\ldots$ & $-\ldots$ & -1 & 0 \\
\hline 27_----_---- & 26.8 & 25 & 86 & 27.2 & 25.1 & 85 & 26.7 & 24.6 & 84 & 0 \\
\hline 28 & 26.8 & 24.5 & 83 & 27.9 & 25.3 & 81 & 27.6 & 24.4 & 77 & 0 \\
\hline 29 & 25.5 & 22.9 & 80 & ---- & ---- & - & - & 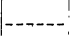 & - & 0 \\
\hline 30 & 27.7 & 24.1 & 74 & 29.6 & 25 & 69 & 27.5 & 24.5 & 78 & 0 \\
\hline 31_..... & 27.1 & 24.9 & 83 & 28.5 & 25.5 & 78 & ---- & $-\cdots-$ & - & 0 \\
\hline Average & & ---- & 85 & --- & $-\cdots$ & 79.28 & & ---- & 81.85 & 2.5 \\
\hline
\end{tabular}


H!ygrometer readings, Sim Kamon furm-Continued.

JANUARY, 1905.

\begin{tabular}{|c|c|c|c|c|c|c|c|c|c|}
\hline \multirow{3}{*}{ Date. } & \multicolumn{9}{|c|}{ Beuch. } \\
\hline & \multicolumn{3}{|c|}{7.30 a. $\mathrm{m}$. } & \multicolumn{3}{|c|}{$11.30 \mathrm{a} . \mathrm{m}}$. & \multicolumn{3}{|c|}{4 p. m. } \\
\hline & Dry. & Wet. & $\begin{array}{l}\text { Relative } \\
\text { humid- } \\
\text { ity. }\end{array}$ & I)ry. & Wet. & $\begin{array}{c}\text { Relative } \\
\text { humid- } \\
\text { ity. }\end{array}$ & Dry. & wet. & $\begin{array}{l}\text { Relutive } \\
\text { humid- } \\
\text { ity. }\end{array}$ \\
\hline & 0 & $\circ$ & Per cent. & $\circ$ & $\circ$ & Per cent. & $\circ$ & 0 & Per cent. \\
\hline 2. & $\ldots$ & -1 & & $\ldots$ & $-\ldots$ & .... & $\ldots$ & $\ldots$ & - \\
\hline $3 \ldots$ & 26 & 24.1 & 85 & 29.4 & 24.2 & 65 & 27.5 & 24.5 & 78 \\
\hline $4 \ldots$ & 26 & 24.3 & 87 & 28.6 & 24.5 & 71 & 26.6 & 24 & 80 \\
\hline $5 \ldots$ & 24.8 & 23 & 86 & 27.5 & 23.9 & 74 & 27.1 & 24 & 77 \\
\hline 6 & 27.6 & 24.2 & 76 & 27.5 & 25 & 82 & 27.1 & 24.8 & $\times 3$ \\
\hline 7 -.-- & 25.7 & 23.2 & 81 & 28 & 23.6 & 69 & 27.5 & 23.8 & 73 \\
\hline $8 \ldots$ & 26 & 24.4 & 87 & ------- & - & - & 27 & 24.6 & 82 \\
\hline 9 & 24.7 & 23 & 87 & 28.3 & 24.6 & 74 & 26.5 & 24.4 & 84 \\
\hline - & 25.5 & 23.7 & 86 & 27.5 & 24.3 & 77 & 27.5 & 25 & 82 \\
\hline 11 & 25.4 & 23.8 & 87 & 28.3 & 25.1 & 77 & 26.5 & 24.5 & 85 \\
\hline $12 \ldots$ & 26.8 & 24 & 79 & 29.7 & 26 & 74 & 26.3 & 24.5 & 86 \\
\hline 13 - & 22.9 & 22.5 & 97 & 27.6 & 24.9 & 80 & 27.3 & 25.1 & 84 \\
\hline $14 \ldots$ & -.--- & $-\ldots$ & ----- & --n--- & --- & $\ldots$ & - - - - - & 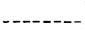 & --- \\
\hline 15 & -.--- & ----- & - & ----- & ---- & - & - & - - - - & - \\
\hline $16 \ldots$ & $-\ldots$ & $\mid-\ldots$ & $\ldots$ & 29.1 & 24.6 & 70 & 27 & 24.5 & 82 \\
\hline $17 \ldots$ & 24.4 & 21.9 & 80 & 27.6 & 24.6 & 78 & 27.8 & 24.2 & 74 \\
\hline 18 -.-- & 24.1 & 22.5 & 87 & 27.4 & 24 & 75 & 28 & 24.5 & 75 \\
\hline 19 & 25.1 & 23.4 & 87 & 28.8 & 24 & 67 & 28 & 24.7 & 76 \\
\hline $20 \ldots$ & 24.3 & 23.1 & 91 & 28 & 25.5 & 82 & 28.8 & 25.3 & 75 \\
\hline $21 \ldots$ & 25.4 & 23.3 & 84 & 28 & 25.8 & 84 & 27 & 24.8 & 81 \\
\hline $22 \ldots$ & 27.6 & 24.8 & 80 & - & --- & ---- & 27.5 & 24.7 & 80 \\
\hline $23 \ldots$ & $-\ldots$ & ----- & ------ & 28.8 & 25.9 & 79 & 27.2 & 25.2 & 85 \\
\hline $24 \ldots$ & 25.1 & 23.5 & 87 & 27.5 & 25 & 82 & 27.6 & 25.3 & 84 \\
\hline $25 \ldots$ & 26 & 24.1 & 85 & 28 & 25.3 & 80 & 28.5 & 25.8 & 80 \\
\hline $26 \ldots$ & 25.2 & 23.4 & 86 & 28.7 & 25.9 & 80 & 26.8 & 24.8 & 85 \\
\hline $27 \ldots$ & 24.5 & 21.8 & 79 & 28.9 & 25.1 & 73 & 27.8 & 25.1 & 80 \\
\hline $28 \ldots$ & 24.5 & 22.5 & 85 & 29.4 & 25.8 & 75 & 28.9 & 25.6 & 77 \\
\hline 29 & & & & & & & & & \\
\hline $30 \ldots$ & 25 & 22.5 & 80 & $---1-$ & - & - & ------ & --_- - - &.---- \\
\hline 31 & $-\cdots-$ & - & - & 28 & 24 & 72 & 28.2 & 24.8 & 76 \\
\hline Average & & - & 84.74 & --- & - & 75.65 & -------- & ----- & 80.28 \\
\hline
\end{tabular}


Hygrometer readings, San Ramon farm-Continued.

JANUARY, 1905-Continued.

\begin{tabular}{|c|c|c|c|c|c|c|c|c|c|c|}
\hline \multirow{3}{*}{ Iate. } & \multicolumn{10}{|c|}{ Interior. } \\
\hline & \multicolumn{3}{|c|}{7.30 a. m. } & \multicolumn{3}{|c|}{11.30 a. m. } & \multicolumn{3}{|c|}{4 p. m. } & \multirow{2}{*}{$\begin{array}{c}\text { Ruin- } \\
\text { fall. }\end{array}$} \\
\hline & Iry. & Wet. & $\begin{array}{c}\text { Relative } \\
\text { humid- } \\
\text { ity. }\end{array}$ & Dry. & Wet. & $\begin{array}{l}\text { Relutive } \\
\text { humid- } \\
\text { ity. }\end{array}$ & Dry. & Wet. & $\begin{array}{c}\text { Relative } \\
\text { humid- } \\
\text { ity. }\end{array}$ & \\
\hline 1. & $\circ$ & 0 & Per cent. & $\circ$ & 0 & Per cent. & 0 & 0 & Per cent. & $m m$. \\
\hline $2 \ldots$ & ---- & -- - - & $-\ldots$ & $\ldots$ & --- & - & - & -.--- & ----- --- & 0 \\
\hline 3. & 26.9 & 24.4 & 81 & 29 & 24 & 66 & 27.3 & 24.1 & 77 & Trace. \\
\hline $4 \ldots$ & 26.5 & 23.9 & 81 & 29 & 24.2 & 68 & 28 & 24.7 & 76 & 0 \\
\hline 5 & 26 & 23.5 & 81 & 28 & 23.6 & 69 & 26.5 & 23.6 & 78 & 0 \\
\hline 6 & 27.9 & 24.5 & 76 & 28.2 & 25 & 77 & 26.5 & 24.4 & 84 & 0 \\
\hline 7 & 26.5 & 23.7 & 79 & 28.5 & 23.8 & 68 & 27.2 & 23.6 & 74 & 0 \\
\hline 8 & 26.2 & 24.5 & 87 & ---- & ---- & 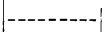 & 26.6 & 24.2 & 82 & 0 \\
\hline $9 \ldots$ & 25.2 & 23.6 & 87 & 28 & 24 & 72 & 26.5 & 24.3 & 83 & 0 \\
\hline 10 & 25.5 & 23.6 & 85 & 28.5 & 25.4 & 78 & 27.5 & 24.8 & 80 & 0 \\
\hline $11-\ldots$ & 25.9 & 24.2 & 87 & 27.7 & 24.7 & 78 & 26.1 & 24.3 & 86 & 0 \\
\hline 12 & 27.5 & 24.6 & 79 & 29.4 & 25.7 & 74 & 26.5 & 24.3 & 87 & 2 \\
\hline 13 & 23.1 & 22.8 & 98 & 27 & 24.4 & 81 & 26.9 & 24.7 & 83 & 26 \\
\hline 14 & ---- & $-\cdots---$ & ---- & ---- & ---- & -.-- - - & ----- & ---- & ------ & 0 \\
\hline 15 & --- & ---- & 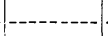 & --- & --- & 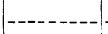 & --- & ---- & -...- & 0 \\
\hline $16 \ldots$ & ---- & ---- & $---\infty$ & 29.4 & 25.4 & 72 & 26.5 & 24.5 & 85 & 0 \\
\hline $17---$ & 25 & 22.6 & 81 & 27.5 & 24.6 & 79 & 27.3 & 24.1 & 77 & 0 \\
\hline 18 & 24.4 & 22.9 & 88 & 27.5 & 24 & 75 & 27.3 & 24.2 & 78 & 0 \\
\hline 19 & 24.7 & 23.2 & 88 & 28.8 & 23. 7 & 68 & 27.3 & 24.3 & 78 & 0 \\
\hline $20 \ldots$ & 24.3 & 23.3 & 92 & 28.5 & 25.5 & 78 & 28 & 25 & 78 & 0 \\
\hline $21 \ldots$ & 25 & 23.3 & 87 & 29 & 26.3 & 80 & 27 & 25 & 85 & 0 \\
\hline $22 \ldots$ & 27.7 & 24.7 & 78 & $\ldots$ & ------ & - & 27.5 & 25.5 & 86 & 2 \\
\hline $23 \ldots$ & ----- & ---- & --- & 27.4 & 24.6 & 80 & 27.1 & 25.1 & 85 & 0 \\
\hline 24 & 24.9 & 23.5 & 89 & 28 & 25 & 78 & 28 & 25.4 & 81 & 0 \\
\hline $25 \ldots$ & 26.4 & 24.4 & 85 & 28.5 & 25.5 & 79 & 27.7 & 25 & 80 & Trace. \\
\hline $26 \ldots$ & 25.6 & 24 & 87 & 28.6 & 25.6 & 79 & 27 & 25.2 & 80 & 0 \\
\hline 27 & 24.7 & 22 & 79 & 28.7 & 24.9 & 73 & 27.5 & 24.5 & 78 & 0 \\
\hline $28 \ldots$ & 25.6 & 22.5 & 77 & 29 & 25.4 & 75 & 28.3 & 25.2 & 78 & 0 \\
\hline 29 & --- & --- & & & $-\cdots-$ & & & & & \\
\hline 30 & 26.3 & 24 & 83 & & & & ---- & $-\cdots$ & --------- & 0 \\
\hline 31 & $-\cdots--$ & --.-- & 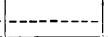 & 28 & 23.7 & 70 & 27.6 & 24.3 & 76 & 0 \\
\hline Average --- & ------ & ---- & 84.13 & 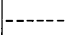 & ---- & 74.65 & ---- & ----- & 80.60 & 30 \\
\hline
\end{tabular}


H!ygromele'r madings, Sam Ramom farm-Continued.

FEBRUARY, 1905.

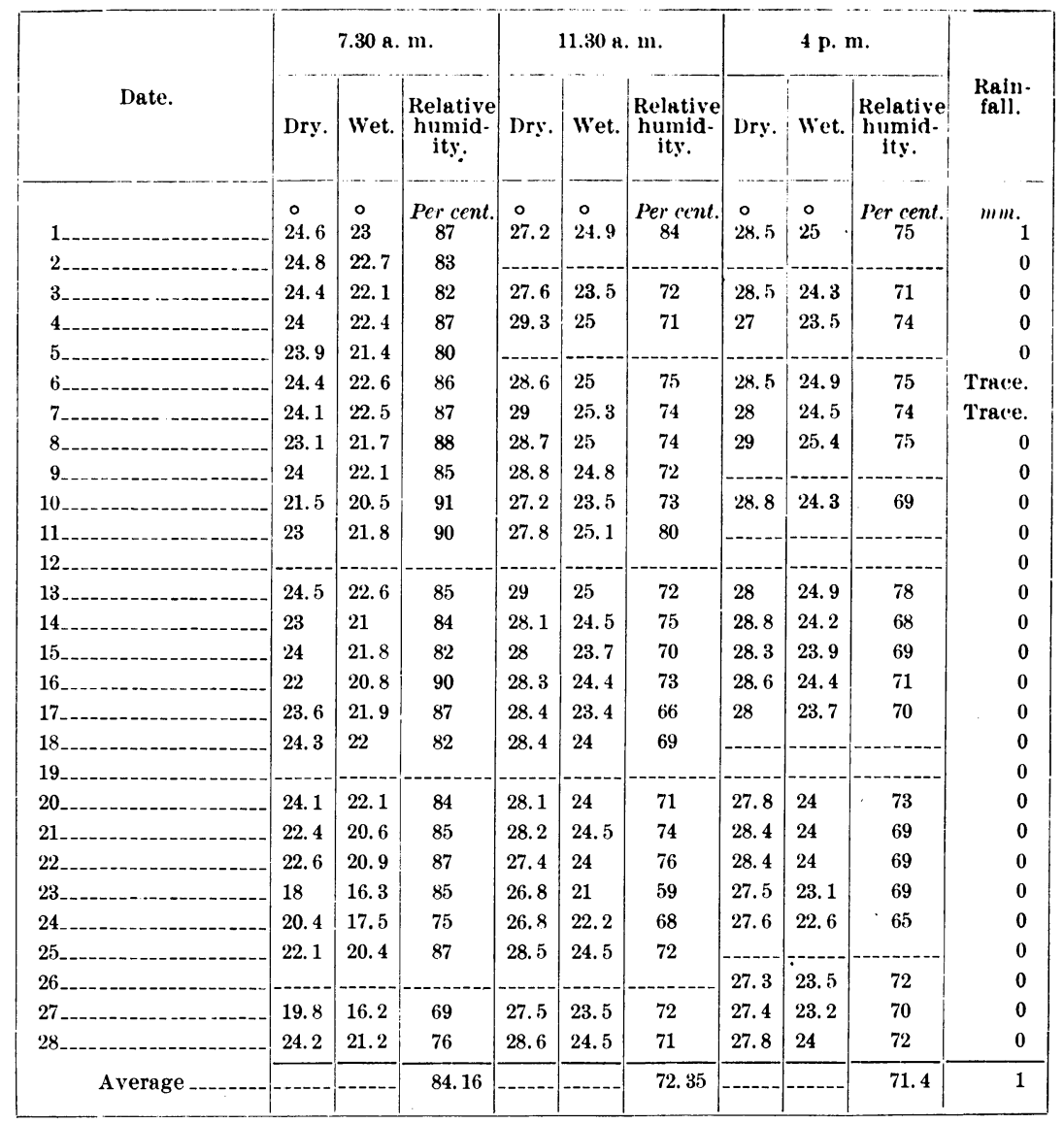

$36540-4$ 
Hygrometer readings, San Ramon farm-Continued.

MARCH, 1905.

\begin{tabular}{|c|c|c|c|c|c|c|c|c|c|c|}
\hline \multirow[b]{2}{*}{ Date. } & \multicolumn{3}{|c|}{7.30 a. $\mathrm{m}$. } & \multicolumn{3}{|c|}{11.30 a. m. } & \multicolumn{3}{|c|}{4 p. m. } & \multirow[b]{2}{*}{$\begin{array}{l}\text { Rain } \\
\text { fall. }\end{array}$} \\
\hline & Dry. & Wet. & $\begin{array}{l}\text { Relative } \\
\text { humid- } \\
\text { ity. }\end{array}$ & Dry. & Wet. & $\begin{array}{l}\text { Relative } \\
\text { humid- } \\
\text { ity. }\end{array}$ & Dry. & Wet. & $\begin{array}{c}\text { Relative } \\
\text { humid- } \\
\text { ity. }\end{array}$ & \\
\hline $1 \ldots$ & $\begin{array}{l}\circ \\
22.4\end{array}$ & $\begin{array}{c}\circ \\
20.4\end{array}$ & $\begin{array}{c}\text { Per cent. } \\
84\end{array}$ & $\begin{array}{l}\circ \\
28.3\end{array}$ & $\begin{array}{c}\circ \\
24\end{array}$ & $\begin{array}{c}\text { Per cent. } \\
70\end{array}$ & $\begin{array}{c}\circ \\
28\end{array}$ & $\begin{array}{l}\circ \\
24.5\end{array}$ & $\begin{array}{c}\text { Per rent. } \\
75\end{array}$ & $m m \cdot 0$ \\
\hline $2 \ldots$ & 22 & 19.5 & 79 & 27.8 & 23.5 & 70 & 28.5 & 25 & 75 & 0 \\
\hline $3_{-}$ & 24.3 & 22 & 82 & 28.4 & 24.6 & 73 & 27.5 & 24.1 & 75 & $\mathbf{0}$ \\
\hline $4 \ldots$ & 23.1 & 21 & 83 & 28 & 24.6 & 76 & ---- & ---- & ---- & 0 \\
\hline $5 \ldots$ & - & ---- & $-\ldots-\infty-1$ & $-\ldots-$ & $\ldots$ & $\ldots$ & -.- & $-\ldots$ & $\ldots$ & 0 \\
\hline $6 \ldots$ & 23.5 & 20.6 & 77 & ---- & $-\ldots$ & --- & 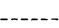 & ---- & --- & 0 \\
\hline $7 \ldots$ & ----- & ------ & --n-- & 29.1 & 24.6 & 69 & 27.5 & 24.3 & 77 & 0 \\
\hline $8 \ldots$ & 23.6 & 22 & 87 & 29 & 24.9 & 72 & 26.9 & 24.4 & 81 & 0 \\
\hline$-1-1-1-1-1$ & 24.8 & 23 & 86 & 28.2 & 23.6 & 68 & 26.8 & 23.5 & 75 & 0 \\
\hline $10 \ldots$ & 24 & 21.2 & 78 & 29 & 23.6 & 63 & 27.9 & 24 & 72 & 0 \\
\hline $11 \ldots$ & 23.2 & 21.2 & 84 & 28.7 & 24.2 & 69 & 27.5 & 23.5 & 71 & 0 \\
\hline $12 \ldots$ & 24.5 & 21.7 & 78 & 28.2 & 24.6 & 74 & 27.4 & 24.6 & 80 & 0 \\
\hline $13 \ldots$ & 24 & 22.1 & 85 & 28.5 & 25.5 & 78 & $\cdots--1$ & & $\ldots$ & 0 \\
\hline 14 & 25.2 & 23.3 & 85 & 28.6 & 26 & 81 & 28.9 & 25.5 & 76 & 0 \\
\hline $15 \ldots$ & 25 & 22 & 77 & 29 & 25.4 & 75 & 28.6 & 25.5 & 78 & 0 \\
\hline 16 & 25 & 23 & 84 & 29.1 & 25.8 & 77 & 28.7 & 25 & 74 & 0 \\
\hline 17 & 25.5 & 23.5 & 84 & 29 & 26 & 79 & 28 & 25.4 & 81 & Trace. \\
\hline - & 24.7 & 22.9 & 86 & 28.6 & 26 & 81 & -- & - & -- & 0 \\
\hline - & 25.8 & 23.8 & 85 & 29.8 & 26.3 & 76 & 29 & 26.1 & 79 & 0 \\
\hline $20 \ldots$ & 26.1 & 23.8 & 83 & 29.5 & 26.4 & 80 & 27.9 & 25.7 & 84 & 0 \\
\hline $21 \ldots$ & 24.5 & 22.5 & 84 & 29.5 & 26.5 & 79 & 28.9 & 26.1 & 80 & $\mathbf{0}$ \\
\hline $22 \ldots$ & 26 & 23.4 & 80 & 29.5 & 27 & 82 & 29.2 & 25.9 & 77 & $\mathbf{0}$ \\
\hline - & 26.5 & 23.9 & 80 & 29.1 & 26.3 & 80 & ----- & $-1-2=$ & $\ldots$ & $\mathbf{0}$ \\
\hline----------- & 25 & 22.8 & 83 & 29.3 & $26.5)$ & 80 & 29.8 & 26.4 & 77 & $\mathbf{0}$ \\
\hline $2-1-1-1-1-1$ & 26.2 & 23.3 & 78 & 29 & 25.9 & 78 & 28.3 & 25.8 & 82 & $\mathbf{0}$ \\
\hline 26 & ---- & $-\infty-\infty$ & 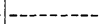 & ---- & --- & 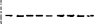 & ----- & & & 0 \\
\hline - non & 26.7 & 24 & 79 & 29.7 & 27.1 & 81 & 29.7 & 26.5 & 78 & 0 \\
\hline$-2-1-1-1$ & 27 & 24 & 78 & 29.6 & 26 & 75 & ---- & ---- & ---- & $\mathbf{0}$ \\
\hline $29_{---}$ & 26.2 & 23.6 & 81 & 31 & 27 & 73 & 29.8 & 26.3 & 77 & 0 \\
\hline - - - - - & 27.4 & 24.6 & 80 & 29.3 & 26 & 77 & 29.8 & 26.1 & 76 & Trace. \\
\hline $31 \ldots$ & 26 & 24.1 & 85 & 29.6 & 26.3 & 77 & 30 & 26.8 & 78 & 0 \\
\hline Average & -..... & ----- & 81.96 & & $\ldots$ & 75.46 & & & 77.30 & $\mathbf{0}$ \\
\hline
\end{tabular}


Hygrometer readings, Sim Ramon furm-Continued.

APRIL, 1905.

\begin{tabular}{|c|c|c|c|c|c|c|c|c|c|c|}
\hline & \multicolumn{3}{|c|}{7.30 a. m. } & \multicolumn{3}{|c|}{11.30 и. m. } & \multicolumn{3}{|c|}{4 p. m. } & \multirow[b]{2}{*}{$\begin{array}{c}\text { RHin- } \\
\text { fall. }\end{array}$} \\
\hline Date. & l)ry. & Wet. & $\begin{array}{l}\text { Relative } \\
\text { humid- } \\
\text { ity. }\end{array}$ & Dry. & Wet. & $\begin{array}{l}\text { Relative } \\
\text { humid- } \\
\text { ity. }\end{array}$ & Dry. & Wet. & $\begin{array}{c}\text { Relutive } \\
\text { humid- } \\
\text { ity. }\end{array}$ & \\
\hline 1 & $\begin{array}{c}0 \\
26.4\end{array}$ & $\begin{array}{l}0 \\
24.9\end{array}$ & Percent. & $\begin{array}{c}0 \\
28.1\end{array}$ & $\begin{array}{l}0 \\
25.9\end{array}$ & $\begin{array}{c}\text { Percent. } \\
84\end{array}$ & $\circ$ & 0 & Percent. & mum. \\
\hline $2 \ldots$ & 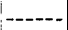 & $-\cdots$ & $-\cdots$ & --- & $-\cdots$ & -1 & -- & -- & 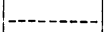 & 0 \\
\hline 3 & $-\cdots$ & ---- & - & 28.8 & 26.2 & 81 & 29 & 26 & 79 & Truce. \\
\hline 4 & 27.1 & 24.5 & 81 & 31 & 27.6 & 77 & 30 & 25.9 & 72 & 0 \\
\hline 5 & 26.9 & 24.1 & 79 & 30.5 & 25.3 & 66 & 29.3 & 25 & 70 & 0 \\
\hline $6 \ldots$ & 25.6 & 23.8 & 86 & 30.6 & 26.2 & 70 & 28.9 & 25.5 & 76 & 0 \\
\hline 7_-_- & 26.6 & 24.5 & 84 & 30 & 25.9 & 72 & 28.9 & 25.3 & 75 & Truce. \\
\hline 8 & 26.7 & 24.6 & 84 & 28.8 & 26 & 80 & 30.5 & 26.9 & 76 & 0 \\
\hline 9 & 26.9 & 25.1 & 86 & 29.4 & 26.4 & 79 & 28.6 & 25.4 & 77 & 0 \\
\hline 10 & 25 & 23.4 & 87 & 30.6 & 26.6 & 73 & 29.9 & 26.1 & 73 & Trace. \\
\hline 11 & 25.2 & 23.2 & 85 & 28.9 & 26.2 & 80 & 28 & 26 & 85 & 3 \\
\hline $12_{-}$ & 24.7 & 23.6 & 91 & 28.9 & 26.1 & 80 & 29.4 & 26.5 & 80 & Trace. \\
\hline 13 & 25.8 & 23.9 & 85 & 28.7 & 26.2 & 82 & 26 & 24.4 & 87 & 9 \\
\hline 14 & ------ & -- & - & --_-- & $\ldots$ & --- & - & - & --_- & Trace. \\
\hline $15_{-}$ & 26.1 & 23.8 & 82 & 29.3 & 26.4 & 79 & 30.7 & 26.2 & 69 & 0 \\
\hline 16 & 26.7 & 23.8 & 77 & $\ldots$ & $-\cdots$ & -- & -- & --- & -- & 0 \\
\hline $17-$ & $-\cdots$ & $-\cdots$ & --- & $-\cdots$ & --- & -- & & --- & & 0 \\
\hline 18 & --- & $-\cdots$ & --- & $-\cdots$ & - & ---- & $-\cdots$ & $-\cdots$ & $\cdots$ & 0 \\
\hline 19 & 27.2 & 24.3 & 78 & 29.4 & 26 & 75 & 28.6 & 25.5 & 77 & 0 \\
\hline 20 & 26.5 & 24.1 & 81 & 30 & 26.8 & 77 & 28.8 & 26 & 79 & 0 \\
\hline 21 & 27.2 & 25 & 83 & 29.8 & 25.9 & 72 & 30 & 26 & 71 & 0 \\
\hline 22 & 27.7 & 24.9 & 79 & 29.6 & 26 & 74 & 29.3 & 26.2 & 77 & 0 \\
\hline $23 \ldots$ & 26.3 & 24.2 & 83 & 30.6 & 26.2 & 70 & 32 & 26.7 & 64 & 0 \\
\hline 24 & 26.8 & 23.3 & 73 & 30.7 & 26.1 & 68 & 29.9 & 25.9 & 71 & 0 . \\
\hline 25 & 27.2 & 24.3 & 78 & 31.2 & 27.4 & 73 & 30 & 26.6 & 75 & 0 \\
\hline 26 & 26.6 & 23.9 & 79 & 30.1 & 26.3 & 73 & 30.4 & 26.3 & 71 & 0 \\
\hline $27 \ldots$ & 27.3 & 24.8 & 81 & 31.1 & 26.6 & 69 & 29 & 26.7 & 83 & 0 \\
\hline 28 & 26.9 & 24.1 & 78 & ---- & ---- & ------- & - & - & $-\ldots-$ & 0 \\
\hline 29 & 26.6 & 23.7 & 77 & 32.2 & 27.3 & 67 & 32.3 & 27.9 & 70 & 0 \\
\hline $30 \ldots$ & & & & & & --- & & & & \\
\hline Average. & $-\cdots$ & & 81.875 & ------ & $-\ldots$ & 74.9 & & & 75.32 & 12.5 \\
\hline
\end{tabular}




\section{ILLUSTRATIONS.}

\section{Plate I.}

Fia. 1. Transverse section of old root, showing continuously thickened endodermis and neighboring tissues.

2. Transverse section, 9 centimeters from tip of root, showing young endodermis, "e."

3. Transverse section of root 4.5 millimeters in diameter, 10 centimeters from tip, the hypodermal shell forming.

4. Transverse section, hypodermal shell of old root.

5. Longitudinal section, cortical parenchyma of young root in 5 per cent potassium nitrate, showing wrinkled walls.

(All figures magnified 160 diameters.)

Plate II.

Fig. 6. Root 8 millimeters in diameter, 1 centimeter from tip, surface view. ( 160 diameters.)

7. Same, longitudinal section of epidermis. (160 dianeters.)

8. Same, transverse section. (160 diameters.)

9. An old pneumathode. (1.25 diameters.)

10. Longitudinal section of young pneumathode: $S=$ stele, Co $=$ cortex, $\mathrm{Ca}=$ cap. $\quad(2.5$ diameters. $)$

- 11. Detail, area " $\mathrm{x}$ " in fig. 10. (20.5 diameters.)

12. Diagram of small pneumathode, showing relation to loose inner cortex of parent root. (20.5 diameters.)

13. Stellate cells, cortex of pneumathode. ( 87.5 diameters.)

14. Thickened cortical cells, base of old pneumathode. (87.5 diameters.)

\section{Plate III.}

Fig. 15. Transverse section of hinge, chlorophyll-bearing tissue indicated by stipelling. (87.5 diameters.)

16. Diagram of axis of leaf: $A=$ fibro-vascular bundle, $B=$ schlerenchyma sheath, $\mathrm{C}=$ upper epidermis and hypodermis, $\mathrm{D}=$ green parenchyma, $\mathrm{E}=$ hinge, $\mathrm{F}=$ nether epidermis and hypodermis. (20.5 diameters.)

17. Upper epidermis, transverse section. (160 dianeters.)

18. Same, longitudinal section. (160 diameters.)

19. Transverse section of stoma. (160 diameters.)

20. Tangential section, nethermost layer of green parenchyma; contents indicated only where bordering an intercellular space. (160 diameters.) 

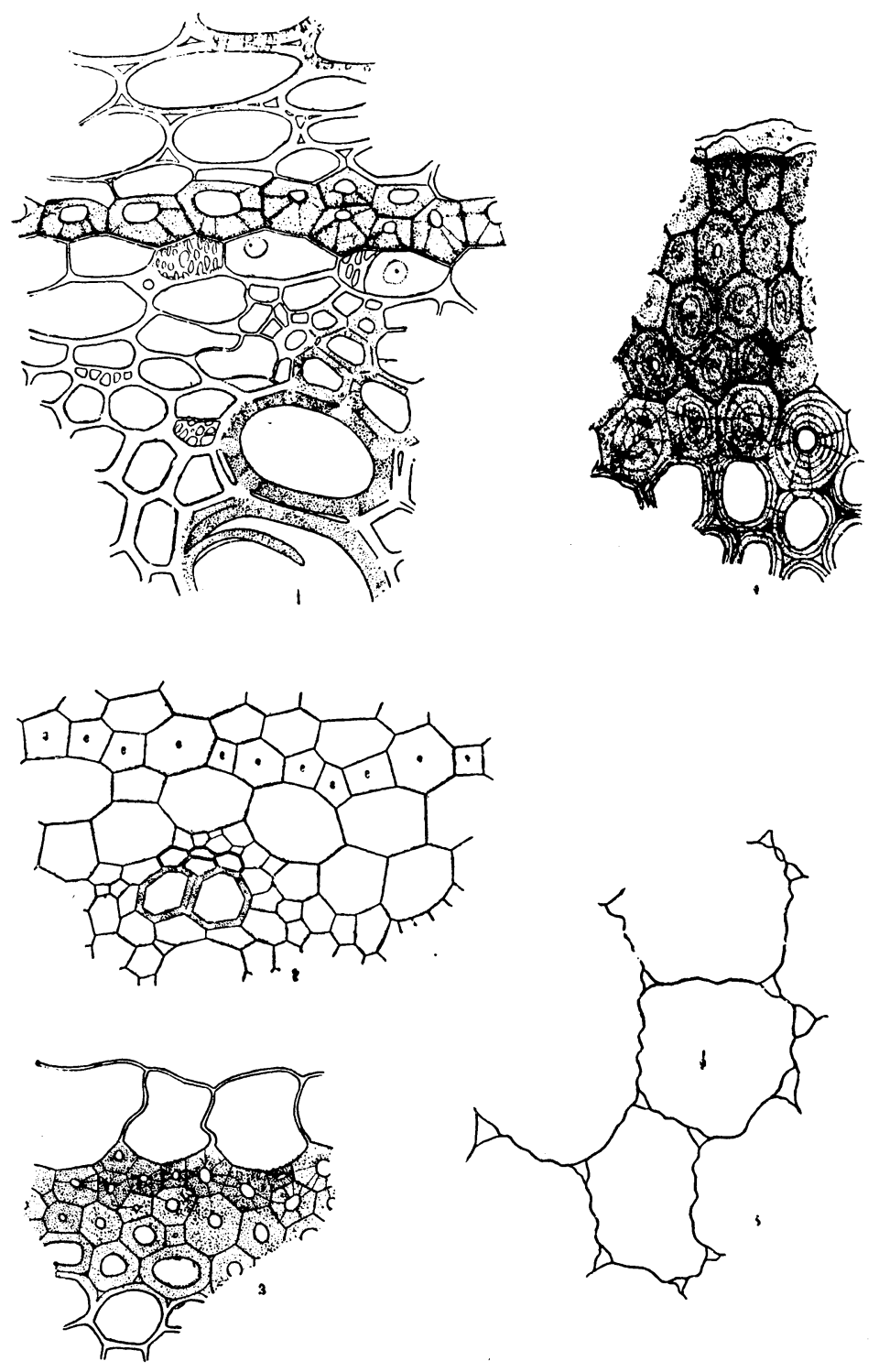

Plate I. 
COPEIAND: ON THE WATER REIationg, ETC.] [PHII. JoURN. Sici., VoI. I, No. 1.

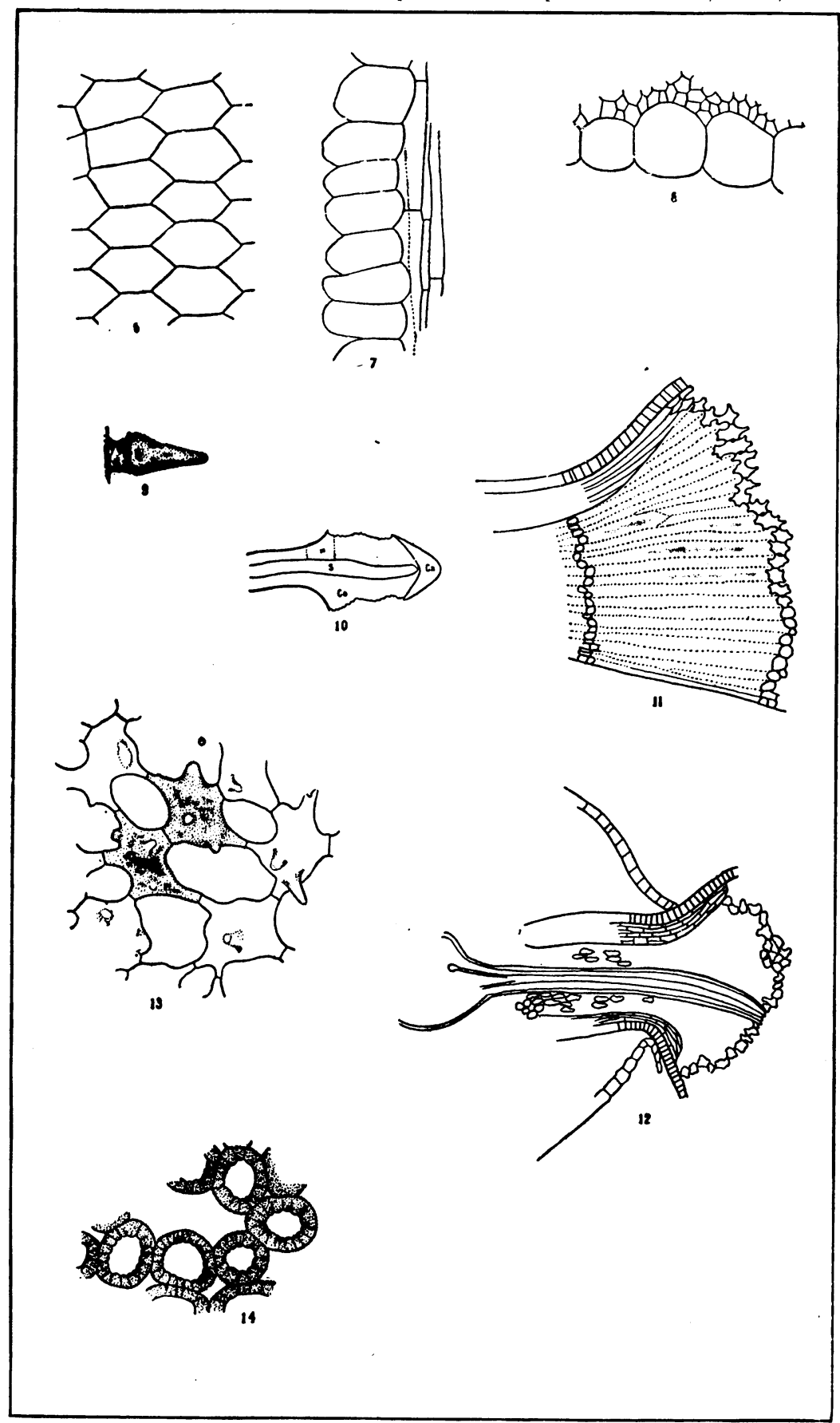

Plate II. 

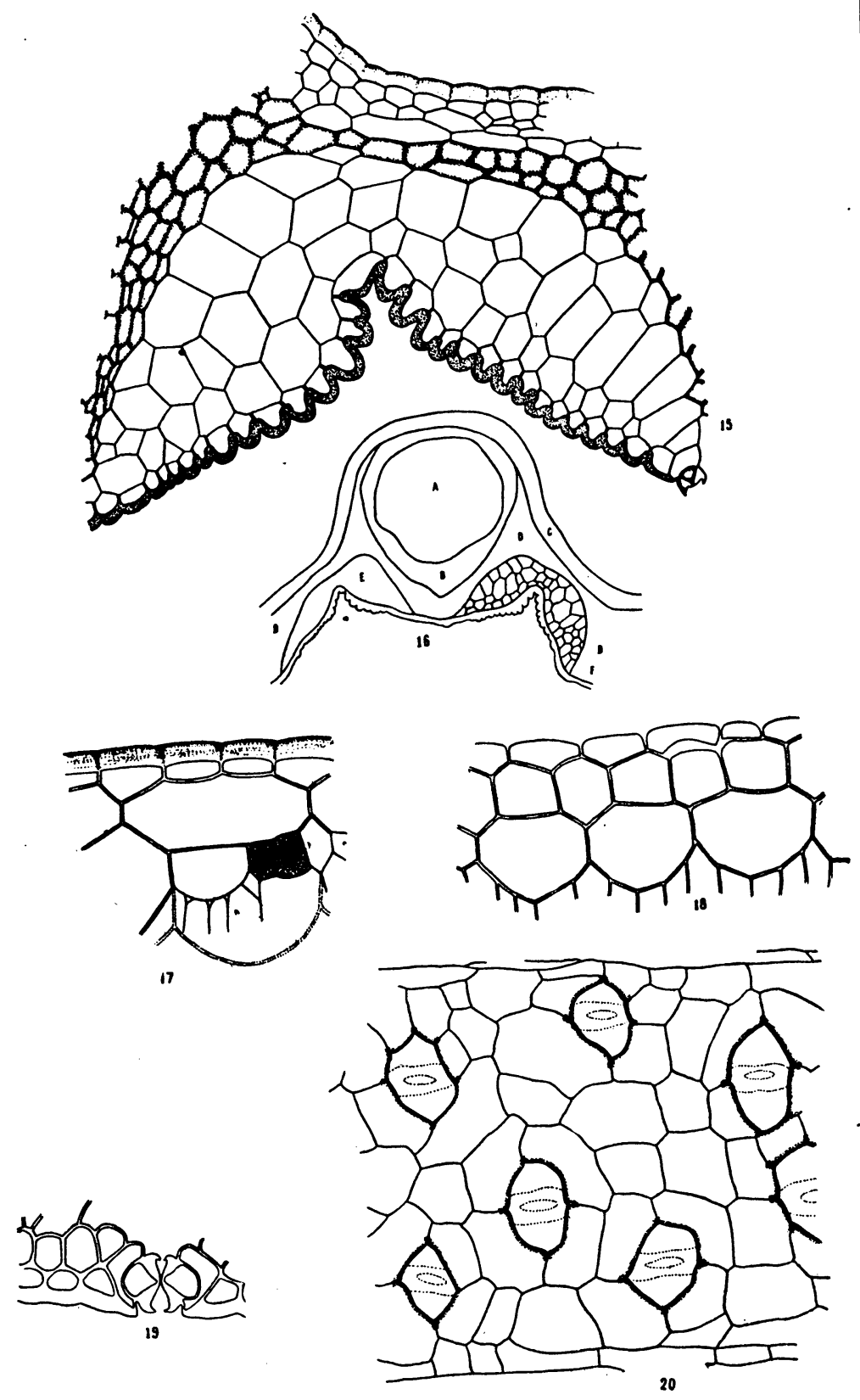

Plate III. 


\section{THE COCONUT AND ITS RELATION TO THE PRODUC- TION OF COCONUT OIL.}

By Herbert S. Wather.

(From the Chemical Laboratory, Bureau of Science.)

THE SOII.

Coconut production from the standpoint of the quality and quantity of the oil yielded has hitherto not been investigated, and it was decided to enter into this subject as fully as possible. The first problem which presented itself was the influence of the soil in which the trees grow on the yield of nuts, copra, and oil. It had been noticed for a long time that coconut trees growing near the seashore at San Ramon produced much more fruit than those standing farther inland, and it had also been stated that the former trees bear a better quality of nuts than the latter.

To determine how far these facts might be accounted for by a greater fertility of the soil near the sea, the following analyses were made of a number of soils in which coconut trees were growing, the samples being taken at the beach as well as farther inland, and two from Davao, where coconut trees flourish:

Analyses of San Ramon soils.

\begin{tabular}{|c|c|c|c|c|c|c|c|c|}
\hline Sample. & $\begin{array}{l}\text { Fine } \\
\text { earth. }\end{array}$ & Moisture. & $\begin{array}{c}\text { Loss on } \\
\text { ignition. }\end{array}$ & $\mathrm{P}_{2} \mathrm{O}_{5}$ & $\mathrm{~K}_{2} \mathrm{O}$. & N. & $\begin{array}{c}\text { Fine } \\
\text { earth, Cl. }\end{array}$ & $\begin{array}{c}\text { Coarse } \\
\text { earth, Cl. }\end{array}$ \\
\hline$A_{1}$ & $\begin{array}{r}60 \text { mesh. } \\
\quad 22.5\end{array}$ & 2.65 & 5.83 & 0.08 & 0.36 & 0.18 & 0.012 & 0.001 \\
\hline$A_{2}$ & 7 & 2.45 & 3.06 & .07 & .52 & .03 & .018 & .002 \\
\hline$A_{3}$ & 23 & 1.99 & 1.68 & .07 & .55 & .02 & .009 & .002 \\
\hline$B_{1}$ & 52 & 5.11 & 7.93 & .33 & .58 & .13 & .018 & .001 \\
\hline$B_{2}$ & 37.5 & 7.82 & 5.97 & .08 & .45 & .04 & .004 & .001 \\
\hline $\mathbf{B}_{3}$ & 45 & 7.33 & 6.03 & .08 & .48 & .03 & .006 & .001 \\
\hline $\mathrm{C}_{2}$ & 3.7 & 2.55 & 1.53 & .10 & .88 & .003 & & \\
\hline $\mathrm{C}_{3}$ & 2.1 & 2.65 & 1.45 & & & .02 & & \\
\hline$D_{1} \ldots$ & $\begin{array}{c}30 \text { mesh. } \\
38\end{array}$ & 2.52 & 1.35 & .11 & .18 & .01 & & \\
\hline$D_{2}$ & 26.9 & 2.96 & 1.71 & .11 & .62 & .004 & & \\
\hline $\mathrm{D}_{3} \ldots$ & 37.2 & 2.32 & 2. 29 & .07 & .65 & .01 & & \\
\hline $\mathrm{E}$ & $\begin{array}{r}40 \mathrm{mesh} . \\
43.6\end{array}$ & 7.6 & 1.79 & .08 & .40 & .05 & & \\
\hline F & 85.7 & 24 & 6.04 & .24 & .21 & .11 & & \\
\hline
\end{tabular}


Moisture on samples $F$ and $F$ determined at San Ramon; on all others at the laboratory, Manila.

Fine earth determined on original samples.

Loss on $\mathrm{N}, \mathrm{P}_{2} \mathrm{O}_{5}, \mathrm{~K}_{2} \mathrm{O}$ determined and percentages calculated to original sample on soil dried at $100^{\circ}-105^{\circ}$.

Soils marked " $A$ " were taken at a distance of 60 feet from the sea, $A_{1}$ being the surface, $A_{2} 18$ inches, and $A_{3} 3$ feet below.

The $B$ soils were taken from 2,800 feet inland, 40 feet above sea level, where trees were not bearing so well, and at the same depths as the A soils of corresponding numbers.

The $C$ soils were from the same place as those marked "A," but were taken at a greater depth so as to reach the locality of the deepest roots, $C_{2}$ being from

4 feet and $\mathrm{C}_{3}$ from 8 feet below the surface.

The $D$ soils were taken from the same place and depths as the $C$ soils.

Soil $E$ was taken at a depth of 3 to 4 feet, 6 feet distant from a very healthy 5-year-old tree near the sea.

Soil $F$ was taken at a depth of about 3 feet and about 1,800 feet from the sea, where trees do not bear so well.

Davao soils.

\begin{tabular}{|c|c|c|c|c|c|c|c|}
\hline Sample. & $\begin{array}{l}\text { Fine } \\
\text { earth. }\end{array}$ & $\begin{array}{l}\text { Mois- } \\
\text { ture. }\end{array}$ & $\begin{array}{c}\text { Loss on } \\
\text { ignition. }\end{array}$ & $\mathrm{P}_{2} \mathrm{O}_{5}$ & $\mathrm{~K}_{2} \mathrm{O}$. & N. & $\mathrm{CaO}$. \\
\hline I & $\begin{array}{c}40 \text { mesh. } \\
95\end{array}$ & 7.60 & 5.42 & 0.16 & 0.26 & 0.05 & 2.85 \\
\hline II & 91.9 & 1.30 & 1.42 & .11 & .13 & .03 & 2.06 \\
\hline
\end{tabular}

Soil marked "I" was taken at a distance of 50 yards from the Davao River about 1 mile inland from the sea, where trees were growing well.

Soil marked "II" was taken at the mouth of the Davao River about 50 feet from the sea. In this location a few young trees were doing fairly well.

Both samples were taken at a depth of about 1 foot.

Chemically, the results of these analyses show very little difference between the soils near the shore and those farther inland. The latter, contrary to what would be supposed, were found to be somewhat superior to the former, although neither could be called extremely fertile. Chlorine was determined in the first six of these samples, with the idea that this element might play some part in the better growth of trees near the sea, but the amounts found were so small as to be almost negligible. From these results it is evident that the inferior quality of the inland trees can not be explained by the analytical difference in the soils; neither does the salt from the sea appear to an appreciable extent, even around those trees which are actually growing on the beach.

However, the superior growth of trees near the sea might well be accounted for theoretically by the physical characteristics of the soil alone. For example, the soil marked " $\mathrm{E}$ " in the foregoing table is practically nothing but a very porous sand which, at a depth of 3 feet, is completely saturated with moisture; while $\mathrm{F}$ is a very stiff clay, such as the Spaniards formerly used for making bricks. While it is true that the latter contains more total moisture and plant nutriment than the 
former, the amount available to the tree is probably not by any means as great, owing to the difference in porosity. ${ }^{1}$ In view of the large amount of water necessary to the life of the coconut tree, one would naturally expect it to grow better in an easily permeable soil rather than in one from which water and soluble nutriment can only be taken up with difficulty. The objection has been raised that according to chemical analyses the soils near the sea do not appear to contain sufficient plant food to support life of any kind, much less that of a large and heavily productive tree like the coconut.

From analyses of coconuts made at this Bureau we have found that nuts from San Ramon contain nitrogen, potash, and phosphoric acid in approximately the following amounts :

\begin{tabular}{|c|c|c|c|}
\hline Part. & Nitrogen. & Potash. & $\begin{array}{l}\text { Phos- } \\
\text { phoric } \\
\text { acid. }\end{array}$ \\
\hline Husk _. & $\begin{array}{c}\text { Grams. } \\
1.609\end{array}$ & $\begin{array}{l}\text { Grams. } \\
\quad 3.915\end{array}$ & $\begin{array}{r}\text { Grams. } \\
0.017\end{array}$ \\
\hline Shell ... & 660 & .947 & .459 \\
\hline Mert -..-. & 4. 683 & 2.475 & 1.740 \\
\hline Milk__-_-_ & 1.542 & 1.313 & .171 \\
\hline Total & 8.494 & 8.650 & 2.387 \\
\hline
\end{tabular}

In 1 hectare of land on which about 173 trees are growing and producing, a total of about 7,000 nuts per annum may be expected. ${ }^{2}$ Under these conditions there is exhausted from the soil by the nuts alone a total of-

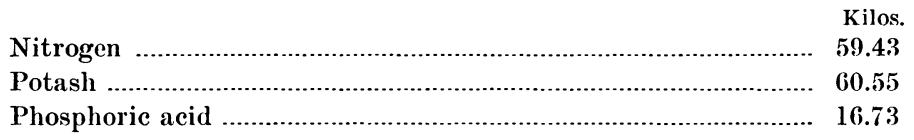

In addition to this there is a large weight of material withdrawn by falling leaves. Each tree on an average will lose annually 16 leaves, weighing about 3 kilos each, making about 8,300 kilos per year lost by 173 trees. Analysis shows that in this weight of dry leaves there is approximately-

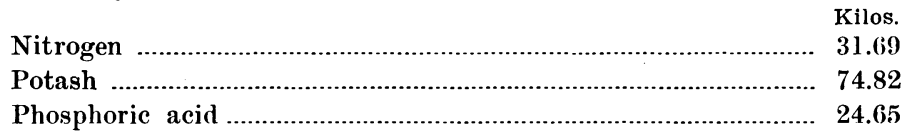

We have then a total annual drain on the soil per hectare of-

Nitrogen

Kilos.

Potash

91.12

Phosphoric acid

135.37

41.38

${ }^{1}$ See the paper by E. B. Copeland on the character of the roots of the Cocos.

${ }^{2}$ This is on the basis of 40 nuts per tree per annum, a very high average for San Ramon trees. 
Assuming these figures to be approximately correct, it would appear at first glance somewhat of a puzzle to determine low the tree manages to thrive and take up so much nourishment each year from a soil seemingly so devoid of fertility as that along the sea at San Ramon. However, when we consider the total amount of soil available to each tree, the problem becomes a simple one. The root mass of a coconut draws nutriment from a depth of at least 2 meters below the surface of the ground and outward on all sides for from $3 \frac{1}{2}$ to $6 \frac{1}{2}$ meters distance from its base. It thus comes in contact with an exceedingly large mass of material and it makes use of all the available nourishment therein.

In 1 hectare, or 10,000 square meters, of land there is available to the coconut trees planted thereon a total of at least 20,000 cubic meters of soil, or, if we allow a specific gravity of about two, 40,000,000 kilos.

From the table of analyses of San Ramon soils, we find that the soils near the sea average about as follows:

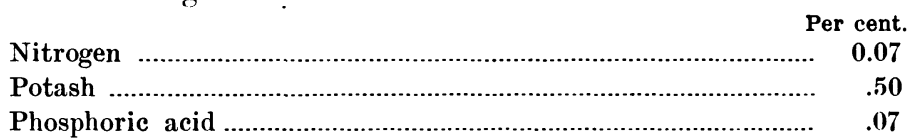

In 40,000,000 kilos we have-

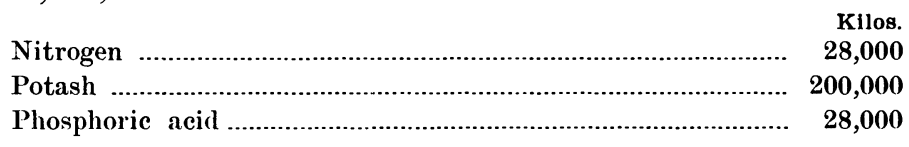

From the amount taken from the soil in each year, even though no fresh addition were made, we can calculate the number of years required completely to exhaust this soil of its plant food as follows:

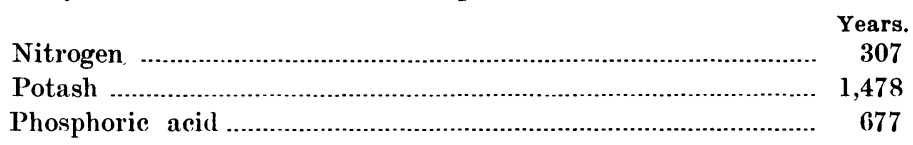

These figures are naturally only an approximation, but they show that even in a comparatively poor ground there exists more than an abundance of nourishment for the coconut tree, provided the soil itself is sufficiently porous and well watered. ${ }^{3}$

It seems very probable that in San Ramon at least, if not in most plantations along the seacoast, the nutritive material comes not from the soil in which the trees are actually growing but from an inexhaustible supply of water, laden with plant food, which is constantly seeping down from the higher ground toward the ocean. This underground water supply would account for the flourishing condition of trees in a sandy soil near the sea, even in times of drought, when individuals farther inland in higher, less permeable ground would be dying from want of water.

${ }^{3}$ See the paper by E. B. Copeland on the transpiration of the coconut and the amount of water taken up by an individual tree. 
Fertilization and irrigation.-In the case of the less permeable soils, artificial irrigation during the dry season would seem to be of the utmost importance, and any addition to the fertility of the land, either in the form of manure or of a chemical fertilizer, would probably be repaid by an increased yield of fruit. For soils near the sea, under conditions such as exist at San Ramon, irrigation is of course unnecessary excepting in times of extreme drought, and fertilization would be of doubtful advantage, as the trees in such a location seem to be growing under the best conditions possible without any attention whatsoever. Fertilizing material in such localities would probably be leached out and carried into the sea before it could be of much value to the trees.

THE NUT AND ITS OIL PRODUCTION.

The analytical methods used in compiling the accompanying tables were as follows: The weights, in grams, of husk, nut minus husk, shell, and milk were determined directly. To avoid loss by evaporation the meat itself was not weighed but was assumed to be the difference between the weight of the whole nut (minus the husk) and the combined weights of shell and milk.

Copra.-The meat from each nut was allowed to dry in the air over night, so as to assume a fairly constant weight, and was then weighed directly; 25 grams were then cut into fine pieces and dried to constant weight at $100^{\circ} \mathrm{C}$. for the determination of anhydrous copra, the latter being calculated back to per cent in the fresh meat. To approximate the amount of commercial copra obtainable, an addition of about 10 per cent should be made because of the water ordinarily contained in this product.

Oil.-The anhydrous copra prepared at San Ramon was sealed in glass bottles and shipped to Manila for analysis, the majority of the oil determinations being made by Mr. George F. Richmond, of this laboratory. Before this time much work had been done in devising a method for the rapid and accurate estimation of oil in copra. It was found to be almost impossible to make a complete extraction by the ordinary method of cutting fine pieces and extracting with ether in a Soxhlet cone. Even after the apparatus had been running for forty hours, a small increase in weight was obtained by extracting for eight hours more. Grinding with sand and then extracting with ether produced some improvement. Extraction with hot chloroform alone took out a little more oil, but it was necessary to continue the operation for at least sixteen hours. The method finally used was as follows:

A 2-gram sample was intimately ground with fine sand in a glass mortar, the mixture transferred to a Soxhlet cone, the mortar washed two or three times with fresh sand, and then finally wiped with fat-free cotton. The extraction with hot chloroform takes three hours.

The chloroform is then distilled and the remaining oil dried to constant weight at $100^{\circ} \mathrm{C}$. Experiment demonstrated that practically all the oil was extracted in two hours. The chloroform extract made in this way proved to be entirely soluble in absolute ether. The sand used was prepared from ordinary sea sand by taking all which went through a 30 -mesh and which was retained on a 100 mesh sieve, heating this product for some time to destroy organic matter and then afterwards extracting with chloroform. 
Age in reference to quality of the nut.-One of the first and most important of the problems which presented itself was to determine the effect of the age and the relative maturity of the nut on the percentage of its various constituents; in other words, to find out the most favorable time for opening a nut to obtain the largest and best yield of copra and of oil. With this end in view, the following analyses were made of four serics of ten nuts each:

Series I consisted of 10 nuts selected from a pile which had just been picked from the trees and which were ready to be used for making copra. These nuts were all fairly ripe, although the husks were still green in color and full of water.

Series II was made up of 10 nuts from the same pile as Series I, but all were very ripe. The husks were dry and of a dead-brown color.

Series III represents nuts which had been selected for seed and set out to sprout about three months before the time of analysis. They each contained a small embryo or "foot" and had green sprouts protruding to a height of about 6 inches. The husks had absorbed a large amount of water while lying on the ground.

Series IV was a rather abnormal lot of nuts which had been set out for sced similarly to those in Series III, but for some reason the individual ones had failed to sprout, although they had been standing for six months. The meat had become somewhat softened and in several cases it was discolored and possessed a bad odor. To a greater extent than any other this series shows what large variations may exist among nuts of the same age. 
Seriws I.-Ten muts, fresh from trees but fairly ripe (all green huskix).

\begin{tabular}{|c|c|c|c|c|c|c|c|c|c|c|c|c|c|}
\hline \multirow[b]{2}{*}{ No. } & \multirow{2}{*}{\multicolumn{2}{|c|}{ 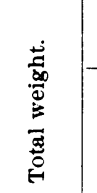 }} & \multicolumn{2}{|c|}{ Husk. } & \multicolumn{2}{|c|}{$\begin{array}{l}\text { Nut minus } \\
\text { husk. }\end{array}$} & \multicolumn{3}{|c|}{ Shell. } & \multicolumn{4}{|c|}{ Meat. } \\
\hline & & & 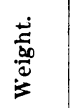 & 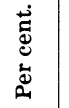 & 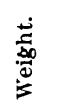 & 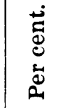 & $\frac{2}{9}$ & & 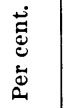 & 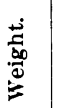 & 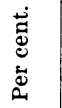 & 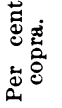 & 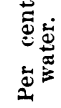 \\
\hline 1 & \multicolumn{2}{|c|}{2,702} & 1,160 & 42.9 & 1,542 & 57.1 & \multicolumn{2}{|c|}{290} & 10.8 & 725 & 26.8 & 45. 2 & 54.8 \\
\hline $2 \ldots$ & \multicolumn{2}{|c|}{2,725} & 1,265 & 46.4 & 1,460 & 53.6 & \multicolumn{2}{|c|}{330} & 12.1 & 590 & 21.7 & 43.9 & 56.1 \\
\hline 3 & \multicolumn{2}{|c|}{3,039} & 1,510 & 49.7 & 1,529 & 50.3 & \multicolumn{2}{|c|}{395} & 13.0 & 639 & 21.0 & 54.8 & 45.2 \\
\hline 4. & \multicolumn{2}{|c|}{3,182} & 1,547 & 48.6 & 1,635 & 51.4 & \multicolumn{2}{|c|}{340} & 10.7 & 585 & 18.4 & 32.8 & 67.2 \\
\hline 5 & \multicolumn{2}{|c|}{2,830} & 1,300 & 45.9 & 1,530 & 54.1 & \multicolumn{2}{|c|}{299} & 10.6 & 606 & 21.4 & 44.5 & 5.5 .5 \\
\hline 6 & \multicolumn{2}{|c|}{2,316} & 1,106 & 47.8 & 1,210 & 52.2 & \multicolumn{2}{|c|}{239} & 10.3 & 541 & 23.4 & 46.1 & 53.9 \\
\hline $7 \ldots$ & 2, & & 1,157 & 40.3 & 1,715 & 59.7 & 3 & 38 & 11.8 & 652 & 22.7 & 43.9 & 56.1 \\
\hline $8--$ & 2,9 & & 1,350 & 46.1 & 1,580 & 53.9 & 3 & 45 & 11.8 & 580 & 19.8 & 48.5 & 51.5 \\
\hline 9 & 2,1 & & 925 & 44.0 & 1,175 & 56.0 & 2 & 60 & 12.4 & 505 & 24.1 & 53.2 & 46.8 \\
\hline 10 & 3,7 & & 1,915 & 50.6 & 1,872 & 49.4 & 3 & $9 \tilde{5}$ & 10.4 & 717 & 18.9 & 51.7 & 48.3 \\
\hline Average ---- & 2,8 & & 1,323 & 46.2 & 1,525 & 53.8 & $3:$ & 23 & 11.4 & 614 & 21.8 & 46.5 & 53.5 \\
\hline & & ra $(a$ & anhydro & ous). & $\mathrm{Mi}$ & & & il. & & $\begin{array}{l}\text { leula } \\
\text { nut }\end{array}$ & $\begin{array}{l}\text { ed to } \\
\text { ee fro }\end{array}$ & $\begin{array}{l}\text { ree } \\
\text { hus }\end{array}$ & \\
\hline No. & $\begin{array}{l}\stackrel{+}{0} \\
\stackrel{00}{0} \\
\dot{0}\end{array}$ & 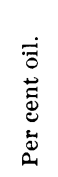 & $\begin{array}{l}\dot{a} \\
\vec{\Xi} \\
\ddot{0} \\
\ddot{d} \\
\dot{0} \\
\dot{0}\end{array}$ & 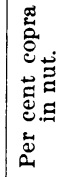 & 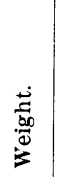 & 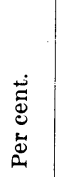 & 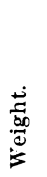 & $\begin{array}{l}\dot{0} \\
\dot{0} \\
\dot{0} \\
0 \\
0\end{array}$ & 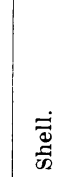 & $\underset{⿱ 乛}{\stackrel{\Xi}{*}}$ & 芯 & 尝 & $\dot{\overrightarrow{0}}$ \\
\hline $1 \ldots$ & 327 & 67.2 & 32.8 & 12.1 & 527 & 19.5 & 220 & 8.1 & 18.8 & 47.0 & 21.2 & 34.2 & 14.3 \\
\hline 2 & 259 & 66.4 & 33.6 & 9.5 & 540 & 19.8 & 172 & 6.3 & 22.6 & 40.4 & 17.7 & 37.0 & 11.8 \\
\hline 3 - & 351 & 69.3 & 30.7 & 11.5 & 495 & 16.3 & 243 & 8.0 & 25.8 & 41.8 & 23.0 & 32.4 & 15.9 \\
\hline $4 \ldots$ & 192 & 59.8 & 40.2 & 6.0 & 710 & 22.3 & 115 & 3.6 & 20.8 & 35.8 & 11.7 & 43.4 & 7.0 \\
\hline 5 & 270 & 63.4 & 36.6 & 9.5 & 625 & 22.1 & 171 & 6.0 & 19.5 & 39.6 & 17. 7 & 40.9 & 11.2 \\
\hline 6 & 250 & 64.9 & 35.1 & 10.8 & 430 & 18.6 & 162 & 7.0 & 19.8 & 44.7 & 20.7 & 35.5 & 13.4 \\
\hline $7 \ldots$ & 287 & 62.3 & 37.7 & 10.0 & 725 & 25.2 & 179 & 6.2 & 19. 7 & 38.0 & 16.7 & 42.3 & 10.4 \\
\hline 8 & 281 & 63.0 & 37.0 & 9.6 & 655 & 22.3 & 177 & 6.0 & 21.8 & 36.7 & 17.8 & 41.5 & 11.2 \\
\hline 9 & 269 & 65.3 & 34.7 & 12.8 & 410 & 19.5 & 176 & 8.4 & 22.1 & 43.0 & 22.9 & 34.9 & 15.0 \\
\hline 10 & 371 & 68.7 & 31.3 & 9.8 & 760 & 20.1 & 255 & 6.7 & 21.1 & 38.3 & 19.8 & 40.6 & 13.6 \\
\hline Average ---- & 286 & 65.0 & 35.0 & 10.2 & 588 & 20.6 & 187 & 6.6 & 21.2 & 40.5 & 18.9 & 38.3 & 12.4 \\
\hline
\end{tabular}


SERIEs II.-Ten muts, very ripe (deud-brou'n huakx).

[Selected from pile of several thousand.]

\begin{tabular}{|c|c|c|c|c|c|c|c|c|c|c|c|c|c|}
\hline \multirow[b]{2}{*}{ No. } & \multirow{2}{*}{\multicolumn{2}{|c|}{ 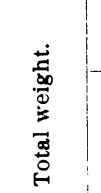 }} & \multicolumn{2}{|c|}{ Husk. } & \multicolumn{2}{|c|}{$\begin{array}{c}\text { Nut minus } \\
\text { husk. }\end{array}$} & \multicolumn{3}{|c|}{ Shell. } & \multicolumn{4}{|c|}{ Meat. } \\
\hline & & & $\frac{\stackrel{5}{*}}{\frac{\infty}{20}}$ & 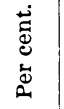 & 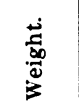 & 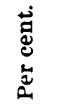 & 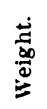 & & : & 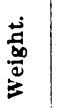 & 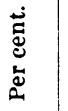 & 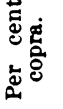 & 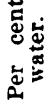 \\
\hline 1 -- & \multicolumn{2}{|c|}{2,616} & 665 & 25.4 & 1,951 & 74.6 & 352 & \multicolumn{2}{|c|}{13.5} & 774 & 29.6 & 43.5 & 56.5 \\
\hline 2 & \multicolumn{2}{|c|}{1,935} & 350 & 18.1 & 1,585 & 81.9 & 328 & \multicolumn{2}{|c|}{17.0} & 602 & 31.1 & 59.9 & 40.1 \\
\hline 3 & \multicolumn{2}{|c|}{2,025} & 460 & 22.7 & 1,565 & 77.3 & 262 & \multicolumn{2}{|c|}{13.0} & 644 & 32.5 & 50.9 & 49.1 \\
\hline 4 & \multicolumn{2}{|c|}{1,681} & 332 & 19.7 & 1,349 & 80.3 & 283 & \multicolumn{2}{|c|}{16.8} & 596 & 35.5 & 51.5 & 48.5 \\
\hline 5 & \multicolumn{2}{|c|}{2,070} & 480 & 23.2 & 1,590 & 76.8 & 320 & \multicolumn{2}{|c|}{15.5} & 585 & 28.2 & 51.6 & 48.4 \\
\hline 6 & \multicolumn{2}{|c|}{2,192} & 647 & 29.5 & 1,545 & 70.5 & 380 & \multicolumn{2}{|c|}{17.4} & 715 & 32.6 & 56.3 & 43.7 \\
\hline 7 & \multicolumn{2}{|c|}{2,945} & 460 & 15.6 & 2,485 & 84.4 & 430 & \multicolumn{2}{|c|}{14.6} & 980 & 33.3 & 42.4 & 57.6 \\
\hline $8 \ldots$ & \multicolumn{2}{|c|}{1,948} & 437 & 22.4 & 1,511 & 77.6 & 330 & \multicolumn{2}{|c|}{17.0} & 641 & 32.9 & 56.9 & 43.1 \\
\hline $9 \ldots$ & \multicolumn{2}{|c|}{2,049} & 500 & 24.4 & 1,549 & 75.6 & 309 & \multicolumn{2}{|c|}{ 15. 1} & 675 & 32.9 & 51.7 & 48.3 \\
\hline 10 & \multicolumn{2}{|c|}{1,735} & 425 & 24.5 & 1,310 & 75.5 & 260 & \multicolumn{2}{|c|}{15.0} & 590 & 34.0 & 54.4 & 45.6 \\
\hline Average..-- & \multicolumn{2}{|c|}{2,120} & 476 & 22.6 & 1,644 & 77.5 & 326 & \multicolumn{2}{|c|}{15.5} & 680 & 32.3 & 52.0 & 48.0 \\
\hline \multirow[b]{2}{*}{ No. } & Co & $\mathrm{ra}(\mathrm{a}$ & hydre & us). & Mil & & Oi & il. & & $\begin{array}{l}\text { aleu } \\
\text { nut }\end{array}$ & d to & $\begin{array}{l}\text { r ce } \\
\text { hu }\end{array}$ & in \\
\hline & $\begin{array}{l}\frac{+1}{000} \\
\frac{0}{0} \\
5\end{array}$ & 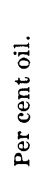 & 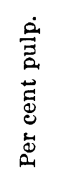 & 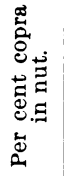 & 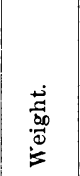 & 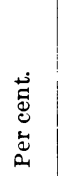 & 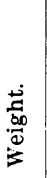 & 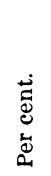 & 离 & 菾 & 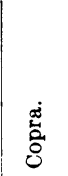 & $\dot{\vec{z}}$ & $\dot{\vec{\sigma}}$ \\
\hline 1 & 337 & 54.0 & 46.0 & 12.9 & 825 & 31.5 & 182 & 7.0 & 18.0 & 39.7 & 17.3 & 42.3 & 9.3 \\
\hline $2-$ & 360 & 66.4 & 33.6 & 18.6 & 655 & 33.8 & 239 & 12.3 & 20.7 & 38.0 & 22.7 & 41.3 & 15.1 \\
\hline $3--$ & 328 & 61.7 & 38.3 & 16.2 & 659 & 31.8 & 202 & 10.0 & 16.7 & 41. 2 & 21.0 & 42.1 & 12.9 \\
\hline 4 & 307 & 62.2 & 37.8 & 18.3 & 470 & 28.0 & 191 & 11.4 & 21.0 & 44.2 & 22.8 & 34.8 & 14.2 \\
\hline 5 & 302 & 59.6 & 40.4 & 14.6 & 685 & 33.1 & 180 & 8.7 & 20.1 & 36.8 & 19.0 & 43.1 & 11.3 \\
\hline 6 & 402 & 58.9 & 41.1 & 18.3 & 450 & 20.5 & 237 & 10.8 & 24.6 & 46.3 & 26.0 & 29.1 & 15.3 \\
\hline $7 \ldots$ & 415 & 58.1 & 41.9 & 14.1 & $\$ 1,075$ & 36.5 & 241 & 8.2 & 17.3 & 39.4 & 16.7 & 43.3 & 9.7 \\
\hline $8 \ldots$ & 365 & 66.3 & 33.7 & 18.7 & 540 & 27.7 & 242 & 12.4 & 21.8 & 42.4 & 24.2 & 35.8 & 16.0 \\
\hline 9 & 349 & 63.3 & 36.7 & 17.0 & 565 & 27.6 & 221 & 10.8 & 19.9 & 43.6 & 22.5 & 36.5 & 14.3 \\
\hline 10 & 321 & 63.1 & 36.9 & 18.5 & 460 & 26.5 & 203 & 11.7 & 19.9 & 45.0 & 24.5 & 35.1 & 15.5 \\
\hline Average_--- & 349 & 61.4 & 38.6 & 16.7 & 638 & 29.7 & 214 & 10.3 & 20.0 & 41.7 & 21.7 & 38.3 & 13.4 \\
\hline
\end{tabular}

a Milk very turbid. 
SERIES III.-Nuts stored three months, just beginning to sprout.

\begin{tabular}{|c|c|c|c|c|c|c|c|c|c|c|c|c|c|}
\hline \multirow[b]{2}{*}{ No. } & \multirow{2}{*}{ 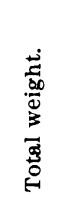 } & \multirow[b]{2}{*}{$\begin{array}{l}\dot{0} \\
\text { 至 } \\
\text { 章 }\end{array}$} & \multicolumn{3}{|c|}{ Husk. } & \multicolumn{2}{|c|}{$\begin{array}{l}\text { Nut minus } \\
\text { husk. }\end{array}$} & \multicolumn{2}{|c|}{ Shell. } & \multicolumn{4}{|c|}{ Meat. } \\
\hline & & & $\frac{\dot{3}}{30}$ & \multicolumn{2}{|c|}{ 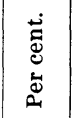 } & 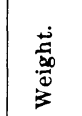 & 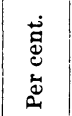 & $\frac{\frac{1}{30}}{\frac{30}{3}}$ & 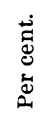 & $\frac{\dot{\vec{n}}}{\dot{a}}$ & 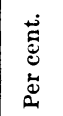 & 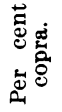 & 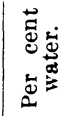 \\
\hline 1 & 2,935 & 15 & 1,31 & 44 & & 622 & 55.3 & 326 & 11.1 & 711 & 24.2 & 57.2 & 42.8 \\
\hline 2 & 2,133 & 25 & 1,06 & 49 & & 073 & 50.3 & 232 & 10.9 & 553 & 25.9 & 50.7 & 49.3 \\
\hline 3 & 1,966 & 15 & 72 & 37 & & 237 & 62.9 & 275 & 34.0 & 557 & 28.3 & 55.7 & 44.3 \\
\hline $4 \ldots$ & 1,666 & 10 & 56 & 33 & & 101 & 66.1 & 242 & 14.5 & 509 & 30.6 & 52.8 & 47.2 \\
\hline 5 & 2,791 & 20 & 1,65 & 59 & & 138 & 40.8 & 232 & 8.3 & 561 & 20.1 & 49.9 & 50.1 \\
\hline 6 & 2,537 & 5 & 1,39 & 55 & & 142 & 45.0 & 285 & 11.2 & 505 & 19.9 & 52.8 & 47.2 \\
\hline 7 & 2,664 & 50 & 97 & 36 & & 691 & 63.5 & 299 & 11.2 & 752 & 28.2 & 49.9 & 50.1 \\
\hline $8 \ldots$ & 3,993 & 5 & 2,73 & 68 & & 262 & 31.6 & 281 & 7.0 & 536 & 13.4 & 51.5 & 48.5 \\
\hline 9 & 5,062 & 15 & 3,11 & 61 & & 947 & 38.5 & 398 & 7.9 & 849 & 16.8 & 40.3 & 59.7 \\
\hline 10 & 2,300 & 10 & 1,16 & 50 & 4 & 140 & 49.6 & 273 & 11.9 & 540 & 23.5 & 51.7 & 48.3 \\
\hline Average .-.- & 2,805 & 17 & 1,46 & 49 & & 335 & 50.4 & 284 & 10.8 & 607 & 23.1 & 51.3 & 48.7 \\
\hline \multirow[b]{2}{*}{ No. } & \multicolumn{4}{|c|}{ Copra (anhydrous). } & \multicolumn{2}{|c|}{ Milk. } & \multicolumn{2}{|c|}{ Oil. } & \multicolumn{5}{|c|}{$\begin{array}{l}\text { Calculated to per cent in } \\
\text { nut free from husk. }\end{array}$} \\
\hline & 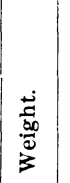 & 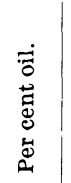 & 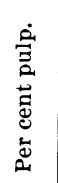 & 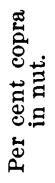 & $\frac{\stackrel{5}{5}}{30}$ & 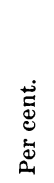 & 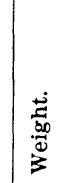 & 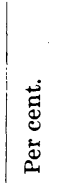 & 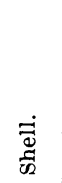 & 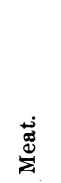 & 通 & 总 & $\dot{\sigma}$ \\
\hline 1 & 407 & 62.4 & 37.6 & 13.9 & 570 & 19.4 & 254 & 8.7 & 20.1 & 43.8 & 25.1 & 35.2 & 15.7 \\
\hline $2 \ldots$ & 280 & 59.5 & 40.5 & 13.1 & 263 & 12.3 & 167 & 7.8 & 21.6 & 51.5 & 26.1 & 24.5 & 15.5 \\
\hline $3 \ldots$ & 311 & 63.8 & 36.2 & 15.8 & 390 & 19.8 & 199 & 10.1 & 22.2 & 44.9 & 25.2 & 31.5 & 16.0 \\
\hline $4 \ldots$ & 269 & 63.6 & 36.4 & 16.1 & 340 & 20.4 & 171 & 10.3 & 22.0 & 46.2 & 24.4 & 30.9 & 15.5 \\
\hline 5 - & 280 & 65.1 & 34.9 & 10.0 & 325 & 11.6 & 182 & 6.5 & 20.4 & 49.3 & 24.6 & 28.6 & 16.0 \\
\hline 6 & 266 & 58.7 & 41.3 & 10.5 & 347 & 13. 7 & 156 & 6.2 & 25.0 & 44.2 & 23.3 & 30.4 & 13.7 \\
\hline 7 & 375 & 60.0 & 40.0 & 14.1 & 590 & 22.2 & 225 & 8.4 & 17.7 & 44.5 & 22.2 & 34.9 & 13.3 \\
\hline 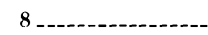 & 276 & 60.6 & 39.4 & 6.9 & 440 & 11.0 & 167 & 4.2 & 22.2 & 42.5 & 21.9 & 34.9 & 13.3 \\
\hline 9 & 342 & 62.2 & 37.8 & 6.8 & 685 & 13.5 & 212 & 4.2 & 20.4 & 43. 6 & 17.6 & 35.2 & 10.9 \\
\hline 10 & 279 & 62.1 & 37.9 & 12.1 & 317 & 13.8 & 173 & 7.5 & 24.0 & 47.4 & 24.5 & 27.8 & 15.2 \\
\hline Average & 309 & 61.8 & 38.2 & 11.9 & 427 & 15.8 & 191 & 7.4 & 21.6 & 45.8 & 23.5 & 31.4 & 14.5 \\
\hline
\end{tabular}


SERIEs IV. - Nints stored six months which had mot sprouted.

[Most nuts of this age have sprouts 20 to 30 centimeters long.]

\begin{tabular}{|c|c|c|c|c|c|c|c|c|c|c|c|c|c|}
\hline \multirow[b]{2}{*}{ No. } & \multirow{2}{*}{ 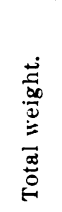 } & \multirow[b]{2}{*}{ 㻤 } & \multicolumn{3}{|c|}{ Husk. } & \multicolumn{2}{|c|}{$\begin{array}{l}\text { Nut minus } \\
\text { husk. }\end{array}$} & \multicolumn{2}{|c|}{ Shell. } & \multicolumn{4}{|c|}{ Mert. } \\
\hline & & & & 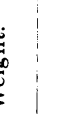 & بـ & 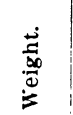 & 苞 & $\frac{\stackrel{3}{0}}{30}$ & 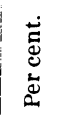 & 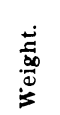 & 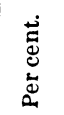 & 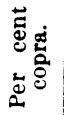 & 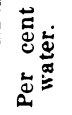 \\
\hline $1 \ldots$ & 2,908 & \multicolumn{2}{|c|}{$\underset{6}{\text { Grams. }}$} & 1,272 & 43. 7 & 1,636 & 56.3 & 367 & 12.6 & 683 & 23.5 & 51.3 & 48.7 \\
\hline $2 \ldots$ & 2,541 & \multirow{2}{*}{$-\cdots---$} & \multicolumn{2}{|c|}{1,382} & 54.4 & 1,159 & 45. 6 & 197 & 7.8 & 492 & 19.3 & 33.0 & 67.0 \\
\hline $3_{-\ldots}$ & 3,419 & & \multicolumn{2}{|c|}{1,743} & 51.0 & 1,676 & 49.0 & 360 & 10.5 & 666 & 19.5 & 44.1 & 55. 9 \\
\hline $4 \ldots$ & 3,154 & \multicolumn{2}{|c|}{$\mid \begin{array}{l}-\cdots+-- \\
-\cdots-n\end{array}$} & 1,462 & 46.3 & 1,692 & 53.7 & 277 & 8.8 & 800 & 25.4 & 51.3 & 48.7 \\
\hline 5 & 2,023 & & \multicolumn{2}{|c|}{1,123} & 55.5 & 900 & 44.5 & 252 & 12.5 & 500 & 24.7 & 54.7 & 45.3 \\
\hline $6_{-}$ & 2,276 & & \multicolumn{2}{|c|}{1,228} & 54.0 & 1,048 & 46.0 & 160 & 7.0 & 478 & 21.0 & 33.2 & 66.8 \\
\hline $7_{-}$ & 3,116 & & \multicolumn{2}{|c|}{1,939} & 62.2 & 1,176 & 37.8 & 260 & 8.4 & 536 & 17.2 & 37.3 & 62.7 \\
\hline 8 & 2,403 & & \multicolumn{2}{|c|}{1,443} & 60.1 & 960 & 39.9 & 180 & 7.5 & 453 & 18.8 & 29.3 & 70.7 \\
\hline 9 & 3,238 & & \multicolumn{2}{|c|}{$-1,800$} & 55.6 & 1,438 & 44.4 & 262 & 8.1 & 696 & 21.5 & 34.9 & 65.1 \\
\hline 10 & 3,585 & & \multicolumn{2}{|c|}{2,225} & 2.1 & 1,360 & 37.9 & 272 & 7.6 & 638 & 17.8 & 38.2 & 61.8 \\
\hline Average & 2,866 & - & 1, & 62 & 4.5 & 1,305 & 45.5 & 259 & 9.1 & 594 & 20.9 & 40.7 & 59.3 \\
\hline & Copr & $\mathrm{ra}(\mathrm{an}$ & hydro & us). & & filk. & $\mathrm{Oi}$ & & & nut $f$ & $\begin{array}{l}\text { to } 1 \\
\text { fror }\end{array}$ & $\begin{array}{l}r \text { cent } \\
\text { husk. }\end{array}$ & in \\
\hline No. & 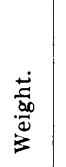 & 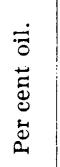 & 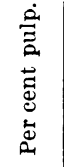 & 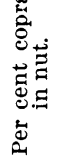 & 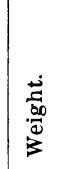 & 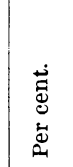 & 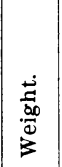 & 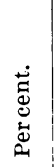 & 㐫 & 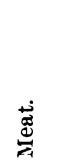 & 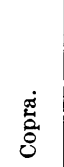 & $\underset{3}{3}$ & $\ddot{0}$ \\
\hline $1 \ldots$ & 350 & 61.4 & 38.6 & 12.1 & 580 & 20.1 & 216 & 7.4 & 22.5 & 41.6 & 21.4 & 35.5 & 13.1 \\
\hline $2 \ldots$ & 170 & 67.7 & 32.3 & 6.7 & 470 & 18.5 & 115 & 4.5 & 17.0 & 42.5 & 14.7 & 40.5 & 9.9 \\
\hline $3 \ldots$ & 294 & 69.0 & 31.0 & 8.6 & 650 & 19.0 & 203 & 5.9 & 21.5 & 39.7 & 17.5 & 38.8 & 12.1 \\
\hline 4 & 410 & 68.4 & 31.6 & 13.0 & 615 & 19.5 & 281 & 9.9 & 16.4 & 47.3 & 24.2 & 36.3 & 16.6 \\
\hline $\bar{j}$ & 274 & 74.4 & 25.6 & 13.5 & 143 & 7.1 & 204 & 10.1 & 28.0 & 55.6 & 38.3 & 15.9 & 22.7 \\
\hline 6 & 136 & 56.3 & 43.7 & 6.0 & 410 & 18.0 & 77 & 3.4 & 15.3 & 45.6 & 13.0 & 39.1 & 7.3 \\
\hline 7 - & 200 & 66.2 & 33.8 & 6.4 & 380 & 12.2 & 132 & 4.2 & 22.1 & 45.6 & 17.0 & 32.3 & 11.3 \\
\hline $8 \ldots$ & 132 & 74.1 & 25.9 & 5.5 & 327 & 13.6 & 98 & 4.1 & 18.7 & 47.2 & 13.8 & 34.1 & 10.2 \\
\hline 9 & 242 & 63.4 & 36.6 & 7.5 & 480 & 14.8 & 153 & 4.7 & 18.2 & 48.4 & 16.8 & 33.4 & 10.7 \\
\hline 10 & 244 & 58.5 & 41.5 & 6.8 & 450 & 12.5 & 143 & 4. 0 & 20.0 & 46.9 & 17.9 & 33.1 & 10.5 \\
\hline Average.-.-- & 245 & 65.9 & 34.1 & 8.6 & 451 & 15.5 & 162 & 5.8 & 20.0 & 46.0 & 19.5 & 34.0 & 12.4 \\
\hline
\end{tabular}

The variation among individual nuts in the foregoing analyses was rather greater than had been expected, and it is doubtful if even an average of ten nuts gives more than an approximation of their true value at a given age.

$36540-5$ 
However, considering the average percentages as calculated to the nut free from husk, there appears a gradual increase in the proportion of meat, copra, and oil from Series I to Series III, with a corresponding decrease in the percentage of milk, indicating that the meat is becoming firmer, is losing some water and gaining oil, as the nut increases in age. In Series IV, those nuts which had been kept for six months, the meat remains practically the same in amount, but there is a marked drop in the proportion of copra and oil, probably due to decomposition or other changes which are beginning to take place in the meat. However, No. 5 of this series, a nut in which decomposition had already set in, shows an abnormally high percentage of both copra and oil, a fact which is very hard to account for, although it is possible that this individual may have been still higher in these substances before decomposition began. In both Series I and IV the percentage of oil in the anhydrous copra is considerably higher than it is in II and III, though this is more than counterbalanced by a much lower proportion of copra in the meat. Both in very fresh and in overripe nuts there is a considerable deficiency in oil, but the principal loss is in the amount of copra to be obtained, this result being due to a higher percentage of water as compared with solid matter in the meat. In all these nuts it will be noticed that the proportion of shell to the whole nut varies but little.

Analyses of nuts from the same trees but of varying degrees of ripeness.--In order as much as possible to eliminate the variations in the individual nuts, and to discover if those taken from the. same tree would not show greater uniformity in their composition, fifty nuts from one tree near San Ramon were procured for analysis.

Ten of the least ripe among these were analyzed as shown in Series V. All of the individuals of this series were well developed externally, but were full of milk, and not yet sufficiently mature to be picked for making copra.

The ten ripest nuts of the lot were next selected (Series VII). Their husks were of a dead-brown color and thoroughly dry.

The remaining thirty were in a condition which might be termed "fairly ripe"that is, they were of the kind ordinarily used for making copra. Nine of these were analyzed at once (Series VI), and the remainder shipped to Manila for storage and future analysis. In Series V, VI, and VII "total solids" in the milk were determined in addition to the regular analysis. 
Serres V. - Ten muls not fully ripe, frets from tree.

\begin{tabular}{|c|c|c|c|c|c|c|c|c|c|c|c|c|c|}
\hline \multirow{2}{*}{ No. } & \multirow{2}{*}{ 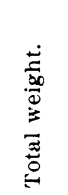 } & \multicolumn{2}{|c|}{ Husk. } & \multicolumn{2}{|c|}{$\begin{array}{l}\text { Nut minus } \\
\text { husk. }\end{array}$} & \multicolumn{2}{|c|}{ Shell. } & \multicolumn{4}{|c|}{ Meat. } & \multicolumn{2}{|c|}{$\begin{array}{l}\text { Copra (HIn- } \\
\text { hydrous). }\end{array}$} \\
\hline & & 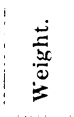 & 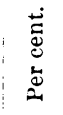 & 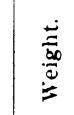 & 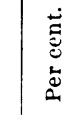 & 苞 & 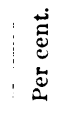 & 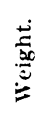 & 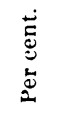 & 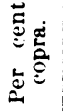 & 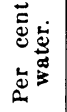 & $\begin{array}{l}\stackrel{2}{\frac{1}{20}} \\
\dot{3}\end{array}$ & 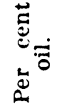 \\
\hline 1 _..... & 4,227 & 2,995 & 70.9 & 1,232 & 29. & 232 & 5.5 & 410 & 9.7 & 33.2 & 66.8 & $13 \mathrm{i}$ & \\
\hline 2 & 4,018 & 2,770 & 69.0 & 1,248 & 31. & 240 & 6.0 & 428 & 10.6 & 34.3 & 65.7 & 147 & (bit. 9 \\
\hline $3 \ldots$ & 4,012 & 2,882 & 71.9 & 1,130 & 28. & 220 & 5.5 & 378 & 9.4 & 30.0 & 70.0 & 114 & 63.9 \\
\hline 4 & 4,535 & 3,380 & 74.5 & $1,1 \%$ & 25. & 219 & 4.8 & 394 & 8.7 & 27.7 & 72.3 & 109 & (i4. 5 \\
\hline 5 -.-.-.- & 3,737 & 2,655 & 71.0 & $1,08:$ & 29. & 205 & 5.5 & 362 & 9.7 & 26.0 & 74.0 & 94 & 56.0 \\
\hline 6 --- & 3,931 & 2,787 & 70.9 & 1,14 & 29. & 214 & 5.5 & 418 & 10.6 & 33.2 & 66.8 & 139 & 62.0 \\
\hline $7 \ldots$ & 3,919 & 2,850 & 72.7 & 1,069 & 27 & 212 & 5.4 & 347 & 8.9 & 29.8 & 70.2 & 103 & 64.6 \\
\hline $8=$ & 3,967 & 2,700 & 68.1 & 1,267 & 31. & 244 & 6.1 & 419 & 10.6 & 37.2 & 62.8 & $15 x$ & titi. 9 \\
\hline 9 & 4,046 & 2,806 & 69.4 & 1,240 & 30. & 239 & 5.9 & 450 & 11.1 & 37.5 & 62.5 & 152 & 65.8 \\
\hline $10 \ldots$ & 3,187 & $.1,965$ & 61.7 & 1,22 & 38. & 240 & 7.5 & 445 & 14.0 & 48.2 & 51.8 & 214 & 69.9 \\
\hline Average & 3,958 & 2,779 & 70.0 & 1,179 & $\begin{array}{l:l}9 & 30 .\end{array}$ & 227 & 5.8 & 405 & 10.3 & 33.7 & 66.3 & 136 & 64.5 \\
\hline \multirow[b]{2}{*}{ No. } & \multicolumn{2}{|c|}{$\begin{array}{l}\text { Copra (an- } \\
\text { hydrous). }\end{array}$} & \multicolumn{4}{|c|}{ Milk. } & \multicolumn{2}{|c|}{ Oil. } & \multicolumn{5}{|c|}{$\begin{array}{l}\text { Calculated to per cent in } \\
\text { nut free from husk. }\end{array}$} \\
\hline & 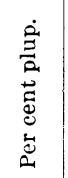 & 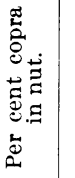 & 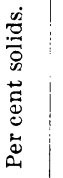 & 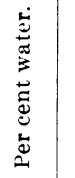 & 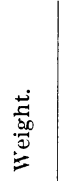 & 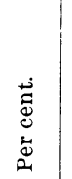 & 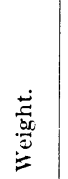 & 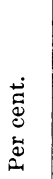 & $\frac{\dot{\bar{d}}}{\frac{\vec{d}}{U}}$ & 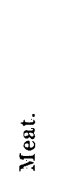 & 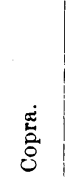 & 总 & $\dot{\bar{c}}$ \\
\hline $1 \ldots$ & 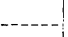 & 3.2 & 6.0 & 94.0 & 590 & 13.9 & - & & 18.8 & 33.3 & 11.0 & 47.9 & \\
\hline $2 \ldots$ & 33.1 & 3.7 & 5.9 & 94.1 & 580 & 14.4 & 98 & 2.4 & 19.2 & 34.3 & 11.8 & 46.5 & 7.9 \\
\hline $3 \ldots$ & 36.1 & 2.8 & 6.5 & 93.5 & 532 & 13.2 & 73 & 1.8 & 19.5 & 33.4 & 10.1 & 47.1 & 6.4 \\
\hline $4 \ldots$ & 35.5 & 2.4 & 6.5 & 93.5 & 542 & 12.0 & 70 & 1.6 & 19.0 & 34.1 & 9.4 & 46.9 & 6.1 \\
\hline $5 \ldots$ & 44.0 & 2.5 & 6.7 & 93.3 & 515 & 13.8 & 53 & 1.4 & 18.9 & 33.5 & 8.7 & 47.6 & 4.9 \\
\hline $6 \ldots$ & 38.0 & 3.5 & 6.0 & 94.0 & 512 & 13.0 & 86 & 2.2 & 18.7 & 36.5 & 12.2 & 44.8 & 7.5 \\
\hline $7_{---}$ & 35.4 & 2.6 & 6.6 & 94.4 & 510 & 13.0 & 67 & 1.7 & 19.8 & 32.6 & 9.6 & 47.7 & 6.2 \\
\hline $8--$ & 33.1 & 3.9 & 6.7 & 93.3 & 604 & 15.2 & 104 & 2.6 & 19.3 & 33.1 & 12.3 & 47.6 & 8.1 \\
\hline 9 & 34.2 & 3.8 & 6.0 & 94.0 & 5.51 & 13.6 & 100 & 2.5 & 19. 3 & 36.3 & 12.3 & 44.4 & 8.1 \\
\hline 10 & 30.1 & 6.7 & 5.6 & 94.4 & 537 & 16.8 & 1.50 & 4.7 & 19.6 & 36.4 & 17.5 & 44.0 & 12.2 \\
\hline A verage & 35.5 & 3.5 & 6.3 & 93.7 & 547 & 13.9 & 89 & 2.3 & 19.2 & 34.4 & 11.5 & 46.4 & 7.5 \\
\hline
\end{tabular}


SERIES VI.-Nine nuts from same tree as Series V, but fairly ripe.

\begin{tabular}{|c|c|c|c|c|c|c|c|c|c|c|c|c|c|}
\hline \multirow[b]{2}{*}{ No. } & \multirow{2}{*}{ 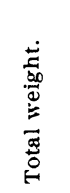 } & \multicolumn{2}{|c|}{ Husk. } & \multicolumn{2}{|c|}{$\begin{array}{l}\text { Nut minus } \\
\text { husk. }\end{array}$} & us & Shell. & \multicolumn{4}{|c|}{ Meat. } & \multicolumn{2}{|c|}{$\begin{array}{l}\text { Copra (an- } \\
\text { hydrous). }\end{array}$} \\
\hline & & $\begin{array}{l}\stackrel{ \pm}{0} \\
\stackrel{00}{0} \\
\dot{0}\end{array}$ & 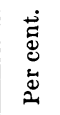 & 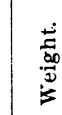 & & 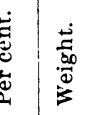 & 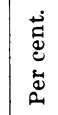 & $\begin{array}{l}\stackrel{2}{0} \\
\stackrel{00}{00} \\
\dot{0}\end{array}$ & 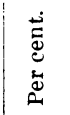 & 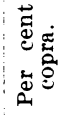 & 䓌岕 & 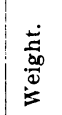 & 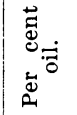 \\
\hline $1 a_{-}$ & 1,644 & 602 & 36.6 & 1,0 & 63 & 207 & 12.6 & 460 & 28.0 & 50.7 & 49.3 & 233 & 63.8 \\
\hline $2^{a}-$ & 1,670 & 650 & 38.9 & 1,0 & 61 & 201 & 12.0 & 455 & 27.3 & 54.6 & 45.4 & 248 & 62.7 \\
\hline $3^{b}-$ & 2,300 & 1,115 & 48.4 & 1,18 & & 229 & 10.0 & 506 & 22.0 & 51.8 & 48.2 & 262 & 64.3 \\
\hline $4^{b} \ldots$ & 2,164 & 1,075 & 49.7 & 1,0 & & 217 & 10.0 & 465 & 21.4 & 50.5 & $49 . \overline{7}$ & 235 & 64.1 \\
\hline $5^{c}-$ & 2,519 & 1,294 & 51.4 & 1,2 & & 231 & 9.1 & 501 & 19.9 & 49.5 & 50.5 & 248 & 64.1 \\
\hline $6^{\mathrm{c}}-\cdots$ & 1,948 & 992 & 50.9 & 9 & & 186 & 9.6 & 400 & 20.5 & 53.6 & 46.4 & 215 & 65.8 \\
\hline $7^{\circ}-\ldots$ & 3,467 & 2,262 & 65.2 & 1,2 & & 232 & 6.7 & 473 & 13.7 & 45.5 & 54.5 & 215 & 66.9 \\
\hline $8 \mathrm{e}-\ldots$ & 2,512 & 1,440 & 57.3 & $1,0^{\prime}$ & & 197 & 7.9 & 450 & 17.9 & 50.4 & 49.6 & 227 & 64.9 \\
\hline $9 \cdot \ldots$ & 3,230 & 1,985 & 61.4 & 1,2 & & 229 & 7.1 & 471 & 14.6 & 44.3 & 55.7 & 208 & 65.7 \\
\hline Average - & 2,384 & 1,268 & 51.1 & 1,1 & & 214 & 9.4 & 465 & 20.6 & 50.1 & 49.9 & 232 & 64.7 \\
\hline \multirow[b]{2}{*}{ No. } & \multicolumn{2}{|c|}{$\begin{array}{l}\text { Copra (an- } \\
\text { hydrous). }\end{array}$} & \multicolumn{4}{|c|}{ Milk. } & \multicolumn{2}{|c|}{ Oil. } & \multicolumn{5}{|c|}{$\begin{array}{l}\text { Calculated to per cent in } \\
\text { nut free from husk. }\end{array}$} \\
\hline & 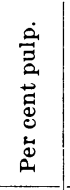 & 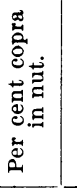 & 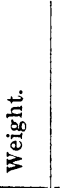 & 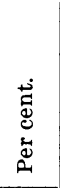 & 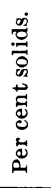 & 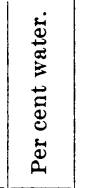 & $\frac{\dot{a}}{\stackrel{0}{0}}$ & 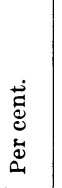 & 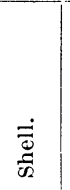 & 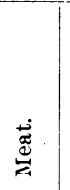 & ঠ் & ن & $\dot{\bar{\sigma}}$ \\
\hline $1 \ldots$ & 36.2 & 14.2 & 375 & 22.8 & 4.4 & d95.6 & 149 & 9.0 & 19.9 & 44.1 & 22.4 & 36.0 & 14.3 \\
\hline 2 & 37.3 & 14.9 & 364 & 21.8 & 4.1 & d 95.9 & 156 & 9.3 & 19.7 & 44.6 & 24.3 & 35.7 & 15.3 \\
\hline $3 \ldots$ & 35.7 & 11.4 & 450 & 19.6 & 5.0 & 95.0 & 169 & 7.3 & 19.3 & 42.7 & 22.1 & 38.0 & 14.2 \\
\hline $4 \ldots$ & 35.9 & 10.8 & 407 & 18.8 & 4.9 & 95.1 & 151 & 7.0 & 19.9 & 42.7 & 21.5 & 37.4 & 13.8 \\
\hline $5 \ldots$ & 35.9 & 9.8 & 493 & 19.6 & 5.7 & 94.3 & 159 & 6.3 & 18.9 & 40.9 & 20.3 & 40.2 & 13.0 \\
\hline - & 34.2 & 11.0 & 370 & 19.0 & 5.2 & 94.8 & 142 & 7.3 & 19.5 & 41.8 & 22.5 & 38.7 & 14.8 \\
\hline 7 - & 33.1 & 6.2 & 500 & 14.4 & 5.6 & 94.4 & 144 & 4.1 & 19.3 & 39.2 & 17.8 & 41.5 & 11.9 \\
\hline 8 - & 35.1 & 9.0 & 425 & 16.9 & 5.8 & 94.2 & 147 & 5.9 & 18.4 & 42.0 & 21.2 & 39.6 & 13.7 \\
\hline 9 & 34.3 & 6.4 & 545 & 16.9 & 6.0 & 94.0 & 137 & 4.2 & 18.4 & 37.8 & 16.7 & 43.8 & 11.0 \\
\hline Average - & 35.3 & 10.4 & 437 & $18: 9$ & 5.2 & 94.8 & 150 & 6.7 & 19.3 & 41.7 & 21.0 & 39.0 & 13.6 \\
\hline
\end{tabular}


Series VII.-Nits from same tree as Servies I, mut dead ripe.

\begin{tabular}{|c|c|c|c|c|c|c|c|c|c|c|c|c|c|}
\hline \multirow[b]{2}{*}{ No. } & \multirow{2}{*}{ 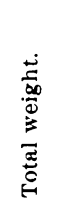 } & \multicolumn{2}{|c|}{ Husk. } & \multicolumn{2}{|c|}{$\begin{array}{c}\text { Nut minus } \\
\text { husk. }\end{array}$} & \multicolumn{2}{|c|}{ Shell. } & \multicolumn{4}{|c|}{ Ment. } & \multicolumn{2}{|c|}{$\begin{array}{l}\text { Copra (an- } \\
\text { hydmus). }\end{array}$} \\
\hline & & 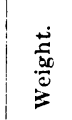 & 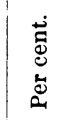 & $\stackrel{\vec{z}}{\overrightarrow{0}}$ & & 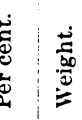 & 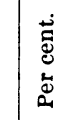 & 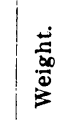 & 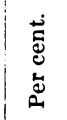 & 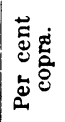 & 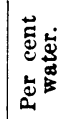 & 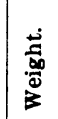 & : \\
\hline $1 \ldots$ & 1,624 & 545 & 33.6 & 1,07 & 66 & 217 & $7 \mid 13.4$ & 462 & 28.4 & 51.9 & 48.1 & 240 & 63.9 \\
\hline 2 & 1,493 & 486 & 32.6 & 1,00 & & 202 & \begin{tabular}{l|l}
2 & 13.5 \\
\end{tabular} & 450 & 30.1 & 55.1 & 44.9 & 248 & 66.0 \\
\hline $3 \ldots$ & 1,427 & 547 & 38.3 & $8 *$ & & 185 & 513.0 & 415 & 29.1 & 58.6 & 41.4 & 243 & 66.6 \\
\hline 4 -- & 1,495 & 445 & 29.8 & 1,05 & & 187 & $7 \mid 12.5$ & 438 & 29.3 & 45.3 & 54.7 & 198 & 65.3 \\
\hline $5-$ & 1,568 & 528 & 33.7 & 1,04 & & $20^{2}$ & 2 12.9 & 441 & 28.1 & 53.5 & 46.5 & 236 & (i4. 2 \\
\hline 6 & 1,437 & 472 & 32.8 & 96 & & 198 & \begin{tabular}{l|l}
8 & 13.8
\end{tabular} & 437 & 30.4 & 56.7 & 43.3 & 248 & 67.1 \\
\hline $7 \ldots$ & 1,716 & 631 & 36.8 & 1,08 & & 202 & \begin{tabular}{l|l}
2 & 11.8
\end{tabular} & 476 & 27.7 & 51.8 & 48.2 & 246 & 67.5 \\
\hline & 1,564 & 489 & 31.3 & 1,07 & & 206 & \begin{tabular}{l|l}
6 & 13.1
\end{tabular} & 483 & 30.9 & 53.3 & 46.7 & 261 & 65.0 \\
\hline 9 & 1,452 & 450 & 31.0 & 1,00 & & 188 & \begin{tabular}{l|l}
8 & 13.0 \\
\end{tabular} & 436 & 30.0 & 53.5 & 46.5 & 233 & 65.6 \\
\hline 10 & 1,806 & 612 & 33.9 & 1,19 & & 219 & 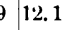 & 496 & 27.5 & 53.6 & 46.4 & 266 & 68.3 \\
\hline Average & 1,558 & 520 & 33.4 & 1,03 & & 6.6 & \begin{tabular}{l|l}
1 & 12.9
\end{tabular} & 453 & 29.1 & 53.3 & 46.7 & 242 & 66.0 \\
\hline \multirow[b]{2}{*}{ No. } & \multicolumn{2}{|c|}{$\begin{array}{l}\text { Copra (an- } \\
\text { hydrous). }\end{array}$} & \multicolumn{4}{|c|}{ Milk. } & \multicolumn{2}{|c|}{ Oil. } & \multicolumn{5}{|c|}{$\begin{array}{l}\text { Calculated to per cent in } \\
\text { nut free from husk. }\end{array}$} \\
\hline & 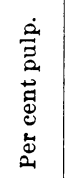 & 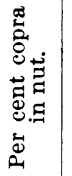 & 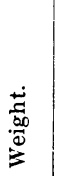 & $\begin{array}{l}\dot{\Xi} \\
\dot{\Xi} \\
\dot{0}\end{array}$ & 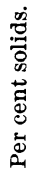 & 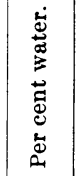 & 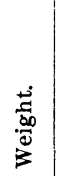 & 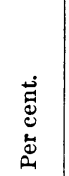 & ॠ & 芯 & $\begin{array}{l}\text { 芯 } \\
\text { 今. } \\
\text {. }\end{array}$ & 兽 & $\ddot{0}$ \\
\hline & 36.1 & 14.8 & 400 & 24.6 & 4.5 & 95.5 & 153 & 9.4 & 20.1 & 42.8 & 22.2 & 37.1 & 14.2 \\
\hline $2 \ldots$ & 34.0 & 16.6 & 355 & 23.8 & 4.3 & 95.7 & 164 & 11.0 & 20.0 & 44.7 & 24.7 & 35.3 & 16.3 \\
\hline $3 \ldots$ & 33.4 & 17.1 & 280 & 19.6 & 5.0 & 95.0 & 162 & 11.3 & 21.0 & 47.2 & 27.6 & 31.8 & 18.4 \\
\hline $4--$ & 34.7 & 13.3 & 425 & 28.4 & 4.3 & 95.7 & 129 & 8.6 & 17.8 & 41.7 & 18.9 & 40.5 & 12.3 \\
\hline $5 \ldots$ & 35.8 & 15.1 & 397 & 25.3 & 4.1 & 95.9 & 152 & 9.7 & 19.4 & 42.4 & 22.7 & 38.2 & 14.6 \\
\hline 6 & 32.9 & 17.2 & 330 & 23.0 & 4.5 & 95.5 & 166 & 11.6 & 20.5 & 45.3 & 25.7 & 34.2 & 17.3 \\
\hline $7 \ldots$ & 32.5 & 14.4 & 407 & 23.7 & 4.0 & 96.0 & 166 & 9.7 & 18.6 & 43.9 & 22.7 & 37.5 & 15.3 \\
\hline $8--$ & 35.0 & 16.7 & 386 & 24.7 & 4.3 & 95.7 & 170 & 10.9 & 19.2 & 44.9 & 24.3 & 35.9 & 15.8 \\
\hline 9 - & 34.4 & 16.1 & 378 & 26.0 & 4.6 & 95.4 & 153 & 10.5 & 18.8 & 43.5 & 23.3 & 37.7 & 15.3 \\
\hline 10 . & 31.7 & 14.7 & 479 & 26.5 & 4.3 & 95.7 & 182 & 10.1 & 18.3 & 41.6 & 22.3 & 40.1 & 15.2 \\
\hline Average & 34.0 & 15.6 & 384 & 24.6 & 4.4 & 95.6 & 160 & 10.3 & 19.4 & 43.8 & 23.4 & 36.8 & 15.5 \\
\hline
\end{tabular}

While there is still some individual variation among nuts from the same tree, these last analyses very conclusively show the change which is taking place as the fruit becomes riper. The average percentages of copra and oil, for example, in the nut free from husk in the green fruit, are only 11.5 and 7.5 , respectively, but they rise to 21 and 13.6 in the "fairly ripe" nuts, and assume a maximum of 23.4 and $\mathbf{1 5 . 5}$ in the case of the series which had been allowed completely to ripen while still on the tree. This gain is partially due to an increase in the percentage of meat, which runs 34.4, 41.\%, and 43.6 in Series V, VI, and VII, respectively, at the expense of milk, which falls from 46.4 to 39 and finally to 36.8 , but it is also largely accounted for by the increase of solid matter and loss of water in the former. The percentage of anhydrous copra in 
the meat of the green fruit is 39.8 ; it rises to 511.1 in that of the "fairly ripe" nuts and increases to 5:3.; in those marked "dead ripe." 'T'he "fairly ripe" muts which had been sent to Manila showed 51.1 pere cent of anhydrous eopra in the meat alter standing during one month, and, after two, 53.9 per cent, this last ligure being very nearly the same as that obtained from the "dead-ripe" nuts taken directly from the tree. 'The amount of oil obtainable from this copra also seems slightly to increase with age, running 64.5, 64.7, and 66 in the three series (V, VI, and VII), and in those nuts which had stood for one and two months it was found to be $6 \% .09$ and $6 \% .11$, respectively. However, it is also quite possible that these changes of oil eontent in the copra in greater part are due to individual variation in the nuts themselves.

Another interesting fact brought out by these analyses is the gradual decrease of the amount of the total solids in the milk as a nut grows riper. In green nuts this quantity averaged 6.3 per cent and the milk has a sweet, pleasant taste and is saturated with a gas which I have proven to be carbon dioxide. 'The occurrence of an alcoholic fermentation in the center of a sound, growing fruit, with absolutely no access of air to the milk inside, is practically impossible, and, besides, analytical tests have proven the absence of alcohol in the fresh milk, so that probably the carbon dioxide is a by-product of a process, possibly due to enzymes, which is constantly changing sugar and water into fat and cellulose. The milk from the nuts called "fairly ripe" was not so pleasant to the taste, contained very little, if any, carbon dioxide, and had decreased in total solids to 5.2 per cent; while the "dead-ripe" samples produced a milk which was rather insipid, which contained no gas, and which in most cases had a few drops of clear oil floating on the surface; the total solids in the latter had been further reduced to 4.4 per cent.

Changes taking place during the ripening of a coconut.-From the foregoing data, and from observations made on very young nuts, the following are probably the changes which a young coconut undergoes before it reaches maturity:

When the young fruit first appears it consists of a white, astringent tasting, semifibrous mass, which afterwards is destined to form the husk; and of a thin, green outer skin. The nut gradually increases in size, with very little change in composition, until it has grown to be about 3 inches in diameter. It then has a comparatively small, hollow space in the center which is completely filled with a watery fluid of an astringent, slightly acid taste, and which is much like the juice from a green husk. As this period begins, a rudimentary shell is formed around the inner surface of the nut; at first this is very thin and soft, but slowly it becomes thicker and harder. Not until the nut has reached its maximum size, with its shell completed, is there any indication of meat or of oily material. When the shell has been formed the milk changes in character, 
it becomes rather sweet, and a slimy, gelatinous mass, having a sweetish taste and containing comparatively little oil begins to deposit on the inside of the former. At first this forms ehielly on the lower half of the nut, hut finally it covers the whole inner surface. 'This pulpy mass soon grows thicker and denser, it increases in oil content at the expense of sugar in the milk, until it assumes the well-known characteristics of ordinary coconut meat. During this last stage the evolution of carbon dioxide which previously was mentioned occurs. Even in ripe nuts, after they have been picked from the tree, there seems to be a slight continuation of the hardening process in the meat, covering a period of from two to three months, or until the sprout makes its appearance. 'Then other changes occur, the reverse of those which had taken place previously; the nourishment concentrated and stored up as fat is now transformed into sugars and other bodies capable of being directly assimilated by the young plant. As this process goes on the embryo or "foot" gradually increases in size until it occupies the whole space inside the nut and makes use of all the nourishment contained therein for the growth of the young tree.

Therefore, for the largest yield of copra and oil, only thoroughly ripe nuts (the husks of which have begun to turn brown) should be used, and it is often advisable to allow the latter to stand in a dry place for a few weeks before they are opened. The greatest care should be taken to avoid using green nuts, as it is shown by the tables given above that a loss of almost 50 per cent may thus result.

On the other hand, coconuts should not be stored too long, for in about three months the embryo begins to grow, and, even before that time, those nuts which may have been cracked or bruised in gathering, have a tendency to become rancid.

Analysis of nuts of different color.-In a certain portion of San Ramon farm there exist, growing side by side in the same kind of soil, two apparently different varieties of coconut trees, one of which uniformly produces nuts of a golden-yellow color, while the other bears a light-green fruit. Both varieties eventually turn brown at maturity. Analyses of these nuts are given in the accompanying tables, Series VIII being ten ripe nuts from a tree which bears a green fruit, while Series IX is made up of nuts from a tree about 50 feet away whose product is yellow until it becomes "dead ripe." • 
Series VIII. - Tom thoroughly ripe muts from ome tree.

[The nuts on this tree all have a green husk until they become "dead ripe," when they change to a dull brown.]

\begin{tabular}{|c|c|c|c|c|c|c|c|c|c|c|c|c|c|}
\hline \multirow[b]{2}{*}{ No. } & \multirow{2}{*}{ 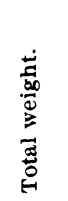 } & \multicolumn{3}{|c|}{ Husk. } & \multicolumn{2}{|c|}{$\begin{array}{l}\text { Nut minus } \\
\text { husk. }\end{array}$} & \multicolumn{3}{|c|}{ Shell. } & \multicolumn{4}{|c|}{ Meat. } \\
\hline & & & 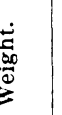 & 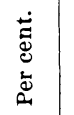 & 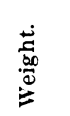 & 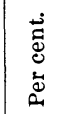 & 递 & & 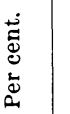 & 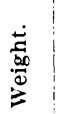 & 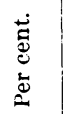 & 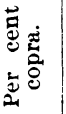 & 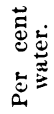 \\
\hline $1-1-1$ & \multicolumn{2}{|c|}{1,490} & 585 & 39.3 & 905 & 60.7 & \multicolumn{2}{|c|}{204} & 13.7 & 397 & 26.6 & 44.6 & in. 4 \\
\hline 2 & \multicolumn{2}{|c|}{2,160} & 640 & 29.6 & 1,520 & 70.4 & \multicolumn{2}{|c|}{275} & 12.4 & 663 & 30.7 & 52.6 & 47.4 \\
\hline $3 \ldots$ & \multicolumn{2}{|c|}{1,632} & 657 & 40.3 & 975 & 59.7 & \multicolumn{2}{|c|}{227} & 13.9 & 428 & 26.2 & 46.3 & 53.7 \\
\hline $4 \ldots$ & \multicolumn{2}{|c|}{1,482} & 437 & 29.5 & 1,045 & 70.5 & \multicolumn{2}{|c|}{235} & 15.9 & 438 & 29.5 & 44.4 & 55.6 \\
\hline $5 \ldots$ & \multicolumn{2}{|c|}{1,862} & 665 & 35.7 & 1,197 & 64.3 & \multicolumn{2}{|c|}{265} & 14.2 & 482 & 25.9 & 44.4 & 55.7 \\
\hline 6 & \multicolumn{2}{|c|}{1,517} & 395 & 26.0 & 1,122 & 74.0 & \multicolumn{2}{|c|}{255} & 16.8 & 467 & 30.8 & 53.5 & $4(i .5)$ \\
\hline $7 \ldots$ & \multicolumn{2}{|c|}{1,702} & 567 & 33.3 & 1,135 & 66.7 & \multicolumn{2}{|c|}{260} & 15.3 & 498 & 29.2 & 46.8 & 53.2 \\
\hline $8-\ldots-1$ & \multicolumn{2}{|c|}{1,623} & 520 & 32.0 & 1,103 & 68.0 & \multicolumn{2}{|c|}{$26 \overline{5}$} & 16.4 & 476 & 29.3 & 51.3 & $48.1 i$ \\
\hline 9 - & 1,9 & & 700 & 36.8 & 1,200 & 63.2 & 27 & 70 & 14.2 & 505 & 26.6 & 44.6 & 55.3 \\
\hline 10 & 1,6 & & 475 & 28.4 & 1,198 & 71.6 & 21 & 10 & 12.6 & 521 & 31.1 & 57.0 & 43.0 \\
\hline Average --.- & 1,7 & & 564 & 33.1 & 1,140 & 66.9 & 24 & 47 & 14.6 & 488 & 28.6 & 48.5 & 51.5 \\
\hline & CoI & ra (ar & nhydr & ous). & Mil & lk. & $\mathrm{Oi}$ & il. & & $\begin{array}{l}\text { alculate } \\
\text { nut fr }\end{array}$ & $\begin{array}{l}\text { ed to } \\
\text { ree fro }\end{array}$ & $\begin{array}{l}\text { reen } \\
\text { husl }\end{array}$ & \\
\hline No. & 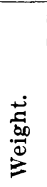 & 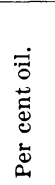 & 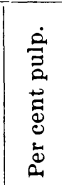 & 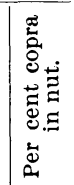 & 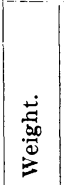 & 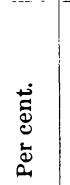 & 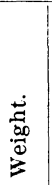 & 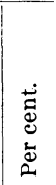 & 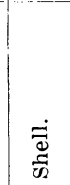 & 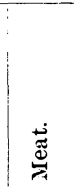 & 芯 & 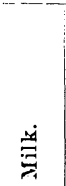 & $\dot{\vec{\sigma}}$ \\
\hline $1 \ldots$ & 177 & 64.2 & 35.8 & 11.9 & 304 & 20.4 & 114 & 7.6 & 22.5 & 43.9 & 19.6 & 33.6 & 12.6 \\
\hline $2 \ldots$ & 349 & 62.5 & 37.5 & 16.1 & 582 & 26.9 & 218 & 10.1 & 18.1 & 43.6 & 23.0 & 38.3 & 14.3 \\
\hline $3 \ldots$ & 198 & 62.7 & 37.3 & 12.1 & 320 & 19.6 & 124 & 7.6 & 23.3 & 43.9 & 16.1 & 32.8 & 12.7 \\
\hline 4 & 195 & 62.6 & 37.4 & 13.1 & 372 & 25.1 & 122 & 8.2 & 22.5 & 41.9 & 18.7 & 35.6 & 11.7 \\
\hline 5 - & 214 & 60.8 & 39.2 & 11.5 & 450 & 24.2 & 130 & 7.0 & 22.1 & 40.3 & 17.9 & 37.6 & 10.9 \\
\hline 6 - & 250 & 65.0 & 35.0 & 16.5 & 390 & 25.7 & 163 & 10.7 & 22.7 & 41.6 & 22.3 & 34.8 & 14.5 \\
\hline 7 - & 233 & 67.0 & 33.0 & 13.7 & 377 & 22.2 & 170 & 9.2 & 22.9 & 43.9 & 20.5 & 33.2 & 13.8 \\
\hline 8 - & 242 & 64.3 & 35.7 & 14.9 & 362 & 22.3 & 156 & 9.6 & 24.0 & 43.2 & 22.0 & 32.8 & 14.1 \\
\hline 9 & 225 & 65.9 & 34.1 & 11.9 & 425 & 22.4 & 148 & 7.8 & 22.5 & 42.1 & 18.8 & 35.4 & 12.4 \\
\hline 10 & 297 & 64.6 & 35.4 & 17.7 & 467 & 27.9 & 192 & 11.5 & 17.5 & 43.5 & 24.8 & 39.0 & 16.0 \\
\hline Average & 238 & 64.0 & 36.0 & 13.9 & $\overline{405}$ & 23.7 & 154 & 8.9 & 21.8 & 42.8 & 20.4 & 35.3 & 13.3 \\
\hline
\end{tabular}


SERIES IX. - Tin thorough!! ripe muts from one tree.

[These nuts have a golden-yellow color until dead ripe, when they look like those of Series VIII.]

\begin{tabular}{|c|c|c|c|c|c|c|c|c|c|c|c|c|c|}
\hline \multirow[b]{2}{*}{ No. } & \multirow{2}{*}{\multicolumn{2}{|c|}{ 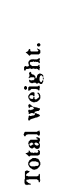 }} & \multicolumn{2}{|c|}{ Husk. } & \multicolumn{2}{|c|}{$\begin{array}{l}\text { Nut minus } \\
\text { husk. }\end{array}$} & \multicolumn{3}{|c|}{ Shell. } & \multicolumn{4}{|c|}{ Mert. } \\
\hline & & & 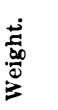 & 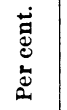 & 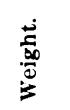 & 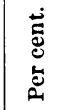 & 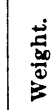 & & 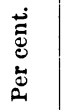 & 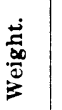 & 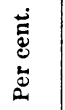 & 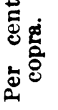 & 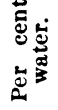 \\
\hline 1 & \multicolumn{2}{|c|}{1.613} & 428 & 26.5 & 1,185 & 73.5 & 23 & & 14.3 & 495 & 30.7 & 47.1 & 52.9 \\
\hline $2 \ldots$ & \multicolumn{2}{|c|}{1.960} & 520 & 26.7 & 1,440 & 73. 3 & 25 & & 13.0 & 589 & 30.0 & 53.6 & 46.4 \\
\hline $3 \ldots$ & \multicolumn{2}{|c|}{1,657} & 375 & 22.6 & 1,282 & 77.3 & 24 & 10 & 14.5 & 547 & 33.0 & 53.4 & 46.6 \\
\hline $4 \ldots$ & \multicolumn{2}{|c|}{1,608} & 353 & 22.0 & 1,255 & 78.0 & 23 & 35 & 14.5 & 545 & 33.9 & 52.7 & 49.3 \\
\hline 5 & \multicolumn{2}{|c|}{1,653} & 335 & 20.3 & 1,318 & 79. 7 & 24 & 45 & 14.8 & 568 & 34.4 & 53.1 & 46.9 \\
\hline $6 \ldots$ & \multicolumn{2}{|c|}{1,577} & 560 & 35.5 & 1,017 & 64.5 & 20 & 05 & 13.0 & 432 & 27.4 & 49.6 & 51.3 \\
\hline $7 \ldots$ & \multicolumn{2}{|c|}{1,780} & 510 & 28.7 & 1,270 & 71.3 & 25 & 52 & 14.2 & 533 & 29.9 & 53.6 & 46.4 \\
\hline $8 \ldots$ & \multicolumn{2}{|c|}{1,650} & 380 & 23.0 & 1,270 & 77.0 & 24 & 47 & 15.0 & 538 & 32.6 & 51.2 & 48.8 \\
\hline 9 & \multicolumn{2}{|c|}{1,597} & 497 & 31.1 & 1,100 & 68.9 & 22 & 25 & 14.2 & 495 & 30.9 & 43.4 & 56.6 \\
\hline 10 & \multicolumn{2}{|c|}{1,926} & 486 & 25.2 & 1,440 & 74.8 & 25 & 57 & 13.4 & 591 & 30.7 & 51.2 & 48.8 \\
\hline Average --- & \multicolumn{2}{|c|}{1,702} & 444 & 26.2 & 1,258 & 73.8 & 23 & 39 & 14.1 & 533 & 31.3 & 50.9 & 49.1 \\
\hline \multirow[b]{2}{*}{ No. } & \multicolumn{4}{|c|}{ Copra (anhydrous). } & \multicolumn{2}{|c|}{ Milk. } & \multicolumn{2}{|c|}{ Oil. } & \multicolumn{5}{|c|}{$\begin{array}{l}\text { Calculated to per cent in } \\
\text { nut free from husk. }\end{array}$} \\
\hline & 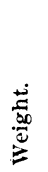 & 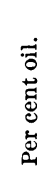 & 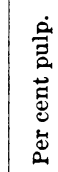 & 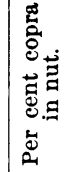 & 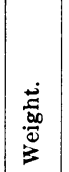 & 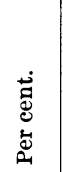 & 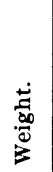 & 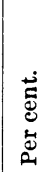 & $\frac{\dot{\vec{d}}}{\ddot{\not}}$ & 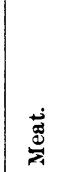 & 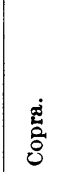 & $\dot{J}$ & $\dot{0}$ \\
\hline $1 \ldots$ & 233 & \multirow{10}{*}{\multicolumn{2}{|c|}{ 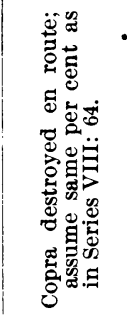 }} & 14.5 & 460 & 28.5 & 149 & 9.2 & 19.4 & 41.8 & 19.6 & 38.8 & 12.6 \\
\hline $2 \ldots$ & 315 & & & 16.1 & 595 & 30.3 & 202 & 10.3 & 17.8 & 40.9 & 21.9 & 41.3 & 14.0 \\
\hline $3 \ldots$ & 292 & & & 17.6 & 495 & 29.8 & 187 & 11.3 & 18. 7 & 42.7 & 22.8 & 38.6 & 14.6 \\
\hline $4 \ldots$ & 287 & & & 17.8 & 475 & 29.6 & 184 & 11.4 & 18.7 & 43.4 & 22.9 & 37.9 & 14.6 \\
\hline $5 \ldots$ & 301 & & & 18.2 & 505 & 30.5 & 193 & 11.7 & 18.6 & 43. 1 & 22.8 & 38.3 & 14.6 \\
\hline $6 \ldots$ & 214 & & & 13.6 & 380 & 24.1 & 137 & 8.7 & 20.1 & 42.5 & 21.1 & 37.4 & 13.5 \\
\hline 7 & 285 & & & 16.0 & 485 & 27.2 & 182 & 10.2 & 19.9 & 42.0 & 22.4 & 38.2 & 14.4 \\
\hline $8 \ldots$ & 276 & & & 16.7 & 485 & 29.4 & 177 & 10.7 & 19.5 & 42.9 & 21.7 & 38.2 & 13.9 \\
\hline $9 \ldots$ & 215 & & & 13.5 & 380 & 23.8 & 138 & 8.6 & 20.5 & 45. 0 & 19.5 & 34.5 & 12.5 \\
\hline 10 & 302 & & & 15.7 & 592 & 30.7 & 193 & 10.0 & 17.9 & 41.0 & 21.0 & 41.1 & 13.4 \\
\hline Average -..- & 272 & & - & 16.0 & 485 & 28.4 & 174 & 10.2 & 19.1 & 42.5 & 21.6 & 38.4 & 13.8 \\
\hline
\end{tabular}


Very little difference can be olserved hetween these two varieties; the average weiglit is almost exactly the same; the perentage of husk and shell is somewhat lower in the yellow nuts, but this andvantage to a large extent is counterbalanced by their percentage in milk, se that the annount of meat in the two remains practically the same. 'The yellow nuts average 27\% grams of anhydrous copra against 238 grams in the green ones, which is quite decidedly in favor of the former.

Unfortunately, the copra from Series IX was spoiled in transit to Manila. Calculations on the oil contents of this series were therefore based on the assumption that this copra would have contained 64 per cent oil-that is, the same percentage as that found in Series VIII. Figuring the yield of oil on this basis, we have an average of 174 grams for the yellow nuts against $\mathbf{1 5 4}$ for the green ones. However, it will be noticed that these tables show a difference of over 100 grams in each series between the maximum and minimum weight of oil, therefore if :nother series of analyses of nuts from these two trees were to be made possibly the slight advantage in favor of the yellow nuts might be reversed. At any rate it may be concluded that the color of a nut has very little, if any, influence on its composition.

Nuts from different localities.-In order to test the truth of the statement that coconuts produced by trees growing along the seashore are of a quality superior to those taken from farther inland, ten nuts were selected at random from a large pile gathered near the sea and analyzed as shown in the accompanying Series $X$, while a like number was secured from a similar one containing the product of trees growing some 1,800 feet inland (Series XI). 


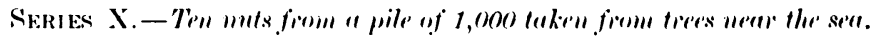

\begin{tabular}{|c|c|c|c|c|c|c|c|c|c|c|c|c|c|}
\hline \multirow[b]{2}{*}{ No. } & \multirow{2}{*}{ 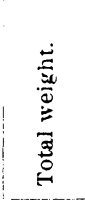 } & & \multicolumn{2}{|c|}{ Husk. } & \multicolumn{2}{|c|}{$\begin{array}{l}\text { Nut minus } \\
\text { husk. }\end{array}$} & \multicolumn{3}{|c|}{ Shell. } & \multicolumn{4}{|c|}{ Mert. } \\
\hline & & & $\begin{array}{l}\frac{3}{5} \\
\frac{30}{3} \\
3\end{array}$ & 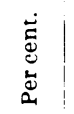 & $\begin{array}{l}\frac{+}{2} \\
\frac{\infty}{0} \\
\frac{1}{5}\end{array}$ & 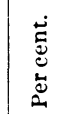 & $\stackrel{+}{0}$ & & 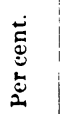 & $\begin{array}{l}\frac{\pi}{\infty} \\
\frac{0}{0} \\
3\end{array}$ & 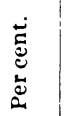 & 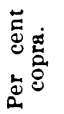 & 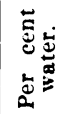 \\
\hline $1^{a}$ & \multicolumn{2}{|c|}{3,125} & 1,420 & 45.4 & 1,705 & 54.6 & 31 & 10 & 9.9 & 745 & 23.8 & 54.3 & 45.7 \\
\hline $2^{11}-$ & \multicolumn{2}{|c|}{2,165} & (633 & 29.2 & 1,532 & 70.8 & $2 \varepsilon$ & 85) & 13.2 & ii 27 & 29.0 & 59.1 & 40.9 \\
\hline $3 c^{c}$ & \multicolumn{2}{|c|}{2,520} & 1,210 & 48.0 & 1,310 & 52.0 & 31 & 01 & 12.0 & 549 & 21.8 & 54.8 & 45.2 \\
\hline $4^{\prime \prime}$ & \multicolumn{2}{|c|}{$3,49 \cdot 2$} & $1,5,50$ & 44.7 & 1,932 & 55.3 & 4 & 25 & 12.1 & 775 & 2.2 & 45.0 & 55.0 \\
\hline 5$)^{a}-$ & \multicolumn{2}{|c|}{2,292} & 992 & 43.3 & 1,300 & 5 (f). 7 & 30 & 10 & 13.1 & 608 & 26.5 & 57.8 & 42.2 \\
\hline $6^{4}$ & \multicolumn{2}{|c|}{3,215} & 1,355 & 42.1 & 1,860 & 57.9 & 3 & 59 & 11.2 & 701 & 21.8 & 49.8 & 50.2 \\
\hline 71. & \multicolumn{2}{|c|}{$2,78 \pi$} & 1,405 & 50.4 & 1,380 & 49.6 & $2 !$ & 91 & 10.5 & 594 & 21.3 & 42.2 & 57.8 \\
\hline $8^{\prime \prime}$ & \multicolumn{2}{|c|}{2,512} & 792 & 31.5 & 1,720 & 68.5 & 3 & 40 & 13.5 & 738 & 29.4 & 57.3 & 42.7 \\
\hline $9^{\mathrm{e}}$ & \multicolumn{2}{|c|}{3,240} & 1,320 & 40.8 & 1,920 & 59.2 & 3 & 80 & 11.7 & 780 & 24.1 & 51.5 & 48.5 \\
\hline $10^{4}$ & \multicolumn{2}{|c|}{2,765} & 1,170 & 42.3 & 1,595 & 57.7 & 20 & 62 & 9.5 & 683 & 24.7 & 49.7 & 50.3 \\
\hline Average ... & \multicolumn{2}{|c|}{2,811} & 1,186 & 41.8 & 1,625 & 58.2 & 3 & 25 & 11.7 & 680 & 24.4 & 52.2 & 47.8 \\
\hline \multirow[b]{2}{*}{ No. } & \multicolumn{4}{|c|}{ Copra (anhydrous). } & \multicolumn{2}{|c|}{ Milk. } & \multicolumn{2}{|c|}{ Oil. } & \multicolumn{5}{|c|}{$\begin{array}{l}\text { Calculated to per cent in } \\
\text { nut free from husk. }\end{array}$} \\
\hline & 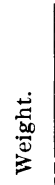 & 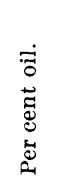 & 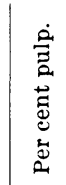 & 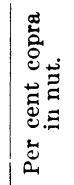 & 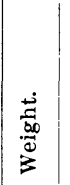 & 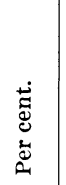 & 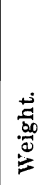 & 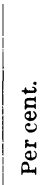 & $\frac{\dot{0}}{\tilde{d}}$ & 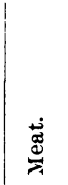 & 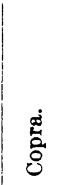 & $\underset{\Xi}{ٍ}$ & $\dot{\bar{\sigma}}$ \\
\hline $1 \ldots$ & 404 & 65.3 & 34.7 & 12.9 & $6 \overline{0} 0$ & 20.9 & 264 & 8.4 & 18.2 & 43.7 & 23.7 & 38.1 & 15.5 \\
\hline 2 & 370 & 67.3 & 32.7 & 17.1 & 620 & 28.6 & 249 & 11.5 & 18.6 & 40.9 & 24.2 & 40.5 & 16.3 \\
\hline $3 \ldots$ & 306 & 65.7 & 34.3 & 12.1 & 460 & 18. 2 & 201 & 8.0 & 23.0 & 41.9 & 23.4 & 35.1 & 15.4 \\
\hline $4 \ldots$ & 349 & 64.0 & 36.0 & 10.0 & 732 & 21.0 & 224 & 6.4 & 22.0 & 40.1 & 18.1 & 37.9 & 11.6 \\
\hline $5 \ldots$ & 349 & 69.8 & 30.2 & 15.2 & 392 & 17.1 & 244 & 10.1 & 23.1 & 46.8 & 26.8 & 30.1 & 18.7 \\
\hline 6 & 349 & $-\ldots-$ & $--\ldots$ & 10.9 & 800 & 24.9 & $\ldots$ & $\ldots$ & 19.3 & 37.7 & 18.8 & 43.0 & - \\
\hline 7 & 251 & 61.6 & 38.4 & 9.0 & 495 & 17. 8 & 155 & 5.6 & 21.1 & 43.0 & 18.2 & 35.9 & 11.2 \\
\hline 8 & 423 & 63.6 & 36.4 & 16.8 & 642 & 25.6 & 269 & 10.7 & 19.8 & 42.9 & 24.6 & 37.3 & 15.6 \\
\hline $9 \ldots$ & 402 & 62.8 & 37.2 & 12,4 & 760 & 23.4 & 253 & 9.5 & 19.8 & 40.6 & 20.9 & 39.6 & 13.2 \\
\hline 10 & 339 & $--\cdots$ & $\ldots$ & 12.3 & 650 & 23.5 & --- & $-\cdots$ & 16.4 & 42.8 & 21.3 & 40.8 & $-\cdots-$ \\
\hline Average....-. & 354 & 65.0 & 35.0 & 12.9 & 620 & 22.1 & 232 & 8.8 & 20.1 & 42.1 & 22.0 & 37.8 & 14.7 \\
\hline
\end{tabular}


SERIEs XI.-Ten muts from a pile of 1,000 taken from trees imlam alomit 1,800 fert.

\begin{tabular}{|c|c|c|c|c|c|c|c|c|c|c|c|c|c|}
\hline \multirow[b]{2}{*}{ No. } & \multirow{2}{*}{ 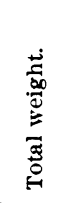 } & \multicolumn{3}{|c|}{ Husk. } & \multicolumn{2}{|c|}{$\begin{array}{l}\text { Nut minus } \\
\text { husk. }\end{array}$} & \multicolumn{3}{|c|}{ shell. } & \multicolumn{4}{|c|}{ IIeat. } \\
\hline & & & $\begin{array}{l}\dot{3} \\
\frac{\pi}{200} \\
\overrightarrow{2} \\
3\end{array}$ & 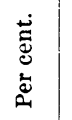 & 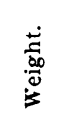 & 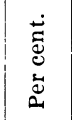 & 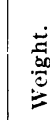 & & 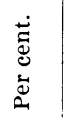 & 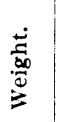 & $\begin{array}{l}\stackrel{\stackrel{\Xi}{\Xi}}{0} \\
\stackrel{\Delta}{\Delta}\end{array}$ & 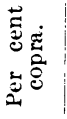 & 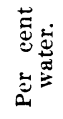 \\
\hline $1^{2}-\ldots$ & \multirow{2}{*}{\multicolumn{2}{|c|}{$\begin{array}{l}4,114 \\
2,500\end{array}$}} & 1,612 & 39.2 & 2,502 & 60.8 & 54 & 40 & 13.1 & 857 & 20.8 & 45.3 & 54.7 \\
\hline $2^{b}-\ldots$ & & & 865 & 34.6 & 1,635 & 65.4 & 35 & 2 & 14.1 & 726 & 29.0 & 50.9 & 49.1 \\
\hline $3^{b}-\ldots$ & \multicolumn{2}{|c|}{2,512} & 652 & 26.0 & 1,860 & 74.0 & 35 & 2 & 14.0 & 746 & 29.7 & 57.0 & 43.0 \\
\hline $4^{b}-$ & \multicolumn{2}{|c|}{2,763} & 863 & 31.2 & 1,900 & 68.8 & 33 & 37 & 12.2 & 766 & 27.7 & 45.4 & 54.6 \\
\hline $55^{\mathrm{b}} \ldots$ & \multicolumn{2}{|c|}{2,745} & 815 & 29.7 & 1,930 & 70.3 & 34 & 43 & 12.5 & 752 & 27.4 & 51.2 & 48.8 \\
\hline $6^{c}-\ldots$ & \multicolumn{2}{|c|}{4,102} & 1,592 & 38.8 & 2,510 & 61.2 & 45 & 22 & 11.0 & 911 & 22.2 & 45.5 & 54.5 \\
\hline $7^{\mathrm{b}}-\ldots$ & \multicolumn{2}{|c|}{$2,48 \pi$} & 525 & 21.1 & 1,960 & 78.9 & 34 & 45 & 13.9 & 820 & 33.0 & 49.3 & 50.7 \\
\hline $8^{b}-$ & \multicolumn{2}{|c|}{1,423} & 425 & 29.9 & 998 & 70.1 & 23 & 35 & 16.5 & 473 & 33.2 & 59.3 & 40.7 \\
\hline $9^{a}$ & \multirow{2}{*}{\multicolumn{2}{|c|}{$\begin{array}{l}2,675 \\
3,620\end{array}$}} & 1,000 & 37.4 & 1,675 & 62.6 & 34 & 42 & 12.8 & 603 & 22.5 & 37.3 & 62.7 \\
\hline $10^{4}$ & & & 1,170 & 32.3 & 2,450 & 67.7 & 43 & 39 & 12.1 & 936 & 25.9 & 49.3 & 50.7 \\
\hline Average & \multicolumn{2}{|c|}{2,894} & 952 & 32.0 & 1,942 & 68.0 & 37 & 74 & 13.2 & 759 & 27.2 & 49.1 & 50.9 \\
\hline \multirow[b]{2}{*}{ No. } & \multicolumn{4}{|c|}{ Copra (anhydrous). } & \multicolumn{2}{|c|}{ Milk. } & \multicolumn{2}{|c|}{ Oil. } & \multicolumn{5}{|c|}{$\begin{array}{l}\text { Calculated to per cent in } \\
\text { nut free from husk. }\end{array}$} \\
\hline & 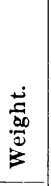 & 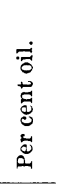 & 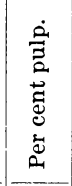 & 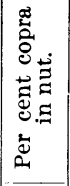 & 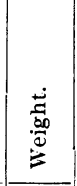 & 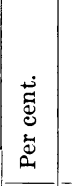 & 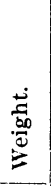 & 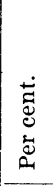 & 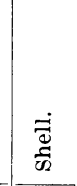 & 芯 & 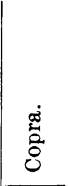 & ن & ந் \\
\hline $1 \ldots$ & 388 & 57.7 & 42.3 & 9.4 & 1,105 & 26.9 & 224 & 5.4 & 21.6 & 34.2 & 15.5 & 44.2 & 9.0 \\
\hline $2--$ & 369 & 59.8 & 40.2 & 14.8 & 557 & 22.3 & 221 & 8.8 & 21.5 & 44.4 & 22.6 & 34.1 & 13.5 \\
\hline $3 \ldots$ & 425 & 65.1 & 34.9 & 16.9 & 762 & 30.3 & 277 & 11.0 & 18.9 & 40.1 & 22.8 & 41.0 & 14.9 \\
\hline 4 & 348 & 59.3 & 40.7 & 12.6 & 797 & 28.9 & 206 & 7.5 & 17.7 & 40.3 & 18.3 & 42.0 & 10.9 \\
\hline 5 & 385 & 63.7 & 36.3 & 14.0 & 835 & 30.4 & 245 & 8.9 & 17.8 & 38.9 & 20.0 & 43.3 & 12.7 \\
\hline 6 & 415 & 62.0 & 38.0 & 10.1 & 1,147 & 28.0 & 257 & 6.3 & 18.0 & 36.3 & 16.5 & 45.7 & 10.3 \\
\hline $7 \ldots$ & 404 & 58.8 & 41.2 & 16.3 & 795 & 32.0 & 238 & 9.6 & 17.6 & 41.8 & 20.6 & 40.6 & 12.1 \\
\hline $8 \ldots$ & 280 & 61.4 & 38.6 & 19.7 & 290 & 20.4 & 172 & 12.1 & 23.5 & 47.4 & 28.1 & 29.1 & 17.2 \\
\hline 9 & 225 & 63.5 & 36.5 & 8.4 & 730 & 27.3 & 143 & 5.3 & 20.4 & 36.0 & 13.4 & 43.6 & 8.5 \\
\hline 10 & 461 & 67.8 & 32.2 & 12.7 & 1,075 & 29.7 & 313 & 8.6 & 17.9 & 38.2 & 18.8 & 43.9 & 10.6 \\
\hline Average & 370 & 61.9 & 38.1 & 13.5 & 809 & 27.6 & 230 & 8.4 & 19.5 & 39.8 & 19.7 & 40.7 & 12.0 \\
\hline
\end{tabular}

In selecting nuts for the two preceding series of analyses no attempt was made to secure uniformity as to size and age. On the contrary, they were picked out with a view of obtaining fairly representative samples of the largest and of the smallest, as well as of the most and of the least mature in each pile, so that they would vary through a wide range of color and weight. On comparing the two lots it will be seen that the results agree very closely. Series XI averages a little higher in the total yield of copra, but the oil content of this copra is somewhat lower than in Series $\mathrm{X}$, so that they yield almost exactly the same quantity of oil per nut. The proportion of husk taken from the seashore nuts (41.8) is much larger than it is from those gathered from the interior (32), but 
this is compensated for by the fact that the percentage of milk in the nut, free from husk, and of water in the fresh meat is considerably lower in the former than in the latter. Therefore it appears to be very evident that the superiority of trees growing near the sea is solely due to the quantity and not to the quality of nuts they produce.

Analyses of large numbers of nuts.-As a check on these last results, secured on a small scale, it was decided to determine the actual weight of the various products of the coconut under the conditions ordinarily obtaining in the manufacture of commercial copra, and, with this end in view, 1,000 nuts were procured from trees growing near the seashore and the same number from those standing in the interior. $\Lambda$ fter lying for one month the nuts were put through the regular process for making copra which has previously been described. The weight in pounds of the whole nuts, husks, meat and shells, dried shells, and copra was determined directly on an ordinary Fairbanks scale, the meat and milk being obtained by difference. Five hundred nuts from each lot were sun dried and 500 grill dried and the resulting weight of copra multiplied by two to give the yield of 1,000 nuts by each method. For the determination of moisture and oil in this copra, twenty samples were taken from each lot, cut into small pieces, and quartered down to about 100 grams. 'The moisture was determined at once, after which the copra was sealed and sent to Manila to secure the determination of the oil content. Both moisture and oil were determined in triplicate.

Serifs XII.

\begin{tabular}{|c|c|c|c|c|c|c|c|c|c|}
\hline \multirow{2}{*}{\multicolumn{4}{|c|}{ l'ortion determined. }} & \multicolumn{4}{|c|}{ Seashore nuts. } & \multicolumn{2}{|c|}{ Inland nuts. } \\
\hline & & & & \multicolumn{2}{|c|}{$\begin{array}{l}\text { Weight in } \\
\text { kilos. }\end{array}$} & \multicolumn{2}{|c|}{ Per cent. } & $\begin{array}{c}\text { Weight in } \\
\text { kilos. }\end{array}$ & Per cent. \\
\hline \multicolumn{4}{|l|}{ Weight of $1,000 \ldots$} & \multirow{4}{*}{\multicolumn{2}{|c|}{$\begin{array}{r}2,363 \\
897 \\
1,466 \\
929\end{array}$}} & \multirow{3}{*}{\multicolumn{2}{|c|}{$\begin{array}{r}100.0 \\
38.0 \\
62.0\end{array}$}} & \multirow{3}{*}{$\begin{array}{r}2,286 \\
703 \\
1,582\end{array}$} & 100.0 \\
\hline \multirow{2}{*}{\multicolumn{4}{|c|}{$\begin{array}{l}\text { Husks } \\
\text { Nuts minus husks }\end{array}$}} & & & & & & 30.8 \\
\hline & & & & & & & & & 69.2 \\
\hline \multicolumn{4}{|c|}{ Neat and shell } & & & & & 979 & 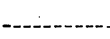 \\
\hline \multirow{2}{*}{\multicolumn{4}{|c|}{$\begin{array}{l}\text { Milk } \\
\text { shell (dry) - }\end{array}$}} & \multicolumn{2}{|c|}{537} & \multicolumn{2}{|c|}{22.7} & 603 & 26.4 \\
\hline & & & & \multirow{2}{*}{\multicolumn{2}{|c|}{$\begin{array}{l}282 \\
647\end{array}$}} & \multirow{2}{*}{\multicolumn{2}{|c|}{$\begin{array}{l}11.9 \\
27.4\end{array}$}} & 291 & 12.7 \\
\hline Meat - & & & & & & & & 688 & 30.1 \\
\hline \multirow{3}{*}{ Portion determined. } & \multicolumn{4}{|c|}{ Seashore nuts. } & \multicolumn{5}{|c|}{ Inland nuts. } \\
\hline & \multicolumn{2}{|c|}{ Sun dried. } & \multicolumn{2}{|c|}{ Grill dried. } & \multicolumn{3}{|c|}{ Sun dried. } & \multicolumn{2}{|c|}{ Grill dried. } \\
\hline & $\begin{array}{l}\text { Weight } \\
\text { in kilos. }\end{array}$ & $\begin{array}{l}\text { Per } \\
\text { eent. }\end{array}$ & $\begin{array}{l}\text { Weight } \\
\text { in kilos. }\end{array}$ & $\begin{array}{l}\text { Per } \\
\text { cent. }\end{array}$ & We & $\begin{array}{l}\text { eight } \\
\text { kilos. }\end{array}$ & $\begin{array}{l}\text { Per } \\
\text { cent. }\end{array}$ & $\begin{array}{l}\text { Weigh } \\
\text { in kilo }\end{array}$ & $\begin{array}{c}\text { Per } \\
\text { cent. }\end{array}$ \\
\hline Copra_____ & 302.1 & 12.8 & 330.2 & 14.0 & & 322.9 & 14.1 & 333. & 14.6 \\
\hline Oil_- & 182.2 & 7.7 & 198.9 & 8.4 & & 191.1 & 8.4 & 189. & 8.3 \\
\hline Moisture in copra & - n- & 9.2 & ----- & 8.6 & & & 9.8 & & 10.1 \\
\hline Oil in copra & --o---- & 60.3 & - & 60.2 & - & & 59.2 & -........ & 57.0 \\
\hline
\end{tabular}


This work, performed as it was on a large scale, agrees rather more closely with the results obtained from the series of ten nuts each than was to be expected. Here, again, it may be observed that the proportion of husk from the seashore nuts is considerably higher than it is from those from the interior, while the total amount of water is correspondingly less, so that nuts from the two localities yield practically the same amount of copra and oil.

While weighing out 1,000 nuts from the seashore trees it was found that 55 of them, or 5.5 per cent, were in such a bad condition as to be unfit for making copra, and fresh nuts had to be substituted. Out of the same number from the interior only 15 were spoiled. The cause of this difference is probably found in the fact that the nuts from trees near the sea fall upon harder ground and are therefore more apt to become bruised and injured, and it is very possible that the inferior yield of sun-dried as compared with kiln-dried copra, in the case of the seashore nuts, is due to this. Given perfectly sound coconuts, the two methods of drying should produce equal amounts of copra, but a green nut, or one which has begun to decay, would undoubtedly be more subject to the attacks of mold, bacteria, and insects during the comparatively long alternate heating and cooling incident to the sun-drying process than if it were dried quickly at a higher temperature.

The figures obtained in this last series on a commercial basis establish, even more firmly than do the results of analyses alone, the fact that there is practically no difference in quality between the nuts gathered along the seashore and those from farther inland. They should also be of some value as representing the average yield in copra and oil from nuts produced in the southern parts of the Islands.

\section{NUTS FROM DAVAO.}

The following analyses were made of ripe coconuts, eollected near Davao, about 1 mile inland from the sea. In this region two varieties of trees have been noticed, one producing large nuts rather pointed in shape, the other bearing a smaller, rounder fruit.

Series XIII consists of ten of the small nuts, Series XIV of the large variety. On examining these figures it will be noticed that Series XIII shows very much the same proportion of its various constituents, as well as the total of oil, as the average lot of ripe nuts from San Ramon.

Series XIV excels in total weight of oil simply because it is made up of larger nuts. 'The percentage of oil in the nut, free from husk, is the same in both series. The nuts in these two series were fairly uniform in composition, with the exception of No. 7 in Series XIV, which had a total weight of only 92 grams of oil, less than one-half of the average amount. 
Serifos XIII.-Maran nuts, small.

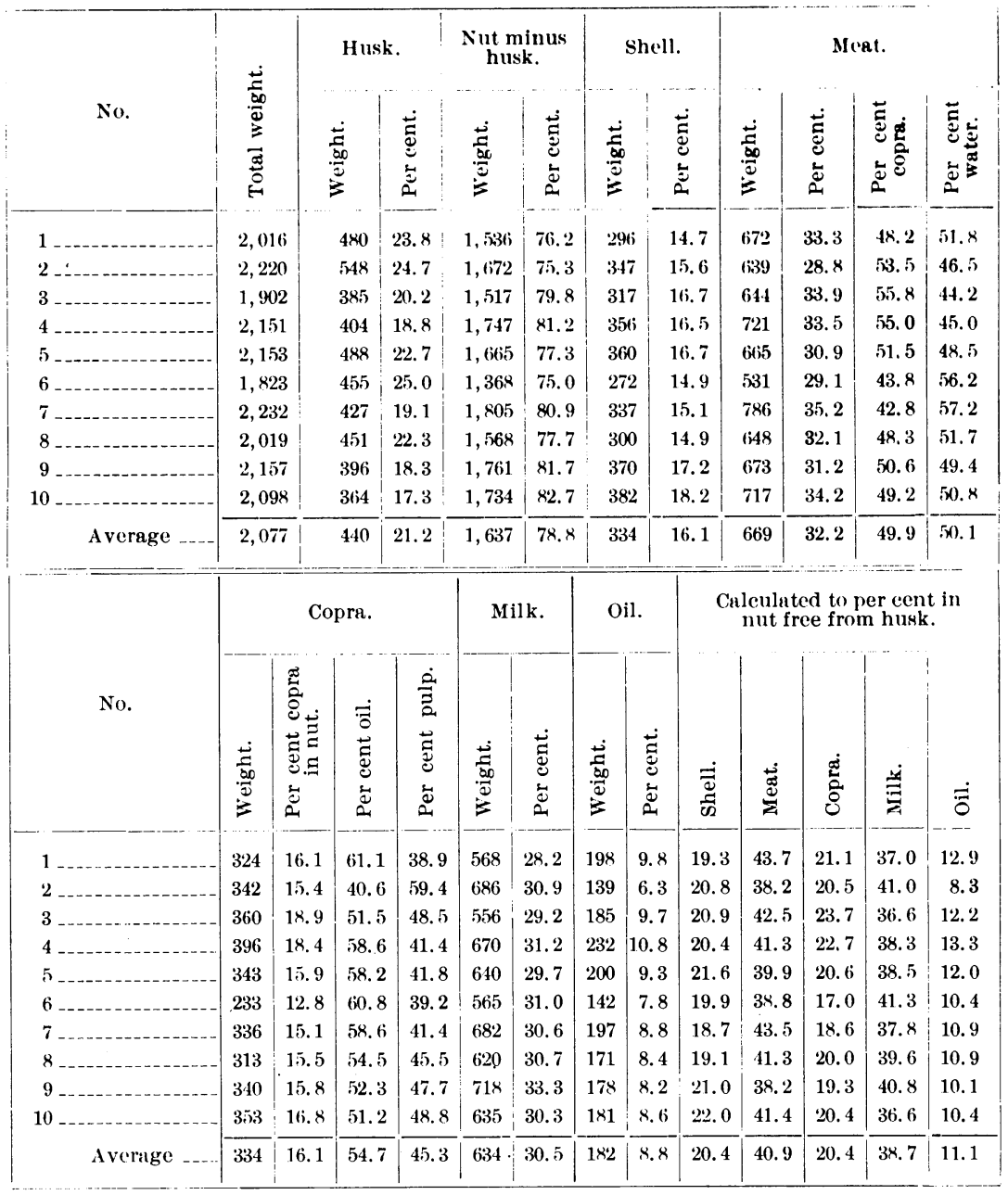




\section{2}

Series XIV.-Davao nuts, large.

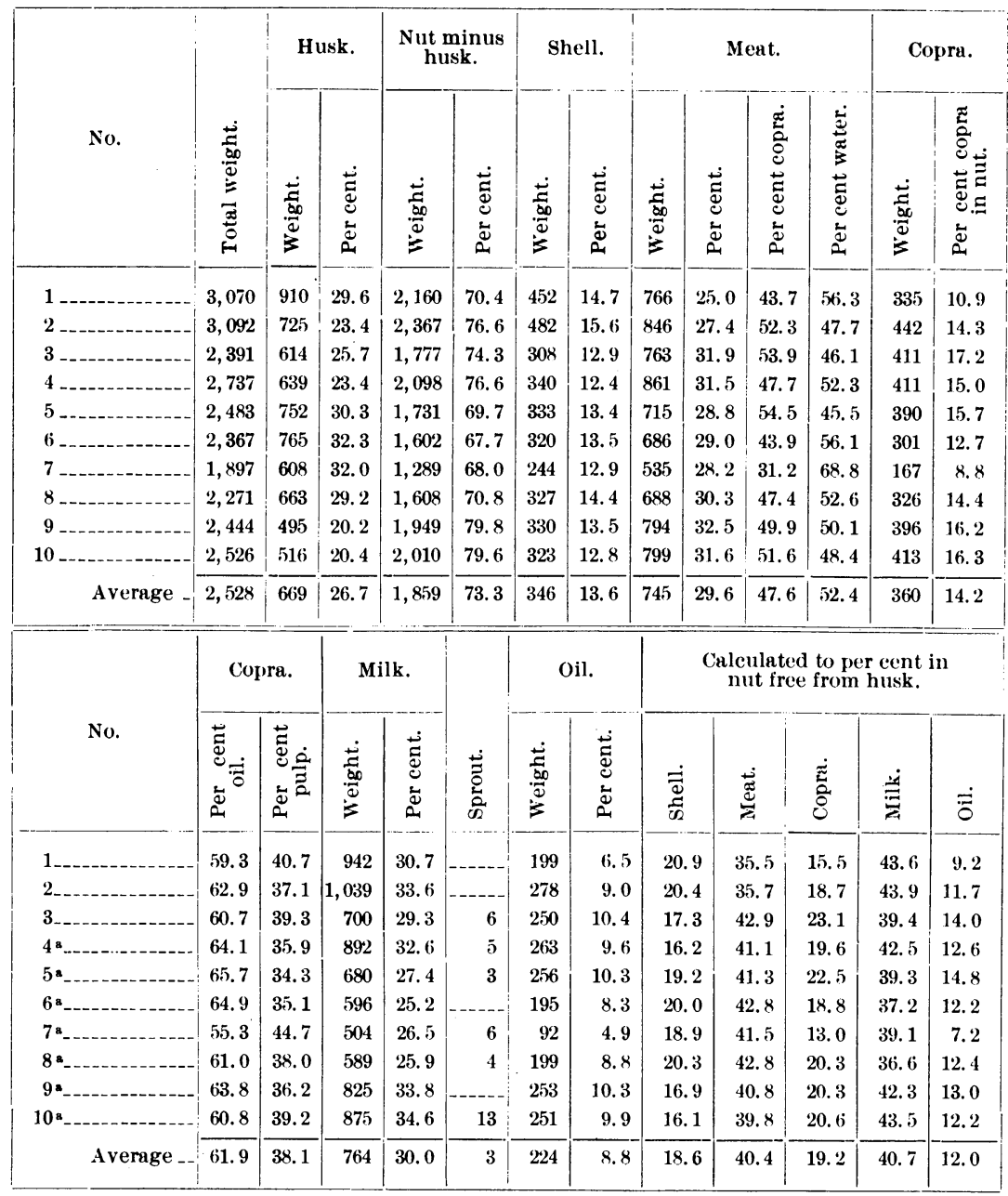

- Oil separated from the milk, hence the nuts were very ripe. 


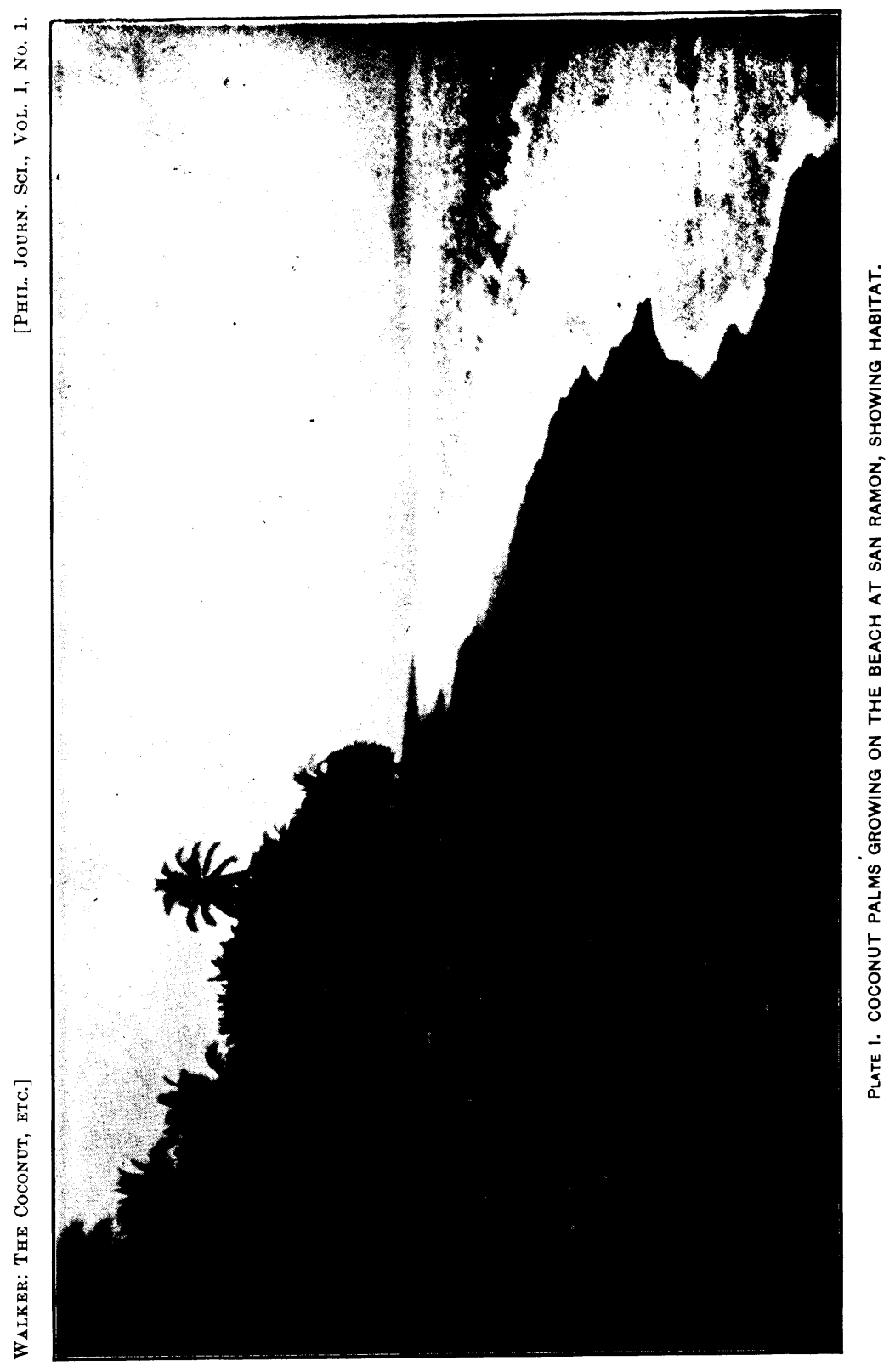




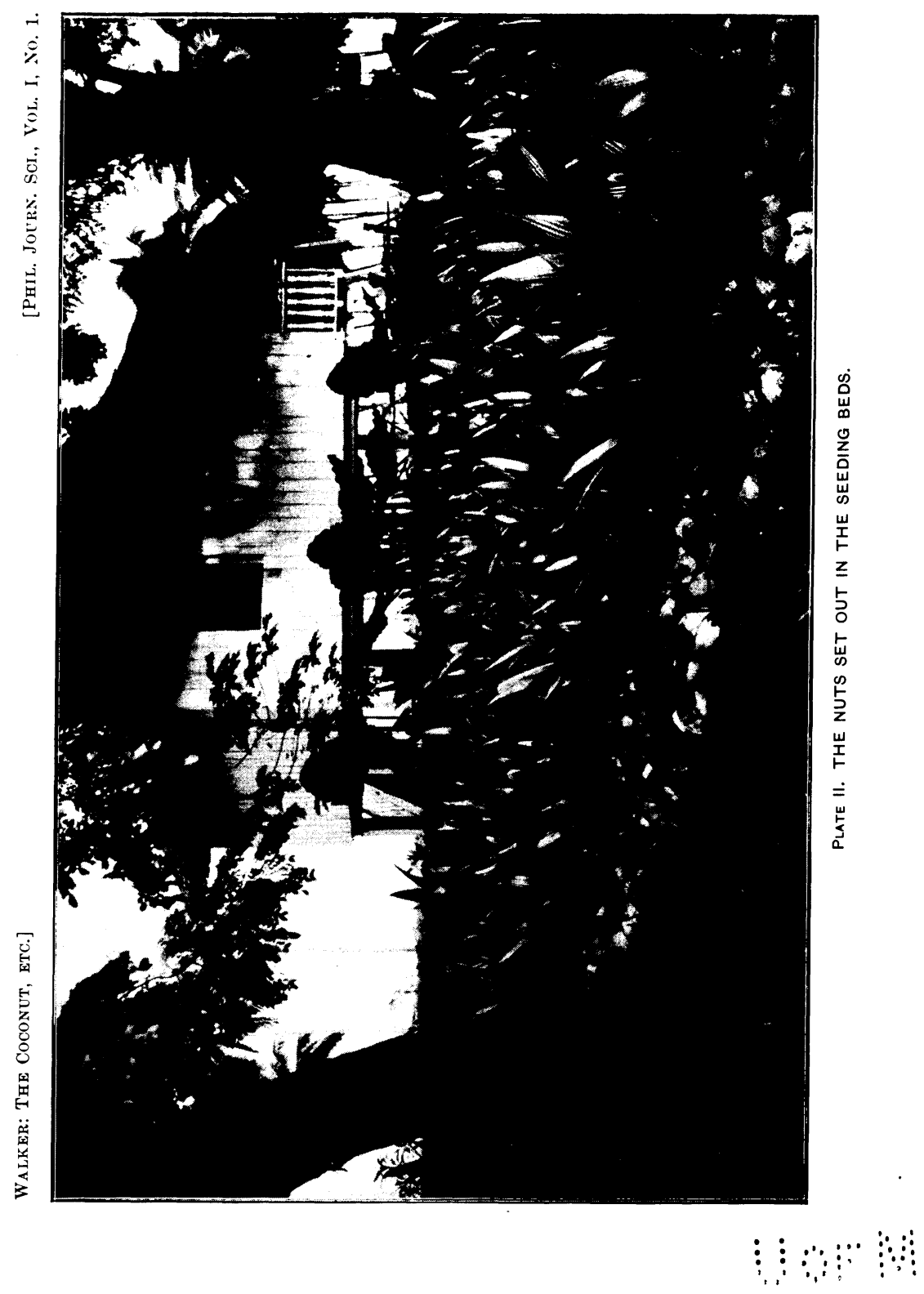





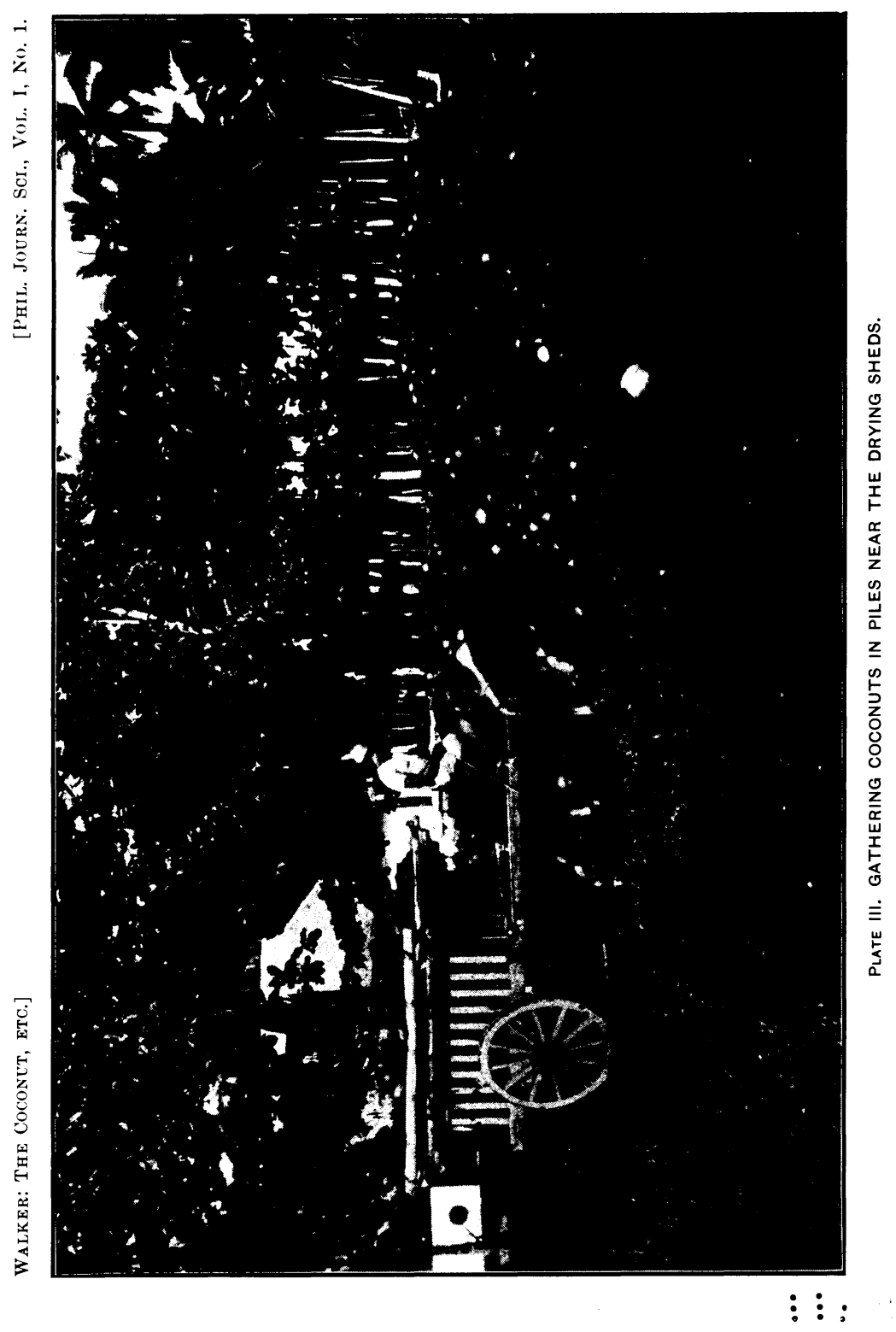


: 


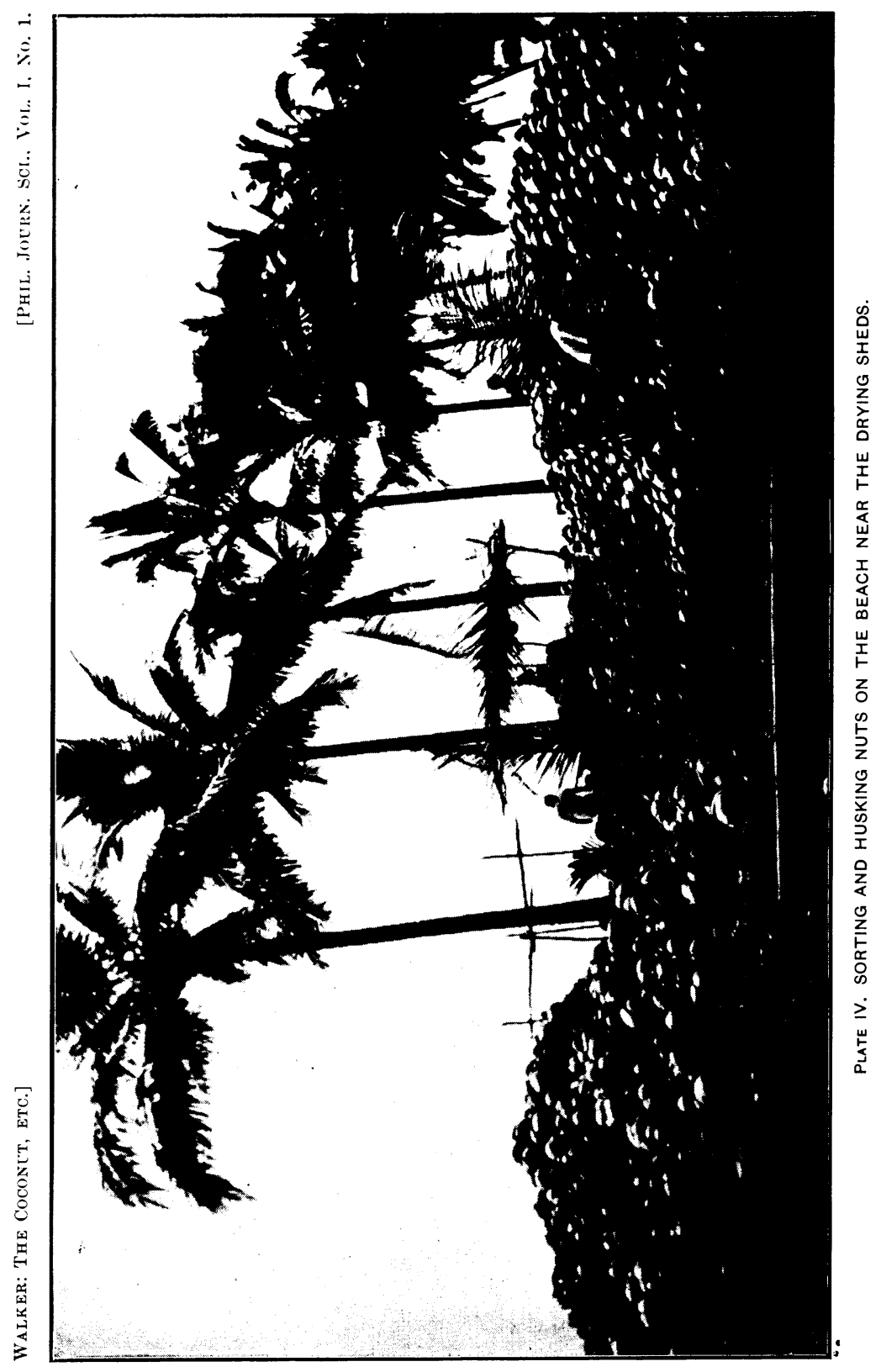


: $: \vdots \vdots$ 


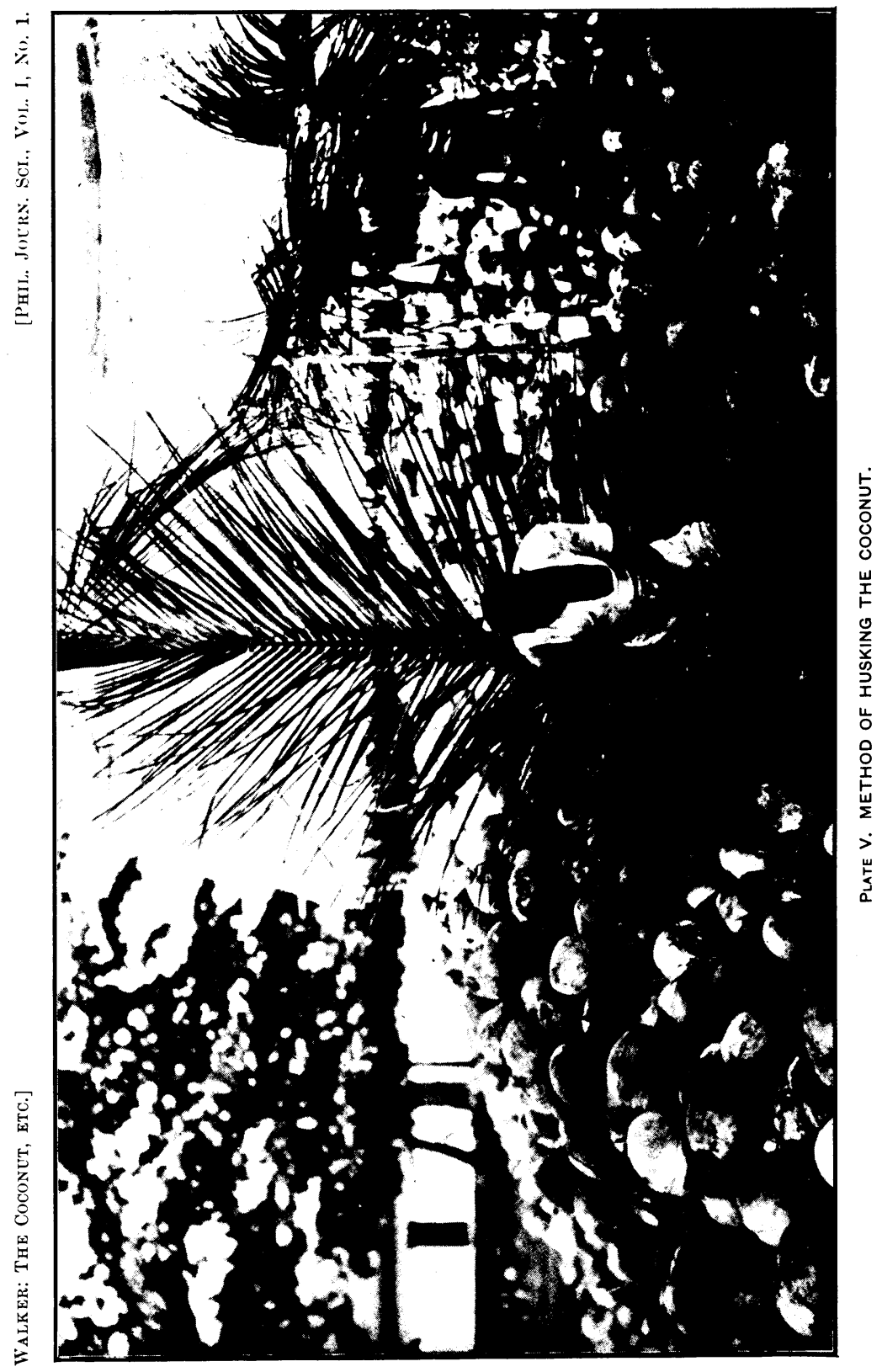

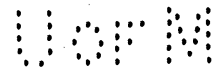





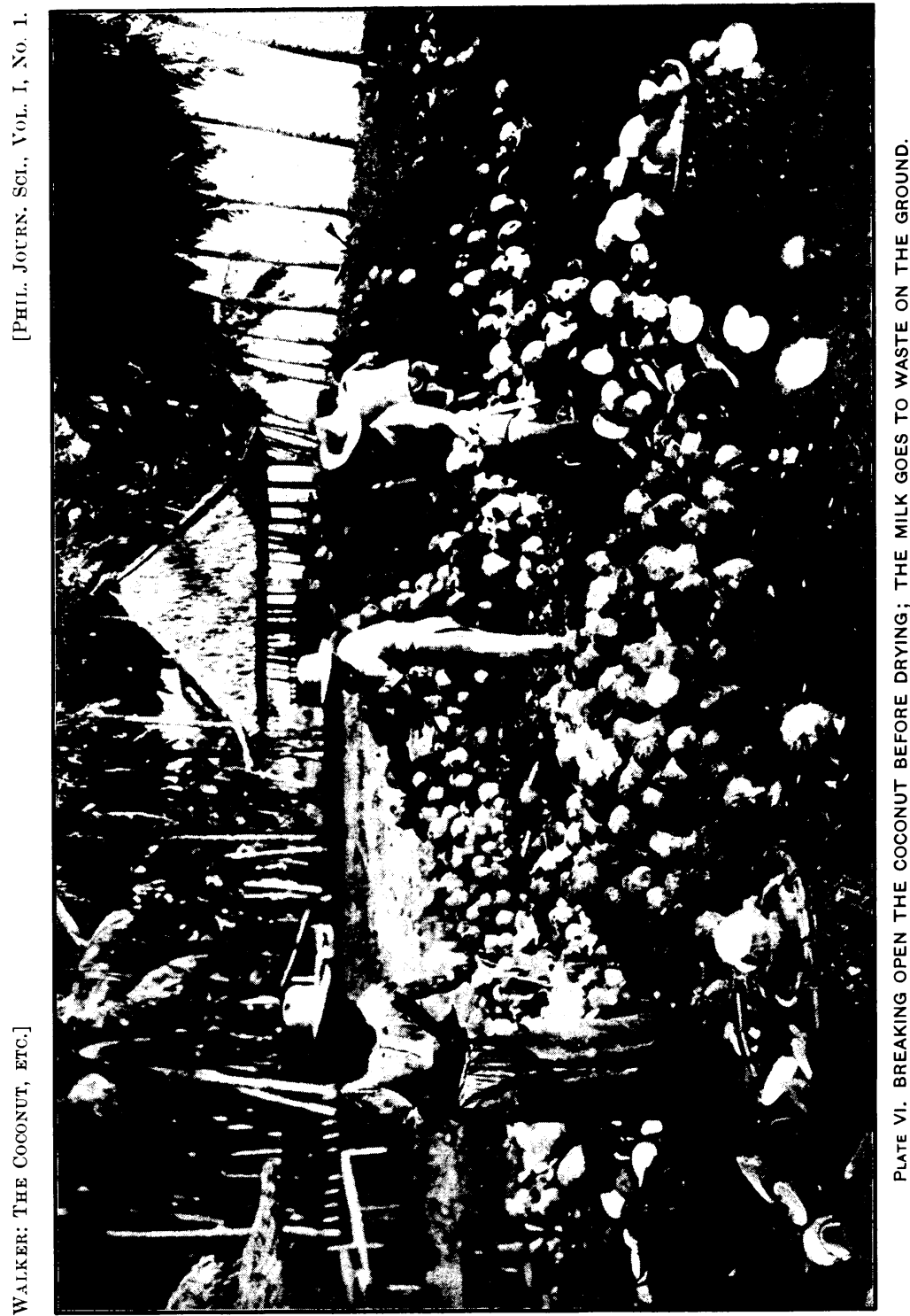


$\therefore \cdots$ 


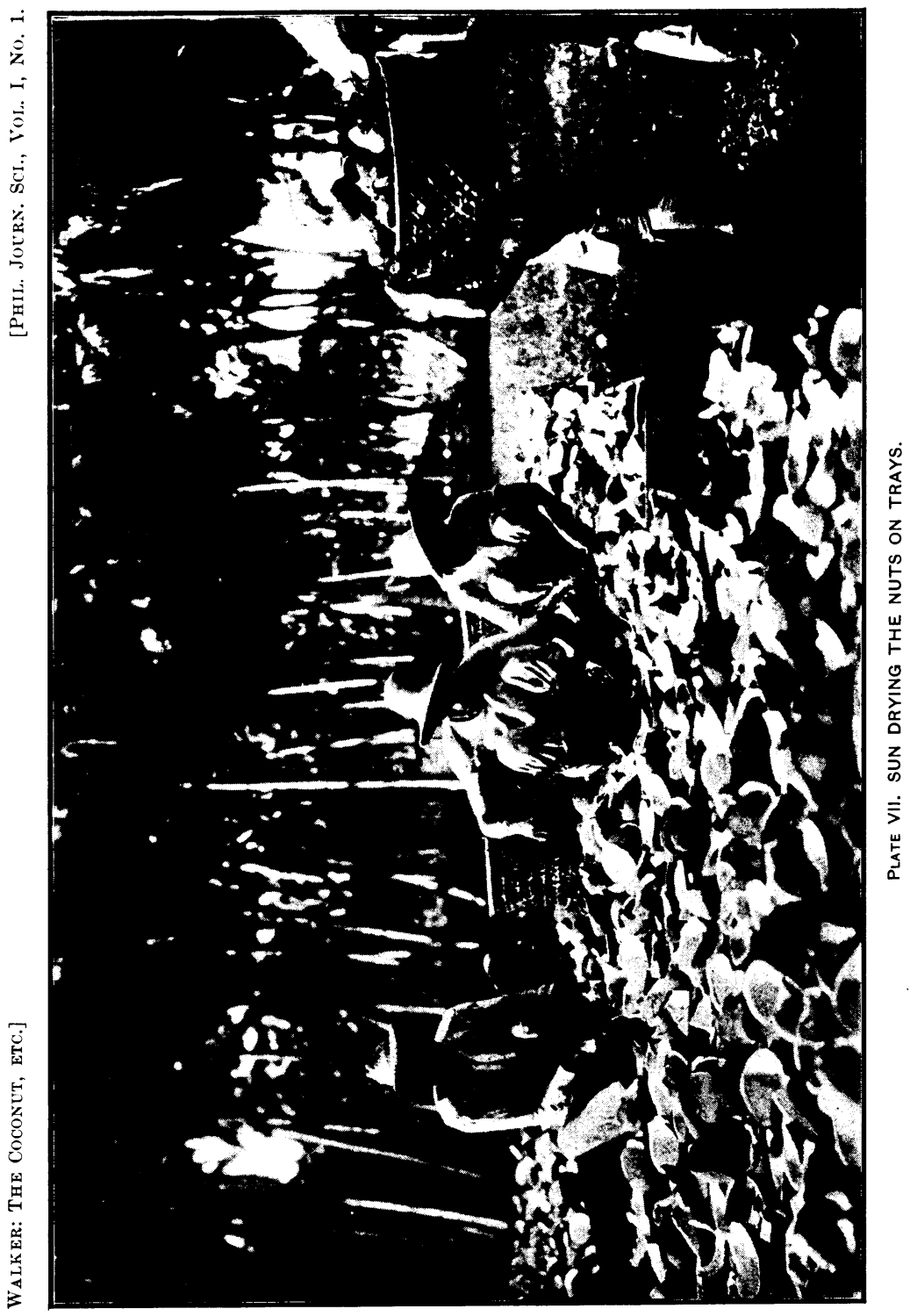




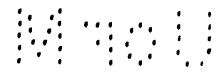




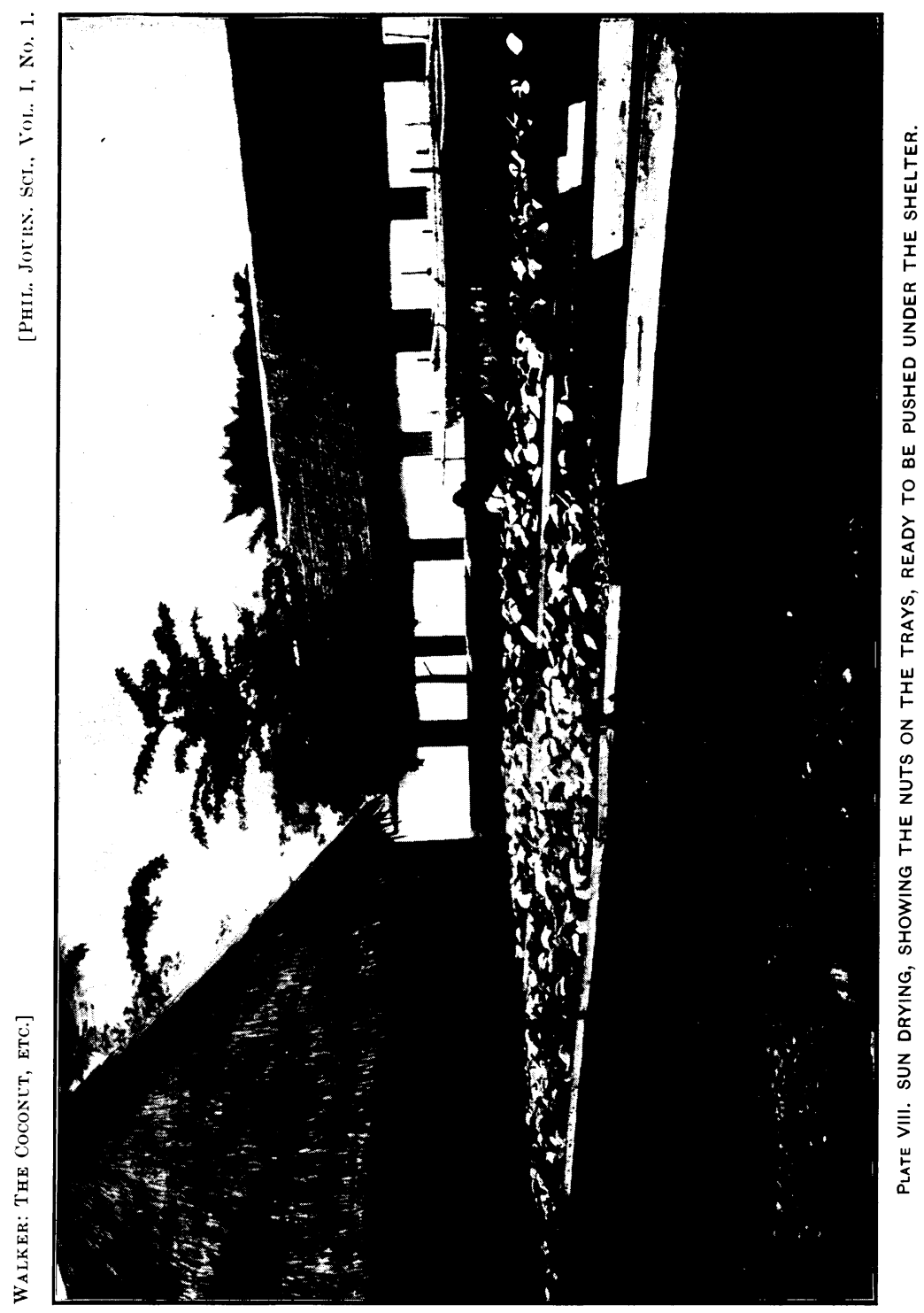


: 


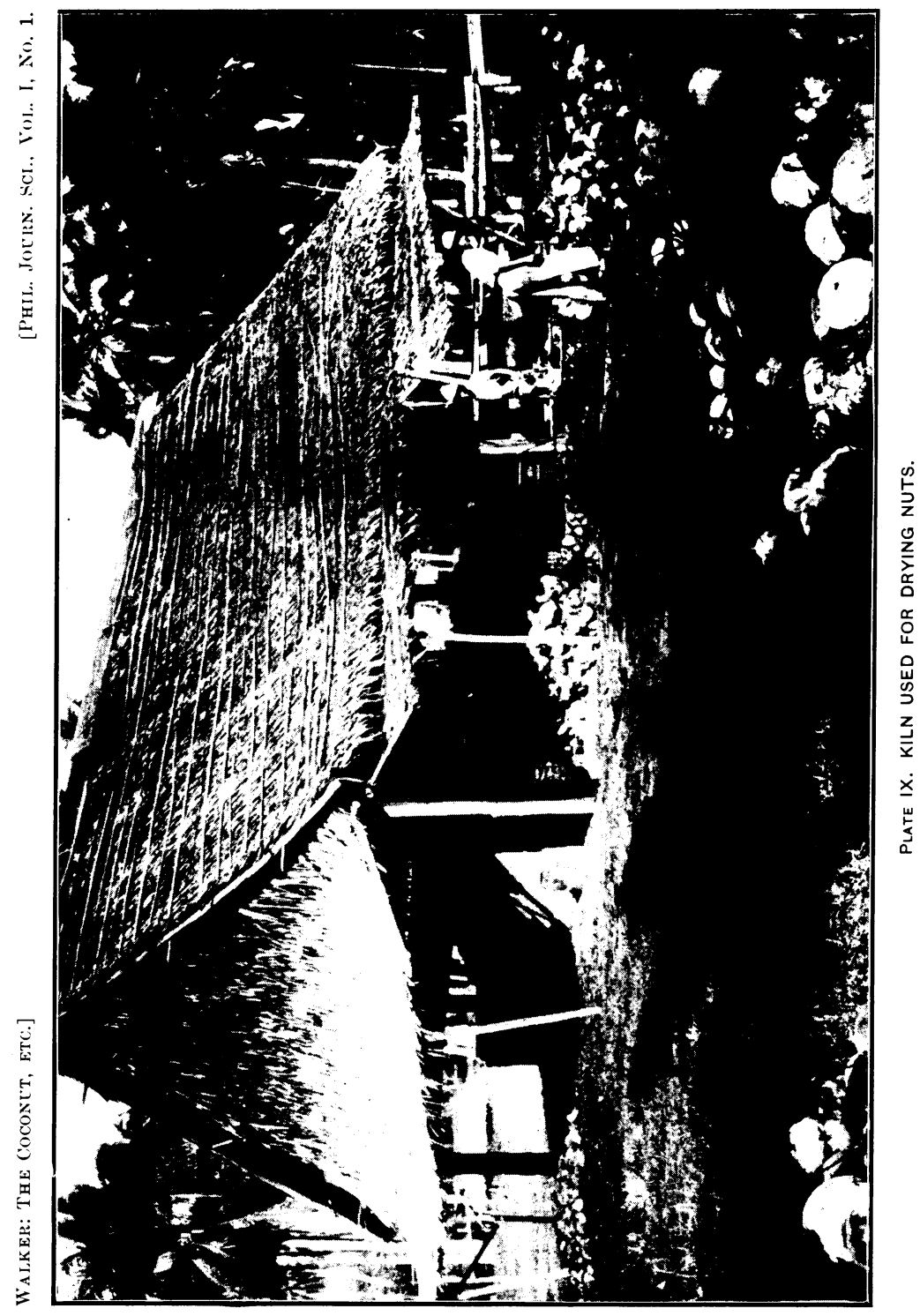

药 



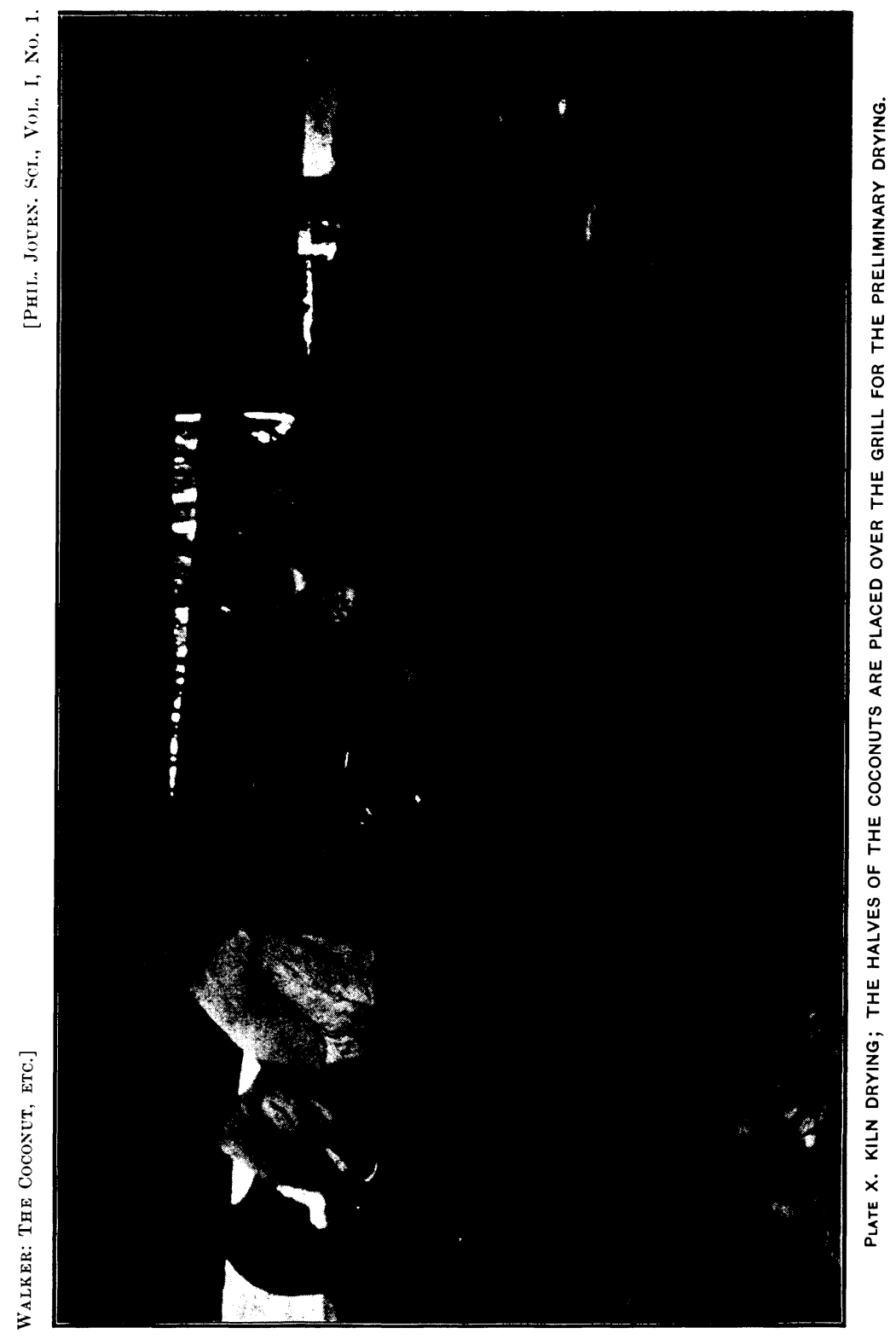




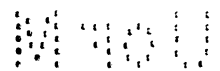




\title{
THE KEEPING QUALITIES AND THE CAUSES OF RANCIDITY IN COCONUT OIL.
}

\author{
By Herbert S. WALKer. \\ (From the Chemical Laboratory, Bureau of Science.)
}

In almost every work on fats and oils, coconut oil is cited as being especially prone to become rancid. Lewkowitsch ${ }^{1}$ states that, when fresh, it possesses a bland, pleasant taste and odor, but that on standing it quickly becomes rancid. Samples analyzed by him contained from 5 to 25 per cent of free acid. Schestakoff ${ }^{2}$ says that pure coconut oil shows an acid value (milligrams of caustic potash) of from 2 to 5 . On standing under abnormal conditions, this may in one year rise to 60 or 70.

Coconut oil is in enormous demand as the basis of edible products such as "vegetable butter," etc., and therefore it is of the utmost importance to be able to produce an oil which, as nearly as possible, is free from fatty acids, rancid odor or taste, and which at the same time may be shipped without fear of deterioration.

The experiments to be described were undertaken with the view of discovering the conditions which induce a rapid deterioration of coconut oil, and, if possible, of ascertaining a means of improving its keeping qualities. In the course of this work it was noticed that the oil does not change with as great rapidity as is generally believed to be the case. The ordinary commercial oil, bought in Manila, contains from 5 to 10

${ }^{1}$ Lewkowitsch: Chemical Analysis of Oils, Fats, and Waxes.

${ }^{2}$ Schestakoff : über den Gehalt an freien Fettsäuren natürlicher Fette und öle. Chem. Rev. Fett. u. Harz. Ind. 9, 180. 
per cent free acid, and there is no very great increase in acidity even on prolonged standing. Mr. Richmond, of this laboratory, in some experiments on a commercial oil, showed that it was not affected by passing a current either of dry or of moist air through the liquid for five days. An oil prepared by drying fresh coconut meat at $80^{\circ}$ to $90^{\circ}$ C., and then extracting with petroleum ether, was sterilized and sealed in a glass tube on August 16, 1904. At that time it contained 0.13 per cent free acid (calculated as oleïc). This sample was allowed to stand until February 21, 1905, and it was then again tested, with the result that it was in as good condition as at the time of the previous examination; there had been absolutely no increase in free acid. A sample of the same oil, kept in a sealed tin tube, on February 21, 1905, contained 0.34 per cent of free acid, an increase of only 0.21 per cent in six months. This same oil was allowed to remain in tin, unsealed, until April 11, 1905; the acidity had then increased to 0.46 per cent. On further standing in a glass-stoppered bottle until August 16, 1905, the figure was 0.50 per cent. An expressed oil from the same preparation of copra, kept under similar conditions, showed:

$\begin{array}{ll} & \text { Per cent. } \\ \text { February } 21 & 0.77 \\ \text { April } 11 & \\ \text { August } 16 & \end{array}$

This oil, from the start, had a slightly burnt odor and taste and in time it deposited a dark-brown sediment. However, neither of the two oils showed any signs of "rancidity" and even after a year had elapsed they were almost as pleasant to the taste as when first prepared.

In order more fully to study the effect of age and method of preparation on the keeping qualities of coconut oil, the following samples were prepared, their condition noted, and their exact titer determined. These oils will be allowed to stand for several years if necessary, until final results are obtained as to their respective rates of deterioration, but in the meanwhile the change up to the present time is given in the table which follows:

DESCRIPTION OF OILS USED AND DESCRIBED IN TABLE I.

(A) Expressed oil from vacuum-dried copra. Has been heated for two hours at $100^{\circ}$ and filtered twice through paper. A light-colored, clear oil with the characteristic coconut taste and odor.

(B) An oil similar in every respect to "A" except that it was prepared from copra dried at $80^{\circ}$ to $90^{\circ}$, without vacuum.

(1) Fresh coconut meat grated and dried at $80^{\circ}$ to $90^{\circ}$ on August 16, 1904 ; was allowed to stand in a covered specimen jar until March 11, 1905. At that time it was still of a pleasant odor and taste, although both odor and taste were rot quite as good as when the specimen was freshly prepared. No mold growth was presert. A sample of oil was expressed from a portion of this copra by using a hydraulic press with a final pressure of 450 kilograms per square cen- 
timeter. This oil, after filtration, was of a light-yellow color and it was of a pleasant, although slightly burnt, odor and taste.

(2) Oil No. 1 was heated at $100^{\circ}$ for three hours, while at the same time a current of air in a partial vacuum was passed through it. This process leaves the color and free acid unchanged but removes almost all of the burnt odor, leaving a bland, almost tasteless, oil.

(3) An oil from the same copra as Nos. 1 and 2 but prepared by extraction with petroleum ether. Afterwards it was treated in the same manner as No. 2. 1t differs from Nos. 1 and 2 in being practically colorless.

(4) Commercial coconut oil treated with alcohol and animal charcoal and then filtered; the alcohol was afterwards distilled and recovered. This oil was rather unpleasant to the taste, but it had no odor.

(5) Commercial coconut oil treated with live steam; this removes the odor, but the unpleasant taste remains.

(6) Fresh meat, ground and dried in vacuum at $70^{\circ}$ to $80^{\circ}$. The oil was expressed and once filtered; it possessed a very pleasant coconut-like odor and taste. lt still contained a considerable amount of sediment.

(7) Coconuts cut in halves and dried in vacuum at $75^{\circ}$ to $85^{\circ}$. The oil expressed and filtered twice. It had a very pleasant odor and taste.

(8) The same oil as $\mathrm{No.}^{\bullet} 7$, heated at $100^{\circ}$ for one and one-half hours and filtered hot.

(9) The same as No. 7 , heated at $100^{\circ}$ for one and one-half hours, while at the same time a current of air was passed through the oil under partial vacuum. Filtered hot and bottled.

(10) Fresh coconut meat, ground and pressed in a hand press to remove most of the milk. Afterwards this meat was dried completely by spreading it in the sun for about five hours. The oil expressed from this copra was almost water white and without taste or odor.

(11) Coconuts split in halves and dried in the sun for five days. Ground and expressed. Yielded a cloudy, light-colored oil, very hard to filter, with a peculiar but not unpleasant taste and odor. This sample was strained through cloth but not filtered.

(12) Same as No. 11, strained and filtered slowly through paper.

(13) Same as No. 11, heated at $100^{\circ}$ for two hours and filtered through paper.

(14) Fresh nuts, split in halves and allowed to stand during one week in the air at room temperature (about $30^{\circ}$ ). A vigorous mold growth and an unpleasant odor developed. This moldy meat was dried in a vacuum and the oil was expressed. This was highly colored and was rather unpleasant to taste and smell.

(15) Commercial coconut oil shaken with 2 per cent of solid calcium oxide (burned lime), heated to $100^{\circ}$ and filtered. The filtrate was treated with animal charcoal and again filtered; there resulted a colorless oil which was very free from an unpleasant odor or taste.

(16) The same copra as that used for No. 1; was allowed to stand one month longer in an open jar, then expressed.

(17) Oil expressed from vacuum-dried copra which had stood for one month exposed to the air; the oil was heated to $100^{\circ}$ and filtered.

(18) Expressed from sun-dried copra and treated in the same manner as No. 17. Both of these samples were of as pleasant a taste as oils from fresh copra.

(19) Vacuum-dried copra which had stood in a closed desiccator ơvec vatier fớr, one month, and which had accumulated a very decided growth of nicld.' "It wis' dried for one hour and expressed. The oil had a considerable color and was slightly unpleasant as to taste and odor. Heated to $100^{\circ}$ and filtered. 
(20) Sun-dried copra treated in the same way as No. 19. Yielded an oil somewhat darker in color but otherwise much the same as No. 19. Filtered without heat.

(21) Same as No. 20 , heated to $100^{\circ}$ before filtering.

(22) The same copra as that used for samples 1 and 16 was allowed to stand for three weeks over water and for one week in air, and then dried and pressed. A vigorous mold growth appeared in the copra and a peculiar ethereal odor was apparent. The oil itself was of a light-yellow color, with a pungent, rather unpleasant, odor and an extremely disagreeable taste.

(23) Expressed from commercial copra, first quality, sun dried, Tacloban, Leyte. The unfiltered oil is dark colored and cloudy, depositing a black sediment.

(24) Same as No. 23, filtered. Almost colorless.

(25) Expressed from commercial copra, grill dried, Laguna (second quality). Not filtered.

(26) Same as No. 25, filtered. Light yellow in color.

(27) Expressed from commercial copra, grill dried, Romblon (considered second quality). The filtered oil is light yellow in color.

(28) Expressed from commercial copra, first quality, sun dried, Iloilo. The filtered oil is light yellow in color. ${ }^{3}$

(29) "Langis" coconut oil, prepared by the customary native process of grating the fresh meat, exhausting it repeatedly with water, and boiling down the emulsion thus obtained until it is nearly dry. The oil is then poured off from the brown coagulum which sinks to the bottom of the vessel. A freshly prepared oil, isolated in this manner, is very light in color and it possesses a decidedly pleasant coconut odor and taste. Before filtration it is more or less turbid, owing to the presence of a small amount of water and of albuminoids.

(30) Same as No. 29, filtered. The oil is water white.

(31) Best grade commercial coconut oil, probably made from fresh meat. It is light colored but very turbid and contains considerable water and suspended matter.

(32) Commercial coconut oil, probably made from copra. Very clear but highly colored.

(33) Commercial coconut oil, Manila. Probably made from fresh meat. It contained considerable suspended matter and water.

(34) Commercial coconut oil, Cebu. A highly colored "rancid" oil. Considerable sediment in the bottom of the bottle.

(35) Commercial coconut oil, Tayabas. A highly colored rancid oil made from copra. It is only a few months old.

The following table shows the change in the amount of free acid which has been produced in these oils while they were standing from the time of their expression up to the date of writing. The free acid was determined in each case by dissolving a known weight (about 5 grams) of oil in 50 cubic centimeters of neutralized absolute alcohol, and

${ }^{3}$ The copras from which these last six samples of oils 'were made were secured through the courtesy of Messrs. Smith, Bell \& Co. and the Compañia General de Tabacos de Filipinas, and taken directly from their warehouses. The samples obtained were the ordinary grades of copra, ready for export, and had been stored for about two months, during the dry season. The oils, while not especially

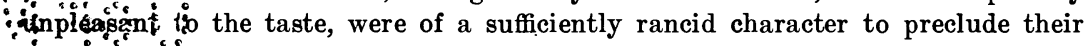
use as ediblé products unless they were first subjected to a refining process. 
then titrating with aqueous $\frac{\mathrm{N}}{10}$ potassium hydroxide, using phenolphthaleïn as an indicator :

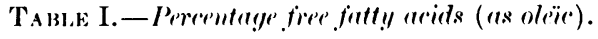

\begin{tabular}{|c|c|c|c|c|}
\hline No. & At start. & $\begin{array}{c}\text { Two } \\
\text { months. }\end{array}$ & $\begin{array}{l}\text { Four } \\
\text { months. }\end{array}$ & $\underset{\text { months. }}{\operatorname{six}}$ \\
\hline$A^{\mathrm{a}}$ & 0.06 & 0.06 & 0.09 & 0.60 \\
\hline B .- & 0.06 & 0.06 & 0.08 & b 0.48 \\
\hline $1 .-$ & 1.2 & 1.3 & 1.5 & 1.9 \\
\hline 2 & 1.2 & 1.5 & 1.5 & 1.7 \\
\hline 3 & 1.4 & 1.6 & 2.1 & 2.6 \\
\hline 4 & 5.3 & --------- & $\overline{5} .9$ & 6.1 \\
\hline 5 & 5.5 & ----- & ----- & 7.6 \\
\hline - & 0.10 & 0.16 & 0.19 & 0.30 \\
\hline 7 & 0.16 & 0.18 & 0.19 & 0.27 \\
\hline 8 & 0.16 & 0.14 & 0.19 & 0.30 \\
\hline $9_{-\ldots-n}$ & 0.16 & 0.16 & 0.18 & 0.25 \\
\hline $10 \ldots$ & 0.16 & 0.16 & 0.21 & 0.28 \\
\hline 11 & 0.13 & 0.18 & 0.25 & 0.28 \\
\hline 12 & 0.13 & 0.10 & 0.10 & 0.14 \\
\hline 13 & 0.13 & 0.09 & 0.09 & 0.15 \\
\hline 14 & 3.5 & 3.7 & 4. 0 & 4.3 \\
\hline 15 & 0.32 & ---- & 0.88 & --- \\
\hline 16 & 1.6 & 1.7 & 2.0 & ---- \\
\hline 17 & 0.09 & 0.09 & 0.14 & 0.16 \\
\hline 18 & 0.16 & 0.18 & 0.25 & 0.27 \\
\hline 19 & 1.18 & 1.14 & 1.34 & 1.58 \\
\hline 20 & 0.69 & 0.69 & 0.74 & 0.85 \\
\hline $21 \ldots$ & 0.69 & 0.69 & 0.74 & 0.82 \\
\hline $22_{-}$ & 23.3 & - & ----- & $-\cdots$ \\
\hline 23 & 1.4 & 1.6 & 1.8 & 2.0 \\
\hline $24_{---}$ & 1.4 & 1.5 & 1.7 & 1.8 \\
\hline $25 \ldots$ & 2.6 & 3.4 & 3.6 & 3.9 \\
\hline 26 & 2.6 & 2. 6 & 3.1 & 3.5 \\
\hline $27 \ldots$ & 2.1 & 2.4 & 2.5 & 2.8 \\
\hline 28 & 3.0 & 3.5 & 4.0 & 4.7 \\
\hline 29 & 0.08 & 0.38 & 0.60 & 0.69 \\
\hline $30 \ldots$ & 0.08 & 0.13 & 0.16 & 0.19 \\
\hline 31_-_-_- & 2.0 & 2.9 & $\ldots$ & $----n$ \\
\hline 32_- & 6.8 & 7.5 & 7.9 & 8.1 \\
\hline 33_-1 & 5.5 & --_- - & 6.9 & 7.2 \\
\hline 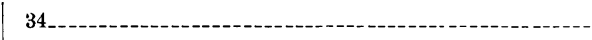 & 8.7 & $---1--1--$ & 10.2 & 11.0 \\
\hline 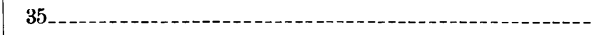 & 5.0 & 5.5 & 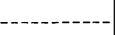 & \\
\hline
\end{tabular}

a Through the courtesy of Prof. A. H. Gill, of the Massachusetts Institute of Technology, I have been given some further data on the keeping qualities of this oil. A sample packed in a sealed tin can was shipped to him at Boston and tested there when it was about three months old. On arrival it was described as being almost water white and of perfectly sweet odor. It then contained only it was described as being almost water white and of perfectly sweet odor. It then contained only same time.

bThis oil was kept in a large bottle. A sample in a small bottle showed an acidity of only 0.09 at this time.

At the present time there has been so little change in any of these samples that no very definite conclusions can be drawn as to the conditions which cause rancidity on standing. However, it may be considered as established that a pure, fresh, coconut oil can be prepared which contains a minimum amount of free acid and which shows no unpleasant 
taste or odor. Such an oil very slowly increases in acidity, and, even after standing for one year under ordinary conditions, may still be edible without further purification. ${ }^{4}$ Commercial oils, on the other hand, which contain from 5 to 10 per cent of free acid when freshly prepared, deteriorate much more rapidly, even though they have been filtered and are free from impurities. For example, No. 32, which, on first examination, had 6.8 per cent of free acid, increased in two months to 7.5 per cent. This oil is very clear, bright, and dry, being entirely free from sediment or turbidity of any kind. The samples prepared by us from fresh copra ranged from 0.06 to 0.16 per cent, the increase in two months being so small as to be almost negligible. Samples Nos. 1, 2, and 3, which contained a little over 1 per cent of free acid when fresh, increased from 0.1 to 0.3 per cent in the same time.

In fact, the increase in free acid to be expected in an oil when it is standing under ordinary conditions may almost be considered as being roughly proportional to its initial acidity. There are also indications that an oil from which albuminoids, etc., have been removed by filtration will retain its original condition better than one containing the above impurities. No. 6, for instance, which has been filtered once but which contained a considerable sediment, increased in four months from 0.10 per cent to 0.19 per cent, while Nos. 7, 8, and 9, oils prepared in a similar manner but filtered more thoroughly, only showed an increase in the same time of from 0.16 per cent to 0.19 per cent of free acid. ${ }^{5}$ This fact was a little more noticeable among oils prepared from sun-dried copra. Samples Nos. 11, 12, and 13 were taken from the same lot of oil, the only difference being that No. 11 was left unfiltered, while the impurities were removed as completely as possible from Nos. 12 and 13 . It will be noticed that No. 11, in six months, shows a total increase of 0.15 per cent free acid, having a little more than double its original acid value. Nos. 12 and 13 have in the same time increased only 0.01 and 0.02 per cent, respectively.

The oils prepared from commercial copra likewise show this distinction to a greater or less extent. No. 25, an unfiltered oil, increased from 2.6 to 3.4 per cent in two months, while No. 26, which is the same oil filtered, shows no change at all. However, contrary to expectations, the difference

"The șudden increase of acidity in samples " $A$ " and " $B$ " between the fourth and the sixth months is due to the abnormal conditions under which they were kept at this time. The two samples when originally prepared were kept in 500-cubic centimeter bottles which were nearly full, but during the fourth and fifth months they were opened so frequently for the purpose of taking samples for aldehyde and peroxide tests that only about 25 cubic centimeters remained in each bottle. The increase in acidity is probably due to a continuation of the surface oxidation which is discussed in a later part of this paper. A portion of sample " $B$ " which had previously been removed to a smaller bottle showed practically no change at the end of six months.

${ }^{5}$ On further standing, however, the difference in this case is not so marked. 
in behavior between a filtered and an unfiltered oil is most pronounced during the first few months. On longer standing, the acid values tend to approach each other.

The most important fact brought out by this work is that by far the greatest deterioration which an oil undergoes takes place in the copra itself. After an oil has been expressed from the dried meat, its change on standing is very slight compared with that which is found in the same time while it is in the copra. For instance, sample No. 1 was prepared from anhydrous copra, which had stood in a closed jar during about seven months (under much better conditions than copra is ordinarily kept) ; its free acid was 1.2 per cent; an oil expressed from this copra when it was fresh had only $0.7 \%$ per cent of free acid after it had stood for seven months in tin; this same copra after remaining three weeks over water and one week more in the air yielded an oil containing 23.3 per cent free oleic acid. Samples Nos. 19 and 20 were prepared from copra which, when fresh, gave an oil with almost no free acid. Fresh coconut meat on standing for even a short time in the air becomes covered with mold and produces an oil of a more or less rancid character (cf. No. 14). No great amount of rancidity was developed in any case until signs of mold or bacterial growth were visible on the surface of the copra. From this it would seem very probable that the splitting up of fat and the accompanying "rancidity" produced in copra are in a large measure due to the action of micro-organisms, which have an excellent culture medium in the sugar, albuminoids, and water which exist, together with the oil, in coconut meat.

Koenig, Spiechermann, and Bremer, ${ }^{6}$ in their valuable paper on the decomposition of fats by micro-organisms, have conclusively shown that cottonseed meal containing a sufficient amount of water, is attacked by molds and bacteria, and that the oil therein is, on long standing, almost completely destroyed. In the accompanying experiments the methods used by these authors were followed, with certain modifications, which consisted chiefly in substituting freshly prepared anhydrous copra for cottonseed meal, and in paying especial attention to the amount of free acid developed.

The copra used for this work was prepared by grinding up fresh coconut meat and drying it at $90^{\circ}$ to $100^{\circ} \mathrm{C}$. under a partial vacuum, until the meat was anhydrous. It was then kept over sulphuric acid, to be used as needed. This product had become quite brown during the prolonged drying, but yielded an almost colorless oil, of a sweet taste, and which contained about 0.15 per cent free acid as oleic.

Ten-gram samples were weighed out in large, stoppered test tubes and each tube was inoculated with one drop of a solution made from some

\footnotetext{
${ }^{6}$ Koenig, Spiechermann, und Bremer: Beiträge zur Zersetzung der Futter- und Nahrungsmittel durch Kleinwesen. I. Die Fettverzehrenden Kleinwesen. Ztschr. f. untersuch. d. Nahrungs-u. Genussmittel (1901), 4, 721, 769.
} 
old, moldy copra. A definite volume of distilled water was then added to each, and all were allowed to stand for one week at room temperature $\left(25^{\circ}-30^{\circ}\right.$ C. $)$ and for the same length of time in an incubator at about $35^{\circ} \mathrm{C}$., the tubes being opened every morning and any change in odor or appearance noted. After two weeks the whole series was dried, weighed, the oil extracted with chloroform, and its acidity determined.

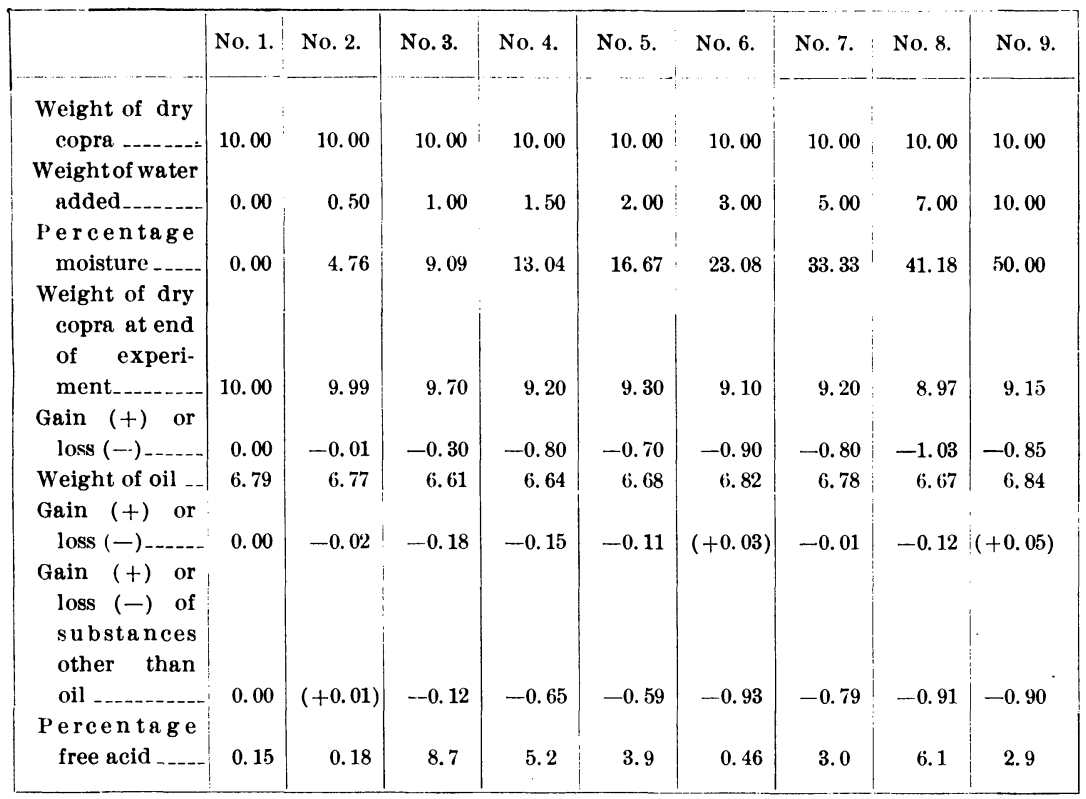

Nos. 1 and 2 remained unchanged in appearance and odor throughout the experiment. Nos. 3, 4, and 5 developed a slight mold growth and a peculiar ethereal odor, not especially unpleasant except in the case of No. 5. No. 6 showed no mold growth but turned much darker than the others and almost from the start possessed a very disagreeable odor. Nos. 7, 8, and 9 soon developed a sour smell, which, however, was not so unpleasant as that of No. 6, but they showed no mold until they had been placed in the incubator, when Nos. 7 and 8 became covered with a vigorous white growth with numerous patches of red. ${ }^{7}$ No. 9 remained unchanged in appearance.

The percentage of free acid in the oil here seems very closely to follow the appearance of a visible mold growth in the copra, being at a maximum in No. 3, where the mold first makes its appearance, decreasing slowly, with added moisture, up to No. 6 (no mold growth in evidence). increasing again in Nos. 7 and 8 (reappearance of mold growth).

As might be expected in an experiment of so short a duration, the loss in total weight of oil was in no case large, but it was sufficiently marked to show that it also chiefly took place in those tubes which contained a

${ }^{7}$ Subsequent experiments indicate that this growth in Nos. 7 and 8 was due to a loss of moisture while in the incubator: 
growth of mold; the loss of substances other than oil, on the contrary, was considerably less where the mold was most vigorous.

As nothing was known concerning the organisms with which these tubes had been inoculated, it was decided to repeat this experiment, without inoculating the tubes directly, but to start the growth by simply exposing them to the action of such organisms as might be present in the air from day to day. Therefore, the tubes were filled as before and allowed to stand at room temperature for two weeks, being opened and exposed to the air for a few minutes each day. A similar set was prepared at the same time and afterwards given to Dr. Edwards, of this Bureau, for bacteriological examination.

\begin{tabular}{|c|c|c|c|c|c|c|c|c|c|}
\hline & No. 1. & No. 2. & No. 3. & No. 4. & No. 5. & $\begin{array}{l}\text { No. } 6 . \\
\text {. }\end{array}$ & No. 7. & No. 8. & No. 9. \\
\hline $\begin{array}{c}\text { Weight of dry } \\
\text { copra }\end{array}$ & 10.00 & .10 .00 & 10.00 & 10.00 & 10.00 & 10.00 & 10.00 & 10.00 & 10.00 \\
\hline $\begin{array}{l}\text { Weight of water } \\
\text { added }\end{array}$ & 0.00 & 0.50 & 1.00 & 1.50 & 2.00 & 3.00 & 5.00 & 7.00 & 10.00 \\
\hline $\begin{array}{l}\text { Percentage mois- } \\
\text { ture }\end{array}$ & 0.00 & 4.76 & 9.09 & 13.04 & 16.67 & 23.08 & 33.33 & 41.18 & 50.00 \\
\hline $\begin{array}{l}\text { Weight of dry } \\
\text { copra at end of } \\
\text { experiment }\end{array}$ & 9.99 & 10.00 & 9.67 & 9.59 & 9.39 & 9.11 & 9.42 & 8.97 & (a) \\
\hline $\begin{array}{c}\text { Gain }(+) \text { or loss } \\
(-)\end{array}$ & $(-0.01)$ & 0.00 & -0.33 & -0.41 & -0.61 & -0.89 & -1.58 & -1.03 & \\
\hline Weight of oil & 6.83 & 6.85 & 6.24 & 6.18 & 6.54 & (6. 92 & 6. 81 & 6.83 & - \\
\hline $\begin{array}{c}\text { Gain }(+) \text { or loss } \\
(-)\end{array}$ & 0.00 & $(+0.02)$ & -0.59 & -0.65 & -0.29 & $(+0.09)$ & -0.02 & 0.00 & \\
\hline $\begin{array}{c}\text { Gain }(+) \text { or loss } \\
(-) \text { of substances } \\
\text { other than oil }\end{array}$ & $(-0.01)$ & $(+0.02)$ & +0.26 & +0.24 & -0.32 & -0.98 & -0.56 & -1.03 & \\
\hline $\begin{array}{l}\text { Percentage free } \\
\text { acid }\end{array}$ & 0.15 & 0.17 & 11.8 & 12.9 & 13.7 & 0.57 & 0.39 & 0.47 & 0.24 \\
\hline
\end{tabular}

a This tube was broken while drying.

These tubes behaved very much like those which had been used in the previous experiment, Nos. 4 and 5 showing a growth of mold and an ethereal odor in four and No. 3 in six days; No. 6 darkened and became putrid, while Nos. 7, 8, and 9 simply turned "sour."

The bacteriological examination showed no organisms to be growing in Nos. 1 and 2; bacteria were found in Nos. 3 to 9 , inclusive, and molds in Nos. 3, 4, and 5 only; the latter were much more numerous than the bacteria in Nos. 3 and 4, and about equally divided in No. 5 .

The mold most commonly occurring in Nos. 3, 4, and 5 was identified as Aspergillus flavus; others, mostly Aspergilli, were also found but as yet have not been indentified. Quite a number of bacilli were isolated in pure cultures, but no attempt at identification has yet been made. ${ }^{8}$

${ }^{8}$ Experiments are now being undertaken to study the action of pure cultures of all these organisms on copra of varying degrees of moisture. The cultures are being prepared by Dr. Edwards. The results will be published in a later paper. 
In this series of tubes, as well as in the preceding one, a high acid value, accompanied by a loss in weight of oil, is evident only in those samples which have been attacked by molds-that is, in Nos. 3, 4, and 5, which had a water content of from 9.09 to 16.67 per cent.

No. 6, the dark-colored sample with a very disagreeable odor, showed a slight gain in the weight of oil, probably due to the production, by organisms, of bodies other than oil which are soluble in chloroform. In all the other samples the weight of the oil practically remained unchanged. The large loss in substances other than oil (sugars, albuminoids, etc.) is confined, on the contrary, to those tubes in which bacteria predominate-that is, those containing more than $16.6^{\mathrm{r}}$ per cent of moisture-indicating that bacteria obtain their carbon and hydrogen chiefly from the sugars, albuminoids, and cellulose which are present in copra, while molds directly attack the oil. Whether molds alone can split up and assimilate oil from copra, or whether they may not be symbiotic with certain bacteria, remains to be established by means of the experiments to be undertaken with pure cultures.

The most important point to be considered from a practical point of view is the fact that copra containing as little as 9 per cent of moisture is still attacked by molds, with the consequent production of free acid and coloring matter as well as loss in weight of oil. Unfortunately, the copra produced in the Philippine Islands ordinarily contains from 9 to 12 per cent of water, a condition which is the most favorable for mold growth and for the deterioration of the oil. The remedy for this is obvious. A more complete drying, to reduce the water content to 5 per cent or less, will produce a copra which is unattacked by organisms. Such a product, kept dry, will remain fresh and sweet for a long time. In a previous part of this paper I have shown that copra, once sufficiently dried, may be kept during the dry season in Manila without any change whatsoever, but recent experiments prove this not to be the case during the rainy one, even with anhydrous copra.

Two samples of the latter, cut into fine pieces, were exposed, in open specimen jars, for a period of one month. At the end of this time one sample was covered to exclude air, while the other remained open. The covered sample soon developed a slight mold growth and a characteristic ethereal odor, and at the end of another month the oil extracted from it contained free acid to the amount of 3 per cent. The sample left uncovered for two months was not changed as much, for the oil from it contained only 0.89 free acid. This is probably due to the fact that during the time of exposure there occurred several comparatively dry periods of from three to four days each, during which there was very little rain, thus giving the specimen an opportunity to become partially dry so that the beginning growth of any mold would be stopped. The covered and uncovered samples were found to contain 7.8 and 6 per cent of moisture, respectively, which indicates the marked influence of a 
comparatively small amount of water on the keeping qualities of copra. As shown in the previous experiment, copra containing 4.76 per cent of moisture remains practically unchanged on standing under conditions which preclude the absorption of water, while that with 9.09 per cent produced 11.8 per cent free acid in two weeks. Between these two extremes come the two samples mentioned above, the one with 6 per cent of water increasing to 0.89 per cent and that with $\% .8$ per cent rising to 3 per cent of free acid during a period of two months.

\section{EXPERIMENTS ON COPRA DRYING.}

Since the quick and thorough drying of copra has been shown to be of such vital importance in order to insure the production of a pure oil, an investigation of various methods of copra drying has been made, taking into consideration not only the processes common in these Islands but also those which are used in other countries.

Sun drying.-As has been stated in the introduction to this series of papers, the simplest and most primitive mode of drying copra is to expose the nuts, cut in halves, to the action of the sun during about five days. This method, although it is a slow one, under favorable climatic conditions produces a very fair quality of copra. However, a sudden rainstorm or a succession of cloudy days is sufficient to start mold and bacterial growth, with the consequent deterioration of the copra. Considerable loss due to the attacks of insects and animals is also suffered during the long period of drying, and the finished product very seldom contains less than 9 per cent of moisture.

Grill drying.-A much quicker method is the one carried out by laying the half nuts, face downward, on a bamboo grating placed over a slow fire of coconut husks. After being dried in this manner over night the nuts are removed from their shells and are then again placed over the fire, where they are allowed to remain for from four to five hours longer. This process, although it is cheap and comparatively rapid, has the disadvantage of yielding a dark-colored product which has a smoke-like taste and odor, and it also tends to form a hard, burnt coating over the surface of the nut while the inside is left in a comparatively moist state, a fact which is often taken advantage of by the small producers, who sell their copra by weight. Commercial copra prepared in this way contains from 9 to 13 per cent of moisture.

Hot-air drying.-This method of desiccation has been used successfully for a long time in the preparation of coffee, cacao, dried fruits, etc., and is at present in quite extensive use for the making of copra in Ceylon, ${ }^{\mathbf{0}}$ where it is said to give a very pure, light-colored product. The type of apparatus used in that island essentially consists of a large chamber filled with wire trays upon which the coconuts are placed and over which a

${ }^{9}$ Tropical Agriculturalist, 23, No. 10, supplement. 
current of hot air, driven by a fan, is passed. In Trinidad, ${ }^{10}$ British West Indies, there is now in operation a rotary hot-air drier which, it is stated, is better than any other apparatus now in use.

For the purpose of testing the efficiency of the stationary form of hot-air drier, a double-walled, rectangular galvanized-iron box, having an internal capacity of about 0.2 cubic meter, was constructed. Three galvanized-iron trays, perforated at one end, were set in this box in such a manner that the stream of hot air entering through a 20-centimeter pipe at the bottom was compelled to pass over each in turn before escaping at the top of the apparatus. A constant current of air was obtained by means of a small electric fan which was connected with a section of 15-centimeter pipe, so arranged that it could be heated by a small kerosene stove to any desired temperature. The apparatus had a maximum capacity of 24 nuts split in halves or 12 nuts when shredded.

Experiment I.-Four nuts were split in halves and placed on the bottom tray.

Temperature of entering air, $56^{\circ} \mathrm{C}$.

Temperature of escaping air, $51^{\circ} \mathrm{C}$.

Time of drying, 20 hours.

The copra dried at this comparatively low temperature was very white and of the best quality. A sample of oil expressed from it contained 0.08 per cent free acid.

Experiment $I I$. - The meat from twelve nuts was shredded by hand and treated for one day in the same manner as in the preceding experiment; it was then allowed to stand at room temperature over night and completely dried on the following day. The substance in the bottom tray naturally desiccated much more rapidly than in the other two, therefore as soon as one tray was completely dry it was removed and replaced by the one just above it.

Temperature of entering air, $56^{\circ} \mathrm{C}$.

Temperature of escaping air, $50^{\circ} \mathrm{C}$.

Actual time of drying:

Top, $14 \frac{1}{2}$ hours.

Middle, $12 \frac{1}{2}$ hours.

Bottom, $9 \frac{1}{2}$ hours.

The less completely dried copra in the two upper trays became slightly "soured" while standing over night. This caused a slight increase in free acid as follows:

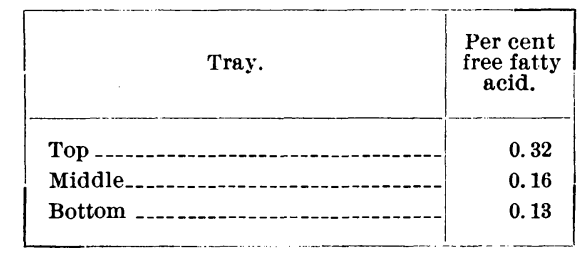

${ }^{10}$ Journal d'Agriculture Tropicale (1904), 103. 
Experiment III.-The meat from four nuts was shredded and placed in the bottom tray, being stirred every half hour.

Temperature of entering air, $93^{\circ} \mathrm{C}$.

Temperature of escaping air, $74^{\circ} \mathrm{C}$.

Time of drying, $3 \frac{1}{2}$ hours.

The copra thus produced was thoroughly dry, very white, and pleasant to the taste. The oil expressed from it contained only 0.06 per cent free fatty acid.

In Experiment II, as would naturally be expected, it is evident that the meat farthest away from the entering air requires a much longer time for drying than does that which lies closer to the bottom of the box. This is due to the fact that the air gradually becomes cooler and more completely saturated with water vapor as it passes over the moist copra. For practical use, therefore, a drier should be equipped with some sort of a mechanical carrier which would constantly introduce fresh coconut meat at the coolest part of the machine and then bring it slowly down toward the hottest portion.

Experiment $I V$.- This was undertaken in an endeavor to ascertain the approximate time required completely to dry the fresh meat, introducing it at the top of the apparatus and shifting it gradually toward the bottom. Four trays, each containing the freshly grated meat of 4 coconuts, were prepared, and three of these were placed in the drier simultaneously, tray No. 1 being at the bottom. After the latter had become sufficiently dry, it was removed from the apparatus and tray No. 2 moved down to take its place; this was next replaced by No. 3, and finally in the same manner by the moist sample No. 4 .

Entering air, $95^{\circ} \mathrm{C}$.

Escaping air, $70^{\circ} \mathrm{C}$.

Actual time of drying:

No. $1,4 \frac{1}{2}$ hours.

No. $2,5 \frac{1}{2}$ hours.

No. $3,6 \frac{3}{4}$ hours.

No. 4,4 hours.

From the above experiments it may be concluded that the average time of drying, where the apparatus is run continuously at $95^{\circ} \mathrm{C}$., will approximately be four hours.

The rotary drier.-A section of galvanized-iron pipe 20 centimeters in diameter by 6 meters long was set up on wheels and connected with a small electric motor so that it could be made to revolve at any desired speed. The same current of hot air which was previously used for the stationary drier was connected with this apparatus. Four strips of angle iron extending throughout the length of the pipe served to keep the moist copra in constant motion during the time of drying. After much preliminary work to determine the proper inclination necessary to allow the material to pass through the apparatus with sufficient slowness, it was 
found that by careful manipulation the grated meat from four nuts could be dried in about two hours so as not to contain more than 6 per cent of moisture. The only objection to this method consists in the difficulty of regulating the speed with which the ground meat passes from one end of the apparatus to the other. This is dependent on four factors: (1) The number of revolutions per minute, (2) the angle of inclination, (3) the specific gravity of the coconut meat, and (4) the speed of the entering current of hot air. In the machine used here an unfortunate tendency toward a separation of the moist from the dry copra appeared; the dry particles, being lighter, were held back by the current of air or even blown out through the upper end of the tube, whereas the moist and consequently heavier pieces passed through too quickly. When these mechanical difficulties are solved this should prove the ideal method for drying coconut meat for oil-making purposes.

Vacuum drying.-The apparatus used was a small, barrel-shaped iron chamber, about 34 centimeters in diameter and in length, insulated with asbestos and heated by three hollow steam plates upon which the substance to be dried was placed. The pump connected with this drier gave a vacuum of about 660 millimeters (absolute pressure of 100 millimeters).

Experiment I.-Four coconuts (the maximum capacity of the apparatus) were split in halves, after removing the outer husk, and kept in the drier for three hours. The meat had then contracted sufficiently to allow of its being removed from the shell. During this time the temperature had gradually risen from $30^{\circ}$ to $80^{\circ}$. The meat was then subjected to a further drying during four hours, at the end of which time, though not perfectly anhydrous, it was fully as dry as the ordinary commercial article.

Actual time of drying, 7 hours.

Maximum temperature, $80^{\circ} \mathrm{C}$.

Vacuum, 635 millimeters.

Steam pressure, about 0.7 kilo per square centimeter ( 10 pounds).

Experiment II.-The preceding experiment was repeated under practically the same conditions, except that the nuts were allowed to dry completely without removing the shell.

Time of actual drying, 8 hours.

Maximum temperature, $80^{\circ} \mathrm{C}$.

Vacuum, 648 millimeters.

Steam pressure, about 0.7 kilo pér square centimeter ( 10 pounds).

Experiment III.-An attempt was made to shorten the time of drying by increasing the steam pressure and having the machine hot before putting in the nuts, the initial temperature being $75^{\circ}$.

Actual time of drying, $5 \frac{1}{2}$ hours.

Maximum temperature, $85^{\circ} \mathrm{C}$.

Vacuum, 640 millimeters.

Steam pressure, about 4.2 kilos per square centimeter ( 60 pounds). 
Experiment $1 V$.-The meat from four nuts was ground and spread in a layer of a depth of about 3 centimeters in shallow glass dishes. The initial temperature was $60^{\circ}$.

Actual time of drying, 9 hours.

Maximum temperature, $75^{\circ}($.

Vacuum, 660 millimeters.

Steam pressure, 0.7 kilo per square centimeter (about 10 pounds).

Experiment $V$.--Four coconuts were split in halves and put into the machine, the latter being left partly open and with no vacuum.

Actual time of drying, 11 hours.

Highest temperature, $86^{\circ} \mathrm{C}$.

Steam pressure, 0.7 kilo per square centimeter (about 10 pounds).

'Therefore, under the best conditions obtainable (temperature $85^{\circ}$ and vacuum 635 to 660 millimeters), the minimum time required for vacuum drying was five and one-half hours. ${ }^{11}$

If we are to form our judgment from the great efficiency of the vacuum evaporators used for sugar solutions and for many other liquids, it might be supposed that this process would be equally advantageous for coconuts. However, the two conditions are altogether different. In the case of solutions we have a thin layer of liquid in direct contact with a heated surface, the evaporation taking place so rapidly that the space above the liquid is constantly saturated with moisture; the main object of these machines is to remove and condense the surplus water vapor as rapidly as possible and by so doing to allow the evaporation to proceed at a comparatively low temperature. The water in coconut meat, on the other hand, which at the most is not greater than 50 per cent of the total weight of material, under the best conditions, diffuses very slowly through the cells of the copra to the surface, the removal of moistureladen air therefore becoming a matter of secondary importance. The principal consideration is the constant application of as much heat to the entire surface of the material as the latter can endure without becoming burnt. That this condition is not fulfilled in the best manner by a vacuum drier is chiefly due to the poor conductivity of the rarified air which it is necessary to heat. Although the temperature of the steam plates in the drying oven is from $100^{\circ}$ to $110^{\circ}$, that of the partial vacuum immediately above and surrounding the copra, even after several hours, rarely rises above $75^{\circ}$. To this local superheating for a long period of time at the point of contact with the plates, probably is due the brown color and slightly burnt taste which vacuum-dried copra almost invariably possesses.

For the sake of comparison I append the following table showing the

${ }^{11}$ This does not include the time necessary to produce steam and to heat up the drier. These items must be considered unless the apparatus is to run continuously. 
approximate time required to dry copra under the most favorable conditions by each of the methods previously considered:

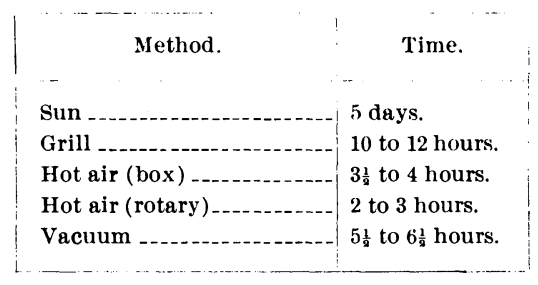

The quality of the copra produced by the hot-air box drier is very much superior to that yielded by any other method, since it is perfectly white and dry, retaining the pleasant odor and taste of fresh coconut meat. For oil-making purposes the rotary apparatus, because it lends itself to a continuous process and requires considerably less time, recommends itself especially, although its product does not present quite so pleasing an appearance. Either of these two methods, on account of their cheapness and simplicity, should be preferred to vacuum drying.

Centrifugating.-Another method of drying suggests itself, which should prove to be very efficient, although, owing to lack of facilities, I have not as yet been able to give it a practical test. This is to extract the meat from coconuts by means of a rotary burr and to run this product directly into a powerful centrifugal from which the greater part of the water would be thrown off at once. A comparatively short, supplementary drying by means of hot air would then suffice to prepare copra for expressing the oil. Another point in favor of this method is that the copra resulting therefrom, having lost most of its sugar and albuminoids together with its water in the process of centrifugation, would be able to withstand a higher temperature while drying (with a resulting economy of time) without showing the same tendency to turn brown. Once dry, it could be stored with less danger of deterioration through mold action than material prepared by ordinary methods. The objection may be raised that, during the centrifugation, a considerable amount of oil together with the water would be thrown off from the fresh meat, and that this would either entirely be lost or would necessitate much labor for its recovery. This, to a certain extent, is true, as the water in coconut meat exists in the form of a cream-like emulsion with oil, sugar, and albuminoids. A sample of this "coconut cream," prepared by expressing the fresh meat in a hand press, was, on analysis, found to have a specific gravity of 1.012 at $30^{\circ} \mathrm{C}$. and to consist of-

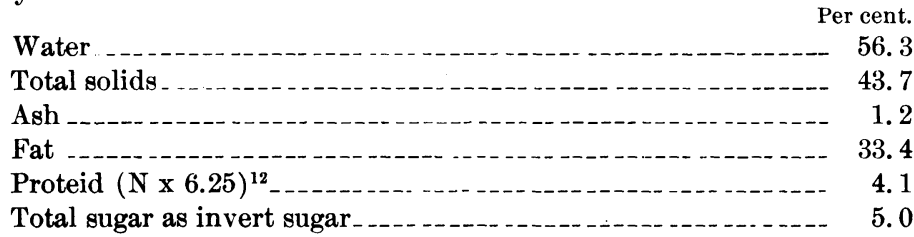

${ }^{12}$ Determination made by Mr. Richmond, of this laboratory. 
The above results show that it approximates in nutritive properties the composition of a rich, natural cream; it is very pleasant and sweet to the taste, possesses an agreeable odor, and, when sterilized and properly sealed, will remain indefinitely in a fresh condition. Such a product could be used as as substitute for all of the purposes to which the socalled "evaporated creams," now on the market, are put, and it might prove to be one of the most valuable by-products of the coconut-oil industry.

THE ACTION OF ORGANISMS ON COCONUT OIL UNDER VARYING CONDITIONS.

Although, as has been shown above, the character of a coconut oil in regard to free acid, odor, and taste is determined chiefly by the quality of the copra used for its production, there is also in most commercial oils a slow but steady deterioration, amounting in the worst cases to a rise of about 0.5 per cent per month (cf. sample No. 31, p. 120), while with pure, filtered oils this reaches only a few hundredths per cent in the same time. It has been remarked above that samples of oil which contain suspended impurities and water, as a rule, increase in their content of free acid somewhat more rapidly than do similar ones which have been clarified; a result to be expected, if, as is the case with copra, decomposition is due to micro-organisms, since it has been proven that bacteria and molds do not live for any length of time in pure oil. ${ }^{13}$

The influence of impurities on the keeping qualities of oils was noticed as early as 1855 by Pelouze, ${ }^{14}$ who observed that various oleaginous seeds, when crushed and extracted at once, yielded almost neutral oils, whereas if, after being crushed, they were allowed to stand for some time before extraction, the oil then produced contained a large amount of free acid. He considered this action to be due to a "ferment" similar to that producing alcohol from sugar.

Pastrovich and Ulzer ${ }^{15}$ using a mixture of oleomargarine with 0.5 per cent casein and 1 per cent water, observed an increase of acidity from 0.888 to 1.259 per cent in one week, and in fourteen weeks 0.888 to 10.270 per cent. They make no attempt to explain this effect, evidently attributing the saponification to some change brought about directly by the presence of albuminoids, although it is very probable that it was produced by bacteria or molds.

The following experiments were undertaken with a view of accentuating this difference in keeping qualities between pure and impure oils by exposing them directly to the action of micro-organisms under similar conditions.

About 20 cubic centimeters each of samples Nos. 6, 8, and 11 were poured into small beakers and placed in a covered specimen jar containing

${ }^{13}$ E. Ritsert: Untersuchungen über das Ranzigwerden der Fette. Chem. Centrb. (1890), 507, 575, 813 .

${ }^{14}$ M. J. Pelouze: Memoir, sur la saponification des huiles sous l'influence des matieres qui les accompagnent dans les grains. Compt. Rend. (1855), 40 ; 605 .

${ }^{15}$ Pastrovich $u$. Ulzer: Ueber den Einfluss der Gegenwart verschiedener Eiweisskörper auf Fette. Ber. d. chem. Gesell. (1903), 36, 209. 
a little moldy copra together with a beaker of water to insure an abundance of moisture. They were then allowed to stand at room temperature $\left(25^{\circ}\right.$ to $30^{\circ}$ C.) for two months. Their change in acid value is shown as follows:

\begin{tabular}{|c|c|c|c|c|}
\hline \multirow[b]{2}{*}{ Number of oil. } & \multicolumn{2}{|c|}{$\begin{array}{l}\text { After standing one } \\
\text { month. }\end{array}$} & \multicolumn{2}{|c|}{$\begin{array}{l}\text { After standing two } \\
\text { months. }\end{array}$} \\
\hline & $\begin{array}{l}\text { F. F. A. } \\
\text { in oil } \\
\text { exposed to } \\
\text { mold. }\end{array}$ & $\begin{array}{l}\text { F. F. A. } \\
\text { in oil in } \\
\text { original } \\
\text { bottles. }\end{array}$ & $\begin{array}{l}\text { F. F. A. } \\
\text { in oil } \\
\text { exposed to } \\
\text { mold. }\end{array}$ & $\begin{array}{l}\text { F. F. A. } \\
\text { in oil in } \\
\text { original } \\
\text { bottles. }\end{array}$ \\
\hline (3 - - - & 0.22 & 0.16 & 0.26 & 0.17 \\
\hline 8 & 0.23 & 0.14 & 0.26 & 0.17 \\
\hline 11 - & 0.22 & 0.18 & 0.83 & 0.22 \\
\hline
\end{tabular}

A slight increase of acidity was evident in each of these samples, but No. 11, an unfiltered, very turbid oil, was decomposed much more rapidly than either of the others. It also was characteristically rancid in odor and taste and contained a visible mold growth.

Since a marked difference was shown in the behavior of these oils, it was decided to observe the effect of the addition of small quantities of nutrient matter and of water on the rise in the free-acid contents of a pure oil. The nutrient material which was used was prepared from "latic," a coagulated residue produced in the native process of making oil, by boiling down an emulsion of fresh coconut meat. This residue, when dried and extracted with chloroform, yields a light-brown powder, partially soluble in water and of a sweetish, not unpleasant, taste. It consists chiefly of albumin and sugar. The following samples were prepared, using pure, fresh oil as a base:

A. 25 cubic centimeters oil +0.25 grams "latic."

B. 25 cubic centimeters oil +0.25 grams "latic" +0.25 cubic centimeters water.

C. Control of pure oil.

Each of these samples was placed in a 50-cubic centimeter glass bottle inoculated with one drop of the moldy oil (No. 11) used in the previous experiments, and allowed to stand for one week at room temperature, and, after determining the increase in acidity, for one week in the incubator at $35^{\circ}$. Finally, the acidity was tested after the oil was allowed to stand for two months longer at room temperature.

\begin{tabular}{|c|r|c|c|c|}
\hline Sample. & $\begin{array}{c}\text { Initial } \\
\text { acidity. }\end{array}$ & $\begin{array}{c}\text { One } \\
\text { week at } \\
\text { room } \\
\text { tempera- } \\
\text { ture. }\end{array}$ & $\begin{array}{c}\text { One } \\
\text { week at } \\
35^{\circ} \mathrm{C} .\end{array}$ & $\begin{array}{c}\text { Two } \\
\text { months at } \\
\text { room } \\
\text { tempera- } \\
\text { ture. }\end{array}$ \\
\hline$A$ & 0.10 & 0.11 & 0.13 & 0.27 \\
$\mathrm{~B}$ & 0.10 & 0.25 & 0.69 & 3.8 \\
$\mathrm{C}$ & 0.10 & 0.10 & 0.11 & 0.13 \\
\hline
\end{tabular}


The following table gives the results of a similar experiment in which 10 cubic centimeters of the oil to be tested were inoculated with a drop of moldy oil, then poured out in a Petrie dish, which was placed in a closed specimen jar over a little moist, moldy copra, and allowed to stand in the incubator at $33^{\circ}$ to $35^{\circ} \mathrm{C}$. for one week:

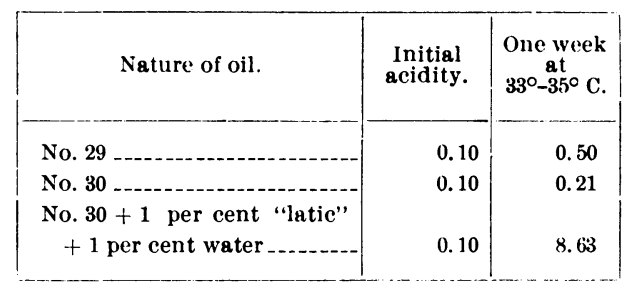

The last sample, which showed the greatest increase in acidity I have yet been able to produce in a short time, was almost completely covered by a greenish-yellow mold, similar to that noticed on copra, and it had the characteristic odor of a rancid oil.

In the light of these latter experiments there can be no doubt of the fact that coconut oil, provided it contains sufficient moisture and nutrient matter, is attacked by micro-organisms, principally molds, with an accompanying production of free acid and of a disagreeable taste and odor. This is the principal cause of "rancidity" in coconut oil, if by "rancidity" we mean a high acid content and a bad taste and odor. Whether this fat splitting is directly due to a life process of the molds or to an enzyme secreted by them is a problem which has not yet been solved. However, it seems highly probable that these molds produce a slowly acting enzyme, soluble in oil, which continues its hydrolytic action even after the organisms themselves are dead. This would account for the steady increase in free acid of some commercial oils which are perfectly clear and free from impurities and which have been proven to contain no living bacteria or molds. Experiments are now being carried on to clear up this point.

\section{OTHER FACTORS INFLUENCING THE ACIDITY OF OIL.}

Effect of sunlight.-Twenty-five cubic centimeters each of samples "A" and "11" (see table, p. 119) were placed in 50-cubic centimeter glassstoppered bottles and allowed to stand in the sun for one month. At the end of this time " $\mathrm{A}$ " contained 0.22 per cent and " 11 " 0.24 per cent of free acid, while at the same time the original samples. " $\mathrm{A}$ " and " 11 " showed 0.06 and 0.18 per cent, respectively. No marked change in taste or odor could be detected. The acid content of the pure sample "A" appears to have increased considerably more than that of "11," due probably to its contamination with a few drops of water during a hexvy rain. However, the total amount of acid developed was so small that the experiment was not repeated.

Effective of heat and moisture.-Several samples of oil were heated at $100^{\circ}$ for periods up to twenty-four hours with no change in acid 
value. However, on heating in a sealed tube, considerable alteration took place, as is shown in the following: Samples from No. "B" and from that portion of No. 11 which had been exposed to sunlight were heated in sealed tubes at about $160^{\circ}$ for ten hours. " $\mathrm{B}$ " changed in percentage of free acid from 0.06 to 0.90 , but was unchanged in color, odor, or taste. " 11 " rose from 0.24 per cent to 2.05 per cent under the same conditions, and possessed a very disagreeable odor and a nauseating taste. It also showed a considerable increase in color, probably due to decomposed albumin, etc. The change taking place in the sample was demonstrated to be a simple hydrolysis by heat and moisture. In the next experiment, both "B" and " 11 " (original samples) were dried very carefully by passing through them a current of dry air for seven hours at $100^{\circ} ; 10$ cubic centimeters each of the dry oils were then sealed in glass tubes and heated at about $160^{\circ}$ for ten hours. For comparison, two more tubes of oil were subjected to the same conditions, one containing 10 cubic centimeters of sample 11 (undried), the other 10 cubic centimeters of the dried oil " $\mathrm{B}$ " plus 3 drops of water.

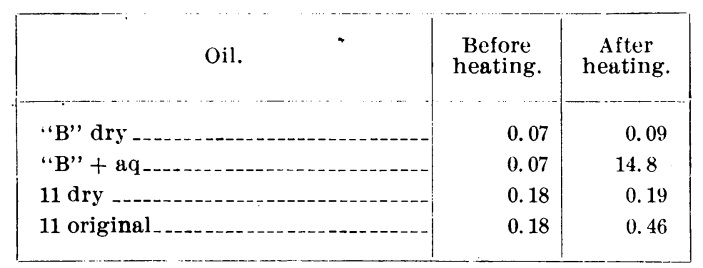

In neither of the samples marked " $\mathrm{B}$ " was a bad odor or any color produced, though the hydrolyzed sample was slightly unpleasant to the taste, owing to the large amount of free acid present. However, both the similarly treated tubes containing oil No. 11, in spite of their low acid value, were decidedly disagreeable to the taste and smell and presented a decomposed appearance.

\section{FACTORS WHICH CAUSE RANCIDITY IN OIL.}

The average person, if asked to judge of the quality of these four heated oils by the sense of taste and smell alone, would almost invariably say that both samples of oil No. 11 were "rancid" and that the other two were fairly pure, whereas, judging from the amount of free acid present, one might consider " $\mathrm{B}+$ aq." as the only sample containing a marked amount of rancidity. From the above it is evident that rancidity and free acid are not by any means synonymous and that the cause of the former must be sought elsewhere than in the percentage of the latter.

Lewkowitsch applies the term "rancidity" only to those fats which contain an excess of free fatty acids due to the action of air.

Alder Wright ${ }^{18}$ gives a resumé of the work done on this subject and concludes 168.

10 Alder Wright: Fixed Oils, Fats, Butters, and Waxes, second edition (1903), 
that rancidity is the alteration which oils and fats undergo when not protected from the influence of air and light. "Such oils," he says, "acquire a sharp, disagreeable taste and odor, their proportion of free acids gradually increases, and they undergo various other chemical alterations."

Schmid"17 differentiates between "sour fats," "rancid fats," and "sour and rancid fats." "A fat is sour," he says, "when its content of free fatty acids is abnormally high but the free glycerine is unchanged. A fat is rancid when the proportion of free fatty acids is not high but the free glycerine has been oxidized partially or completely to aldehydes and ketones. $\mathbf{A}$ fat is rancid and sour when it contains a large amount of free acid together with oxidation products of glycerine." As a test for rancidity he proposes a 1 per cent solution of m. phenylendiamin.

Scala ${ }^{18}$ found œnanthylic aldehyde among other substances present in rancid olive oil, and assumes that this body gives the characteristic odor and taste termed "rancidity."

Bianchi ${ }^{19}$ proposes, as a test for rancidity, to shake up a little of the oil in question with fuchsin-sulphurous acid, a violet-red color indicating rancidity.

Brown ${ }^{20}$ uses this same test in the study of butter fat, and presumes that the rancid odor is due to acroleïn. Various other tests for rancidity have been proposed, all depending on the presence of aldehydes.

The most satisfactory, in my experience, is that with fuchsin-sulphurous acid, shaking up about equal parts of oil and reagent. Nearly all the samples of coconut oil prepared in this laboratory, after standing for several months, responded to this test, but they gave, not a violet red, as has been stated to be the case with other rancid oils, but a more or less blue coloration with only a slight tinge of red. The above review of the literature will demonstrate that the causes of rancidity are by no means clear. Certainly œnanthylic aldehyde has not an intensely disagreeable odor; acroleïn has, but then it gives a red and not a blue color with fuchsin-sulphurous acid.

\section{ACTIVE OXYGEN IN COCONUT OIL WHICH HAS BEEN STANDING.}

Another peculiarity of pure coconut oil is that, after it has been standing exposed to light and air for a few months, it almost invariably contains active oxygen. Five cubic centimeters of sample "A," shaken in an Ehrlenmeyer flask with a mixture of 50 cubic centimeters of water, 5 cubic centimeters glacial acetic acid, and 1 gram potassium iodide and allowed to stand for one hour, produced a deep-yellow coloration in the water solution, requiring 0.25 cubic centimeter $\frac{N}{10}$ sodium thiosulfate for decolorization. A blank test, with freshly prepared oil, remained perfectly colorless during the same time. A simple way of performing this test is to saturate a strip of starch iodide paper with the oil in question,

\footnotetext{
${ }^{17}$ A. Schmid: Zur Prüfung der Fette auf Ranzidität. Z. Anal. Ch. (1901), 37, 301.

${ }^{18}$ Alberto Scala: Staz. sper. Agrar. ital, 30, 613, Centrbl. (1898), 439.

${ }^{19}$ Centrbl. (1898), II, 948.

${ }^{20}$ Brown: The Chemistry of Butter Fat. Jour. Amer. Chem. Soc. (1899), 21, 975.
} 
place it on a glass plate, and carefully add one drop of 10 per cent acetic acid. When old oil is used, a blue ring appears around the drop of acid in from one to five hours, whereas a freshly prepared sample remains uncolored for twenty-four hours or more, provided the test paper is kept under a bell jar to exclude laboratory fumes. This reaction is at least as delicate as any of the tests for rancidity based on the presence of aldehyde. Whether it is given by other rancid oils can not be stated at present, for I have as yet failed to find in the chemical literature any mention of such oxidizing substances in oil. The following is a table showing the reaction of our samples of coconut oil with fuchsin-sulphurous acid and with starch iodide, together with their age and acidity at the time of testing. For the previous history of these oils, see page

\begin{tabular}{|c|c|c|c|c|}
\hline No. & $\begin{array}{l}\text { Approxi- } \\
\text { mate age, } \\
\text { in months, } \\
\text { at time of } \\
\text { testing. }\end{array}$ & $\begin{array}{l}\text { Approxi- } \\
\text { mate per- } \\
\text { centage } \\
\text { F. F. A. }\end{array}$ & $\begin{array}{l}\text { Color with fuchsin-sulphurous } \\
\text { acid. }\end{array}$ & $\begin{array}{l}\text { Test } \\
\text { for perox- } \\
\text { jdes. }\end{array}$ \\
\hline $\mathbf{A}_{-}$ & 15 & 0.1 & Strong blue & $+\mathbf{s}$ \\
\hline $\mathbf{B}_{-1}$ & 5 & 0.1 & - & $+a$ \\
\hline 1 & $5 \frac{1}{2}$ & 1.5 & - - do - - & + \\
\hline 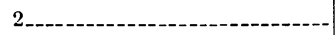 & $5 \frac{1}{8}$ & 1.5 & - & + \\
\hline 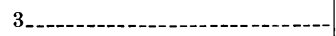 & $5 \frac{1}{2}$ & 2.1 & - - - do _- - - - - - - & + \\
\hline 4 & $5 \frac{1}{8}$ & 5.9 & - & + \\
\hline 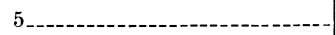 & $5 \frac{1}{2}$ & 7.6 & Blue-red & - \\
\hline $\boldsymbol{b}_{-}$ & $5 \frac{1}{2}$ & 0.2 & Strong blue & Trace. \\
\hline 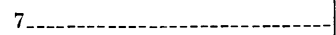 & $5 \frac{1}{2}$ & 0.2 & - & + \\
\hline \& 8 & $5 \frac{1}{2}$ & 0.2 & - & + \\
\hline $9+-1-0-1-1$ & $5 \frac{1}{2}$ & 0.2 & - & + \\
\hline 10 & $5 \frac{1}{2}$ & 0.2 & - & + \\
\hline 11 & 5 & 0.3 & - do & + \\
\hline 12 & 5 & 0.1 & - & + \\
\hline 13 & 5 & 0.1 & - do & + \\
\hline 14 & 5 & 4.0 & No color & Trace. \\
\hline 15 & 5 & 0.9 & Strong blue & + \\
\hline 16 & 5 & 2.0 & - & + \\
\hline 17 & 4 & 0.1 & 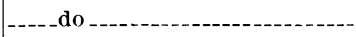 & + \\
\hline 18 & 4 & 0.2 & - do & + \\
\hline 19 & 4 & 1.2 & - do & + \\
\hline 20 & 4 & 0.7 & No color & t- \\
\hline 21 & 4 & 0.7 & - & + \\
\hline 22 & $3 \frac{1}{2}$ & 23.3 & Red --1- - & - \\
\hline 23 & $3 \frac{1}{9}$ & 1.6 & Light blue & Trace. \\
\hline 24 & $3 \frac{1}{2}$ & 1.5 & Strong blue & + \\
\hline 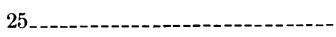 & $3 \frac{1}{2}$ & 3.4 & Moderate blue & + \\
\hline 26 & $3 \frac{1}{2}$ & 2.6 & Strong blue & + \\
\hline 27 & $3 \frac{1}{2}$ & 2.4 & Light blue & Trace. \\
\hline 28 & $3 \frac{1}{2}$ & 3.5 & Very strong blue & + \\
\hline 29 & 2 & 0.1 & No color & + \\
\hline $30-1-1--1$ & 2 & 0.4 & Light blue & + \\
\hline 31----1---- & $3 \frac{1}{2}$ & 3.5 & Trace & + \\
\hline 32 & $3 \frac{1}{2}$ & 7.8 & Moderate blue & - \\
\hline 33 & 7 & 7.0 & No color & - \\
\hline 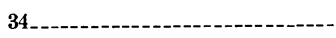 & $5 \frac{1}{9}$ & 10.2 & Strong blue & + \\
\hline 35-1-1-1-- & $\frac{1}{9}$ & 5.0 & No color & + \\
\hline
\end{tabular}

Very strong. 
In exanining this table it will be noticed that in almost every case a distinct blue color with Schiff's reagent is accompanied by a positive test for peroxide, and, in the two cases where a red coloration predominates, the peroxide test is negative. 'The strongest tests for both peroxide and aldehyde were given by samples $\mathrm{A}$ and B-two very pure oils-while, contrary to our expectations, the commercial oils as a rule either failed to respond entirely or gave very weak tests, though they were infinitely worse than the pure samples in every other particular. 'The only change noticeable in samples $\mathrm{A}$ and $\mathrm{B}$, on standing, was the development of a peculiar, pungent, "strong" odor and a slight burning "after taste ;" otherwise they were practically the same as when freshly prepared. Just what this "strong" odor in pure oils is caused by can not at present be stated, although the subject is now being investigated. It can hardly be caused by either œnanthol or acroleïn, as both these substances, when mixed with oil, give a red and not a blue color with Schiff's reagent. Glycerine aldehyde ${ }^{21}$ under certain conditions produces a blue-red coloration with this test, but it has no odor. However, the process by which this change is produced is undoubtedly due to direct oxidation by light and air, since bacterial or mold action may be excluded in the case of a pure oil. That it is largely a surface action is indicated by the facts that (1) samples A and $\mathrm{B}$, which were kept in large bottles, about half full, have deteriorated in five months to a much greater extent than have other samples of pure oil which were kept in small, nearly full bottles for over a year; (2) both the aldehyde and peroxide tests were given by samples of fresh oil which were exposed to the air on strips of filter paper for one or two weeks ;2 $^{2}$ (3) the same effect can be produced by treating fresh oil with platinum black for a few hours, or by heating it, exposed in a thin layer, to $100^{\circ}$ for ten or twelve hours.

A possible explanation of this production of rancidity in pure oils is that a small percentage of fatty acid is oxidized to an oxyacid, which in turn forms a lactone, and (assuming the formation of hydrogen peroxide) the latter would give rise to a peracid, which, in turn, would oxidize the free glycerine to an aldehyde. The absence of peroxide and, as a rule, of aldehyde in commercial coconut oils, or in those purposely subjected to the action of micro-organisms, may be due to the presence of sugars and other reducing substances commonly present in the impure oils, or to the fact that the glycerine set free by mold action is completely oxidized to carbon dioxide and water. The nonexistence of free glycerine in highly rancid fats has been noted by Sparth ${ }^{23}$ and other observers. This question of the products arising from the oxidation of pure coconut oil by air is now being taken up more thoroughly, and the results will be published in a later paper. However, from a commercial point of view, it is of

${ }^{21}$ E. Fischer u. Tafel. Ber. d. Chem. Gesell. (1887), 20, 3384.

${ }^{22}$ Freer \& Novy: Amer. Chem. J. (1902), 27, 161.

${ }^{23}$ Zt. Anal. Ch. (1896), 35, 471. 
comparatively little importance, when one considers the marked deterioration produced by micro-organisms acting on copra and impure oil. If stored in nearly air-tight containers very little if any oxidation should take place even on long standing or on transportation. 'The main points to remember are that the copra from which oil is made should be fresh and be prepared under as good conditions of drying as possible and the oil should be thoroughly dried and filtered until absolutely clear. If properly prepared, it should then be capable of shipment without noticeable deterioration. It is obvious that the best results will be obtained by expressing the oil in the country in which copra is dried, and by using the best machinery for preparing the latter.

\section{SUMMARY.}

Soil.--In attempting by means of soil analyses to explain why coconut trees growing near the seashore are more prolific than those planted farther inland, it was observed that-

(1) Chemically, there is very little difference in soils from the two localities, those from inland regions being, if anything, a little more fertile.

(2) The salt water from the sea has no influence on trees in its vicinity, as only amounts of chlorine so small as to be negligible were found to be present even at the bases of coconut trees which were actually growing on the beach.

(3) The greater porosity of soils near the sea, coupled with the fact that they are, as a rule, practically saturated with water at a distance of only a few feet beneath the surface of the ground, is the principal reason why they are more suitable for trees like the coconut, which require an enormous quantity of water for their growth.

(4) Although good coconut soils are apparently almost devoid of fertility, yet, taking into account the character of coconut roots and the large area from which each tree draws nourishment, it can be demonstrated that there exists an ample supply of nutriment for their growth.

The nut; age in reference to quality.-(1) The variations among individual nuts is sufficiently great to render exact conclusions from analytical data difficult, but, taking the average of a number of determinations, there appears to be a slight increase in the proportion of meat, copra, and oil in nuts which have been stored up to a maximum time of three months after cutting. Beyond this period there is a decided decrease in these constituents. Nuts taken from the same tree show somewhat less individual variation.

(2) Four series of ten nuts each, of varying degrees of ripeness, showed a marked difference in the amount of copra and of oil to be obtainable from them, the percentage of the oil in a green nut being only about one-half of that which it is when the nut is fully ripe. This ripening process continues to some extent, on storage, after cutting. 
(3) Analyses of coconuts from the same locality, but having husks of different color, prove that the color of a nut has very little if any influence on its composition.

(4) 'The difference between trees noar the seashore and those farther inland is solely in the quantity, not in the quality, of nuts which they produce, coconuts from inland regions averaging fully as well as those from the beach. This fact is shown both by analyses and by practical tests on a large scale.

(5) Analyses were made of twenty ripe coconuts from Davao, Mindanao, and they were found to have very much the same proportion of the various constituents and to give the same total yield of oil as the average lot of ripe nuts from San Ramon.

(6) Coconut oil is generally stated to have a great tendency to become rancid, but all the experiments made in this laboratory show that, when once prepared in a pure state, its keeping qualities are equal if not superior to those of most other vegetable fats and oils. This popular fallacy in regard to coconut oil probably arose from the inability or disinclination on the part of most observers to procure pure samples, as the commercial product unquestionably has a high acid value and a bad odor, and deteriorates with fair rapidity, this change being greater as a rule the greater the initial acidity of the oil.

(7) Most of the free acid and the accompanying bad odor and taste is produced in the copra itself before the oil has been expressed. The oil from a sample of copra which had been cut into fine pieces and exposed to moist air for one month increased in acidity from 1.5 to 23.3 per cent.

(8) The hydrolysis and subsequent destruction of fat in copra is brought about by molds (the greater part of which are Aspergilli), acting either alone or in symbiosis with certain bacteria, the condition most favorable to this growth being a moderately high, constant temperature and a water content of from about 9 to 17 per cent. No organisms were found growing on a sample containing $4 . \% 6$ per cent of moisture and no change in acidity took place. Samples containing from 23 to 50 per cent of water were infested by several species of bacteria which subsisted on the nonfatty portion of the copra but produced very little free acid from the oil. No molds were found in these samples.

(9) Ordinarily, commercial copra contains from 9 to 12 per cent of moisture, a very favorable condition for mold growth. The remedy for this rapid deterioration is simply to dry it so that it contains not more than 5 per cent of moisture, and express the oil as soon as possible, avoiding long storage in a warm, moist atmosphere.

Drying.-By comparing the various methods of copra drying, a hotair apparatus, either rotary or stationary, was found to be the most efficient. It is suggested that a combination of centrifugal with hot-air drying might prove of considerable value, provided a market could be 
obtained for the by-product, "coconut cream." Vacuum drying is not of great value in the desiccation of coconuts for oil-making purposes.

(10) Although a pure coconut oil is not a suitable medium for a growth of micro-organisms, one containing a sufficient amount of nutrient matter and moisture may, under certain conditions, develop a growth of mold which rapidly attacks the oil itself. A sample of pure oil to which had been added 1 per cent of "latic" and 1 per cent of water increased in acidity from 0.10 per cent to 8.63 per cent on standing exposed to mold action in an incubator for one week.

The very slight increase in acidity which a pure oil suffers on long standing is probably due to simple hydrolysis by heat and moisture.

(11) Besides the production of free acid by molds and the decomposition of albumen by bacteria in moist copra and in impure oils, one other factor enters into the deterioration of coconut oil. Many samples on long standing develop a slight but noticeably acrid taste and odor, without any marked increase in acidity. Such oils invariably give a blue coloration with Schiff's aldehyde reagent, reduce silver nitrate in Becchi's test for cotton-seed oil, and possess the power of liberating iodine from potassium iodide. ${ }^{24}$ This process is shown to be a direct oxidation by the air and to depend largely upon the amount of surface exposed. Other conditions favoring it are freedom from moisture and impurities, as is shown by the fact that impure commercial oils, or those which have been acted upon by mold, do not, as a rule, respond to tests for peroxide and aldehyde, while the most marked development of these bodies is noticed in the purest oils.

(12) The action of light and air on coconut oil is of relatively little importance in comparison with the great changes produced by mold growth, and it can be prevented in a large degree by keeping oil receptacles as nearly full as possible, so as to reduce the amount of surface exposed.

${ }^{24}$ Since writing the above I find that L. Legler [Pharm. Centr.-H. (1904), 45, 839] has noticed the same phenomenon in oxidized lard. As a test for active oxygen he proposes to shake the sample with a solution of neutral lead acetate and a few drops of ammonia. A yellow coloration, due to the formation of hydrated lead peroxide, indicates the presence of oxygen. I have applied this test to old coconut oils and find that, with highly oxidized samples, it gives a strong coloration, but it is not as delicate as the simple reaction with potassium iodide. To the presence of active oxygen Legler attributes the reduction of silver nitrate in Becchi's test given by samples of oxidized lard entirely free from cotton-seed oil. I have observed the same fact when applying Becchi's test to pure, but oxidized, samples of coconut oil, but considered it more logical to attribute the reduction to aldehyde-like bodies present in the oil, rather than to the active oxygen. 
THE PRINCIPAL INSECTS INJURIOUS TO THE COCONUT PALM (PART I).

By Charles S. Banks.

(From the Entomological Section, Biological Laboratory, Bureau of Science.)

Among commercially valuable trees, few are attacked by as small a number of insect pests as the coconut (Cocos nucifera L.) ; but, on the other hand, the destructive action of this limited number is very great.

The trunk of the coconut does not have its important conducting tissues in or immediately under the bark, as is true of the cacao, the coffee, or the mango. For this reason, even though the tree were completely girdled, it would not be destroyed, as would be the case with the plants above mentioned. On the other hand, insects attacking the growing point would soon kill this part, after which the remainder would speedily die, and, in fact, this result is the one which almost always is encountered. Certain insects enter the crown and destroy it; shortly afterwards, the leaves turn yellow, the fruits, if any are present, drop off, and the tree eventually dies. It is therefore clear that any method which prevents attacks of this kind will preserve the life of the tree.

This paper will treat of some of the most important of the insects destructive to the coconut which have been identified, while those the habits and life histories of which are known but the determination of which has not yet been made will be a subject for further study. The observations upon the habits and life histories have been made both in the laboratory and in the field. I wish to take this opportunity of thanking Mr. W. Schultze for his hearty coöperation in this work and for the illustrations which he has furnished.

\section{THE RHINOCEROS BEETLE.}

Oryctes Rhinoceros L. (Tagalog, Uang).

This insect belongs to the family Dynastidæ, or that of the giant beetles, a group in which, from the standpoint of body weight, the largest of the beetle tribe are found. This beetle is very common throughout the Philippine Archipelago and in other countries of the East (Ceylon, Java, India, etc.) wherever the coconut tree is encountered. All of the 
species of the genus Oryctes apparently have the same predilection for the coconut and for similar palms. The presence of the rhinoceros beetle is indicated by the large, irregular holes in the trunks of the trees or at the bases of the largest petioles of the leaves of the coconut. These are made by the adult beetles and serve as a means of entrance for other insect pests, such as the Asiatic palm weevil, and also for the admission of moisture, which eventually causes the trunk to rot. The beetles' attacks are confined to the soft tissues near the top of the tree, and holes seen in the trunk below this point date from the time when the growing apex was here located.

No Oryctes has ever been found gnawing the hard, old wood of the trunk of the coconut; occasionally adult beetles are found in these old holes, which, however, are used only as a hiding place during the day. In some of them old cocoons which were constructed when the hole was at the crown of the tree are occasionally found, consisting of masses or bundles of fiber. These have been preserved in situ, because of the small size of the opening of the burrow. As time goes on, the old holes become enlarged through various agencies, particularly through erosion and decay caused by the entrance of water, so that these bundles of fiber finally become exposed.

Life history and habits.-Like all other members of the family Dynastidæ, Oryctes is a vegetable feeder. While it frequently occurs in heaps of decaying vegetation, the larvæ appear to have the greatest liking for the soft, growing point of the coconut, which is the location from which new leaves, the flowers, and, subsequently, the fruit, obtain their nourishment. Therefore, any injury to this part of the tree immediately results in debilitating the whole plant and eventually in its death. The mode of attack most generally encountered is that in which the female has entered between the long stems or petioles of the outside leaves and those immediately subjacent, and then has eaten a hole into the outer side of the inner petioles, which are protected from the light by the external leaf stems. As this beetle shuns the light, its attacks always begin during the night, and by the following morning it will frequently have entered so far into the burrow as to be protected from the light. It then continues its feeding until a gallery of considerable size has been excavated. The habit of burrowing would seem to be not solely for the purpose of laying eggs but also in order to obtain nourishment, as nearly all of the members of this family feed in the adult stage.

The egg.-I know of no record regarding the actual deposition of the egg, nor have I found it in any of the burrows from which the adult has been taken, but, by dissection of the females, the eggs have been obtained in considerable numbers. Just before being laid they are of a dark cream color and present a perfectly smooth texture. The microscope reveals a very delicate reticulation or punctuation of the surface. They are 3.5 millimeters long and 2 millimeters in diameter, being of a perfect 
broad, ellipsoidal form. 'The structure of the ovipositor of the beetle leads one to infer that the egg is simply dropped by the female at any spot in the gallery, where it adheres to the side of the latter until the larvæ are hatched. In none of the females which I dissected were there more than seven or eight eggs of such a size as to indicate that they were about to be laid, so that the insect probably deposits not more than two dozen during its life. (Pl. I, fig. 1.)

The larva.-Length over dorsun when full grown, 112 millimeters; circumference, 18 to 20 millimeters; head, 12 millineters long and 11 millimeters wide; feet, 9 millimeters in length. It is a very soft, fleshy grub, the skin of which is transversely doubled in numerous folds, so that it is very difficult to differentiate the body segments. The skin is of a dirty, light ocher, the surface being smooth in spots and in other parts covered with patches of very minute tubercles or spines, which give it the appearance of shagreen. The body is covered with numerous, very fine, golden or dull-whitish, curved hairs, which stand out nearly at right angles to the surface. The head is of a much darker color than the body and horny or chitinous in structure; it is hemispherical and so attached to the body that the mouth projects forward. At each side of the head, projecting downward and forward, is a slender, four-jointed antenna. The dark-brown, slender, toothed mandibles, half the length of the entire head, are so placed as to enable the insect to gnaw its way through the plant substance with great facility. The maxillæ are rather conspicuous and are situated next to the mandibles on the under side of the head and on each side of the very inconspicuous lower lip or labium. The maxillary palpi are 4-jointed; those of the labrum 2jointed. The labrum is transversely elliptical, the sides slightly covering the inner margins of the mandibles. The color of the labrum and that of the clypeus (the trapezoidal portion above the upper lip) is the same as that of the rest of the head. The mouth parts, with the exception of the tips of the mandibles, are covered with golden bristle-like hairs, which serve as tactile sense organs.

This grub has no eyes. The top and front of the head, therefore, present an unbroken surface, which is somewhat shiny and covered with punctures, which are almost confluent; it is nearly destitute of hairs or bristles. (Pl. I, fig. 2.)

Each of the first 3 segments of the body posterior to the head bears a pair of legs. The first leg joint projects downward, while the succeeding ones are inclined outward and forward; the feet are armed with a single blunt claw and densely covered with light-brown bristle-like hairs, more thickly placed at the tips.

The body is curved, so that the length of the ventrum is much less than that of the dorsum. It is folded or transversely corrugated so as to render it difficult to distinguish the 13 segments of which it is composed, this being the easier toward the anal extremity, where the folds are fewer.

Beginning with the first thoracic segment and excepting the second and third, each remaining one to the eleventh bears a pair of dark-brown subcircular spiracles or openings to the tracheal or respiratory system. These spiracles are chitinous in structure and are composed of an outer broken ring of radiating lines and an inner nearly circular portion, which is the true opening. They may be opened or closed at will. Their large size and great prominence is doubtless owing to the fact that the insect lives embedded in a mass of material in which the supply of oxygen is limited.

The last three segments of the grub's body are nearly smooth and only sparsely covered with hair, and in a living specimen the hinder end is semitransparent 
and contains a dark mass of material, consisting of the partly digested cellulose fibers of the plant upon which the insect has fed. The anal opening occurs as a transverse slit at the extremity of the body. (Pl. IV, fig. 1.)

Differences of opinion appear to have existed with reference to the destructiveness of the grub of Oryctes. Blandford says:

They are harmless, and live in heaps of rotting vegetable matter or the manure-like inside of decayed paln trees.

Both Mr. Schultze and I have discovered them in large numbers in coconut trees in which the "manure-like" material inside the trunks gave every evidence of having been made by the grubs themselves. One tree, felled in the town of Magdalena, Laguna Province, while still alive and to casual observation fairly healthy, was found to have an inverted cone eaten out at the crown, as shown by Plate III, fig. 1. This contained seven of the grubs of Oryctes rhinoceros L. buried in the frass. There was a tunnel, 3 centimeters in diameter, extending down from the apex of the cone for a distance of 90 centimeters through the heart of the tree, and at the bottom of this tunnel was a full-grown grub, which to all appearances had eaten its way to this point. Mr. Schultze observed in Pagsanjan a tree (Pl. II) 5 meters high, the whole of the interior of which had been eaten out from its top to within a half meter of the ground, leaving a shell with a wall from 15 to 20 centimeters in thickness. Within this, at the lower part, was a mixture of water and decayed matter 50 centimeters deep, indicating that the work of Oryctes and the Asiatic palm weevil, together with infiltration from the top, had been continuing for a considerable period of time. Within this rotting mass and at intervals up to the crown of the tree were found the fiber cocoons of $O$. rhinoceros L., while from 75 to 100 larvæ of all sizes, from 5 millimeters to the full-grown grub, were removed. The small number of weevils, the large number of Oryctes larvæ and pupæ, and the general appearance of the interior of the tree furnished conclusive proofs that the work was that of the insect in question. Leaving these points aside and reasoning from the anatomy of the larva alone, it is evident that it could work in the wood of coconut with great ease, since it is in every way fitted for burrowing there. If it lives only in manure heaps or in decaying matter, it would appear that there would be no necessity for such well-developed and powerful mandibles, nor would the head have to be of such hard material. It is true that these grubs are always encountered in the presence of decayed matter, either in the tree or in manure and other vegetable heaps, but, when found in the tree, it is probable that the decaying material is a result and not the cause of their presence. It is also true that we never cut into a tree until it shows unmistakable signs of insect attack or disease, and therefore do not see the work of the beetle at its incipiency. I have seen larvæ one-quarter grown removed from the burrows made by the adults in small coconut trees, the leaves of which were pulled apart; and I have also observed the 
removal of grubs with a piece of bent wire from young coconut trees, although at the time I did not examine the hole sufficiently to note the actual work of the larvæ.

However, it is not to be doubted that these same insects find a suitable place in heaps of decaying vegetable matter, as the grubs have been found in such locations in all stages of growth. In connection with this question, Father Stanton, formerly of the Manila Observatory, ${ }^{1}$ makes the following observations:

We have fourd several live pupæ in a partly decayed stump of Pithecolobium saman that had been lying on an old wood pile for months; at another time we discovered one in a neat oval earthen cell within a broken bottle lying in a heap of refuse near a stable; and on one occasion, in a single heap of earth and manure, within a space of 1 cubic yard, we gathered dozens of larvæ in all stages of development from specimens 1 centimeter in length to those of 12 centimeters just about to transform to pupæ together with half a dozen pupæ and as many perfect beetles with their elytra still rather soft, as though the insects had just emerged from the pupal envelope. In this latter case, at least, it appears quite evident that the whole cycle of the metamorphoses of the insect took place right in this small pile of manure or very near to it. For, as many of the larvæ were very young, they couId not well have migrated from the interior of a coconut or buri palm, seeing that there was not a single one of these trees in the whole neighborhood. It is evident then that 0 . rhinoceros does sometimes pass its whole larval and pupal existence in the midst of decayed or dęcaying organic matter, and consequently that the eggs are deposited in such situations. Whether they are also laid in the holes made by the female in the living tree is still to be ascertained, though from the fact that the grubs are sometimes found feeding in the heart of the tree high up near the crown it seems quite probable.

He quotes Señor Vicente Reyes, of Santa Cruz, Laguna, who says:

It is remarked that coconut trees with all the leaves fresh, with blossoms and fruit all in perfect condition, and without any apparent cause, fall to the ground as though a hurricane had cut them down. On being examined it is found that from the roots up to the distance of a meter above the surface they are completely hollowed out, the whole interior having been converted into a mass of sawdust, and ensconced therein are a number of these worms, which have entered from the roots and worked upward, little by little, eating away and living upon the substance of the trunk itself.

In every case where I have encountered Oryctes in trees, except in those which were completely hollow, the work evidently proceeded from above downward. Of course, in those which were hollow, the channels of the grubs were found along the inner surface of the shell of the tree, but the evidence thus exhibited was not conclusive as to whether the larvæ had worked from above downward or the reverse.

Father Stanton notes the finding of the larvæ, pupæ, and adults of Oryctes in manure and other decaying organic matter, but he also says that he has not ascertained whether the eggs are laid in the holes made by

${ }^{1}$ Phil. Weather Bur., Bull., August (1903), 225. 
the adult beetles in the trees, adding that the fact that the grubs are sometimes found feeding in the heart of the tree high up near the crown makes it seem quite probable that the eggs are laid there also. Every evidence in my experience points to the great likelihood that they are laid in these holes.

When a tree which is so badly infested as to give external signs of debility is cut down, one usually finds larvæe of all stages as well as pupær. The question of the length of the life period of this insect is a difficult one to determine, but from examinations, such as it has been possible to make during the time the insects of coconut have been under special observation, I am led to believe that it varies from eighteen months to two years, according to the food conditions. 'These conditions are determined largely by the size of the plant and the proportional number of insects in it.

Pupa.-The pupa of a female measures about 50 millimeters in length and 25 millimeters in width. The distance over the back from the tip of the head to the hinder part of the body, which in the pupa is curved forward, is 65 millimeters. It is of a light ocher yellow, in certain lights presenting a bright satin sheen and in others a velvety appearance. The head, thorax, abdominal segments above and below, and the wings and legs are all plainly visible, the anterior apex of the pupa, at a point corresponding with the top of the head in the adult insect, shows a small sharp knob or tubercle, which represents the horn of the full-grown beetle. A very fine golden pubescence, covering certain areas of the pupal body, causes its velvety appearance. The spiracles are placed similarly to those of the larva, but are almost hidden by the folds or wrinkles of the abdominal segments. On each side of the middle line of the back of the abdomen, transverse slits, very much like spiracles in appearance and undoubtedly secondary breathing orifices, are seen. These occur between each two abdominal segments, beginning with the first and continuing to the seventh, inclusive. Between the seventh and eighth there is indication of their existence in an atrophied state. (PI. IV, fig. 2.)

Cocoon.-The cocoon is composed of fibers of the coconut, wound transversely and rather compactly woven or matted together. It sometimes measures 100 millimeters in length and 40 millimeters in diameter. When these insects live in rotting material or manure, the cocoon consists simply of an oval excavation, the interior being smoothed by the larva previous to its transformation. Unlike many pupæ of insects which feed in the interior of masses of material, this one has no organs by means of which it may cut or push its way out of the cocoon at the moment of transformation to the adult.

Adult.-The full-grown insect varies in length from 34 to 48 millimeters, according to the sex and the amount of nourishment taken in the larval stage, the average for the males being 44.2 millimeters and that for the females 37 millimeters. They are of a very dark-brown, somewhat lighter beneath, and have a very glossy or shiny appearance. The most striking feature is the horn on the fore part of the top of the head, this being much larger in the male than in the female. The head, thorax, and abdomen are easily distinguished. (Pl. IV, fig. 3.)

Male.-The head, with the exception of the horn, is irregular in form and subglobose; the front is strongly emarginate or sulcate. It is small in comparison with the thorax and so concealed posteriorly by the thorax, into which it fits very snugly, that it appears to be subtriangular from above. The eyes 
are black and shiny and so situated at the sides of the head as to be half concealed by the anterior margin of the thorax. The anterior margin of the orbit is extended so as almost to cut the eye into upper and lower sections. The portion of the head at the base of the horn. which extends upward directly from the clypeus, is very densely pilose or setose, as is also the frontal sulcus. The occiput has an emargination at cach side of the median line; into these fit the strong tendons of the powerful muscles which move the head upward. The antennæ project from the under, outer margin of the head. They are composed of 11 segments, the apical 3 of which are laminate; the first is swollen at the apex, is as long as the succeding 7 together, and is very strongly pilose, the bristles being on its anterior, external surface (Pl. I, fig. 3). The ninth and eleventh segments are also pilose at their outer margins and tips; the tenth, lying concealed between them, is smooth and blade-like. The small, 4-jointed maxillary palpi lie just beneath the insertion of the antennæ at each side of the labium, which is subquadrate, with the anterior surface strongly swollen. Its lateral margins are strongly setose. The maxillæ are laminate and hidden between the labium and the peculiarly shaped mandibles. They are strongly setose on their exterior margins. The 3-jointed labial palpi lie beneath or anterior to the maxillary palpi and are attached to the apical part of the lateral margin of the labium.

The most peculiar feature of these insects, which has hitherto been unmentioned in the literature on the habits of the adult, is the special form and function of the mandibles. In the general description of the genus to which this insect belongs, the statement is made that "the mandibles are prominent and sometimes toothed externally." In the rhinoceros beetle the external tooth of the mandible is curved upward and forward and has the form of the cutting edge of a nonconcave gouge. These teeth, one on each side of the head, are by their construction and that of the surrounding parts well adapted for chiseling out the wood of the tree. (Pl. I, fig. 4.)

The shape and position of the external mandibular teeth, the form of the mentum, or chin, which is rounded and curved vertically, and which fits into a groove having a like form, in the anterior margin of the prothorax, together with the strong, well-attached muscles at the back of the head, prove conclusively that the insect, instead of gnawing its way into the tree, chisels into it by an upand-down motion of the head, and it is my belief, for reasons to be given later, that no part of the wood is taken into the body.

The horn of the male is 10 millimeters in length and 4 millimeters in width at its base, tapering gradually to 1 millimeter at the tip, which in many specimens appears as if worn off and repolished. It is sparsely punctured, these punctures being fewer toward the tip.

Thorax.-The pronotum occupies about one-third of the length of the insect on the dorsum, is roughly subcircular in general outline, narrower anteriorly, having the margins somewhat reflexed. The anterior two-thirds shows a large, shallow depression, the surface of which is transversely or concentrically striopunctate, and at the posterior margin of which are two rather inconspicuous tubercles, almost coalescing. On each side and in the forward angle of the pronotum there is an irregular depression, posterior to which and extending narrowly around the posterior margin of the main depression, is another parenthesis-shaped one, broader anteriorly and having its surface roughly rugose. A line of submarginal punctures extends around the pronotum. (Pl. I, figs. 5 and 6.)

Elytra.-At the base, the wing covers are as wide as the thorax, the surface at the outer basal angles being quite smooth. On each elytron are four lines, one of which is subsutural, extending from base to apex. The external ones are 
very indistinct toward the apex. The part between these lines is coarsely punctured, the punctures being regular on each side of the three external lines and on the exterior of the subsutural ones. The triangular scutellum, between the bases of the elytra, is smooth at its apex. There is a triangular rugose or coarsely irrorated area at its base.

The under surface of the thorax is of a chestnut brown; it is highly polished on the areas against which the legs move and strongly punctured on other exposed parts, the punctures having a sparse pile of light-brown bristles.

Legs.-The femora are uniform in size and smooth, each with 1 row of setose punctures nearer the posterior ventral margin. The tibiæ are nearly similar in shape and size, bearing externally 3 rather prominent teeth. The fore tibiæ, in addition, have an internal and external apical one. The mid and hind tibiæ have each 1 internal and 2 external apical teeth, armed with a row of smaller secondary ones, and all are coarsely punctured. All the tarsi are of about the same shape and size, except that the last joints of the anterior ones are slightly longer than the others, and the first of the mid and hind tarsi are subconical and slightly larger than the succeeding ones. All tarsal joints are setose or spinose at their apices.

Abdomen.-The dorsal abdominal segments are hidden by the elytra; the 6 visible ventral ones are smooth, except for very sparse punctures and a subapical row of setose punctures on each. There is a tuft of brown hairs at the anal slit. The hinder exposed part of the abdomen is rounded, smooth, shiny, and sparsely punctured. The elytra do not cover the last 2 dorsal segments.

The principal differences between the female and the male are that the former is much smaller and its horn may be a mere tubercle, or, at best, not more than one-fourth as long as that of the male. The depression on the pronotum extends back less than halfway; the posterior lateral rugose areas are somewhat broader. (Pl. I, figs. 5, 6). The last ventral abdominal segment of the female differs from that of the male, in that in the former it is rounded and covered with bristly hairs, while in the latter it is markedly emarginate. The ventrum of the abdomen of the female is rather densely covered in transverse rows with bristles, except along the apical margins of the segments. The posterior part of the pygidium is also densely hairy. Both sexes have on all the thoracic joints, as well as at the articulation of the head with the thorax, a fringe of bristles closely applied to the surface upon which the part is attached to prevent the entrance of foreign matter between the sutures.

Method of operation of the adult.- The method of attack of the adult insect was formerly believed to consist in its gnawing into the plant for the purpose of feeding upon the soft tissues inside, its eggs not being laid in the tree. This view is partly incorrect. Observation has shown that the males make burrows, as well as the females, and it is probable that they always accompany the latter at the time of egg-laying, retreating from the burrow they have made to allow the female access. Dissection demonstrates that the stomach of the insect contains no masticated fiber; on the contrary, it is filled with a dark, amber-colored liquid; nor are there any fiber cells found in the excreta. The proventriculus or gizzard of the insect is not provided with walls for grinding and the mandibles are constructed somewhat like the parts of a cane mill through which the sugar cane passes in expressing the juice, except that their surface is corrugated, the elevations of the one fitting into the depressions of the other. 
The œsophagus is not more than 1 millimeter in diameter. The insect begins the process of separating the fibers of the tree by means of its chisel-like teeth. The rapidity with which a beetle can work is shown by the fact that within half an hour it will have entered a fourth of its own length into the plant tissue, and when once it is enabled to brace its strong-spined legs against the walls of the burrow its progress is accelerated. The heart of the tree is its objective point.

On Plate $\mathrm{V}$ are shown successive layers of leaf petioles at the heart with the burrow of an adult which finally reached the center. Figs. 1, 2, 3, and 4, respectively, show the pieces from outside, inward.

An examination of the fibers as soon as they are cut by the bectle demonstrates them to be almost dry, which renders it more probable that the purpose for which they are taken into the mandibles is solely to extract their juice, after which they are expelled from the mouth. Plate V, fig. 4, shows the heart of the coconut tree with a beetle at work. The bits of tissue which have been chiseled off can plainly be seen. In less than ten minutes the insect had burrowed into the soft substance for a distance of 10 millimeters. Plate III, fig. 2, shows the initial work of a beetle in a leaf petiole.

The beetles fly only at night; in the daytime they are readily found in their burrows. Their wings are quite large and the wing muscles in the thorax are strong and adapted for the flight of such heavy, unwieldy insects. In the interspaces between the intestines and the reproductive organs the abdomen is filled with air sacs and tracheæ.

Extent and character of damage done.-It is rare to find a single coconut tree anywhere in the Philippines which does not show one or more evidences of attack by this beetle. It is the pest most frequently reported by farmers and coconut growers, and in hundreds of trees which I have personally examined large holes in the trunk, distorted leaf stems, or ragged leaves demonstrate the character of its work. The insect larva or the adult, in its work inside the tree, frequently cuts off the tip of the embryo leaf or the tips of the leaflets on one or both sides of the midrib, so that when the leaf finally grows it appears as if it had been trimmed with a pair of shears or as if a triangle had been cut from one or both sides. The fibers severed by the insect protrude from its burrow, giving the latter a ragged appearance. During the daytime the beetles are frequently encountered in very old holes, into which they evidently have gone for the purpose of hiding. They have never been seen further to excavate these old cavities. The openings which are made serve to allow rain water to enter the tree, where it causes a most rapid decay of the interior, and they also serve as an entrance for other insects quite as destructive as the coconut beetle.

Distribution.-Oryctes rhinoceros L. is probably tropicopolitan, being found in Honduras, India, Ceylon, Java, the Philippines, Celebes, Borneo 
and Sumatra, and recorded as coming from Africa. It undoubtedly thrives well wherever the coconut is grown.

Dr. Köningsberger, of Buitenzorg, Java, says: "The well-known coconut beetle $O$. rhinoceros $\mathrm{L}$. is one of the most terrible enemies of coconut culture." And if this be the case in Java, where cultural methods have been in vogue for so many years, it is probably much truer in the Philippines.

Preventive and remedial measures.-The question of controlling the ravages of this insect is a difficult one. It would appear that trees growing to such a height as the coconut and having so few parts would not be seriously affected by a rank growth of weeds or underbrush or by a lack of cleanliness in their surroundings, but this is certainly not the case. It has already been stated that the larvæ of the coconut beetle grow in manure and rotting vegetable heaps and also thrive in rotten or rotting coconut trees, their abundance appearing to be in direct ratio to the degree of decay which the tree has attained. Mr. Schultze has taken as many as a hundred larvæ of all sizes from the decayed shell of a coconut trunk. I have invariably found them in great abundance in such situations. It is obvious that these sources of infection for healthy trees must be removed. The first thing to do in coming into possession of a coconut grove or in planting a new one is thoroughly to clean the ground. All manure heaps, rubbish, rotting or fallen trees should be removed and destroyed at once. 'The manure should be scattered where it will serve the best purpose as a fertilizer, and in such a manner as to make it impossible for the grubs to find lodgment in it. Rubbish heaps and decayed trunks, if fallen, should be burned; if standing, should be cut down and burned. The residue can easily be returned to the soil as fertilizer.

Growers should not remove the dead leaves from their trees to such an extent as to leave the young and still tender petioles or leaf stems entirely exposed, thus inviting attack by the adult beetles. These leaf stems have a thorough natural protection by being wrapped in a woven fiber, the old stems remaining upon the tree until the new ones are fully grown. When the living leaf stems are cut off a foot or two from their union with the trunk, the sap running out offers an attraction to beetles which might otherwise not attack the tree. Blanford discusses this danger as follows: ${ }^{2}$

"The trees should be left, as far as possible, in the natural state, and unnecessary trimming either of fronds or of the fiber avoided. It may be necessary to tie up the older fronds, and, if they must be removed, the stalk should be cut through sufficiently far from the stem to leave the sheathing base intact. It may be advisable to tar the cut stump if it is found to attract beetles. The value of leaving the trees alone is shown by a passage in Ferguson's All about the Coco-nut Palm, which is also quoted by Ridley: 'Scores of instances might be recorded

${ }^{2}$ Kew Bulletin (1893), 73, 46. 
where, till the trees were come into bearing, a red beetle was never seen, but no sooner was the land cleared and the trees trinmed than it made its appearance and became very destructive. On one property the trimming system had been carried on for years, till, indeed, more than one-third of the original plants perished, before the estate was 10 years old, and they were going at the rate of three trees weekly. The work of trimming was stopped for the reasons offered above; the loss of the trees continued for some time afterwards, but at the end of six months it had entirely ceased. On another property beetle men had been employed for ten years, and trees were being constantly lost; from the day that the beetlers were discontinued two trees perished within a month, and not another was lost in the subsequent seven years.' And W. B. L. writes in the Tropical Agrioulturist to the same effect: 'The red beetle (Rhynchophorus ferrugineus) can not penetrate the leaf imbrication, and, when the older ones decay in the course of nature, the stem has become too hard for its operations. A tree here and there may be lost from an accidental wound or from some defect in the fitting of the leaf sheaths, but it is only where the good taste of the planter has impelled him to trim the leaves that any serious damage has been done to a field. All the leaves should be left on the tree till nature disposes of them at her own time and in her own way. Nothing that can be done to a coconut tree above ground can be anything but injurious.'

"All wounds, whether made by accident or by insects, on the soft part of the stem, leaf sheaths, or spike should be at once dressed with a dab of tar mixed with fine sand. Holes should be probed with a "beetle spear" or hooked wire to extract insects which may have caused them and then plugged with a tuft of fiber or dry grass dipped in tar."

Obviously, no tree should be condemned until a careful and thorough inspection makes it certain that it is beyond hope of recovery and that it can bear no more fruit. It has been suggested by certain writers that a good plan is to cut such felled trees open, leaving them on the ground to attract beetles which would otherwise fly to the healthy trees; but I am of the opinion that the less material of this kind there is in the orchard the less is the liability of attack incurred by the bearing individuals. If there are no wounds or vulnerable spots in the trees themselves, and if nothing remains on the premises to attract this beetle and others, the less will be the danger. The dead leaves should be allowed to fall in the natural course of growth and care should be taken not to mutilate the trees. However, in most instances the beetles already have invaded the plantations and the serious problem is how to rid these places of them and to prevent their reëntrance. Of course, frequent inspections are necessary, so as to detect invasions at the earliest possible moment, because, as the coconut beetles hide in their burrows during the day, it is comparatively easy to destroy them if they are noticed in time. When they are discovered, a long, hooked steel wire can be thrust into the burrow, given a half turn to engage the insect upon its point, and then drawn out. This operation requires some practice, as the beetle is well armored with a smooth coat and has few projections upon the body. Dr. Könings- . berger suggests crushing the insect and leaving it in the burrow as an obstacle to the entrance of others; but this is not to be recommended in 
the Philippines, because the dead material would be sure to attract ants, which in turn would draw other insects, such as white ants (anay) and serious complications might arise. When the insect has been killed and removed, it is essential to plug the hole with some substance which will prevent further attacks at that point. For this purpose various substances have been recommended, for example: tar and fine sand, plaster and sand, clay and tar, or, in place of clay, plaster or cement. This mixture should be forced into the holes as far as possible, because it then will act as a deterrent to the decay caused by the entrance of moisture subsequent to attacks of the beetle, while effectually closing an avenue of entrance for others. Another remedy is to use a paste of Paris green and flour, mixed with 10 or 12 gallons of water, and sprayed into the crown of the tree. This method would offer difficulties when tall trees are to be dealt with, owing to the impracticability of getting the spray to the right places. The Filipinos use various remedies, such as sand and coarse salt, which they place in the crown of the tree. They state that the sand gets between the articulations of the head and thorax of the beetle, where the constant friction sets up an irritation which eventually punctures the soft tissues, after which the insect dies. This may be true. There is a constant movement of the head and of the thorax, while the beetle is working its way into the tree, and although the articulations are protected, as explained above, by a fringe of closely fitting bristles, it is possible that fine sand might enter as suggested and thus seriously handicap the beetle in its boring operations, if not eventually killing it.

I have been assured by a gentleman who is one of the most successful farmers in the Islands that natives on his plantation pour urine into the crown of the affected coconut trees and that this method effectually rids them of the pest. This is certainly not impossible.

\section{ASIATIC PALM WEEVIL.}

\section{Rhynchophorus ferrugineus Fabr.}

"It has been observed that coconut palms, the green leaves, blossoms, and fruits of which appear in perfect condition, fall to the ground, without having any signs of decay, as though struck by a hurricane. In such instances it has been noted that (the trees) from the roots up to a meter in height, are completely undermined, the interior pulverized like sawdust and filled with nests of these worms, which have gained entrance through the roots and gnawed their way upward, deriving maintenance from the trunk." 3

The gravity of the attacks of the Asiatic palm weevil is well summed up in the foregoing extract, for, while the condition referred to is not generally reported from all parts of the Islands, there is every reason to

${ }^{8}$ Extract of translation of a communication from Señor Vicente P. Reyes, of Santa Cruz, Laguna, with reference to $R$. ferrugineus Fabr. 
believe that in general the depredations of the beetle are no less serious in their ultimate effects than in the cases reported from the Provinces of I aguna and Tayabas, in which regions Señor Reyes has seen the damage to which he has made reference. I visited Magdalena, Province of Laguna, which lies in a fairly productive coconut region, and have found conditions closely resembling those set forth above, except that only a very few trees has actually fallen. In most instances where this had occurred the stumps had been cut off rather close to the ground during the previous year, and hence we found little material at hand upon which to work. However, we were convinced that the menace to coconut growing from this insect is fully as serious as, if not more so than, that occasioned by the attacks of Oryctes rhinoceros $\mathrm{L}$.

This weevil enters the tree through the smallest wounds, leaving no external trace of its work, so that all its ravages are committed where not suspected; hence it is an extremely difficult enemy to combat.

The Asiatic palm weevil belongs to a group the members of which are, almost without exception, destructive to vegetable substances, either living or in the form of stored products, such as grains, beans, pease, and nuts. The beetle under discussion is one of the largest of its kind. The rice weevil is not more than 5 to 6 millimeters long; the corn weevil, Calandra oryza Linn., is slightly larger; the boll weevil, Anthonomus grandis Boh., which is at present proving so serious a menace to cotton growing in the United States, measures about 5 millimeters in length; the plum curculio, Conotrachelus nenuphar Hbst., a weevil, is 6 millimeters long; while there is another species attacking the coconut which measures 13 millimeters. The Asiatic palm weevil measures 35 millimeters in length. The form is strikingly characteristic in all individuals of this group. The most prominent features are an oblong, oval-shaped body and a long, slender, curved snout or bill, to which are attached the antennæ, either near the base or the tip. The colors vary from black to light brown or red, but are usually obscure.

The larvæ are legless, with a head of chitinous or horny structure, usually darker than the body and having two strong mandibles well adapted to gnawing the hardest vegetable substances. The Asiatic palm weevil has never been seen to make a primary attack upon the hard wood of the coconut; wherever it has been observed, it has utilized the holes made by Oryctes, wounds carelessly made around the base of the tree, or the steps cut into the sides of it by the tuba gatherers. Wherever the hard bark is broken and the softer parts beneath exposed, excellent places for the laying of the eggs are found and the beetle often makes a hole 10 millimeters deep before depositing thern. The character of the hole and the tracks of the larvæ after hatching are shown diagrammatically in Plate VI, fig. 4. In laying their eggs in the burrows made by Oryctes, the palm weevils undoubtedly make no appreciable hole, simply 
forcing the egg a short distance into the soft material in which the burrow lies.

Egg.-The egg is slender, 2.4 millimeters long and 0.6 millimeter wide at the middle, slightly more pointed at one end than at the other, and of a very light ocher. The shell appears perfectly smooth and shiny, but when examined under the microscope the surface is seen to be finely reticulated. (Pl. VI, figs. 1 and $1 \mathrm{~b}$.)

Larva.-The larva does not vary essentially in general characteristics from the time of hatching until it is fully developed. Full grown, it measures from 35 to 55 millimeters in length and from 18 to 22 millimeters in diameter. The greatest diameter is slightly behind the middle. The hinder part of the body forms a concavo-convex extension, a blunt spoon or scoop-shaped organ.

The head is from 10 to 12 millimeters long and 7.5 to 8 millimeters wide. Seen from above, it is of a regular oval outline. It is of a dark-chestnut brown, with a slight reddish tinge, and with a lighter median and 2 submedian narrow stripes marking the sutures. (Pl. VI, fig. 2.)

The space around the mouth parts and the latter themselves are of a dark brown, with the exception of the upper lip and clypeus, which are lighter. The triangular portion of the head, immediately above the mouth, is transversely rugose, with a longitudinal furrow on each side of its middle. The remainder, including the cheeks and occiput, is engraved with very shallow reticulations, giving the appearance of a piece of alligator skin in miniature. The smooth, dark-brown, subtriangular, rather blunt mandibles are exposed on each side of the mouth; the upper lip, or labrum, lying between them, and reaching nearly to their tips, is provided with numerous bristle-like hairs. The larva has no antennæ, but the maxillary and labial palpi are well developed and doubtless serve as feelers. It has no eyes. The underlip, or labium, is subtriangular and rather small, but quite fleshy; the palpi project conspicuously from each side of its tip. (Pl. VI, fig. 3.) lt is supposed that the surface of all these organs is highly sensitive and that the insect can tell desirable food by touch. The head shows erect hairs placed at regular intervals, 5 on each side of the top and 3 back of each mandible. Portions of the front of the head, and the mandibles, appear as if having been rubbed off by friction with the substance in which the larva lives, so that these parts have a dull, almost black or matte appearance.

The body is composed of a series of 13 rings very much folded and wrinkled, the surface being of a smooth, velvety texture, except in certain spots, which are decidedly shiny. On the back of the first segment appear 2 transverse, oblong patches of a darker color than the remainder of the body, with a surface similar to that of the head and serving as a protection to the animal in its movements in the small galleries in which it works. There are similar lighter areas at the sides of the first 3, or thoracic, segments, which are somewhat swollen and serve in lieu of legs. Scattered over the entire surface are tiny, circular or irregular shiny areas, froni each of which arises a small curved bristle. On next to the last segment of the body, dorsally situated, are also irregular shiny patches, each with 6 bristles. The last segment has the upper surface concave, and the lower convex; the posterior margin, which is slightly darker than the rest and smooth and shiny, is flattened out and has four prominences, from each of which project two rather long bristles. The spots from which they project are lighter in color. The wrinkles of the body are nearly symmetrical. (Pl. VII, fig. 1.) The body curves downward, so that the back is very convex, while the underside is somewhat concave, except just back of the middle, where it is convex, then suddenly tapering to the tip. 
'This grub works its way forward through its burrows by a combination of twisting and undulating motions. In this it is aided by the tubercular enlargements on the thoracie segments. 'The hind end appears to offer no help in this respect. It can enter from any opening through which the head will pass. 'The bristles on the head serve as guides for the insect in passing through holes. When placed upon a level surface, the grub moves slightly sidewise, almost always upon its side, and can thus make fairly rapid progress.

The breathing apparatus in $R$. ferrugineus Fabr. consists of only two pairs of spiracles which are well developed, the others being almost rudimentary. Each of the first pair is situated laterally on the first thoracic segment, and twice its own length below the shiny, transverse, shield-like areas, and the second pair just above the spoon-shaped excavation of the thirteenth segment of the body. The latter two are one and one-lialf times as large as the first two, and their openings are nearly vertical, diverging slightly below. The other segments of the body show the spiracles only when examined under a strong lens; these are nonfunctional, or at most only very slightly so.

The galleries of this grub run obliquely through the large swollen part of the tree near the roots. (Pl. VIII, fig. 3.) 'The specimen here depicted was full of grubs of all sizes and contained one or two pupæ as well. Adult beetles were also found in considerable numbers. The grubs have been encountered in the crown of the tree in numbers varying from 15 to 20 , where they work side by side with those of the rhinoceros beetle, and it is very difficult to distinguish the galleries of the one from those of the other. Plate III, fig. 1, shows a longitudinal section of the crown of a tree which has been eaten out in the form of an inverted cone by the larvæ of the rhinoceros beetle and the palm weevil in company. Plate VIII, fig. 3, shows the work of the weevil in the lower part of the tree very near the roots, some of which are seen at the lower right-hand corner. It will be noted that the galleries run obliquely, which shows that the grubs work inward and upward from the outside of the tree. In this case the eggs were evidently laid in wounds in the root region, on the left, and the grubs worked their way toward the center where the full-sized galleries are seen.

Pupa.-When the larra of the palm weevil has attained its full size, it ceases feeding and evacuates the alimentary canal, thus causing a shrinkage of one-third in its size immediately before making its cocoon. This is elliptical in outline, from 8 to 12 centimeters long and 5 to 6 millimeters in diameter, and composed of the long tough fibers of the coconut trunk wound as shown by Plate VII, fig. 3. It is closely woven and thick, so that the pupa is well protected against dampness. The grub sheds its skin and takes the form shown by Plate VII, fig. 2. The pupa measures 35 to 40 millimeters in length and about 15 millimeters across its widest portion. The snout is doubled down on the breast; the antennæ, wings, and other organs of the beetle are plainly visible. The color is a uniform pale-ocher, the tips of the knees being darker. Rugose areas are situated on each side of the head, back of the eyes, on the upper part of the snout, on the outer fore and hind regions of the pronotum, on the ridges of the elytra, and on the dorsum of the abdomen. These areas are rather thickly 
set with short, sharp spines, which aid the beetle in escaping from the pupal skin by holding the latter firmly in the cocoon. The spiracles, which on most of the abdominal segments in the larva were nearly obsolete, are very prominent in the pupa, a pair on each of the first 6 segments and a fainter one on the seventh being visible.

Up to the present it has been impossible to ascertain the length of time of either the larval or the pupal stage. The beetles begin their work in the trees at practically the same time as Oryctes and the adults are found together with those of the latter, so that the life periods in the larva and pupa of both are probably about the same, or from 18 to 24 months.

Adult.-Rhynchophorus ferrugineus Fabr. is an extremely variable insect, in its markings as well as in its size. Specimens have been obtained of from 25 to 35 millimeters in length, while the color varies from a true ferruginous, with certain black markings more or less regularly placed, to almost entirely black, with only traces of ferruginous. (Pl. VIII, fig. 1.)

\section{Rhynchophorus sp.}

This species is very closely related to $R$. ferrugineus Fabr., if not identical with it, merely varying in general color and in having a broad, ferruginous, longitudinal band from the front to the hinder margin of the thorax. (Pl. VIII, fig. 2.)

The habits and the immature stages of this insect are similar to those of $R$. ferrugineus Fabr. These beetles are found indiscriminately in company on the same tree, and no differences are noted until the adults are compared.

Preventives and remedies.-The prevention of the first attack of the pest is essential. The adult male or female can not bore into the solid tissue as can that of the rhinoceros beetle, because the snout is small and the mandibles are relatively weak. For this reason the female seeks wounds or holes of any kind in the trunk of the tree to deposit her eggs. These wounds may have been caused by other insects, or they may be accidental. One of the chief injuries to the trunk of the tree is that caused by the gatherers of tuba, who make notches in it whereby they may be enabled to climb to the top. As these notches are of considerable size and depth, they offer excellent facilities for the beetles to enter and hide or lay their eggs. All such mutilation of coconut trees should certainly be prevented, even if it be necessary to construct bamboo ladders, securely fastened to the trees, as is done in some localities. There are frequently encountered in coconut plantations trees the bases of which seem to have been chopped with no apparent purpose in view. Of course, these offer an excellent opportunity for the beetles to begin their work. A good, healthy, vigorous, uninjured coconut tree is practically invulnerable to the attacks of the palm weevil.

If, in spite of all precautions, the weevils gain entrance to the tree, the work of combating them is exceedingly difficult. Frequently, when 
they are in the softer, upper parts, it is possible to dig them out with a wire hook similar to the one mentioned as effective against the rhinoceros beetle larvæ. In every case where these or other larvæ are dug out of a burrow, this should at once be filled with a substance distasteful to the adult beetles. Great care is necessary in the work of extracting the larvæ, lest it should be carried to such an extent as to debilitate or kill the tree. If the weevil larvæ are located at or near the base of the tree, where it is almost impossible to dig them out, the only practical method is to stop all avenues of escape and then to remove the tree after it ceases to bear fruit. It has been suggested that infested trees be cut down, split lengthwise, and then left to attract beetles from the others. I am opposed to such a procedure, because it would surely attract fully as many insects from a distance as it would from the immediate neighborhood. Such a method would be advisable only if the other trees were in great danger from beetles already present in them. Plate II shows a coconut tree very badly infested by both the rhinoceros beetle and the Asiatic palm weevil. It will be seen that the entire interior has been eaten out and converted into a mass of débris, in which both the cocoons and the larvæ of these insects were found in great abundance. The tree had cased to bear, the growing point was gone, and there remained only a circle of older leaves, kept alive by the small flow of sap in the outer shell of the trunk. It is obvious that such a tree is a source of infection for a large area.

THE SHOT-HOLE COCONUT WEEVIL.

This destructive weevil has been found in Laguna Province in considerable numbers. I once felled a dead coconut tree, the trunk of which was completely pitted from top to bottom by the insects' exit holes, and Mr. Schultze found the larvæ and pupæ as well as the adults in a living tree. (Pl. X, figs. 1 to 5.)

$E g g$.- The egg has not been found. It is probably laid directly in the hard wood in small cavities made by the female, as the grub can work in any part of the trunk of a tree.

Larva.-The larva is a very pale-yellow, almost white grub, measuring 16.5 millimeters in length and 6 millimeters in diameter, resembling the larva of the palm weevil, except that the hinder end of the body is evenly rounded. The head is shiny and but slightly darker than the rest of the body, the region around the mouth and the mouth parts appearing dark-brown. A very thin, brown median line runs from the upper lip halfway to the back of the head. The spiracles are very small and almost biack. The surface of the body is smooth and very much folded. A few bristle-like hairs are seen on the head. (See Pl. X, fig. 1, illustrating a full-grown larva.)

Pupa.-The pupa is $\mathbf{1 3 . 5}$ millimeters long and 6 millimeters in diameter and of the same color as the larva. The surface is smooth and shiny. On the head, thorax, and dorsum of the abdomen there may be seen a series of stout, brown bristles arising from brownish tubercles. The tip of the abdomen has a small, white tubercle on each side, from the point of which arises a small, black bristle. There is little difference between the size of the tubercle and that of the bristle. 
The pupa rests in a cavity in the live wood, there being no attempt at forming a cocoon. (See Pl. X, fig. 3, giving a lateral view of the pupa.)

Adult (PI. X, fig. 4.).-The adult both in form and in size appears very much like the willow weevil, Cryptorrhynchus lapathi Linn., of the United States, except that there are no tubercles on the thorax and wing covers. It measures 11 millimeters in length (exclusive of the bill, which normally is doubled under the body) and is 5.5 millimeters in its greatest breadth. (Pl. X, fig. 4.) It is of a dark-reddish brown, somewhat mottled with gray on the forward part of the thorax, which is very closely punctured. The head is globular and fits almost entirely into a cavity in the front of the thorax, so that, when seen from above, it has the form of a thin crescent. The eyes are black and somewhat oval, nearly meeting on the front of the head, the space between being one-fourth the width of the rostrum or bill. When the insect is at rest the antennæ, which are inserted on each side of the rostrum near its base, are completely hidden, being drawn within the cavity in which the head fits. They are geniculate, the apical part or flagellum being somewhat more than half the length of the bill, very slender at its base, and increasing in size toward the club-shaped apex, which has three segments very closely united. There are 12 segments in the antennx, of which 11 are in the flagellum. (Pl. X, fig. 2.) The surface of the 3 apical ones is very pilose and of a sensitive nature. The rostrum is smooth, closely punctured, and slightly broadened at its apex. The mandibles are plainly visible, slightly darker than the rostrum, and uniting at their apices to form a triangle. The part immediately above the mandibles is covered with strong, light-brown bristles, pointing toward the tip of the rostrum. The thorax bears a longitudinal depression which is light-gray in color, owing to the scales on the surface, and which extends nearly to the posterior margin, where the depression becomes a ridge or carina one-sixth the length of the thorax.

The elytra reach nearly to the tip of the abdomen, are very rough, and are traversed longitudinally by nine rows of punctures forming very deep grooves; six of these extend to the apex of the wing covers, the others being interrupted or running into each other. The external (ninth) row terminates before the middle of the elytron. The posterior portion of the propluræ shows a decided depression, into which the front legs fit on each side.

The legs are moderately long and very stout, the fore pair being nearly a half longer than the other two. The rostrum reaches beyond the insertion of the first pair; there is a transverse carina of the mesosternum against which it rests. Two spines are situated on the under sides of the femora near their apices, the smaller nearer the apex, and those on the forelegs larger than those of the middle and hind pairs. The tibiæ are all of the same shape, each bearing a curved spine or tooth and 3 bristles at its apex; the latter are external.

The tarsi are 4-jointed, the fourth being very small and hidden between the pulvilli or pads. The tarsal claw is bifurcated, very long and slender. The tarsi are covered with long, blunt, silvery-white scale-like hairs.

The exposed part of the pygidium, or hinder segment of the abdomen, is bluntly, almost emarginately rounded; the apical half is covered with golden-brown bristles lying flat. The last ventral abdominal segment is hairy apically. The beetles appear to present no external sexual characters.

Remedies and preventives.-Doubtless these insects would be susceptible to the same general treatment as that given to the Asiatic palm weevil, although too little is known of their habits to be certain. 'They have been found in all stages, generally in diseased trees or in those debilitated by the attacks of other insects, and hence should not form a serious menace. 
TIIE BOT̃A WEEVIL.

Cyrtotrachelus sp.

This weevil lives principally in the trunks of the betel palm, Areca catechu Linn., where it does great damage, but inasmuch as it has also been found in considerable numbers in coconut trees, it is here described as far as its habits and appearance are concerned. In addition to the larvæ of the rhinoceros beetle and the cocoons and grubs of the palm weevil, one frequently encounters in decaying betel palms or coconut trees of 6 to 8 years, other smaller cocoons not more than 35 millimeters long and 15 millimeters in diameter. (Pl. VII, fig. 4.) These are composed of a more finely comminuted fiber than those of the palm weevil, and upon opening appear to contain a dwarfed example of the pupa of the Asiatic weevil. However, this pupa differs in many respects from that of Rhynchophorus ferrugineus Fabr., and the frequent finding of beautifully marked weevils of very small size convinces one that these cocoons and pupæ belong to the former.

$E g g .-$ No eggs have been encountered, and attempts at confining the adults for the deposition of eggs under conditions as nearly natural as possible have failed.

Larva.-The full-grown larva is nearly of the same size as the preceding one. However, in form it is more like that of the Asiatic palm weevil and is probably somewhat closely related to it. The color is a light-ocher yellow. The head is very much darker, and the mouth parts are dark-brown.

The length is 20 millimeters and the diameter 6 millimeters near the rear third of the body, the form being strikingly like that of the Asiatic weevil in this particular. The head projects forward and is smooth and shiny, with but few hairs scattered over it.

The spiracles on the first thoracic segment are larger than any of those on the other ones of the body, with the exception of the last abdominal segment, on which they are well developed and placed on the posterior aspect. The apex of the last segment is somewhat flattened and its hinder margin is prolonged into 4 rather obscure tubercles, from each of which arise 2 bristle-like hairs pointing posteriorly and slightly downward. Certain areas on the skin of the entire body, except the head, are rough or very minutely shagreened, single isolated hairs arising from some of them.

The mouth is minute, but the upper and lower lips and the mandibles are well developed, the latter being black. The palpi are prominent. As with the larvæ of Rhynchophorus ferrugineus Fabr., there are no evidences of external eyes or ocelli. (Pl. XI, fig. 1.)

Pupa.-The pupa is illustrated by fig. 3 on Plate XI. Its length is 13 millimeters and its greatest diameter 6 millimeters. It is of a whitish-ocher color, certain of the tubercles being a darker ocherous. On the front of the head, just above the point where the eyes would appear in the adult, there are 2 prominent corrugated tubercles, each with a single bristle; anterior to these are 2 smaller ones with bristles; and on the snout or rostrum above the antennæ are 2 more. On the front margin of the thorax are 2 other tubercles, smaller than the largest on the head; on the anterior half of the thorax toward the sides is another pair; and near the posterior margin are 2 others slightly larger; all of these are provided 
with a single bristle. The first 6 dorsal abdominal segments are very sharply defined and bear on each side of the middle a transverse group of 3 tubercles with bristles; outside of these is a single one.

The spiracles are plainly seen at the latero-dorsal angle of each segment. The pygidial segment is curved downward and at its middle there is a transverse line of 8 tubercles with bristles, slightly separated on the median line. The apical part of this segment has a median, transversely corrugated carina. On the extremity of the last ventral segment, on each side, there is a concentrically corrugated tubercle, from which 2 yellowish ochraceous bristles arise.

Each femur, on the outer part of its apex, lias a single tuberculated spine, darker than the surface. The pupæ are very active when taken from their cocoons, wriggling continuously if held in the hand.

A peculiar large, button-shaped spiracle may be seen on eaclı side just behind and a little below the prothoracic shield.

Adult.-This insect is graceful in form and very delicate in color. A black and white drawing, such as fig. 4 on Plate XI, of course can not show its coloring, which is one of its most striking features. The profile is shown by Plate XI, fig. 6 .

The length is 17 millimeters from the tip of the snout to the tip of the abdomen, and the diameter about 4.75 millimeters. Seen from above, it measures 13 millimeters in length. Its color is a combination of ocher, reddish-ocher, and darkbrown, or black, in which reddish-ocher predominates.

The head, exclusive of the snout, it globular, and smooth above and below, with a few scattering shallow punctures at the side. Its color is reddish-ocher. The eyes are jet-black and broadly crescent-shaped, nearly uniting at the upper and under sides of the head; their exterior outline, when viewed directly from in front, is almost a perfect circle. The rostrum is cylindrical and strongly curved downward, the basal third being twice the diameter of the rest and covered with circular light-gray spots, from each of which arises a tiny, darkbrown tubercular spine. The apical two-thirds is smooth and finely punctured longitudinally. The tip is slightly swollen laterally and of a darker color, as are the mouth parts. The mandibles are black and glossy, and tridentate, the teeth of one fitting into the interstices of the other. The narrow transverse labrum, with the anterior margin rounded, is scarcely visible. The antennæ, apparently composed of 8 joints, of which the first is equal in length to the others combined, are placed in short deep furrows on each side of the snout a little less than one-fourth the distance from its base. The upper edge of this furrow projects at its middle and somewhat overlaps the articulation of the first antennal joint. The last joint is greatly swollen, being twice the diameter of the preceding one, and is securiform. The length of the antennæ is equal to that of the rostrum. The third joint is chalice-shaped and one-half longer than the second one, which is inserted on the inner apical portion of the first. The prothorax is subconical, three-fourths as wide as the elytra, perfectly truncate at its anterior margin, and slightly rounded posteriorly. A narrow collar extends around its entire anterior margin, the sides of which are subparallel. The sides of the thorax are rounded, and their surface is smooth, dull, and sparsely punctured, the punctures toward the sides bearing small tubercles or spines. These punctures are also found on the underpart of the pro- and mesothorax, the metathorax, the ventral surface of the abdomen, the pygidium, the femora and the tibiæ of all the legs.

Thoracic markings occur as follows: A lancet-shaped, light-ochraceous or buff median mark, extending from a point behind the anterior margin one-fifth the length of the thorax, to the posterior margin; on each side of this a wide black line, broadening perceptibly at the posterior margin, meeting in front of the 
median mark and extending to the anterior margin; outside the black lines, on each side, a reddish-ochraceous one, twice as broad as the median and not extending to the anterior margin; external to these on each side, a broad pale-ochraceous or buff line, mixed with reddish-ocher at its outer edge and running imperceptibly into the extreme outer longitudinal black lines on the sides of the thorax. The black lines are irrorated with buff and have buff punctures anteriorly.

The scutellum, between the bases of the elytra, is lancet-shaped, black, and shiny.

The elytra have a ground color of reddish-ocher, with the following longitudinal markings: A series of 9 punctured lines, 5 of which reach the apex; a buff line on the sutural margin and a similar, although redder, space between the fourth and fifth and the sixth and seventh punctured lines. Each space is interrupted several times before it finally meets with the others near the apex of the elytron. The elytra bear near the base $\mid r$ and $\gamma \mid$ and on the apical half $\gamma \gamma$ on the left and right, respectively. There is also a broad, black, uninterrupted band on the external margin, confluent with a similar one on the prothorax. In the male these black characters are more or less confluent. The apices of the wing covers are emarginate at the suture; the pygidium is truncate, with the sides gradually converging; the median portion of the ventral surface of the thorax and abdomen is black and glossy, with numerous spine-bearing punctures; the posterior margins of the meso- and metathorax are deeply notched; the fore coxæ or first leg joints are almost contiguous, the interspace having a transverse suture. An elytron of the female is shown by Plate XI, fig. 5 .

The legs are stout and moderately long; the femora are slightly swollen at their apices; the tibiæ of the middle legs are somewhat shorter than those of the fore and hind ones, and all are longitudinally ribbed with spine-bearing tubercles of minute size. The apices of all tibiæ bear a large tooth and two stout bristles. The 4-jointed tarsi are covered sparsely above and densely beneath with golden-brown hairs. The bidentate claws are long and graceful. These beetles have no constant external evidences of sex differentiation. (See Pl. XI, fig. 2, showing hind legs.)

Remedies and preventives.-The same preventive measures and remedies apply in combating this insect as are recommended in the case of the Asiatic palm weevil. The damage done by them is not by any means so extensive as that due to the other insect, but, nevertheless, it should, if possible, be prevented or stopped, as the tree is finally killed by the summation of the attacks of the various insects which it harbors.

\section{THE FOUR-SPOTTED COCONUT WEEVIL.}

The length of this beetle, exclusive of the snout, is 5 millimeters, and the width is 1.5 millimeters. It was found in the dead or decayed heart or the undeveloped leaves of a small 3-year-old coconut tree during a search for the rhinoceros beetle. It attacks only dead trees of a very small size and is met with only in coconuts. In addition to the adult beetles, the larvæ and the pupæ were secured in numbers. Plate IX, figs. 1 and 2 , shows the exit holes of the adults and the work of the larvæ in the interior of the tree.

Egg.-The egg of this species is not known. It is probable that the beetles deposit their eggs on the sticky sides of their galleries in the trees, though close search failed to reveal them; but, as these places are also occupied by many 
other refuse-destroying insects and mites, it is probable that few of the shells would remain after the young grubs had emerged. Doubtless some of the mites feed upon the eggs themselves and in this way serve to limit the number of the beetles.

Larva.-The larva is of about the same general shape as that of the shot-hole coconut weevil, but it is more slender in proportion to its length. The length is 7 millimeters and the width 2.5 millimeters. The color is a pale-cream, the head being somewhat darker and the mouth parts dark-brown. The head bears numerous scattered, golden-brown bristles. The posterior margin of the labium is rounded, with a sharp angle at the median line. The ventral surface of the first thoracic segment is microscopically and densely spinose-tuberculated, as are also certain transverse areas on the ventrum and dorsum of the middle abdominal segments. The spiracles are extremely minute, somewhat slender, and pyriform, with lines radiating from the central slit to the margin. The upper, posterior surface of the last abdominal segment is slightly excavated, with 10 rather fine bristles; it is of a golden-brown on its margin. The larvæ feed in well-defined burrows or galleries slightly isolated from each other.

Pupa.-The length of the pupa is 5.5 millimeters and the width at the middle 1.75 millimeters. It is cream-colored and in general shape like the pupa of the shot-hole coconut weevil. Golden-brown spinose hairs are arranged as follows: Two pairs, very small, on the rostrum above the antennæ, 2 larger ones in front of the eyes, 2 still larger ones on the top of the head back of the eyes, 8 pairs symmetrically on the prothorax, 7 on the meso- and metathorax, respectively, 2 on each abdominal segment from the first to the sixth, 1 on the seventh, 1 at the base of the pygydium, and 1 on the ventral apical margin of the last abdominal segment, pointing downward. Each femur is provided at the outer apical angle with a single erect spinose hair. The spiracles are hardly visible.

Adult.-The general color of the beetle is dark-brown, with rufous patches. The head is globular and strongly punctured. The eyes are black and broadly crescent-shaped, contiguous beneath the head, but separated above by a narrow shallow sulcus at the base of the rostrum. The rostrum is slender, subcylindrical, slightly swollen laterally at the base above the insertion of the antennæ, and coarsely punctured, each of these punctures as well as all others upon the surface of the body containing a single club-shaped hair or bristle. ( $\mathrm{Pl}$. X, fig. 6.) The mouth parts are extremely minute. The mandibles are tridentate, and when closed are almost completely hidden within the mouth cavity. A narrow longitudinal sulcus is situated on each side of the mentum, into which fit the maxillary palpi. The antennæ are 8-jointed and of the same length as the rostrum; the first joint is slightly shorter than the other seven, the last is double the diameter of the preceding one and its distal half is silvery-pubescent with sensitive hairs.

The thorax is truncately conical and its anterior and posterior margins straight and parallel, the former having a narrow, smooth collar, back of which are numerous setigerous pits or punctures. It is coarsely and deeply punctured, with an indistinct rufous spot on each side. The scutellum is subtriangular and excavated at its middle. Each elytron is marked by 2 reddish-subquadrate spots, one at the base and the other beyond the middle, and is traversed longitudinally by 5 very finely punctured ridges or carinæ. Between every 2 carinæ there is a double row of very regular, coarse, deep punctures. The apex of each elytron is rather sharply rounded, the pygidium is subtriangular, and its sides and median line are carinated and rather densely setose, the setæ springing from fine punctures. It is very easily depressed; in some specimens it forms an angle of nearly $90^{\circ}$ with the remainder of the abdomen. The legs are stout, moderately long, and the pairs about equidistant from each other and from the 2 extremities of the body, roughly dividing the latter into 4 subequal sections, if the rostrum 
is excluded. The femora are swollen toward the apical angle and each has a slight constriction beneath and just in front of the apex. Each tibia has an apical claw and a tuft of pre-apical hairs beneath. The tarsi are all of equal length. The legs are conspicuously shiny in comparison with the rest of the body. Plate $X$, fig. 8, shows profile and fig. 7 an antenna of this beetle.

As is the case with nearly all weevils, these also feign death when annoyed. They conceal themselves under any available object and unless disturbed remain in one spot in their burrows for a long period. ${ }^{*}$

Remedies and preventives.-These beetles are found only in locations where others have preceded them and killed the trees; hence, they are not in any sense a menace to the healthy tree. Their description has here been given merely to call attention to all forms which may be encountered.

${ }^{4}$ It was hoped that the identifications of the shot-hole coconut weevil, the bonga weevil, and four-spotted coconut weevil would be received from Washington in time for insertion in this article, but as they have been delayed, it was thought best to publish the paper and give the identifications later.

$36540-9$ 


\section{ILLUSTRATIONS.}

\section{Plate I.}

Fia. 1. Jigg of Oryctes rhinoceros 1.; magnified portion shown at $1 b$.

2. Head of larva. (X 5.)

3. Antenna. (X 12.)

4. Left mandible of female:

(a) Profile and inner surface, showing condyle. (X 7.)

(b) Interior view. (X 7.)

5. Profile of head and thorax of female. (X $1 \frac{1}{2}$.)

6. Profile of head and thorax of male. ( $X l_{\frac{1}{2}}^{\frac{1}{2}}$.)

\section{Plate II.}

Coconut showing results of attacks of Oryctes rhinoceros L. and Rhynchophorus ferrugineus Fabr.

\section{Plate III.}

Fia. 1. Crown of coconut, showing inverted cone, in longitudinal section, eaten out by larvæ of $O$. rhinoceros and $R$. ferrugineus Fabr. (About oneseventh natural size.)

2. Adult of 0 . rhinoceros L. boring into petiole of coconut leaf. (Onehalf natural size.)

\section{Plate IV.}

Fig. $\quad$ 1. Larva of Oryctes rhinoceros L.

2. Pupa of Oryctes rhinoceros L.

3. Adults, male and female, of 0 . rhinoceros L. (All about natural size.)

\section{Plate V.}

Figs. 1-4. Heart of coconut showing burrow made by adult of 0 . rhinoceros, and a female beetle working at center of tree. (One-half natural size.)

\section{Plate VI.}

Fig. 1. Egg of Rhynchophorus ferrugineus Fabr.; magnified portion shown at $1 b$.

2. Head of larva of same. (X 21 2 .)

3. Labium of larva of same. (X 3.)

4. Diagram of work of larvæ in base of coconut trunk, showing points of entrance, as at $\mathbf{A}$.

\section{Plate VII.}

Fig. 1. Larva of Rhynchophorus ferrugineus Fabr.

2. Pupa of Rhynchophorus ferrugineus Fabr.

3. Cocoon of $R$. ferrugineus Fabr., showing beetle partly emerged.

4. Cocoon of Cyrtotrachelus sp. (All about natural size.).

\section{Plate VIII.}

Fia. 1. Adults of $R$. ferrugineus Fabr. (About natural size.)

2. Adults of Rhynchophorus sp. (About natural size.) .

3. Work of larvæ of $R$. ferrugineus Fabr. in wood of coconut near roots. The larvæ entered from the lower left. 


\section{Plate IX.}

Figs. 1, 2. Work of shot-hole coconut weevil in trunk of coconut, with exit holes of adults.

Prate X. (1)rawn by W. Schultze.)

Fici. 1. Larva of shot-hole coconut weevil. (X 3.)

2. Antenna of shot-hole coconut weevil. (X 3.)

3. Pupa of shot-hole coconut weevil.

4. Adult of shot-hole coconut weevil. (X 4.)

5. Adult of shot-hole coconut weevil, profile. (X 4.)

6. Adult of four-spotted coconut weevil. (X 8.)

7. Antenna of same. (X 36.)

8. Profile of same. (X 8.)

Plate XI. (Drawn by IV. Schultze.)

Fig. 1. Larva of Cyrtotrachelus sp. (X 5.)

2. Hind legs of adult Cyrtotrachelus sp), (X 10.)

3. Pupa of Cyrtotrachelus sp. (X 5.)

4. Adult male of Cyrtotrachelus sp. (X 5.)

5. Elytron of female of Cyrtotrachelus sp. (X 5.)

6. Profile of male of Cyrtotrachelus sp. (X 5.) 


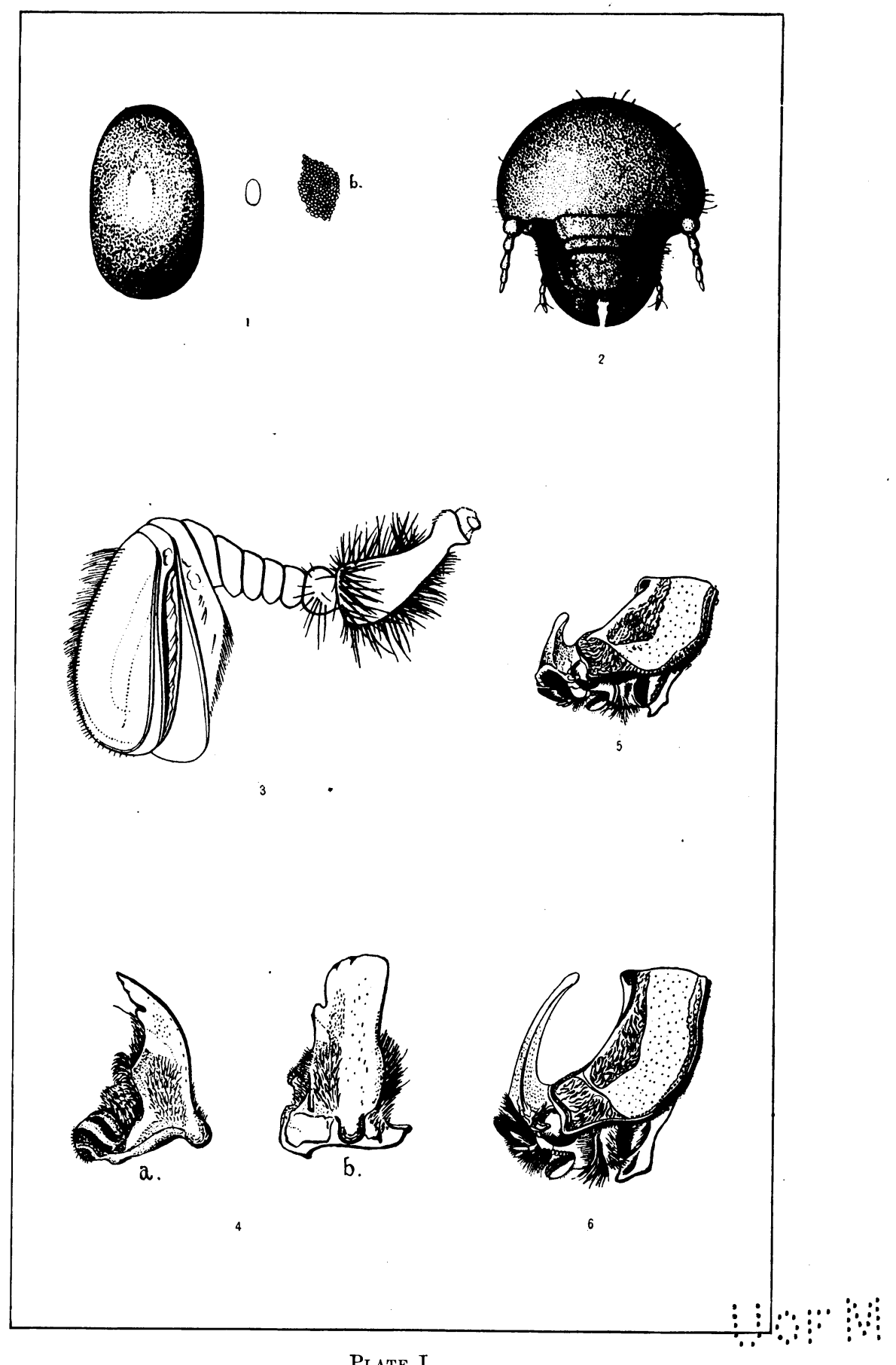

Plate I. 


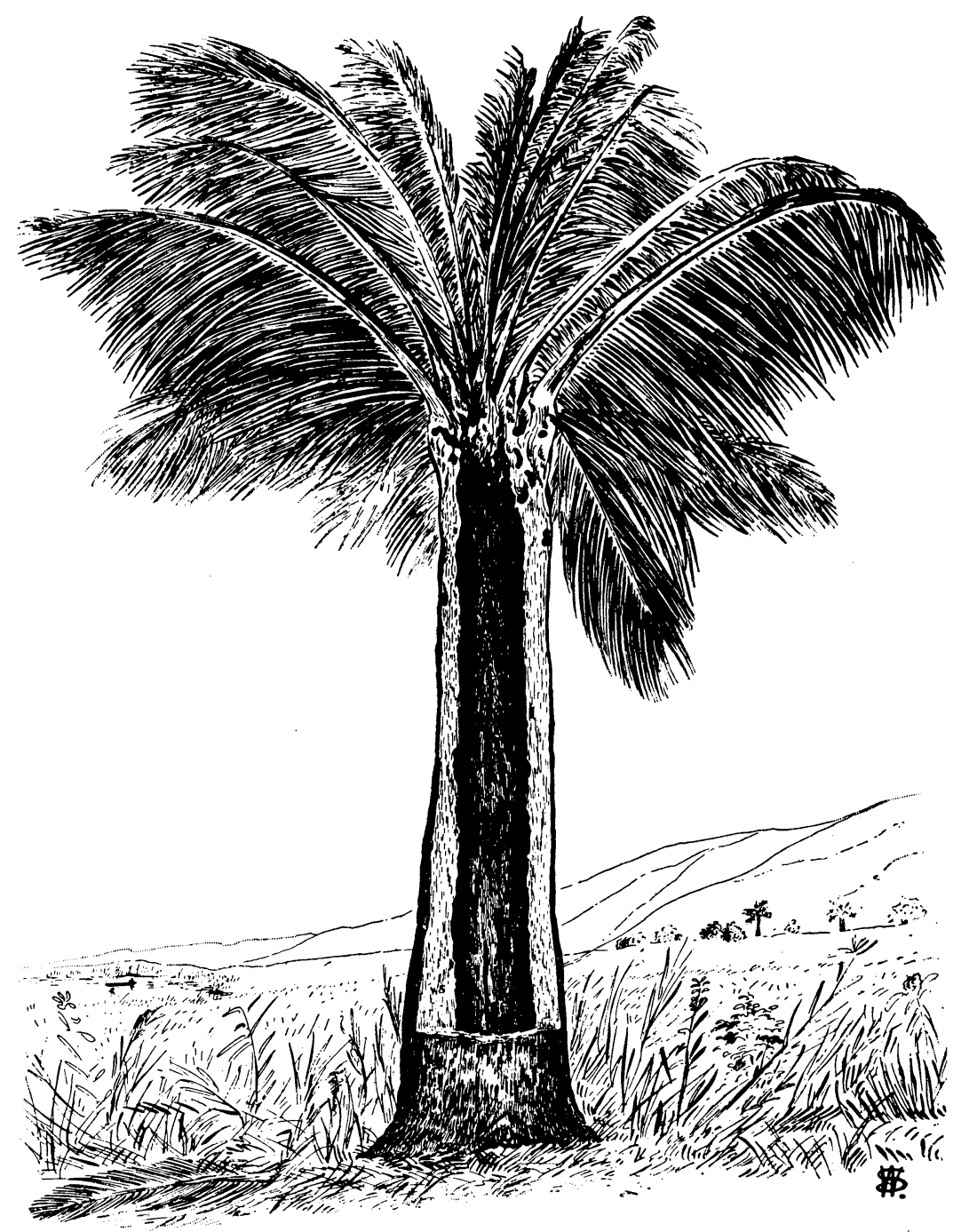

Plate II.

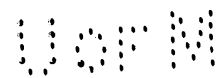




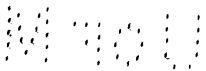




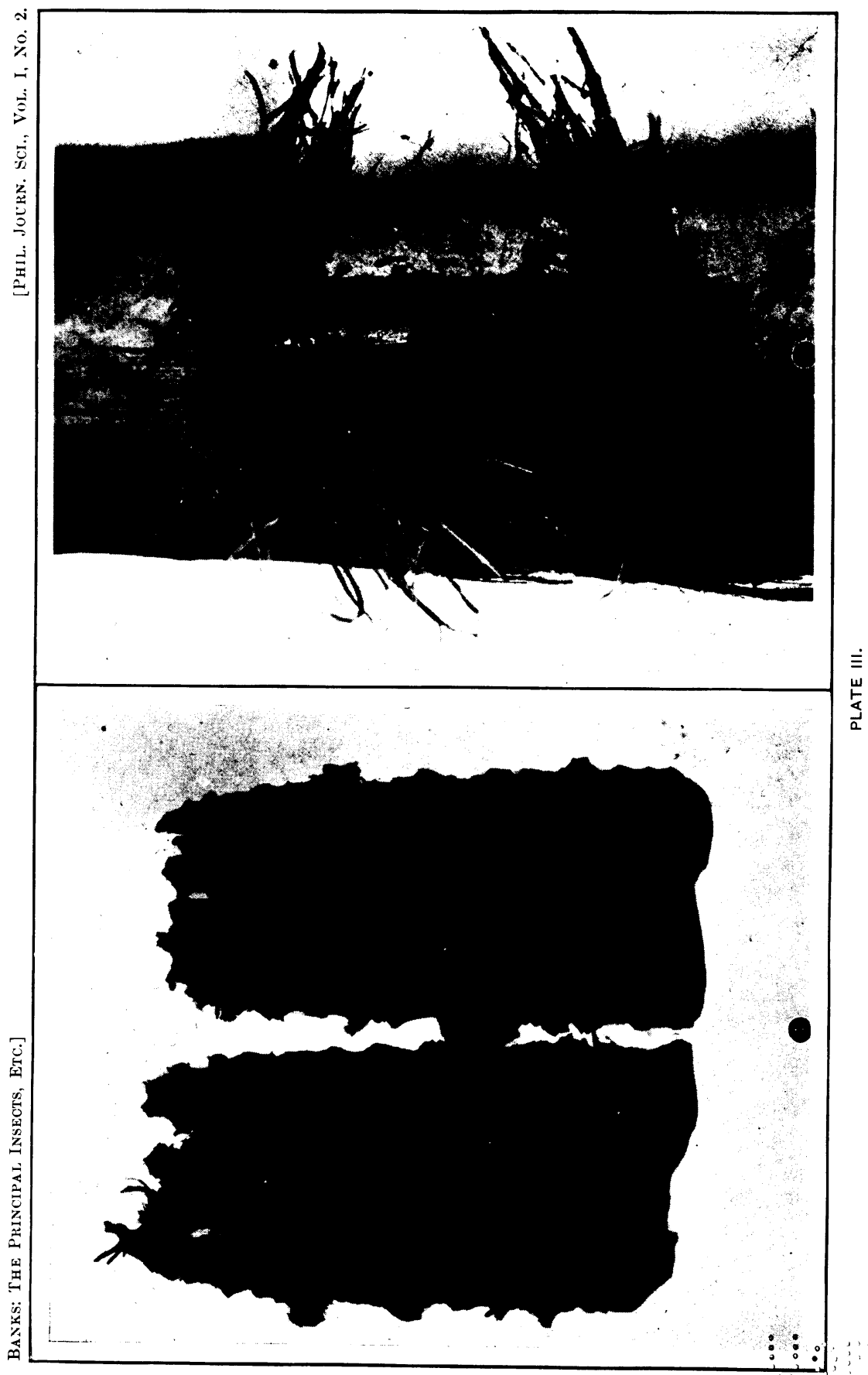




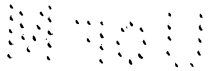




$$
\overbrace{40}^{8}
$$


: 


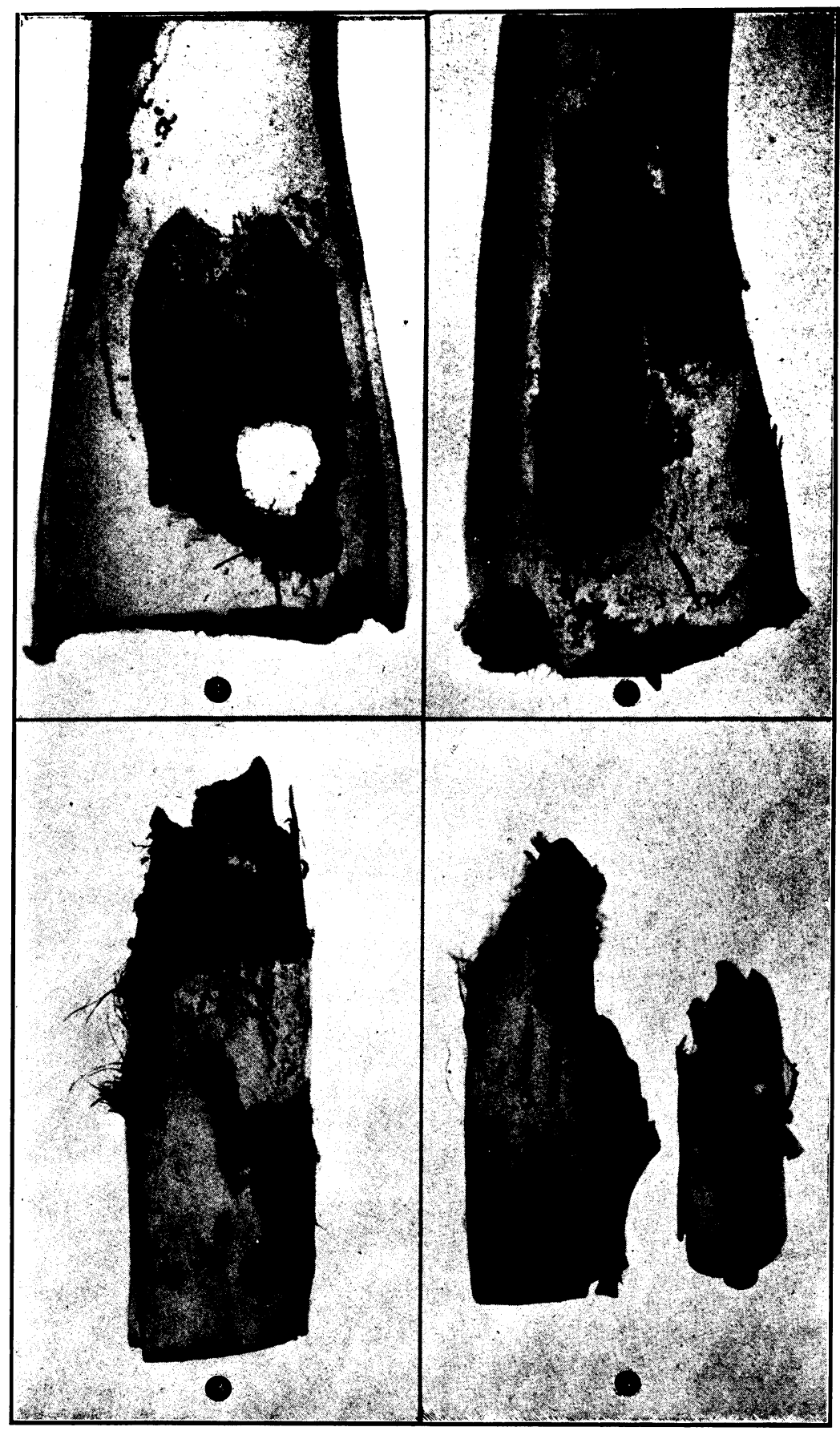

PLATE V.

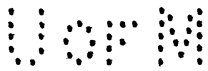





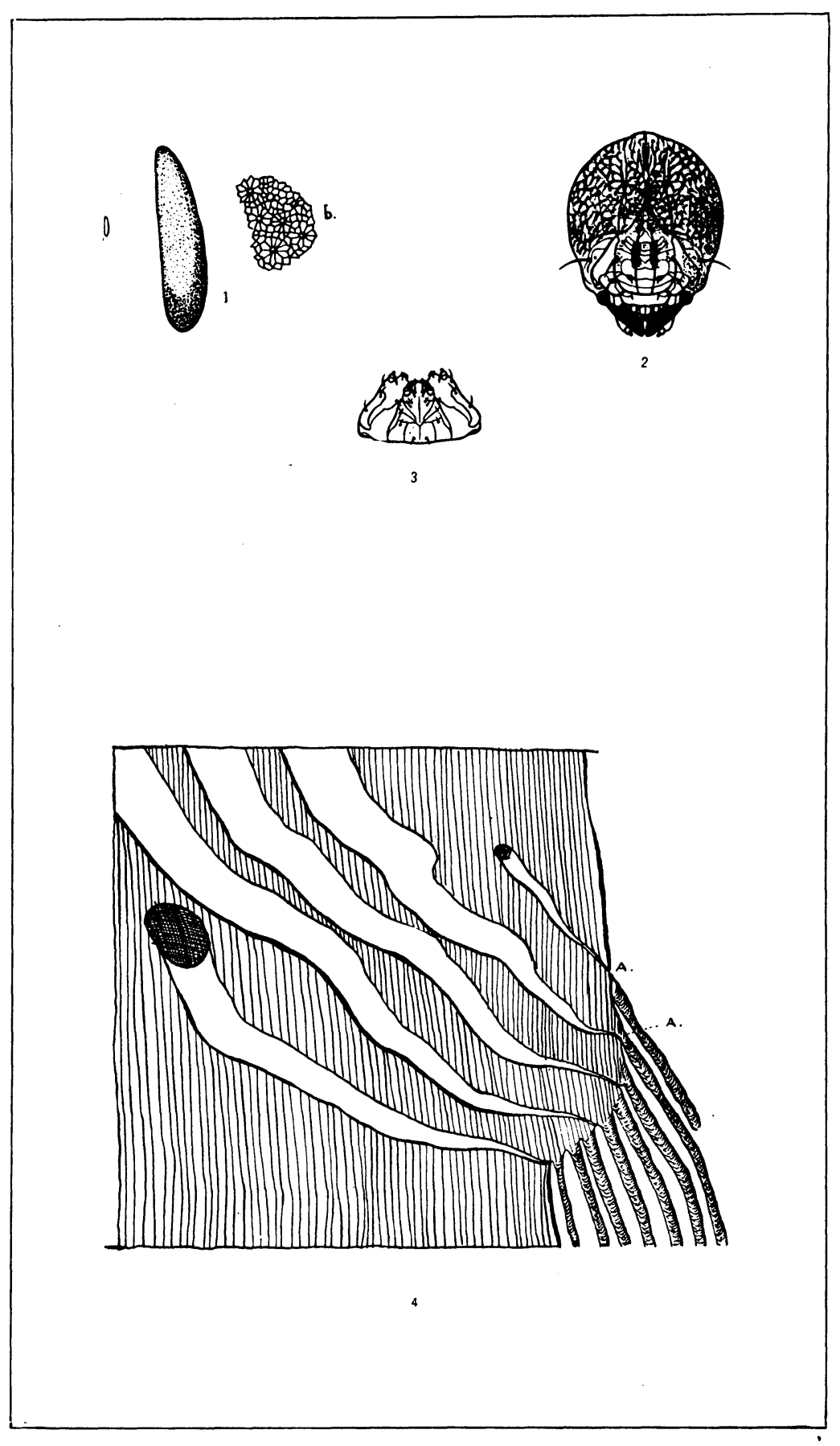

Piate VI. 
.

a $\cdots \vdots$ 


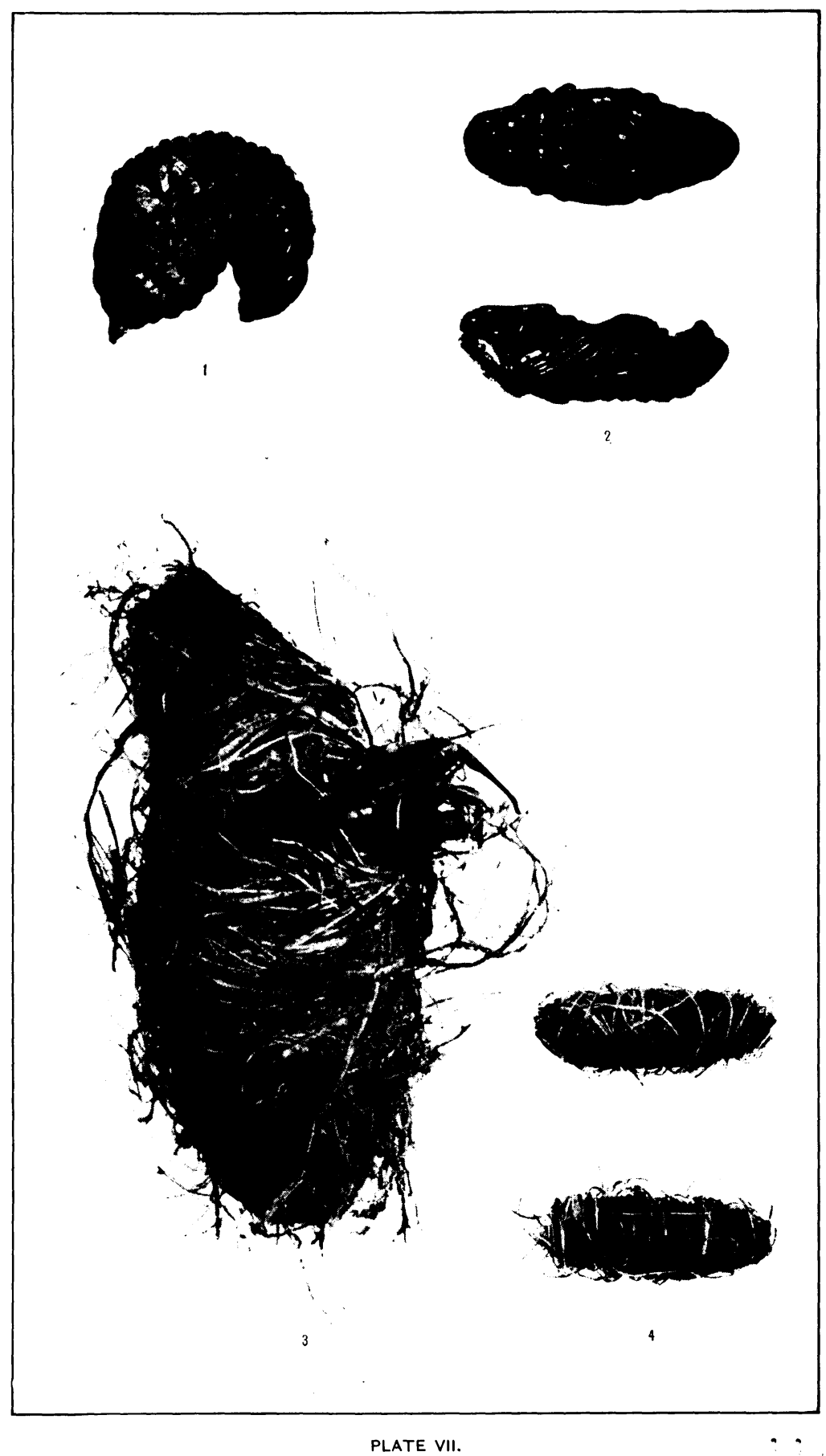


(1) 


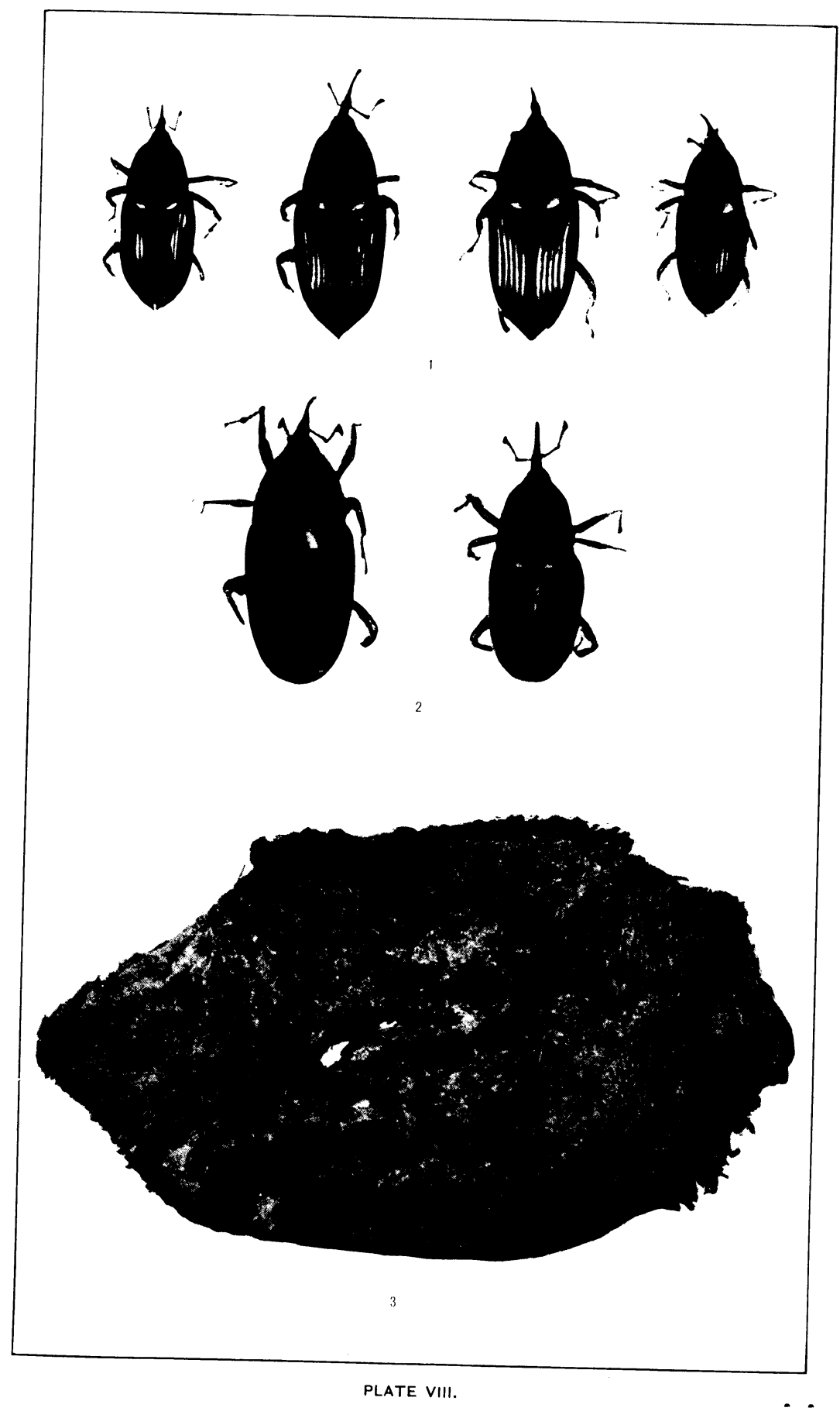




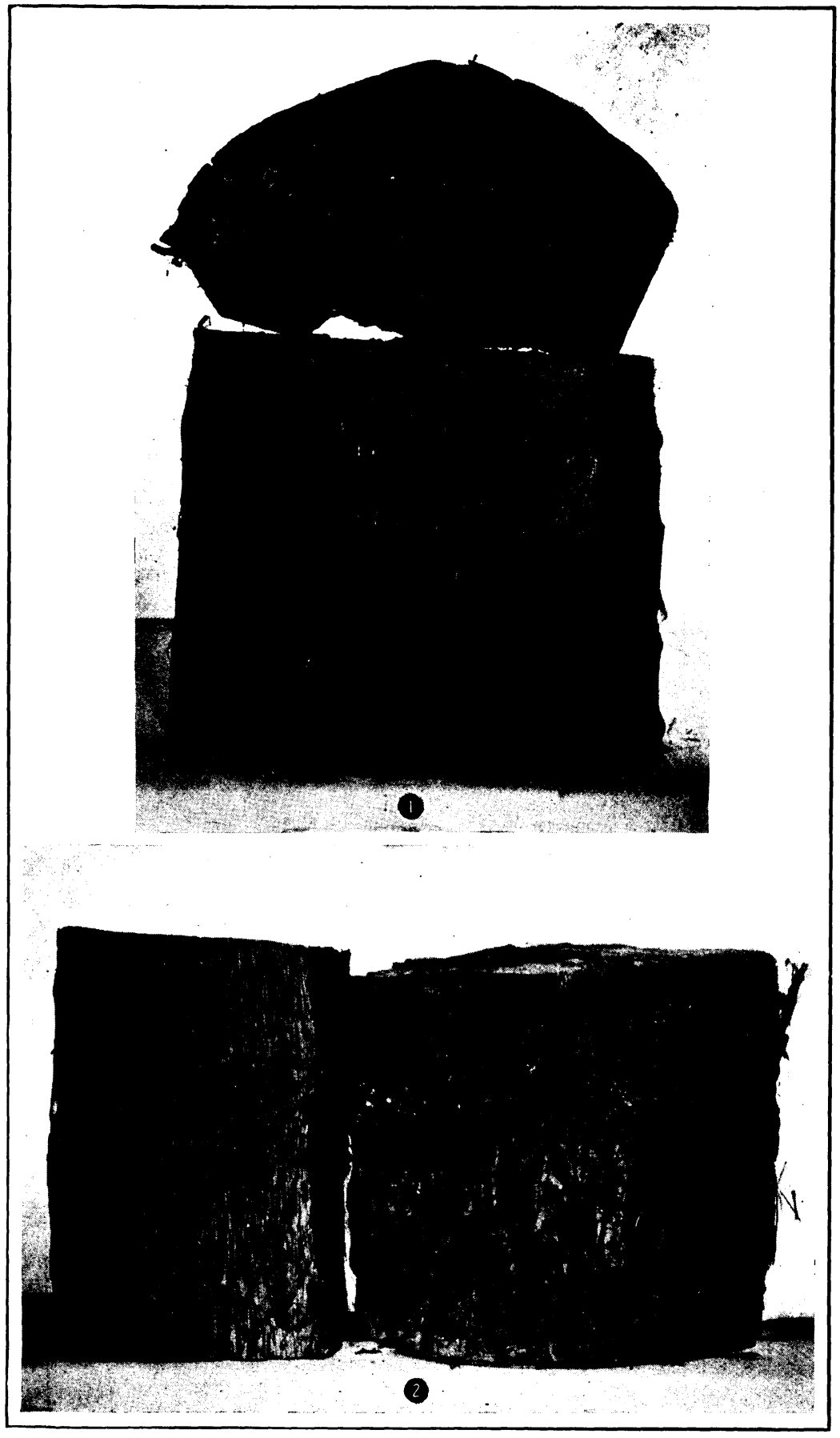

PLATE IX. 
: : . . 


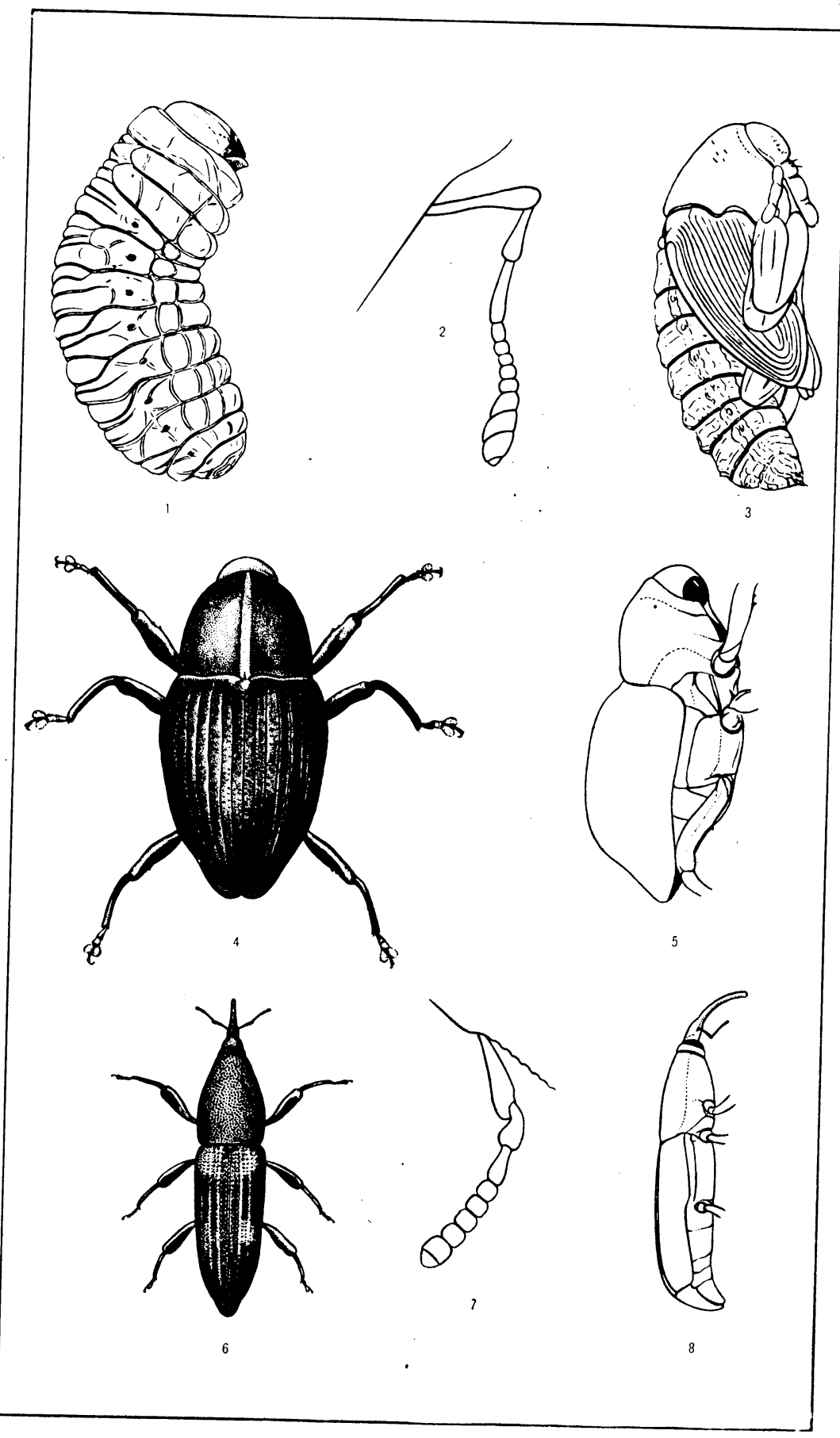

Plate $\mathrm{X}$. 


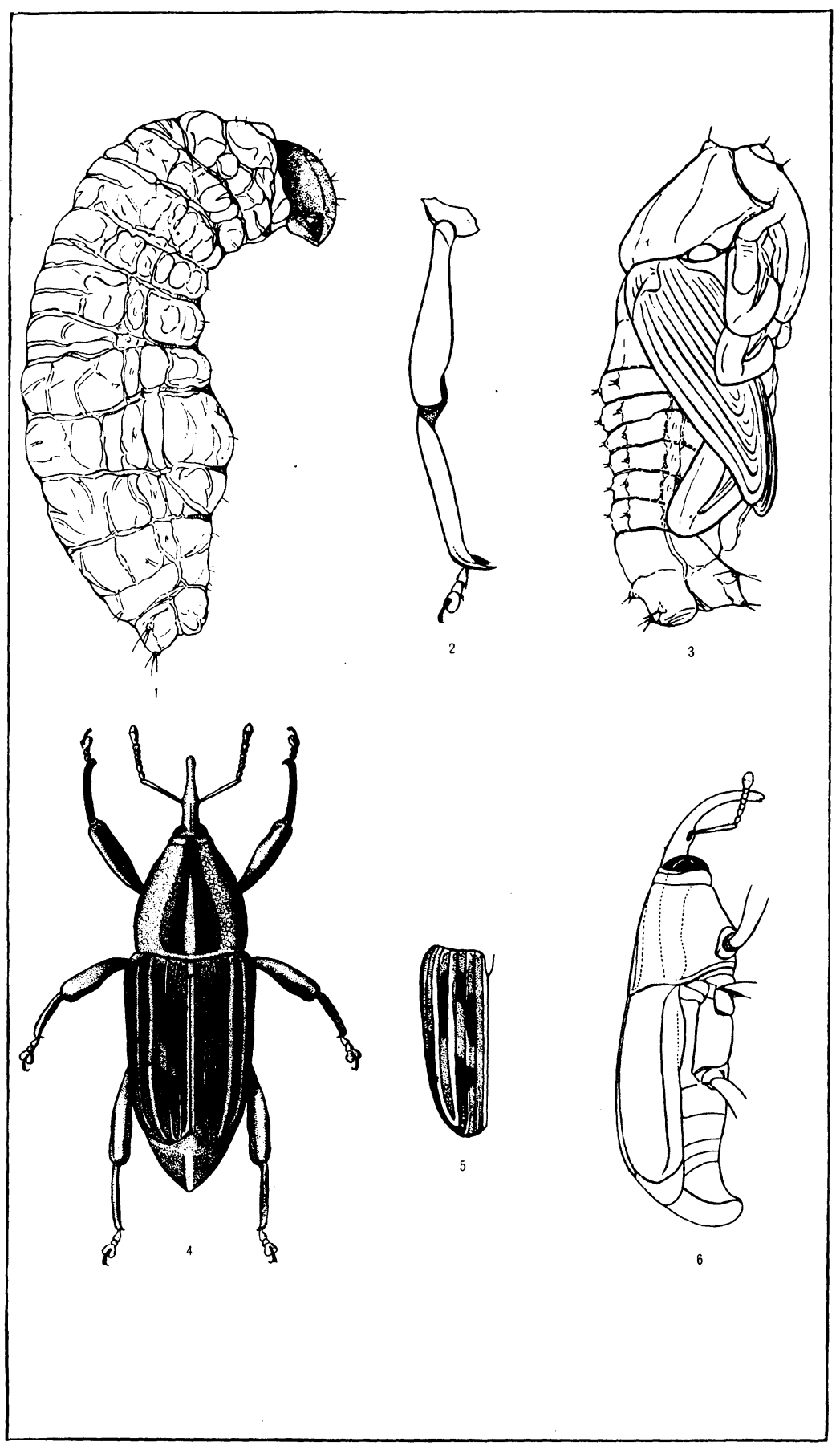

Plate XI. 
( 


\title{
THE PRINCIPAL INSECTS ATTACKING THE COCONUT PALM (PART II).
}

\author{
By Citarles S. Banks.
}

(From the Entomological Section of the Biological Laboratory, Bureau of Science.)

In Part I of this paper, insects which attack the trunk and the undeveloped leaves and flower clusters of the coconut were discussed. All the forms which have been described belong to the Coleoptera, but there are also certain species of Lepidoptera and Coccidæ which attack the coconut to a sufficient extent to warrant their being designated as injurious. $^{1}$

Two forms of Lepidoptera are found upon the leaves of the coconut, one belonging to the Rhopalocera and the other to the Heterocera; the first is the coconut skipper, Padraona chrysozona Plötz, of the family Hesperiidæ, and the second, Thosea cinereamarginata Banks, of the Limacodidæ. While the nature of the damage done by the caterpillars of these two forms is very similar, the insects differ entirely from each other both in the larval and adult stage. Neither is likely to prove a very serious menace to the life of the tree. Each attacks the leaflets after they are practically full grown. A single caterpillar confines itself to a single leaflet until, with the exception of the midrib, it has entirely devoured it, whereupon it proceeds to another, and so on until the caterpillar has attained full growth. In the case of the coconut skipper, the caterpillar not infrequently eats a space from the blade of the leaflet at a point near its attachment to the main petiole, leaving the distal part untouched. (See Pl. I.)

${ }^{1}$ The bibliography of coconut insects, appended, includes all forms known to attack the tree, either here or in other countries, and is intended to be of further aid to those interested in the subject from an economic standpoint. 


\section{THE COCONUT SKIPI'ER.}

JAPIDOPTERA.

IIESPERIID $/$.

Padraona chrysozona Plötz.

During the months of September and October many of the leaflets of small coconut trees of from 6 to 15 feet in height are partially destroyed. Certain of these leaflets have their outer edges sewn together by means of a pure-white silk which is decidedly elastic, so that the leaf may be pulled slightly apart without tearing the fastening. Inside these folds the light, yellowish-green caterpillar, having a chitinous head, somewhat darker than the body and boldly marked with a very regular pattern, is encountered.

Toward the latter part of October the semiactive pupæ are found in these "cradles," partially covered and surrounded by a snow-white flocculent substance, which has a wax-like feel. This substance has very much the appearance of the wax secreted by certain species of Coccidæ and is exuded from the skin pores of the caterpillar toward the end of its larval stage. It serves as a protection for the pupa.

The coconut skipper, like nearly all Hesperiidx, flies during the very early morning or the late afternoon and early evening hours, and hence it is very difficult to observe its egg-laying habits.

The eggs are found upon the under side of the leaflets of the coconut and but rarely more than one occurs upon a single leaflet. They hatch. in from seven to eight days and the young caterpillar, after devouring all of the eggshell except that portion in contact with the leaf surface, at once proceeds to the edge of the leaf and begins to feed. This process consists in cutting out an oblique swath extending toward the midrib, of about the width of the insect's head. Frequently the caterpillar abandons a portion of the leaf, after having fed upon it for a short time, the result being that leaflets are encountered the margins of which are deeply notched, as shown by Plate II, fig. 1. Under normal conditions, and after the caterpillar has cut the leaflet to the midrib, it sews the margins together to form its nest, feeding upon the cut edge, either toward the apex or the base of the leaflet.

The neck of this caterpillar is much constricted, and therefore the head has considerable freedom of motion, but in a state of repose the normal dorso-ventral axis of the head is so inclined that it lies nearly in a plane with the longitudinal axis of the body, thus causing the mouth to be elevated and projected forward to form the extreme anterior point of the insect (Pl. II, fig. $3 \mathrm{~A}$ ), which, in such larvæ as those of Attacus atlas Linn., and Thosea cinereamarginata Banks, is formed by the front of the face or the occiput. The caterpillar of the latter has the head deflected beneath the body. 


\section{Description and Life History.}

Egg (Pl. 1I, fig. 2 and 2 A.) -Diameter 1.65 millimeters, height 0.85 millimeter ; of a rather flat, oblate-spheroidal shape, yellow-glabrous when first laid, with crimson, subcentral ring and central spot covering the micropyle, and developing after two days. The under surface nearly flat, glabrous; upper surface minutely punctured. It adheres strongly to the leaf surface.

The eggs of Padraona chrysozona Plötz are always laid singly upon the leaf, thus differing from those of Erionota thrax Fabr., which may be found in groups of from 8 , to 15 .

The period of incubation is from seven to eight days.

Larva (PI. II, fig. 3).-Length 3.5 millimeters, width of head 1.2 millimeters; upon emerging from the egg. At this stage the larva is of a pale, greenish-yellow, with a black head, the size of which appears somewhat disproportionate to that of the body. A very fine, light-grey, sparse pile covers the body, especially the posterior segment.

The full-grown larva measures 45 millimeters in length and 4.5 millimeters in width, the head being 3.75 millimeters in dianeter. It is of a pale, ocher-green, semi-transparent, permitting the viscera, especially the heart and the malpighian and urinary glands, to be seen readily through the skin. The head, which is about one-tenth the length of the entire body, is biscuit shaped or of a very flat, oblatespheroidal. The surface is strongly and coarsely punctured. The ecdysical sutures are strongly marked by narrow sulci. It is of a glabrous, tawny, fleshcolor. A dark-brown line extends from the base of each mandible around the side of the head to the occiput, where it is deflected forward, following the ecdysical suture and being again deflected toward the side of the head, ending in a sharp point, the lines of each side thus forming a $Y$ on the median, dorsal aspect of the head. The ocelli, which lie in the beginning of the dark line posterior to the mandibles, are 6 in number on each side and of a dark-brown. The mouth parts are dark-brown and glabrous and are surrounded by a rather coarse, porrect pile. The anal segment is glabrous and its posterior margin is strongly rounded, with numerous, white, curved setæ projecting from it posteriorly. The legs are light yellowish-buff with many white setæ on their lower surfaces. The abdominal feet, of which there are 10, are strongly pubescent. The spiracles, which are functional on the 1st and 4th to the last body segments, are of a light-yellow.

Pupa (Pl. II, fig. 4 and $4 \mathrm{~A}$ ).--Length 25 millimeters, width 4.5 millimeters. The pupa is of a glabrous, dark-brown, but is frequently so covered with a white, flocculent substance that its true color is not apparent. It is strongly seto-pilose, especially on the anterior dorsal part of the head and thorax and on the abdominal segments. The setæ upon the thorax project anteriorily, those upon the abdomen posteriorly. Very dark-brown rings extend around the apical margins of the 4 th, 5th, and 6 th segments. The proboscis extends to the apex of the 6th, its apical fourth being force free and traversely rugose.

A very remarkable feature of this pupa is the form assumed by the prothoracic spiracles. They are completely protected by a reniform patch of dense setæ and are located one on each side of the posterior dorsal margin of the pronotum. See Pl. II fig. 4 B.

Imago, male (Pl. III, fig. 1).--Length of body 15-16 millimeters, length of fore-wing, 15.5-17.5 millimeters. Ground color, bright, yellow-ocher, with the following dark-brown markings or suffusions: The veins and a more or less obliterated longitudinal patch along the posterior part of vein $\mathrm{VII}_{1}$, and $\mathrm{VII}_{2}$, from the base for one-half its length, in some specimens suffused with yellow-ocher; a similar, somewhat wider patch from the end of the cell to a point its own length from the outer margin. In some specimens this patch is confluent with the basal 
one, forming an irregular, oblique band across the dise of the wing from the base to near the apex. Exterior to this wide patch, a yellow-ocher, irregularly rhomboidal spot, divided by the dark-brown vein $\mathrm{V}_{2}$. A broad, marginal, dark-brown band from the costa to the posterior angle of wing, its inner margin scalloped and its area crossed by ends of veins which show yellow-ocher; a dark-brown suffusion at the base of and posterior to vein IX. Cilia, black, suffused with yellow-ocher toward the apex and yellow-ocher toward the posterior angle. The inner margin clothed with long yellow-ochraceous hairs. The hind wings with ground color dark violet-brown at the base, with iridescent, subcostal scales. Margin, darkbrown; cilia, yellow-ocher. A longitudinal, yellow-ochraceous patch in the cell, from which spring long hairs of the same color. A broad, yellow-ochraceous, irregular patch extends across the wing from the outer third of vein III to the middle of vein IX, which it follows to the base and margin of the wing respectively. Posterior to the cell patch is another one of long, yellow-ochraceous hairs extending to the oblique band. A similar patch extends along the inner submarginal area its own width from the inner margin of the wing.

The under surfaces of wings yellow-ocher; veins, marginal hair line, base and inner margin to vein IX and submarginal series of suffused spots on fore wing, dark-brown. On the hind wing the superior, oblique, yellow-ochraceous patch has its margin indicated below by series of faint brown spots or irrorations. Antennæ brown, with dark yellow-ochraceous tips and lighter scales beneath. Palpi, sternum, venter, legs and apical margins of abdominal segments, yellow-ocher. Apex of abdomen dark violet-brown with yellow-ocher irrorations. Removal of the head in both sexes reveals a patch of very broad, pearl-colored scales dorsally at the neck. Under normal conditions these scales are completely hidden by the hairs upon the head and thorax.

Female (Pl. III, fig. 2).-Length of body 17.5-20 millimeters, length of wing 18.5-21 millimeters. Ground color, dark violet-brown, especially on the veins of the wings; iridescent in certain lights.

The bases of the fore and hind wings, the head, thorax, the abdomen dorsally and the entire lower surface suffused with greenish-buff. Fore-wings above, with an irregularly, sub-rhomboidal spot in the end of the cell, 2 oblique, parallel lines between this and the costa, 3 parallel patches beyond them, near the costa an oblique, interrupted band, from the outer third vein of $V_{3}$ to the middle of inner margin, buff, irrorated with brownish scales. Beyond the oblique band an irregular patch between veins $I_{1} I_{6}$ and $V_{2}$ interrupted by vein $V_{1}$. Inner margin with greenish-buff hairs for two-thirds its length. Cilia brown and greenish-buff.

Hind-wings as described above, with an ill-defined, interrupted, oblique, buff band from the middle of vein V to the middle of vein IX along which it extends as in the male. Cilia, buff. Inner submarginal area with long, greenish-buff hairs.

Beneath; wings brown, entirely suffused with greenish buff; the markings, which are buff above, are very pale-buff below and both wings have distinct, darkbrown veins and marginal lines.

Antennæ dark-brown above, and buff beneath to the base of club; bases of antennæ, black. Palpi, greenish-buff; apical segment, dark-ocher. Thorax and legs covered with dark, greenish-buff scales and hairs; tarsi, with reddish-brown spines beneath. Abdomen with 5 distinct, transverse, black lines and 3 indistinct apical orange ones dorsally. Anal region with orange scales.

Semper ${ }^{2}$ says of this species: "This beautiful species is very easily recognized by the 3 yellow rings of the abdomen. It belongs next to augiades Felder and palmarum Moore, in which the males also lack the sexual sign [discal patch], upon the forewings, which appears in the female of augias L., and bambusa Moore.

${ }^{2}$ Semper: Reisen auf den Philippinen (1892), 2 No. 5, 301 et seq. 
Upon this ground, I believe, Staudinger's opinion that bambusa is a variety of augiadcs can never be right according to my way of thinking."

"The female of chrysozona varies greatly in the width of the dark markings; 1 have some which are as dark upon the upper side of the forewings as Moore's palmarum, others again have, with exception of the border, only fine, black stripes along the veins and a faint, dark shadow on the end of the cell. The ground color is darker than in palmarum, and the same as in augiades. The female on the upper side is exactly the same as the drawing of palmarum, but differs on the underside in that the light bands are nearly as clearly marked as they are above. The ground color is greenish-gray-brown."

This species is also found upon the betel palm (Areca catech $u$ L.) ; in fact, Semper indicates in his note concerning this insect that the larva is only encountered upon that tree. My observations disprove this statement; indeed, it rarely is seen on any palm other than the coconut.

Preventives and remedies.-This insect is never found in sufficient numbers to justify the fear that it may become a serious menace to coconut culture, but as its feeding upon the leaves of small trees may have a tendency to debilitate them, its larvæ should be destroyed whenever they are encountered.

Parasites.-This insect is probably, to a great extent, held in check by two small Hymenopterous parasites, Chalcis obscurata Walk., and an unidentified Braconid, both of which attack the larva, laying their eggs within its body, their young feeding upon its fats and body fluids. The larvæ of the former parasite, of which there may be as many as 10, pupate within the pupa of the coconut skipper, which they kill, emerging therefrom in from five to six days thereafter (Pl. IV, fig. 1); those of the Braconid leave their host when they are full grown and, like all true Braconidæ, they spin pure-white cocoons in the vicinity of the now dead and shriveled caterpillar. After spinning their cocoons the insects emerge in about 4 days. (Pl. IV, fig. 2.)

DESCRIPTION OF PARASITE.

Walker's description of the Chalcid is as follows:

Chalcis obscurata Walk., Proc. Ent. Soc. Lond. (1874) 399.

Male.-Body, antennæ and legs black, with the usual structure. Body convex. Head and thorax scabrous, dull. Antennæ stout, nearly filiform. Prothorax about four times as broad as long. Sutures of the parapsides, distinct. Abdomen smooth, shining, subsessile, with cinereous tomentum toward the tip. Femora yellow at the tips, hind femora minutely denticulated beneath. Tibiæ yellow, striped beneath with black; hind tibiæ black at the base. Tarsi yellow, tips black. Wings cinereous; squamulæ yellow; veins black; ulna about half as long as the humerus.

Hab.: Hiogo (George Lewis), Philippines (Banks).

This is the first record of this species of the Chalcididæ as from the Philippines. 


\section{THE COCONUT SLUG-CATERPILLAR.}

\section{LEPIDOPTERA.}

LIMACODII) A.

T'hosea cinereamarginata Banks.

Thosea cinereamarginata Banks, Phil. Jou'n. Sici. (1906), 1, No. 3, p. 229.

The slug-caterpillar is easily distinguished from other Lepidopterous larvæ by the form of its body and its mode of locomotion, which is more like that of slugs or snails than of insect larva. Several species are known in the Philippines, many of them feeding upon palms. In Manila, this caterpillar is quite common and is usually found feeding either upon the upper or the lower surface of the leaves of the coconut. It presents a rather forbidding aspect, due to its being well armed with a double series of spinous tubercles placed upon either side, but, as a matter of fact, unlike most Limacodidæe, it possesses no poisonous properties. I have handled the larvæ freely without experiencing any discomfort.

The damage which this insect does to the coconut leaves is about equal to that of Padraona chrysozona Plötz.

Egg.-Diameter 1.5 millimeters, height 0.95 millimeters; of a flat, oblatespheroidal shape and with minute reticulations upon the surface, pale-ochraceous. The larva escapes through a slit which divides the shell across its face, and the latter is not eaten as in the case of Padraona chysozona Plötz. The period of incubation is from 5-7 days.

Larva (Pl. V, fig. 1).-When full grown, length, 23.75 millimeters, width, 14.25 millimeters including the tubercles. It is pale-green above and pale greenish-yellow at the sides, being almost pure-white beneath, and with the following markings: a median, light-purple or heliotrope band with symmetrical scalloped margin, the scallops expanding upon the respective segments. The margin of this band is darker purple and shades into the green of the dorsum. The band is developed into more or less of a patch upon the fourth and seventh segments, where the colors are darker. External to this band, on either side, is a series of 9 horizontally-projecting, spiniferous tubercles, those upon the second, fourth, sixth, eighth, and eleventh being twice or slightly more than twice as long as the remaining ones. Below these, ventrally on either side, is a series of 8 light-purple spots, one on each segment from the third posteriorly, and below these another series of 8 spots beginning upon the 4th segment. At the latero-ventral angle, a series of 11 horizontally projecting spiniferous tubercles, one of which projects anteriorly and another posteriorly on either side. The spines of these tubercles interlace and are yellowish-green at their bases and purple or black at their tips. Many of the spines have a white hair at their tips, and the shorter ones at the bases of all tubercles have somewhat inflated tips. The head is yellow-green and when the caterpillar is not feeding, is retracted within the lst thoracic segment. The length of the larval stage varies from 21-25 days.

Pupa (Pl. V., figs. 2 and 2 A.) - Length 8.5 millimeters, width 5.5 millimeters. The color is a light yellow, with ochraceous bands on the posterior margins of the dorsal, abdominal segments; the wing pads are livid flesh-color and the eyes are dark-gray. 
Cocoon.-Length, 8.75 millimeters; dark-brown, cocciform or oblate ovoid, composed of finely comminuted leaf fiber held together with silk. The interior is white and silk lined. The pupal stage lasts about 22 days. Time of flight, the month of January.

Adult.-A description of the adult male and female of this species occurs in the Phil. Journ. Sci. (1906) 1, No. 3 p. 229 . It is quite closely related to Thosea minima Semper, from which, according to Semper's description, it differs chiefly in having the prominent antemedial oblique sinuate band extending from near the cell spot to the middle of the inner margin.

Preventives and remedies. - The same methods of treatment apply to this species as to the Coconut skipper, though, as in the case of the other, there is no possibility of its ever becoming a serious pest.

\section{SCALE INSECTS.}

With exception of a few species from which useful or commercial products are obtained, such as the cochineal insect, Llaveia cacti Linn., and the lac insect, Tachardia lacca Kerr, practically all known species of scale insects are detrimental to man's agricultural interests. In some parts of the world they do more damage to crops and trees than is due to the effects of all the other insects of the region. While, in the Philippines, this is not so strikingly true in the case of the coconut palm, still the damage done to this tree by species of the family Coccidæ is very considerable. It is rare to find a coconut which does not, by its yellow or brown leaves, indicate the ravages of these pests. Scale insects differ so greatly from ordinary insects that they may easily escape detection. As a rule, the commoner forms appear merely as rusty-brown or yellowish patches upon the surfaces of the leaves, or on the bark of the stems or trunk of the plant.

Characters.-In all species the body of the adult female is either covered with a scale formed of a waxy secretion in which the exuviæ of the earlier stages are compacted or else the body of the insect itself assumes a form which suggests a scale or tubercle upon the host plant. The males of all species are winged, but on account of their very minute size and pale colors escape notice unless they are bred upon the food plant under glass, in which case they may be captured upon emerging as adults. The newly hatched young of both sexes are, of course, much smaller than the adults of either sex and it is almost impossible to see them with the naked eye.

Upon hatching, the young, coming from beneath the parent scale, scatter upon the leaf surface in quest of a favorable place to settle. Shortly after their "first meal, which is obtained by inserting their probosces into the succulent part of the leaf or twig, the insects shed their skins, but during the period of feeding there will have exuded from certain body pores a pale, wax-like secretion which, adhering to the first exuviæ, after a brief period assumes the form of a scale-like covering. 
In the first molt the females usually shed their legs and sometimes their antennæ, so that a female nymph or adult appears as a mere sack, attached to the plant by the proboscis. The female, in those species having a distinct scale, remains under this covering throughout life, while the male, after a succession of molts, comes forth with legs and wings well developed.

In view of the great difference in appearance between the male and the female, it is necessary to consider their respective characteristics separately for purposes of classification, it being impossible to identify two given specimens of different sex as belonging to the same species unless they are found in close association or are bred from a given lot. In view of the relative scarcity of males in most genera of Coccidæ, the characters found in the female form the chief basis upon which their determination is made.

In the Philippines, so far as is known, seven species of Coccidæ are found upon the coconut. Of these, Aspidiotus destructor Sign. is by far the most abundant and destructive; next in abundance is Chrysomphalus propsimus Banks, a species which has usually been encountered in great numbers on all trees examined both in Manila and in the provinces. 'The order of abundance of the remaining species is that of the following notes :

\section{THE TRANSPARENT SCALE.}

Aspidiotus destructor Sign.

This extremely prolific scale is found on the coconut palm in all localities in the Archipelago where investigations have been conducted. It is extremely injurious to the trees, causing their leaves to assume a characteristic yellow color, which is easily noted from a distance. Where it is encountered, the under surfaces of the leaflets are covered with thousands of small, rough, circular patches, which are almost transparent and so thin that the insect and her eggs can be seen beneath. When the leaflet is pulled longitudinally or when it wilts, the scales become striated owing to the tension on the edges which are attached to the leaflet. Plate VI, fig. 1, shows adults and young scales upon a leaflet. It will be noted that the latter have fixed themselves to the longitudinal veins and therefore are arranged in very regular rows. Fig. $1 \mathrm{~A}$ shows young insects which have emerged from beneath the scale of the parent; female scales from which the occupants have been eaten by a tiny predaceous beetle of the family Coccinellidæ, are also present. Fig. 2 shows a coconut leaflet attacked by a form of disease which causes spots, very similar to those resulting from the attacks of Aspidiotus destructor Sign., to appear on the upper surface. In case of doubt as to the origin of the spots, certainty is reached by examining the underside of the leaflet, where, if it is attacked by scale insects, the latter will be found just at the point of discoloration of the leaflet. 
DESCRIPTION.

Egg (Pl. VII, fig. 4).- - Length 0.2 millimeter, width 0.1 millimeter, regularly, bluntly oval, one side more convex, very pale lemon yellow, smooth, laid in two or three more or less regular concentric rows around the parent within the scale. This regular distribution necessitates a nearly complete rotation of the female around the point of insertion of the proboscis. This is accomplished by an undulating motion of the body and may be observed by placing the live insect upon a piece of glass, under the microscope.

Larva (PI. VII, fig. 8).- Inmediately after hatching the length is 0.25 millimeter, the width 0.15 millimeter. Pale yellow, slightly lighter in color than egg. Eyes dark red; antennæ 5 jointed, slightly setose, last joint 3 times as long as the first 4 ; transversely, microscopically striate, biapical as shown by Plate VII, fig. 5, with a single seta from each apex; mouth two-fifths of distance from frontal to anal margin. Anal margin dentate, giving indication in both sexes of existence of pygidial lobes. These disappear in the male upon the second molt. Legs moderately long, femora somewhat stout. Tarsi single jointed with 2 knobbed spines on the dorsal margin. Proboscis about as long as the body. Four minute hairs project from the frontal and 2 from the anal margin of body, the anal being 4 times the length of the longest frontal.

Male puparium.-Oblong-oval, pale, translucent, larval exuviæ at center slightly darker, yellowish. Plate VII, fig. 3, shows the male puparium.

Female puparium (PI. VII, fig. 2).-Differs from male in being more nearly circular. Color as in male. Larval exuviæ at or slightly removed from the center. Darker than the scale itself, yellowish.

Signoret's description ${ }^{3}$ is very meager. It is as follows:

"The scale is round, of a transparent white, with the exuviæ at the center, and of a yellowish transparent white.

The female is yellow, round; the extremity with six lobes, of which the two median are shortest; the pygidium with four groups of wax glands of eight to ten orifices in each [group] agglomeration."

He says further concerning the insect: "This species appears to cause great damage to coconut groves in the Island of Reunion, where they are menaced with complete destruction. The scale is also found on palms and dates. We have found it likewise on Goyavius psidium [Psidium guayava] which we received in the same package."

It will be seen that Signoret's description is not sufficiently detailed to differentiate this scale from other very similar ones, as he makes no mention of the squames, their number, and arrangement, which, for example, is a very important point in distinguishing Aspidiotus destructor Sign., from A. latania Sign. The following description has been prepared from fresh material:

Adult female (PI. VII, fig. 2) .- - Length 0.80-0.90 millimeter, width 0.65-0.75 millimeter, bright pale-yellow, broadly oval, nearly circular, narrowed posteriorly, with slight emargination at the base of the pygidium, which is only slightly paler; posterior margin, whitish creamy, due to waxy secretions; two submedian white spots on each side of the genital aperture show the position of the circumgenital glands, the posterior of which have 4 to 5 apertures, the anterior 7 to 9 . Anterior margin of the body regularly rounded, abdominal segmentation laterally distinct. Antennæ, small, oval knobs with inwardly curving bristles at the apex, situated one-third of the distance from the frontal margin to the rostrum. Between the

${ }^{3}$ Ann. Soc. Ent. de France (1869), (4) 9, 120; Plate XII, figs. 8 and 8a. Translation. 
antennæ on the ventral surface are from 5 to 10 minute spinous tubercles. Parastigmatic glands not present. Stigmata subcylindrical. Pygidium (Pl. VII, fig. 6) with 6 lobes, median pair shorter than the next, weakly tricuspid, lightbrown, next pair bicuspid, slightly paler, exterior pair of the same color, bicuspid, of the same length as the median. Chitinous portions of all lobes run anteriorly for some distance into the pygidial area, the surface of which is closely, longitudinally striate. Squames as follows for each side: 2, apically fimbriated, between the median lobes, similar ones, but more slender, between lst and $2 d$ lobes, 3 stouter between $2 \mathrm{~d}$ and $3 \mathrm{~d}$ lobes, with fimbriation somewhat externally laterad of apex; a series of 9 broad, laterally fimbriated ones beyond the $3 \mathrm{~d}$ lobe and extending one-third the distance from the latter to the base of pygidial margin. These squames decrease in length and increase in width from the third lobe and the number and length of their fimbriations decrease so that the last one bears but 1 prominent spine, the remainder being reduced to sharp serrations of its latero-apical margin. Setæ placed as follows: One pair at the external base of each median lobe nearly twice the length of latter, 1 pair between the second lobe and its 1st external squame, 1 pair ventrad to external lobe, 1 very short, ventrad to the 4th of the exterior 9 squames, 1 ventrad to the last squame, a small setose tubercle near the ventral margin, two-thirds of the distance from the last squame to the base of the pygidium. Four groups of circumgenital glands, the posterior pair with 4 to 6 orifices, the anterior with 7 to 12 . Tubular spinnerets filiform; their heads chitinous; tube obconical, chitinous; their tongues one-third the diameter of their heads and equal to them in length. Numerous trumpetshaped or subcylindrical ducts toward the apex and having orifices on the margin, as do tubular spinnerets. Anal opening about halfway from genital orifice to posterior apex. For details of structure see Plate VII, fig. 6.

Adult male (Pl. VII, fig. 1).-Pale-yellow, with darker, pinkish-yellow, transverse apodema. Head about one-tenth the length of entire body, including genital sheath. Ocelli very dark red; upper pair slightly extra-marginal, lower pair posterior and contíguous on median line, their diameter one-fourth greater than upper ocelli. True eyes posterior to and their own diameter distant from the upper ocelli, submarginal. Antennæ composed of 10 joints, of which the first 2 are subglobular, 3 to 5 subequal in length and about 3 times the length of second, all segments sparsely setose. Tenth segment attenuated, terminating in a clubbed hair, surrounded by 3 other curved hairs of equal length. This is shown by Plate VII, fig. 7. Legs moderately long, posterior femora slightly stouter than the others. The single jointed tarsi, two-thirds the length of tibiæ, slender, subconical and moderately covered with spinous hairs. Tibiæ subequal to femora, with a very few hairs each. Claws or ungues one-fourth the length of tarsi, their digitules two-thirds their length. Tarsal digitule as long as ungues. Genital sheath long and tapering to a sharp point; two-sevenths of the length of the rest of the body. Wings iridescent, hyaline, obovate; veins of about equal length and subparallel to the respective margins. Haltere has the first joint swollen to about one-third its length, just before the apex. Second joint of length equal to the first; spinous and hooked at extremity. Length of wing 0.63 millimeter. This insect so nearly resembles Aspidiotus lataniae Sign. that it is very difficult to separate the two species. The chief points of difference in the female are the number of squames external to the outer lobe, the number of orifices in the circumgenital glands, and the relative length of the median and second lobes of the pygidium, the median in A. destructor Sign., being shorter than the $2 \mathrm{~d}$ pair, while in A. latania Sign., they are of equal length and more markedly tricuspid. 
Aspidiotus destructor Sign., is by far the most pernicious of the scales which attack the coconut in the Philippines. It most frequently occurs on young trees having from one to five years' growth, in many cases completely covering the under surfaces of all the leaves, giving them a characteristic yellow tinge. It is certain that it is attacked by a Hymenopterous parasite, as female puparia have been found showing the exit holes of the adult parasite, but as yet the latter itself has not been discovered. A small Coccinellid beetle, Scymnus sp., is a voracious feeder upon the transparent scale, the adults as well as the larvæ of this species frequently being encountered in considerable numbers upon coconit leaves which are covered with the scales. A description of this insect follows :

Scymnus sp.

Larva.-Length 1.75 millimeters, width 1.01 millimeters, exclusive of the purewhite waxen tufts which project from the front, sides, and posterior margins of the body as shown by Plate VIII, fig. 2. The larva of this beetle when once known can easily be distinguished from all others which might be found among scale insects in this region. It is extremely active, running from place to place and greedily gnawing open the delicate scale in order to obtain the insect which lies beneath. The body is pale yellow with a greyish tinge.

Pupa.-Length 1.25 millimeters, of a light ocher-yellow. This insect pupates within the larval skin, as do many species of the family. In this case the skin splits along the median dorsal line, exposing the pupa.

Adult (Pl. VIII, fig. 1).--Length 1.35 millimeters, width 1 millimeter, of a dark brown, almost black, with a light-brown discal spot on each elytron. In some specimens this spot is sharply, in others ill, defined. The entire body covered with a fine, white, pubescence. Palpi, apices of femora, tibix and tarsi, brownish ocher. (See Pl. VIII, figs. 3 and 4 for antenna and palpi.)

Habits. This beetle, the adult as well as the larva, feeds on many species of Coccidæ but has been found in greatest abundance in colonies of Aspidiotus destructor Sign.

Chrysomphalus propsimus Banks.

Chrysomphalus propsimus Banks, Phil. Journ. Sci. (1906), 1, No. 3, p. 230. (Pls. I and II.)

This scale bears a general resemblance to C. aonidum Linn., but its color and size, together with its apparent predilection for the coconut palm, upon which it is always found, make its identification as a distinct species a matter of some doubt. The scales crowd themselves upon both surfaces of the leaves of neglected or deformed trees and frequently as many as 4 or 5 are found overlapping each other. In Manila they breed with great rapidity and soon cover the leaflets and even the petioles. The same species has been encountered in great numbers upon the betel palm (Areca catechu L.) at San Miguel de Mayumo, Province of Bulacan. It may be distinguished from other scale insects which might be found upon the coconut by the decided, shining red-orange color of the pellicles. The male scales are infrequently met with in comparison with those of the female. (See Pl. X.) 
This delicate, though prolific, scale is frequently seen in Manila upon young, badly cared for coconut trees. It is found upon the upper surfaces of the leaves and because of its flat shape and gray color is difficult to detect. While not as serious a menace as either of the foregoing species, it merits attention because of the possibility of its great increase if it is left unchecked.

Chionaspis candida Banks.

Chionaspis candida Banks, Phil. Journ. Sci. (1906), 1, No. 3, p. 232. (Pl. IV, figs. 1 to 5.)

Frequently coconut trees are found the partially opened leaflets of which are covered with small, pure-white spots, due to the scales of another species of insect differing totally as to color and form from the foregoing. This scale multiplies rapidly upon either surface of the leaf, usually in the protected parts. As a rule, the female puparium occurs near to a group of male puparia or else with a group of the young scales in their first or second molt (Pl. IX). This insect is not as nomadic as Aspidiotus destructor Sign., therefore its opportunity for debilitating the tree is not as great and the danger from it is not to be feared in the same degree as from $A$. destructor Sign.

\section{Lepidosaphes megregori Banks.}

Lepidosaphes megregori Banks, Phil. Jour. Sci. (1906), 1, No. 3, p. 233. (I'ls. V and VI.)

This scale is comparatively rare. It occurs upon both sides of the leaves of the coconut, especially on old ones, but seems to prefer that part of the upper surface that is near to the midrib. It is always encountered singly and the puparia are seldom distorted as in the case with Chionaspis candida Banks. Although rare, it may at any time and under favorable conditions propagate to the extent of being injurious. The most noteworthy features which distinguish it are the pair of white, waxy, horn-like projections on the front of the first pellicle, the light color of the female puparium and the regularity of its transverse striæ.

\section{Lepidosaphes unicolor Banks.}

Lepidosaphes unicolor Banks, Phil. Journ. Sci. (1906), 1, No. 3, p. 234. (Pl. VII, figs. 1 to 7.)

This species is of nearly the same shape and size as the preceding, being only distinguished externally by the narrower, interior margin, the color of the puparium and the absence of the waxy horns in old specimens. It is less frequently met with than $L$. megregori Banks and therefore less likely to prove a menace. 
Paralecanium cocophylla Banks.

Paralecanium cocophyllae Banks, Phil. Journ. Sci. (1906), 1, No. 3, p. 235. (Pls. VIII to XI.)

This insect differs from any of the foregoing in that, if a female, it does not lie beneath a puparium but is itself its own scale. It has easily been found upon nearly every coconut examined in Manila, and is readily distinguished from other species by its unusual size (being 5 to 6 millimeters long and nearly as broad), and by the 2 small patches of orange-yellow on the posterior region. It always occurs upon the inferior surface of the leaf. The male puparia are much scarcer than those of the female.

A peculiar characteristic of the male insect is that it comes from beneath its scale to shed the pupal exuviæ, returning after it has completed its transformation. The length of time after the final molt and before it seeks the female, during which the adult male remains beneath the puparium is not known.

PREVEN'TIVES AND REMEDIES.

In all the work upon scale insects affecting the coconut, it has uniformly been observed that those trees which are ill cared for or which have become deformed by the attacks of beetles are the ones most infested by scales. The malformed or pathologically imbricated leaves, in their interstices, offer ideal places for the breeding of scale insects. This fact would point to the necessity of the removal and destruction of such portions at once.

Because scale insects can only migrate as wingless larvæ, it would seem that their arrest would not be difficult, and yet, when we consider that every wind blows these larvæ from leaf to leaf and from tree to tree, we can easily see that this fact, as well as the extreme fecundity of the insect, renders no tree entirely safe from their attacks. However, those trees which are the healthiest and best cared for are the ones which will best withstand these pests.

Spraying with lime-sulphur or kerosene emulsion washes might serve, if properly applied, for the preservation of young coconut trees, but these remedies would entirely be out of the question for full-grown ones. The necessity is apparent for clean, systematic and regular cultural methods for the protection of this valuable tree from scale, as well as from all other insect pests.

\section{INSECTS AFFEOTING COPRA.}

In connection with work which has been carried on in this Bureau on coconuts, copra, and coconut oil, it has been noted that certain lots of commercial copra, when received from the bodegas, were badly infested by larvæ, pupæ, and adults of Silvanus surinamensis Linn., and Necrobia rufipes De Geer. Both of these insects are cosmopolitan and as they 
feed upon stored products of a character similar to copra it is only necessary to mention them in this connection.

In the case of these, and of most other injurious insect forms, preventive measures are always most advisable and if care is taken in packing and shipping the copra, receptacles into which the insects can not find entrance being employed, their ravages will be prevented. It is difficult to exterminate them if once they secure a lodgment in a mass of copra. Carbon bisulphide, which might be used successfully against similar insects in grain, would probably prove detrimental to copra owing to its power of dissolving oil.

\section{BIBLIOGRAPHY.}

Blanford, W. F. H. Palm Weevil in British Honduras, Royal Gardens, Kew, $B$ Bul. of Useful Information (1893), LXXIV, $2 \tau$ et seq., plates 1 and 2. Reviewed by Riley \& Howard, Insect Life (1893), V, 357.

Bureau, E. Calandra palmarum, Ann. Soc. Ent. de France (1853) XXVII, 1.

Chittenden, F. H. The Principal Household Insects of the United States, $U$. S. Dept. Agr., Div. Ent. (1896), IV, n. s., 105 and 121.

Cockerell, T. D. A. Coccidæ or Scale Insects, Agric. Record (1892), Dec. Reviewed by Riley \& Howard, Insect Life (1893), V, 362.

List of Coccidæ observed in Jamaica, Insect Life (1892), IV, 333.

The West Indian Rufous Scale, Ibid. (1892), IV, 381.

The Food Plants of Scale Insects, Proc. U. S. N. Museum (1896), 19, 780 , Cocos nucifera L. Fifteen species of scale insects noted as occurring upon the coconut.

Some Coccidæ from the Philippine Islands, Proc. Davenport Acad. Sci. (1905), X, 133.

Coconut, Enemies of. Trop. Agr. (1889-90) IX (1890-1), X et vol. seq. Notes upon various injurious insects attacking coconut.

Coquerel, CH. Observations entomologiques sur divers Insects Recuilles á Madagascar. Sur les moeurs des Oryctès de Madagascar et sur deux espèces de Scolia qui vivent dépens des larves de ces Oryctès. Ann. Soc. Ent. de France (1855) III, 167.

Faune de Bourbon, île de la Réunion, Coléoptères, Ann. Soc. Ent. de France (1866) VI, 334.

CurTis, J. (Ruricola) Aleurodes cocois, Gardener's Chronicle (May 2, 1846).

De Mornay, C. F. Letter on Palm-beetles to Penang Gazette and Straits Chronicle (Sept. 24, 1889).

Fawcert, W. Report on the Cocoa-nut Disease at Montego Bay. Bul. Bot. Dept. Jamaica (Sept., 1891).

Correspondence on the Cocoa-nut disease. Ibid (May, 1892).

Ferguson, A. M. \& J. All about the Coconut Palm (1885).

Fernald, Mrs. M. E. A Catalogue of the Coccidae of the World (1903).

GreEn, E. Ernest. Coccida of Ceylon (1896), 95, 207, 218.

HickeY, JoHN B. Notes on the Palm Weevil (partly reproduced from Home and Farm, Louisville, Ky.) ; also note by editors Insect Life (1891), 4, 136.

HonN, G. H. Note on Larva of Rhynchophorus cruentatus. Trans. Am. Ent. Soc. (1878), VII, 39.

KonIG SBERGER, J. C. Ziekten van Rijst, Tabak, Thee en andere cultuur gewassen die door Insecten worden veroorzaakt. Meded. uit. s'Landsplanten. van Java (1903) , LXIV, 92 (1898), XXII, 31, 33, 39, 42. 
Phillits, J. H.; GabB, F. E., \& Bellamy, J. Report of Commissioners appointed by his Excellency the Governor to inquire into the destruction of the Cocoapalm by the Palm-Weevil, with abstract of evidence given by Planters, Belize, British Honduras. (Jan. 18, 1889.)

Prestoe, Henry. Report on Cocoa-nut Diseases, Demerera Times (1876, March 16).

Ridlex, H. N. Report on Destruction of Coconut Palms by Beetles, Singapore $(1889)$.

Riley, C. V., \& Howard, L. O. An Enemy to the Date Palm in Florida, Insect Life (1888), I, 14.

Bark Lice on the Cocoa-nut, Ibid (1889) I, 355.

Notes on the Palm Weevil, Ibid (1891), IV, 136.

The Coconut and Guava Mealy-wing, Ibid (1893), V, 5, 314.

Russell, W. The Cocoanut Palm: Its Culture and Diseases (1876).

Short, John. A Monograph of the Cocoanut Palm or Cocos nucifera, illustrated (1888).

Signonet, V. Essai sur les Cochenilles, Ann. Soc. Ent. de France (1869) (4), IX, 120 and 121, Pl. XII, figs. 8 and $8 \mathrm{~A}$.

Simon, M. F. Report on Beetles Injurious to Coconut Trees, Straits Settlements Government Gazette (1887, May 27). Tropical Agriculturist (1887), VII, 548.

Stanton, W. A., S. J., Notes on Insects affecting the Crops in the Philippines. Some Insect Enemies of the Cocoanut Palm. Bul. Phil. Weather Bureau (August, 1903), 223.

Summers, S. V. Notes on Rhynchophorus zimmermanni Sch. Can. Ent. (1873), $\mathrm{V}, 123$.

UrICH, F. W. Notes on Some Insect Pests of Trinidad, B. W. Ind. Insect Life (1894), VI, 196 et seq.

Vermont, J. M. B., and Kennedy, J. V. Report by the Select Committee of the Legislative Council on the Coconut Trees Preservation Bill. Penang, Straits Settlements (Dec. 23, 1889).

"W. B. L." Coconut Cultivation. Tropical Agriculturist (1884-5), IV, 1-3, $38-40,375-376$. 
$\cdot$ 


\section{ILLUSTRATIONS.}

[All figures are more or less magnified; exact size is indicated in descriptions.]

\section{Plate I.}

('oconut leaf showing work of Padraona chrysozona Plötz. Note that on some of the leaflets the apical portion of the blade has been eaten, while in others the basal portion is represented by the midrib alone.

Plate II. Drawn by W. Schultze.

Fig. 1. Coconut leaflet showing abandoned notches made by young larva of Padraona chrysozona Plötz.

2. Egg on margin of leaf.

2 A. Profile of egg.

3. Full-grown larva.

3 A. Profile of head.

4. Pupa.

4 A. Lateral view of pupa.

4 B. First thoracic spiracle or stigma.

Plate III. Drawn by W. Schultze.

Fig. $\quad$ 1. Padraona chrysozona Plötz, male.

2. Female.

Plate IV.

Fig. $\quad$ 1. Chalcis obscurata Walk., adult.

2. Coconut leaflet with cocoons of Braconid parasitic on P. chyrsozona Plötz together with shriveled caterpillar skin of latter.

\section{Plate V.}

FIG. 1. Thosea cinereamarginata Banks, full-grown larva.

2. Pupa.

2 A. Lateral view of pupa.

\section{Plate VI.}

Figs. 1, 1 A. Coconut leaflet with adult females and young of Aspidiotus destructor Sign. Note arrangement of young along veins.

2. Coconut leaflet attacked by disease causing spots similar to those produced by $A$. destructor Sign.

\section{Plate VII.}

Fig. $\quad$ 1. Aspidiotus destructor Sign., adult male.

2. Female puparium, showing adult and eggs.

3. Male puparium.

4. Egg.

5. Antenna of larva, distal segment.

6. Pygidium of female.

7. Antenna of adult male, distal segment.

8. Young larva. 
Plate VIII. Drawn by T. Espinosa.

FIG. $\quad$ 1. Scymnus sp., adult.

2. Larva.

3. Antenna of adult.

4. Maxillary and labial palpi and labium.

Plate IX.

Coconut leaflet showing male and female puparia of Chionaspis candida Banks. Note group of male scales around female at lower right. A few male and female scales of Chrysomphalus propsimus Banks occur also.

Plate X.

Chrysomphalus propsimus Banks. Male and female puparia on leaflet of coconut. 
Banks: The Principal ingects, etc.] [Phil. Journ. Scr., Vol. I, No. 3.

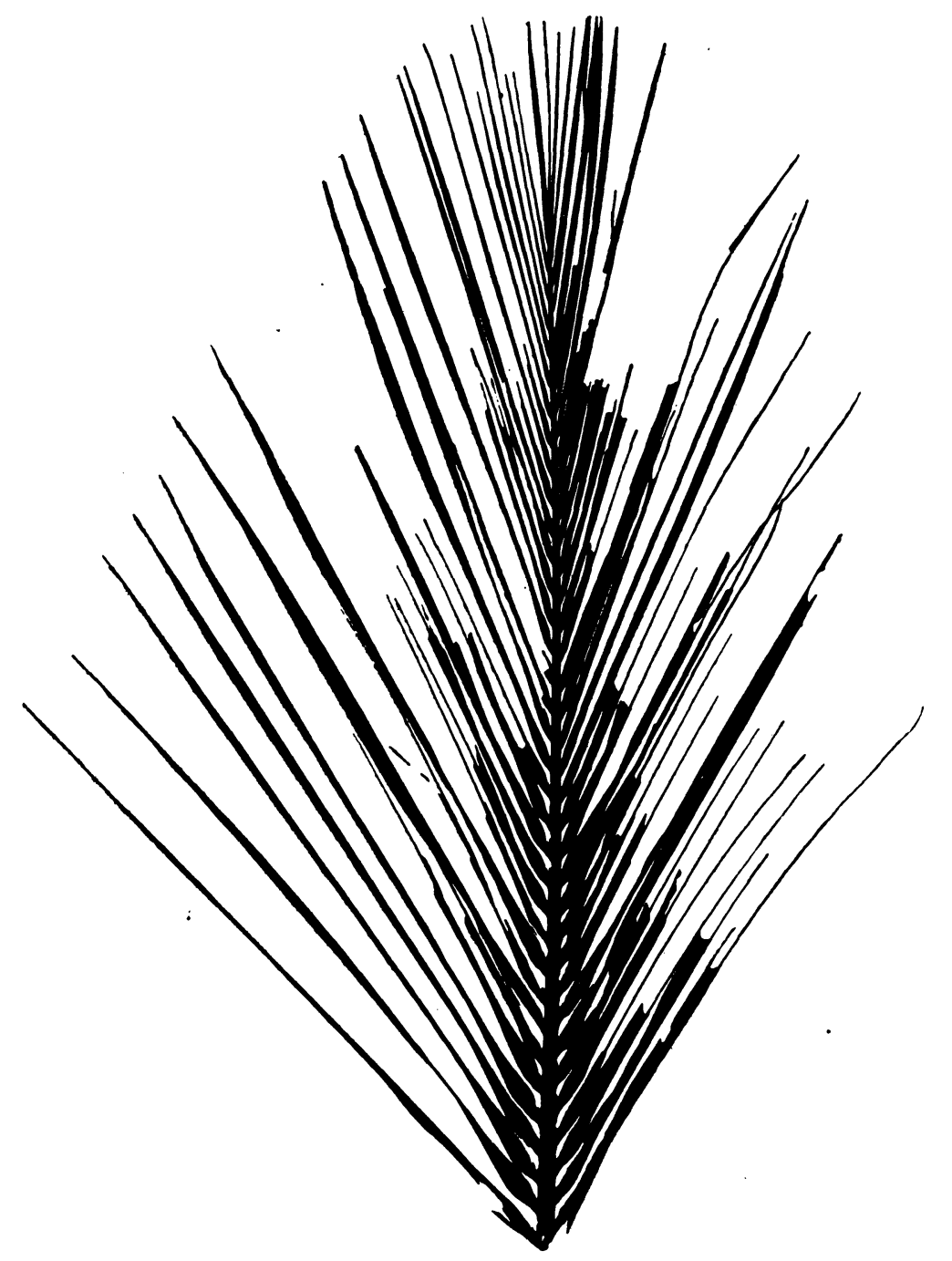

Piate I. 



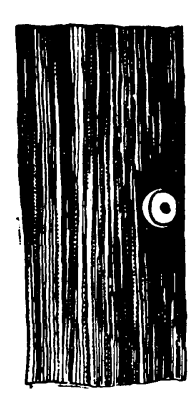

2

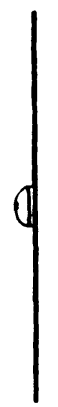

$2 \mathrm{~A}$
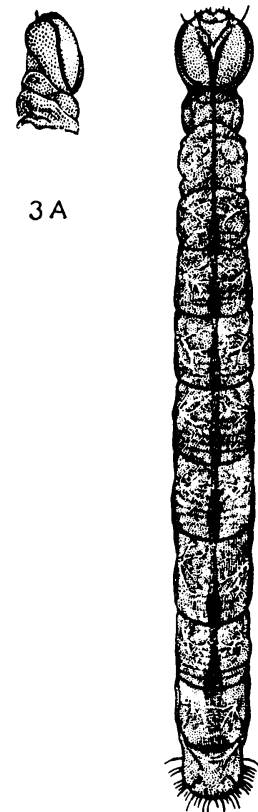

$3 \mathrm{~A}$

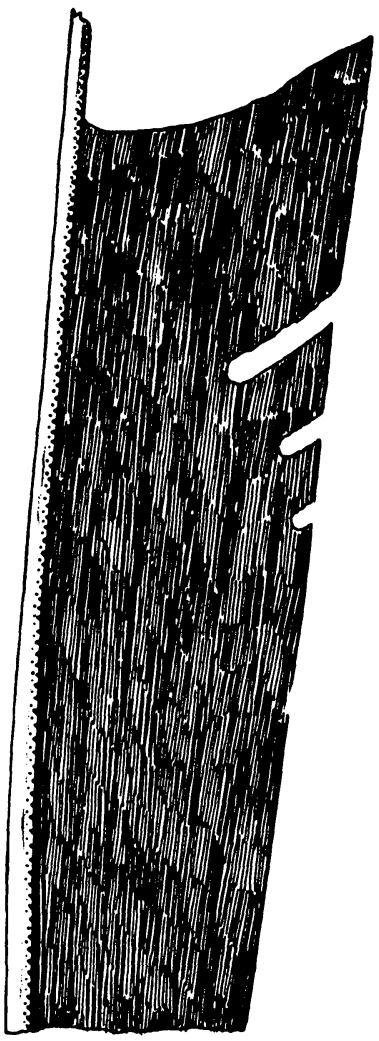

1

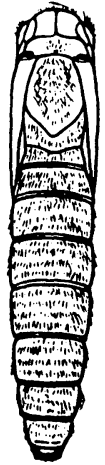

4

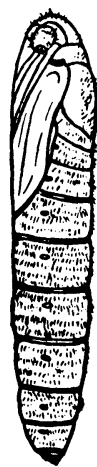

$4 \mathrm{~A}$

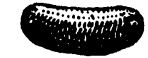

$4 \mathrm{~B}$

Plate II. 



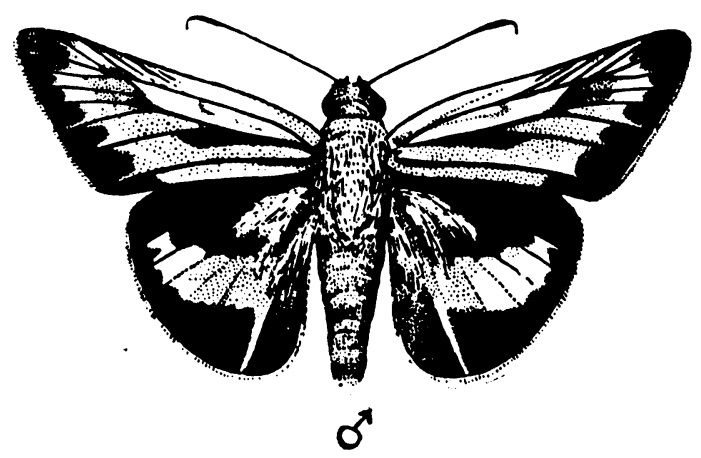

I

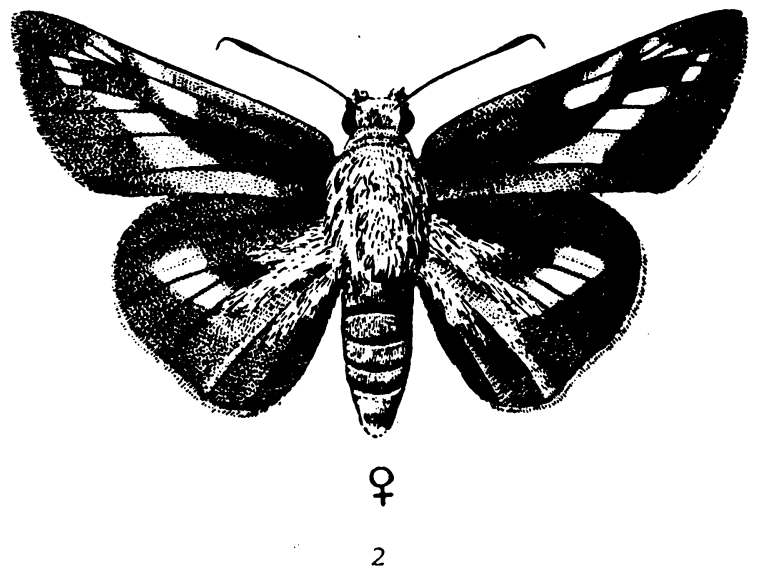

Plate III. 


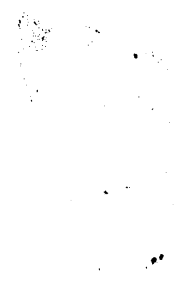




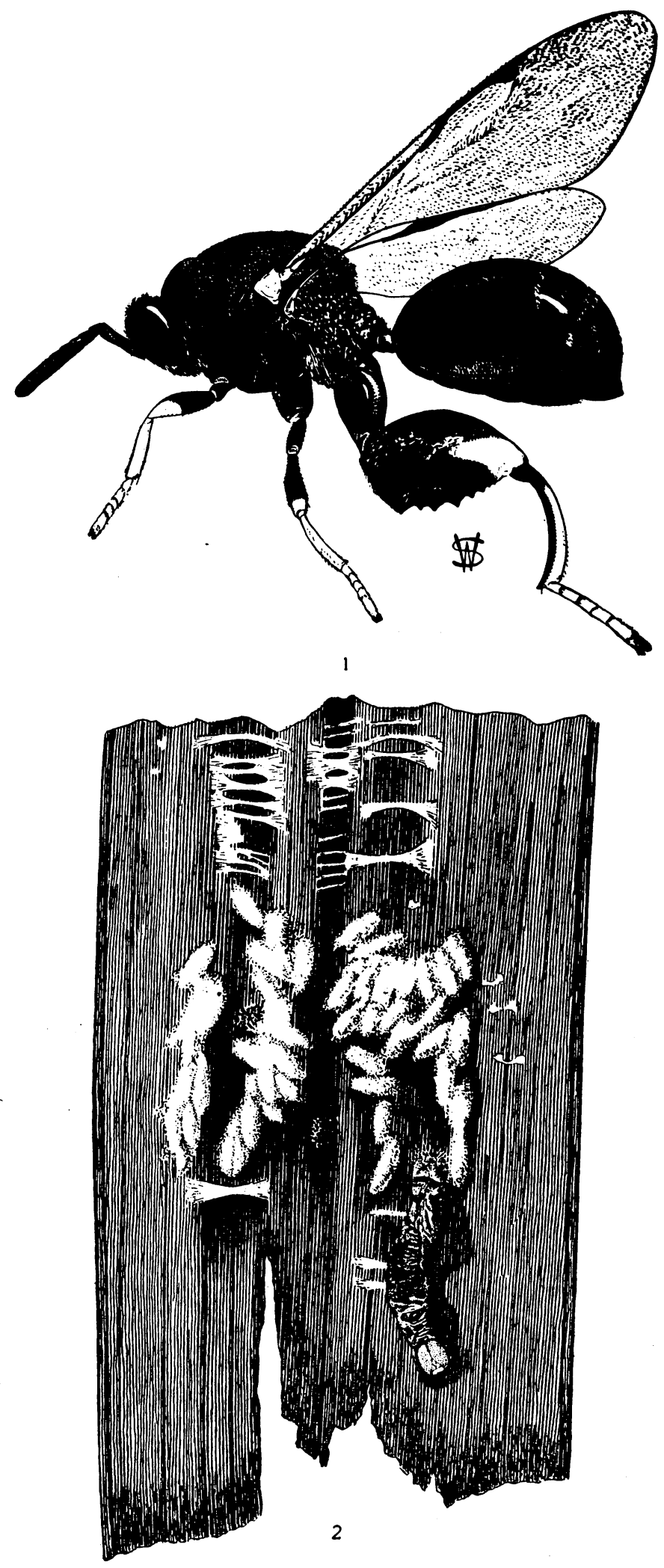

Plate IV. 
- 


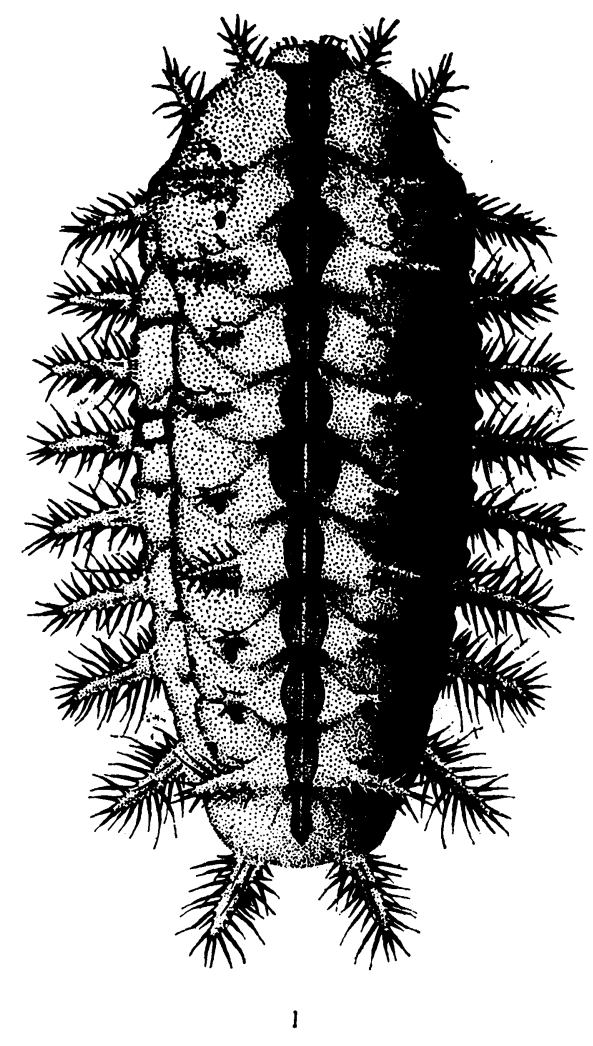

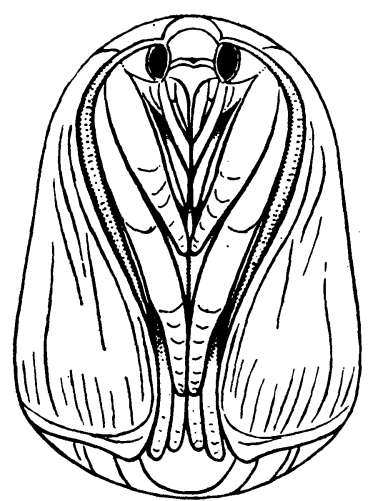

2

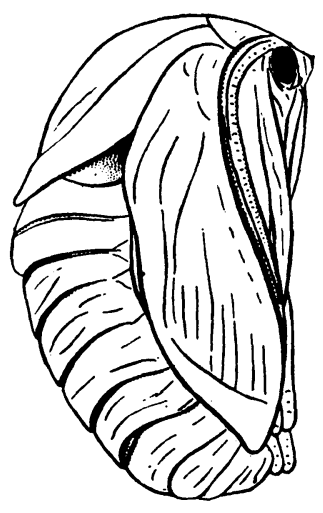

$2 \mathrm{~A}$

Plate V. 


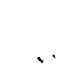




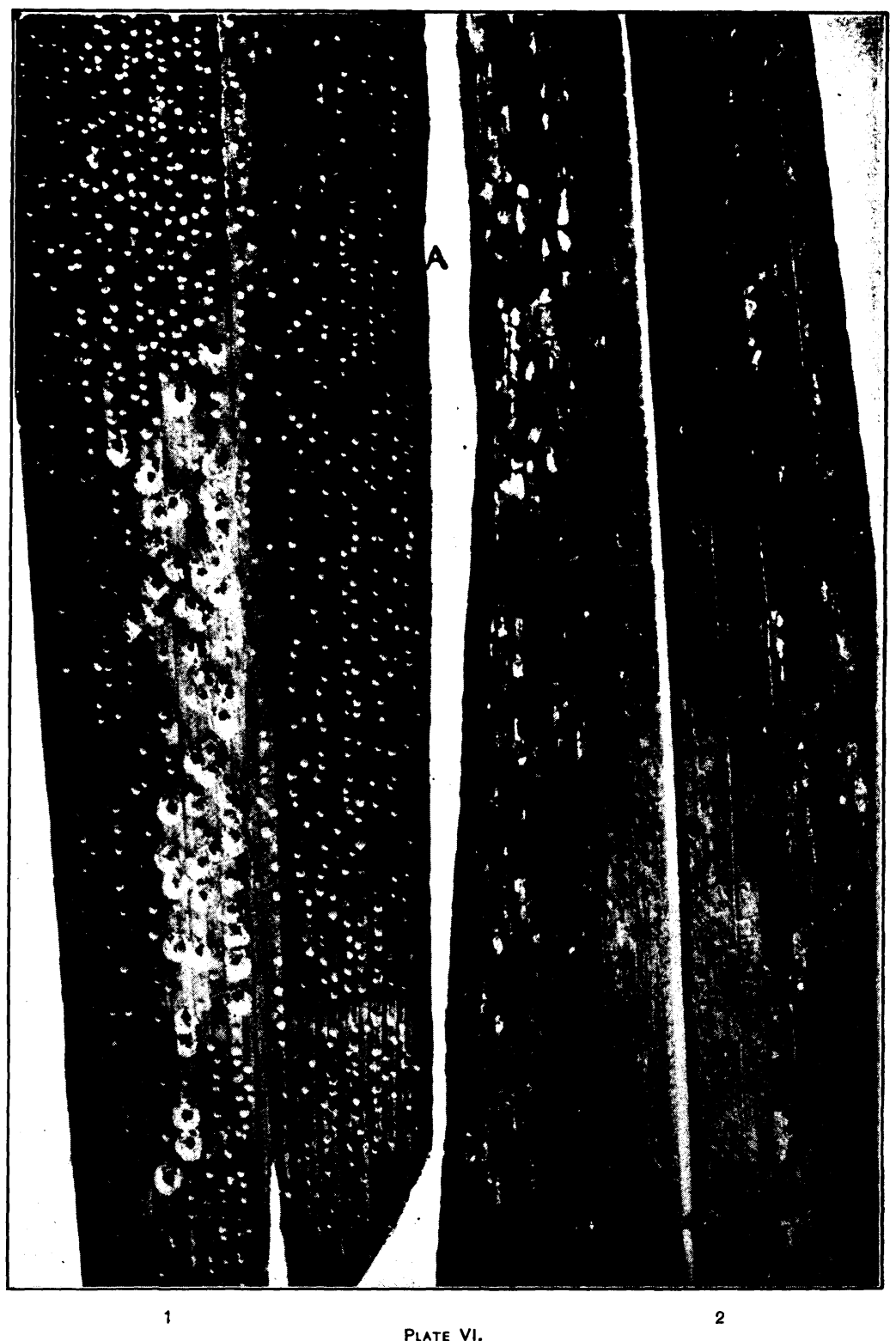


. 


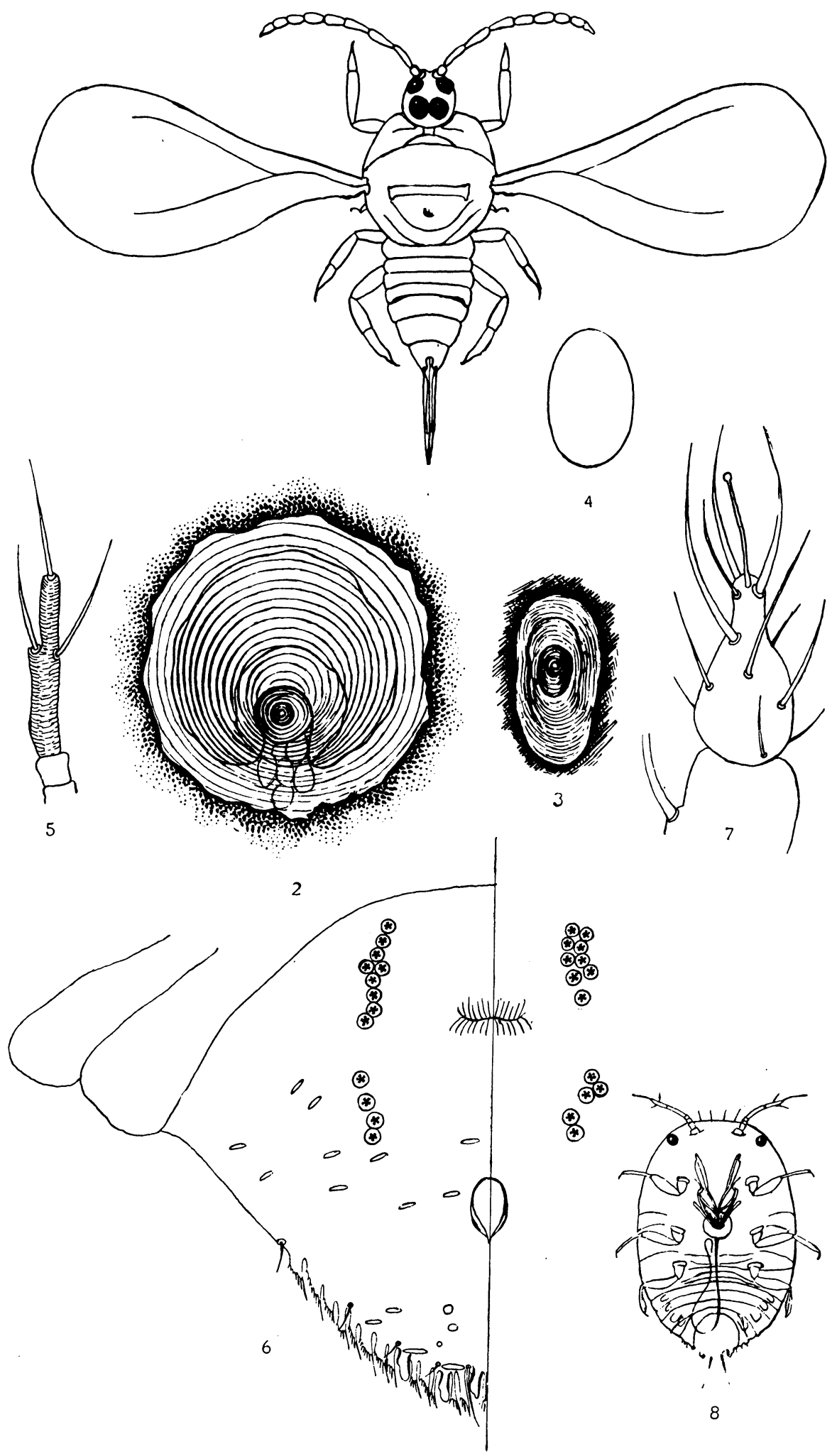

Piate Vil. 
. 

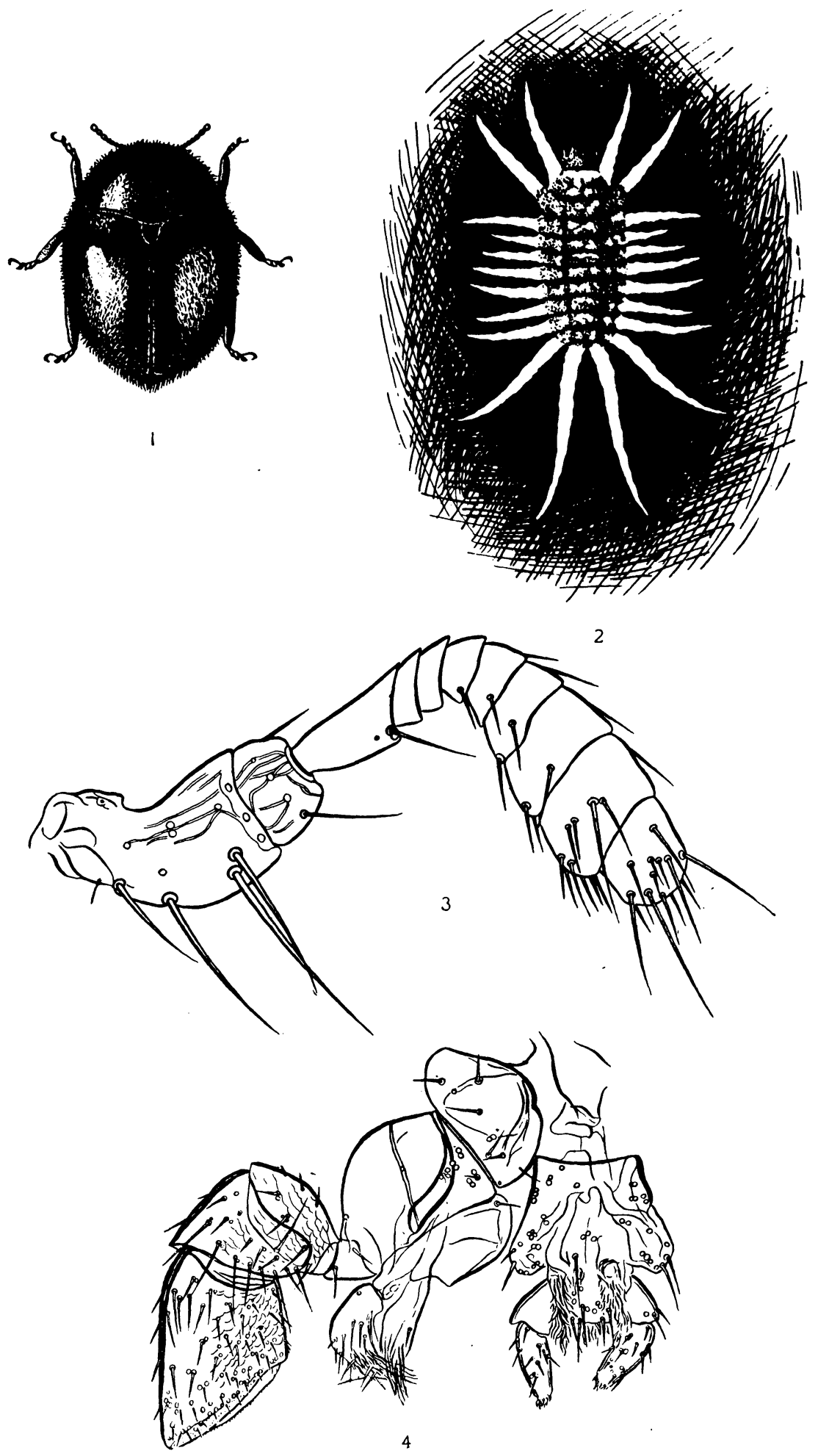

Plate Vili. 
$\therefore$

. 


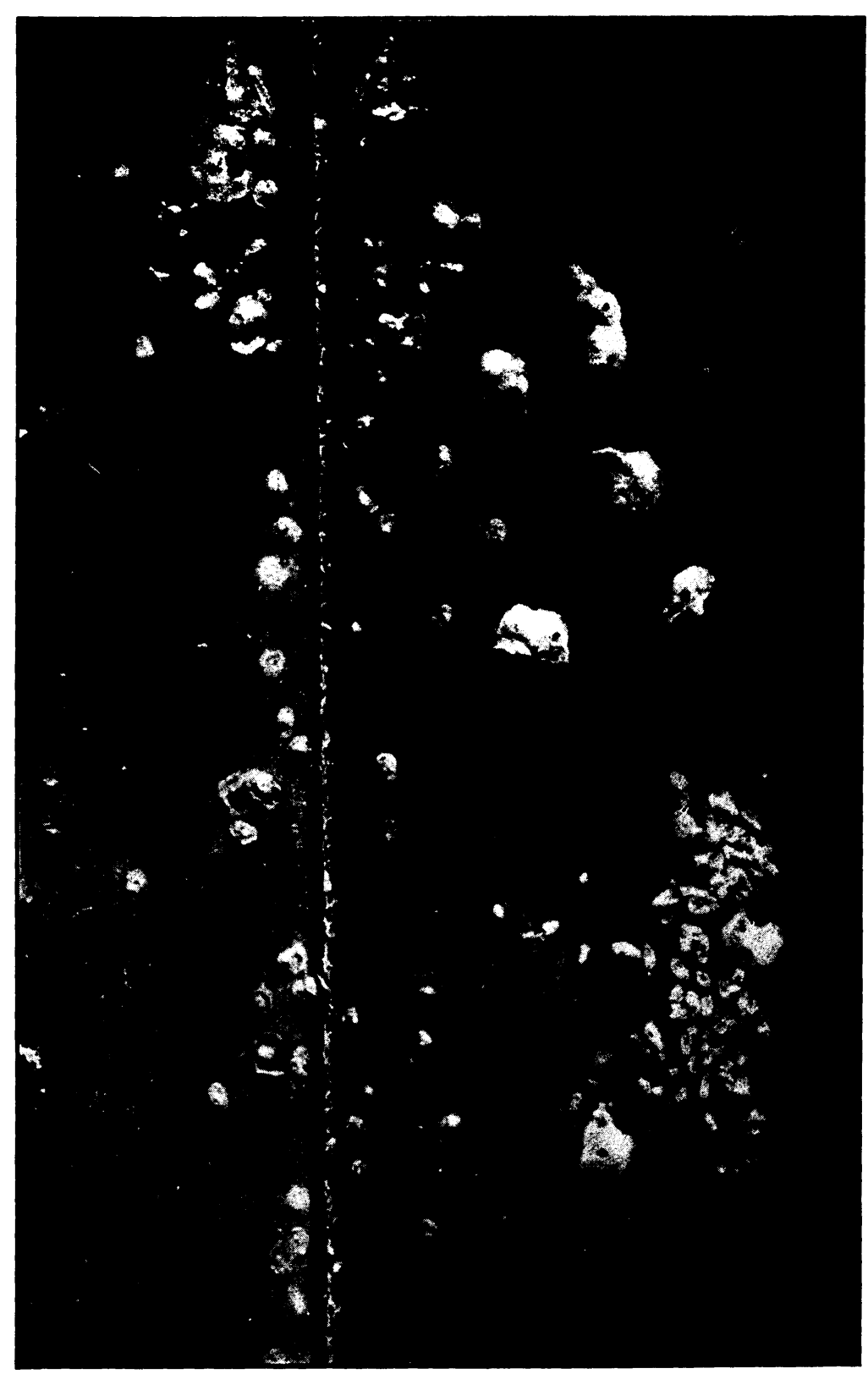

Plate iX. 



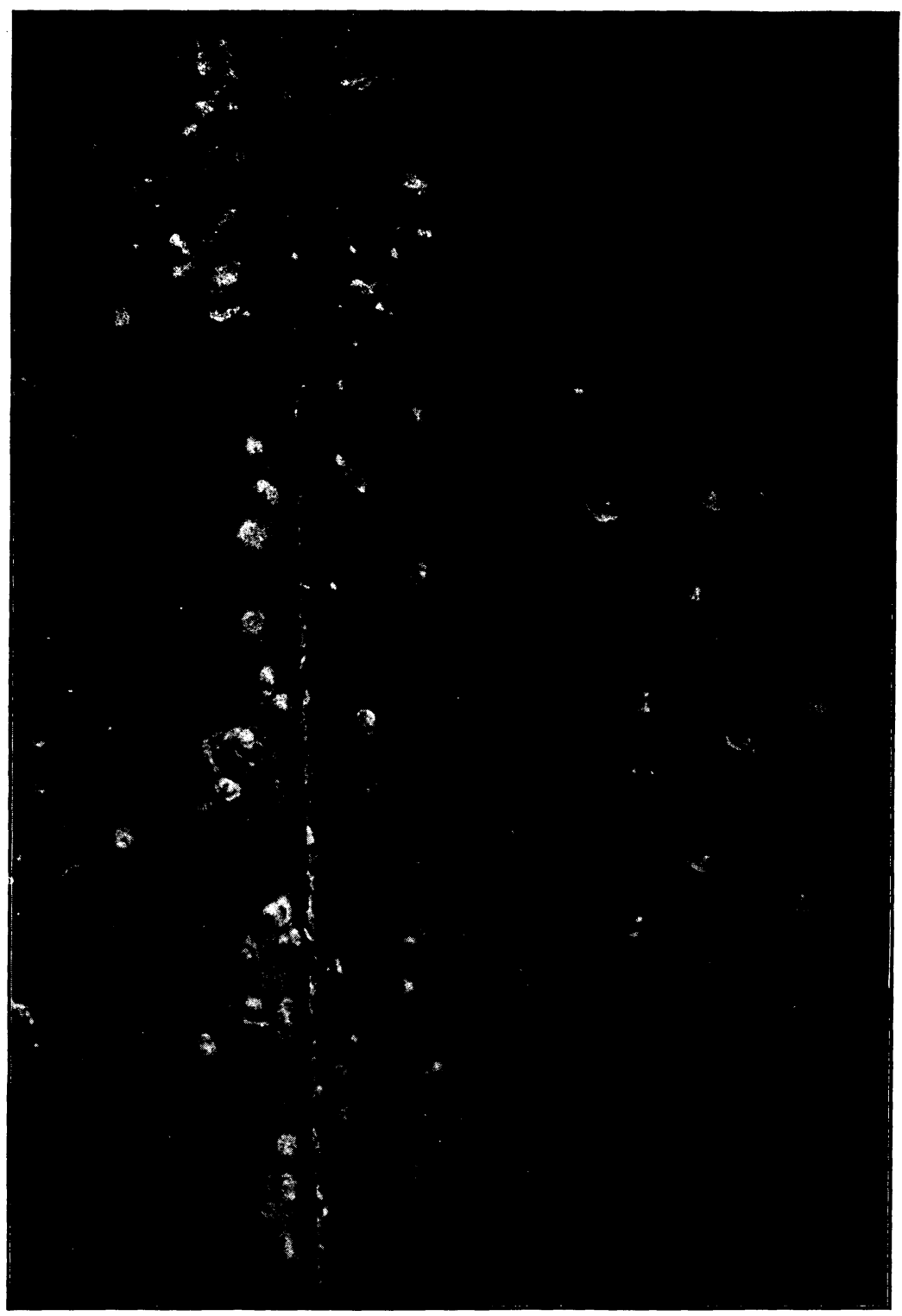

Plate $X$. 
$$
\text { - }
$$ 



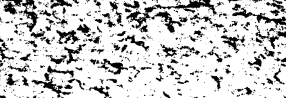
帮 8

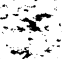

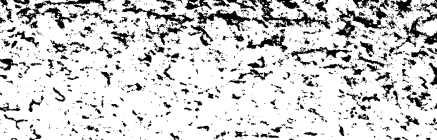

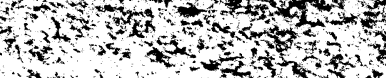

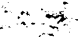
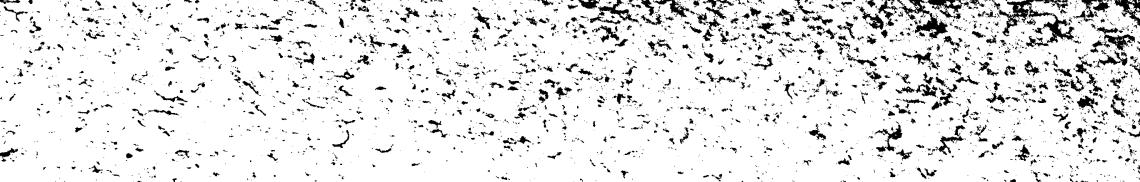



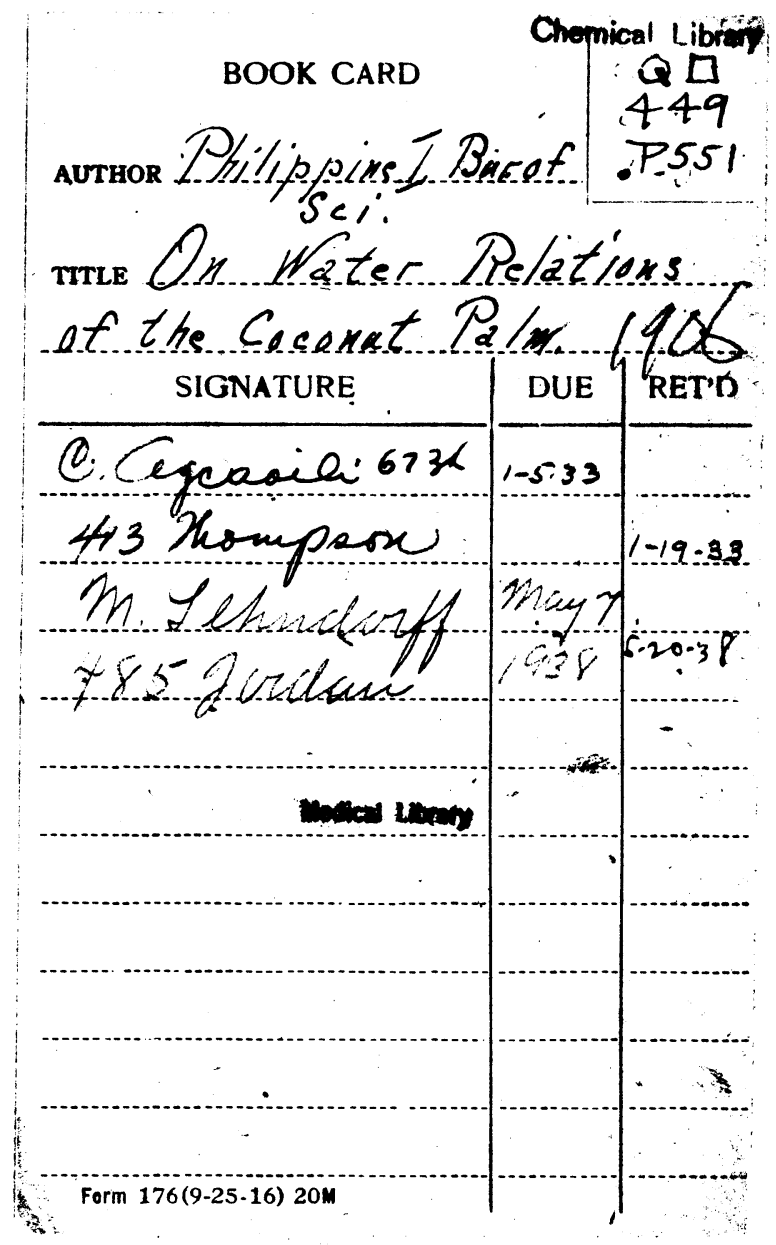


